\title{
Detailed analysis of public RNAseq data and long non-coding RNA: a proposed enhancement to mesenchymal stem cell characterisation
}

\author{
Sebastien Riquier ${ }^{1}$, Marc Mathieu ${ }^{1}$, Anthony Boureux ${ }^{1}$, Florence Ruffle $^{1}$, \\ Jean-Marc Lemaitre ${ }^{1}$, Farida Djouad ${ }^{1}$, Nicolas Gilbert ${ }^{1}$ and Therese Commes ${ }^{1}{ }^{*}$
}

February 27, 2020

\begin{abstract}
The development of RNA sequencing (RNAseq) and corresponding emergence of public datasets have created new avenues of transcriptional marker search. The long non-coding RNAs (lncRNAs) constitute an emerging class of transcripts with a potential for high tissue specificity and function. Using a dedicated bioinformatics pipeline, we propose to construct a cell-specific catalogue of unannotated lncRNAs and to identify the strongest cell markers. This pipeline uses $a b$ initio transcript identification, pseudoalignment and new methodologies such as a specific k-mer approach for naive quantification of expression in numerous RNAseq data.

For an application model, we focused on Mesenchymal Stem Cells (MSCs), a type of adult multipotent stem-cells of diverse tissue origins. Frequently used in clinics, these cells lack extensive characterisation. Our pipeline was able to highlight different lncRNAs with high specificity for MSCs. In silico methodologies for functional prediction demonstrated that each candidate represents one specific state of MSCs biology. Together, these results suggest an approach that can be employed to harness lncRNA as cell marker, showing different candidates as potential actors in MSCs biology, while suggesting promising directions for future experimental investigations.
\end{abstract}

\section{Introduction}

1

The increasing popularity of RNAseq and the ensuing aggregation of this data-type into public databases enable the search for new biomarkers across large cohorts of donors or cell types for the purpose pathological conditions or cellular lineages identification. As such, RNAseq has paved the way for the discovery of novel transcriptional biomarkers such as long noncoding RNAs (lncRNAs), that have emerged as a fundamental molecular class. A growing number of lncRNAs have been identified in the last decades, with their number approaching that of coding RNAs (17910 annotated human lncRNAs in the latest v32 version of the GENCODE versus 19965 coding genes).

*To whom correspondence should be addressed: Tel: +330467335711 ; Email: therese.commes@inserm.fr

${ }^{1}$ IRMB, University of Montpellier, INSERM, Montpellier, France. 
An increasing body of evidence has highlighted characteristics that define lncRNAs as therapeutic targets as well as potential tissue-specific markers [1]. Indeed, despite their non-coding nature, a large spectrum of functional mechanisms have been associated to lncRNAs [2, 3]. These include: endogenous competition (miRNA sponging for example), protein complex scaffolding and guides for active proteins with RNA-DNA homology interactions. These mechanisms occur in various physiological or pathological processes such as development, cancer and immunity [4, 5, 6]. To date, there is no finite list of long non-coding isoforms, making it difficult to construct a complete lncRNA catalogue due to the high number of transcripts and their tissue-specific expression $[7,8]$. The absence of a complete catalogue makes it difficult to establish a comprehensive lncRNA expression profile.Thus, currently, the best strategy for the study of lncRNAs consists in the prediction of transcripts from a selection of RNAseq data in a tissue-specific condition. This strategy was successful in novel lncRNA biomarker discovery in pathological conditions [9, 10], but was poorly explored for cell lineage characterisation. Taking into account their functional importance and specificity, these RNAs should therefore not be ignored in establishing the molecular identity of a cell type.

Cell characterisation by specific markers bring different challenges such as the importance of probing the specificity of the marker and its limits in an extended number of various cell types, rather than using a control/patient experimental model. Moreover, the cells are not in a fixed state and display a variable transcriptional activity depending on cell status, environment, culture conditions and other parameters [1]. Furthermore, the lncRNAs' function is generally poorly assessed, except in the case of recurrent known transcripts (HOTAIR, H19). Thus, the in silico elaboration of a lncRNA catalogue that document the functional domains where the candidates could act, will be beneficial in the identification of lncRNAs' role and thus, in future experiments.

To this end, we have developed an integrated four-step procedure consisting of: i) an $a b$ initio transcript reconstruction from RNAseq data and characterisation of novel transcripts, ii) a differential analysis using pseudoalignment coupled with a machine learning solution in order to extract the most cell-specific candidates, iii) an original step of tissue-expression validation with specific k-mers search in large and diversified transcriptomic datasets iv) an in-depth analysis to predict lncRNAs' functional potential from in silico prediction approaches. The notable advantage of introducing an in silico verification using k-mers is to allow a precise and in-depth determination of lncRNAs expression profile and to quickly interrogate their lineage specificity. In addition to that, validation of newly identified lncRNAs has been undertaken using RT-qPCR and long read sequencing (with Oxford Nanopore technology).

Mesenchymal Stem Cells (MSCs) are defined as multipotent adult stem cells, harvested from various tissues, including Bone Marrow (BM), Umbilical Cord (UC), and Adipose tissue (Ad). MSCs are an interesting cell type to explore since these cells lack the extended transcriptional characterisation that could highlight their lineage belonging and/or the possibility of distinguishing them from other mesodermal cell types such as fibroblasts and pericytes [11, 12]. The commonly admitted surface markers for MSCs, proposed by the International Society for Cellular Therapy (ISCT), and required to identify MSCs since 2006 are THY1 (CD90), NT5E (CD73), ENG (CD105) concerning the positive markers, and CD45, CD34, CD14 or CD11b, CD79alpha or CD19 and HLA-DR concerning the negative markers [13]. These markers are not distinctive and 
may therefore not be sufficient for the definition of cellular or biological properties. Considering their different therapeutic properties (chondro and osteo differentiation potential, immunomodulation and production of trophic factors) [14] and given the increasing usage of these cells for academic and preclinical research [15], a detailed molecular characterisation of MSCs and predictive marker of functionality will constitute an important tool in regenerative medicine. lncRNAs have emerged as a class of transcripts with tissue-specific expression and important functions, such as the regulation of MSCs function [16, 17, 18], and remain largely unexplored in these cells.

To address this need, we performed a broad transcriptomic analysis of novel lncRNAs on human Mesenchymal Stem Cells (MSCs). We started from publicly available MSCs RNAseq, selecting ribodepleted datasets in order to enhance lncRNAs discovery and to explore the poly-(A)+ as poly(A)- lncRNAs. We restricted the differential expression analysis to a bone-marrow MSCs source compared to "nonMSC" cell counterparts. Once achieved, in depth in silico analysis was performed to check the lncRNA cell specific profiles with more and extensive datasets. To validate our approach, RNAseq data from eight publicly available libraries of normal MSCs containing a large diversity of noncancerous cell types were used for novel lncRNAs detection and tissue expression comparison. We initially reconstructed more than 70000 unannotated lncRNAs present in human bone-marrow MSCs. These lncRNAs were assigned depending on their position relative to annotated genes: MSC-related Long Intergenic Non Coding trancripts" named "Mlinc", and MSC-related Long Overlapping Antisense transcripts called "Mloanc". Among them, 35 Mlincs were specifically enriched in the cell lineage compared to the "non-MSC" counterpart group. Finally, after a further selection of the three most specific Mlincs, detailed in vitro and in silico functional exploration were performed.

\section{Material and methods}

\subsection{Data collection and basic processing}

The public RNAseq datasets (in fastq format) have been assessed using the ENCODE, EBI ArrayExpress service or SRA database at each step of the pipeline:

i) lncRNAs prediction and first differential analysis (Table S1), ii) k-mer search in ENCODE data to refine lncRNAs' specificity (Table S2), iii) k-mer search in FANTOM6 CAGE dataset and single cell analysis (scRNAseq) from Adipose MSCs by X. Liu et al raw data [19] for functional investigations (Table S3), iv) k-mer search in MSCs in different conditions (Table S4).

The reads quality were assessed with FastQC (https://www.bioinformatics.babraham.ac.uk /projects/fastqc/) to avoid the implementation of poor quality data in the analysis. Data from Peffers et al. [20], added to ENCODEs BM-MSCs RNAseq data, were selected for the MSClinc and Mloanc characterisation and the differential step analysis considering the above-mentioned features: Ribo-zero technology, stranded and paired-ends RNAseq. Peffers data had a forwardreverse library orientation instead of a reverse-forward orientation of a classic Illumina sequencing, thereby the order of paired files was manually reversed. The fastq files used for lncRNAs prediction, referred as "MSC" group, were used for the differential analysis against the other cell types as "non-MSC" group, (Table S1) were mapped using CRAC v2.5.0 software [21] on the indexed GRCh38 human genome, including mitochondria, with stranded, -k 22 and rf options. 


\section{$2.2 \quad$ Ab initio assembly for transcripts prediction or unannotated tran- scripts prediction}

The aligned reads of the "MSC" group were put through ab initio transcript assembly. Unannotated transcripts were predicted with the following procedure: i/an ab initio reconstruction was performed on individual RNAseq with the StringTie [22] version 1.3.3b, with -c 5 -j 5 rf -f 0.1 (5 spliced reads are necessary to predict a junction and 5 reads at minimum are required to predict an expressed locus), ii/ the output individual gtf files obtained with the RNAseq of "MSC" group were then merged with the StringTie version 1.3.3b with -f 0.01 -m 200 and with a minimum TPM of 0.5, with the Ensembl human annotation (hg38) v90 used as guide for StringTie. The GTF was parsed with BEDTools [23] to dissociate new intergenic lncRNAs (lincRNAs) from annotated RNAs (coding or annotated lncRNAs), by applying filter criteria classically used in lncRNAs prediction [24], excluding transcript models overlapping (by 1 bp or more) any annotated coordinates. The resulting GTF of unannotated lincRNA from MSCs is referred as "Mlinc".

In parallel, the GTF was parsed with BEDTools to dissociate overlapping-antisens lncRNAs (named Mloanc), by applying filter criteria classically used in lncRNAs prediction, keeping transcript overlapping any annotated coordinates, then excluding transcript models overlapping these annotated coordinates on the same strand. The resulting GTF of MSCs overlapping-antisens lncRNAs is referred as "Mloanc" (Figure 1).

\subsection{Long-read sequencing}

The library was generated with $250 \mathrm{ng}$ polyA + mRNA purified from $50 \mu \mathrm{g}$ of human BM-MSCs total RNA. The polyA + mRNA were treated according to the cDNA-PCR sequencing kit protocol (ref SQK-PCS108) as recommended by Oxford Nanopore. 3254396 sequences were obtained on the Oxford Nanopore Minion sequencer. The base calling was done with albacore version 2.2.7. 2 720928 long-reads were successfully mapped using Minimap2 [25] version 2.10-r764 on GRCh38 human genome with default options used for Oxford Nanopore technology.

\subsection{Quantification with pseudoalignment and feature selection}

Kallisto v0.43.1 [26] was used directly on RNAseq raw fastq from the "MSC" and "non-MSC" groups. This pseudoalignment was performed with a number of bootstraps (-b) of 100, using a Kallisto index containing the sequences of all transcripts: the Ensembl coding and non-coding transcripts (v90) plus the predicted lincRNA and lncoaRNAs. Sleuth version 0.29.0 [27] was used with $\mathrm{R}$ for differential expression statistical analysis using the Walt test method, to compare the "MSC" group against the "non-MSC" group (including Lymphocytes, Macrophages, Hepatocytes, IPS, ESCs, HUVECs, Neurons, chondrocytes). Analysis was performed at the gene level for the annotated genes and at the transcript level for the predicted lincRNA and lncoaRNAs. Genes or lncRNAs having a log2 fold-change between "MSC" and others greater than 0.5 and a p-value lower than or equal to 0.05 were selected. Finally, only transcripts/genes overexpressed in MSCs were selected. Each category (annotated transcripts, lincRNAs and lncoaRNAs) of potential candidates passing the first differentiation expression filter were separated for feature selection analysis. Boruta 6.0 [28] was used with 10000 maximum runs and a pvalue of 0.01 on each category, with multiple comparisons adjustment using the Bonferroni method (mcAdj $=$ TRUE). Candidates passing the boruta test as "Confirmed" for each category were selected as reliable biomarkers. 


\subsection{Quantification by k-mers search}

To quantify the expression of a transcript or a gene in available RNAseq with a rapid procedure, specific k-mers of 31 nt length were extracted from the candidate sequence. A specific k-mer of an annotated candidate corresponds to a 31nt sequence that maps once on the genome and once on the reference transcriptome (Ensembl v90). In case of unannotated transcript (Mlinc, Mloanc) a specific k-mer maps once on the genome and is absent from reference transcriptome. The automated selection of specific k-mers is ensured by the Kmerator tool (in preparation) (https://github.com/Transipedia/kmerator). The k-mers were then quantified directly in raw fastq files using countTags (https://github.com/Transipedia/countTags). The quantification is expressed by the average count of all k-mers for one transcript, normalised by million of total $\mathrm{k}$-mers in the raw file.

In FANTOM6 Dataset (https://doi.org/10.1101/700864, article not peer-reviewed) containing CAGE Analysis, to approach a "Transcript Per Millions" normalisation, the number of k-mers quantified was normalised by the total number of reads in million.

\subsection{Genomic intervals assessment}

DNase-seq intervals of enrichment were directly downloaded from ENCODE in bed format for BM-mesenchymal cells (ENCFF832FHZ) and hematopoietic progenitors (ENCFF378FCS). The H3K27ac (GSM3564514) and H3K4me3 (GSM3564510) ChIP results from undifferentiated BMMSCs of the Agrawal Singh S. et al. study [29] were downloaded from GEO database in wig format, and remapped to the hg38 genome with CrossMap (http://crossmap.sourceforge.net/).

\section{7 in silico functional prediction}

We used LncADeep [30] to identify particular correlations between candidates and proteins. Beginning with our selection of 3 candidates, we filtered shared predicted proteins and selected protein uniquely predicted as interacting uniquely with the concerned candidate. The pathways concerned with these unique protein were identified with reactome.

Tarpmir was used to identify possible target site of human miRNA from miRbase $(\mathrm{p}=0.5)$ [31] and FEELnc [32] to decipher the coding potential of candidates, using the coding and non-coding par of Ensembl annotation sequences as model.

\subsection{Single-cell analysis}

Single-cell data were pseudoaligned with Kallisto, with the same index used for the initial bulk RNAseq analysis. Pseudoalignment of 10X genomics data, correction, sorting and counting was made by Kallisto "bus" functions. Count matrices were processed with Seurat R package [33, 34]. Empty droplets were estimated by barcode ranking knee and inflection points, only droplet with a minimal count of 10000 were kept. In the end, 26071 droplets remain.

After normalisation, Inter-donor batch effect was corrected with ComBat method in sva $\mathrm{R}$ package [35] (Combat function, prior.plots=FALSE, par.prior=TRUE). Cell cycle scoring was made by CellCycleScoring Seurat function, using gene set used by the initial authors [19]. Finally, other sources of unnecessary variability as percent of mitochondrial genes, cell cycle and number of UMIs were regressed using ScaleData Seurat function. 
To decipher genes enriched in cells positive for our markers, cells with a scaled expression superior or equal to 0.1 were labelled as positives, whereas cells with an expression inferior to the level were labelled as negatives. Then, markers of these cell were deciphered using FindAllMarkers Seurat function with a minimum FC threshold of 0.15. Expression of our markers in the Ad-MSCs population was made by FeaturePlot Seurat function after UMAP dimensional reduction, the gene enrichments were represented with VlnPlot function.

\subsection{Data visualisation}

Genome browser-like figures were generated with Gviz R package [36]. Bam tracks were generated from merged BAMs used for transcript prediction. Heatmaps were generated using superHeat $\mathrm{R}$ package (https://github.com/rlbarter/superheat).

\subsection{Ethics approval and consents}

Human primary MSCs was obtained from patients who had granted the authors written informed consent with approval of the General Direction for Research and Innovation, the department in responsible for questions of ethics within the French Ministry of Higher Education and Research (registration number: DC-2009-1052). Human primary myoblasts were collected from patients of the CHU of Montpellier, France (the Montpellier University Hospital) who had provided informed consent. All experiments were performed in accordance with the Declaration of Helsinki and approved by the ethical committee of the CHU of Montpellier (France). Samples were approved for storage by the French "Ministre de l'Enseignement et de la Recherche" (NDC-2008-594). Liver samples were obtained from the Biological Resource Center of Montpellier CHU (CRB-CHUM; http://www.chu-montpellier.fr; Biobank ID: BB-0033-00031). The procedure was approved by the French Ethics Committee and written or oral consent was obtained from the patients.

\subsection{Cell preparation and culture conditions}

MSCs were isolated from bone marrow aspirates of patients undergoing hip replacement surgery, as previously described [37]. Cell suspensions were plated in $\alpha$-MEM supplemented with $10 \%$ FCS, $1 \mathrm{ng} / \mathrm{mL}$ FGF2 (R\&D Systems), $2 \mathrm{mM}$ L-glutamine, $100 \mathrm{U} / \mathrm{mL}$ penicillin and $100 \mu \mathrm{g} / \mathrm{mL}$ streptomycin. These were shown to be positive for CD44, CD73, CD90 and CD105 and negative for CD14, CD34 and CD45 and used at the third or fourth passage. Human skin fibroblasts were cultured in DMEM high glucose supplemented with $10 \%$ FCS. For Ad-MSCs isolation, adipose tissue was digested with $250 \mathrm{U} / \mathrm{mL}$ collagenase type II for $1 \mathrm{~h}$ at $37{ }^{\circ} \mathrm{C}$ and centrifuged $(300 \mathrm{~g}$ for $10 \mathrm{~min}$ ) using routine laboratory practices. The stroma vascular fraction was collected and cells filtered successively through a $100 \mu \mathrm{m}, 70 \mu \mathrm{m}$ and $40 \mu \mathrm{m}$ porous membrane (Cell Strainer, BD-Biosciences, Le-Pont-de-Claix, France). Single cells were seeded at the initial density of 4000 cell/cm2 in $\alpha$ MEM supplemented with $100 \mathrm{U} / \mathrm{mL}$ penicillin/streptomycin (PS), $2 \mathrm{mmol} / \mathrm{mL}$ glutamine (Glu) and 10\% fetal calf serum (FCS). After $24 \mathrm{~h}$, cultures were washed twice with PBS. After 1 week, cells were trypsinised and expanded at 2000 cells/cm2 till day 14 (end of passage 1), where Ad-MSCs preparations were used.

Human umbilical vein endothelial cells (HUVEC) obtained from Clonetics (Lonza, Levallois Perret, France) were cultured in complete EGM-2MV (Lonza) supplemented with 3 \% FCS (HyClone; Perbio Science, Brebires, France) 
Primary human myoblasts were isolated and purified from skeletal muscles of donors, as described by Kitzmann et al [38]. Purified myoblasts were plated in Petri dishes and cultured in growth medium containing Dulbecco's Modified Eagle's Medium (Gibco) supplemented with $20 \%$ foetal bovine serum (FBS) (GE Healthcare, PAA), $0.5 \%$ Ultroser G serum substitute (PALL life sciences) and $50 \mu \mathrm{g} / \mathrm{ml}$ Gentamicin (Thermo Scientific, France) at $37^{\circ} \mathrm{C}$ in humidified atmosphere with $5 \% \mathrm{CO}_{2}$. All experiments were carried out between passage $4(\mathrm{P} 4)$ and $\mathrm{P} 8$ to avoid cell senescence.

IPSCs were maintained in mTeSR-1TM medium (STEMCELL Technologies), in Petri dishes with matrigel (Corning, France). For the passages, cells were incubated in Gentle Cell Dissociation Reagent (STEMCELL Technologies) at room temperature, dissociation medium was discarded and cells incubated in mTeSR medium. All cell cultures were performed at $37^{\circ} \mathrm{C}$ with $5 \%$ of $\mathrm{O}_{2}$ and $10 \%$ of $\mathrm{CO}_{2}$.

Primary human hepatocytes (PHHs) were isolated, as described previously [39], from liver resections performed in adult patients.

NSC derived from H9 or directly bought (StemPro) have been cultivated on laminine with StemPro NSC SFM medium.

H9 embryonic stem cells were cultivated in ESICO medium in a coculture H9/MEF (Mouse Embryonic Fibroblasts) at $37^{\circ} \mathrm{C}$ with $5 \%$ of $\mathrm{O}_{2}$ and $5 \% \mathrm{CO}_{2}$.

\subsection{RNA preparation and reverse transcription}

Total RNA was isolated using TRIzol reagent (invitrogen) or RNeasy Mini Kit (Qiagen, France) according to the manufacturer protocol. RNA was quantified using a NanoDrop ND-1000 spectrophotometer (Thermo Fisher Scientific, France). RNA quality and quantity were further assessed using the 2100-Bioanalyzer (Agilent Technologies, Waldronn, Germany). Only preparations with RNA integrity number (RIN) values above 7 were considered. Reverse-transcription was performed either with random hexamers using the GeneAmp Gold RNA PCR Core kit (Applied Biosystems) or with oligo(dT) using SuperScriptTM First-Strand Synthesis System for RT-qPCR (invitrogen, France).

\subsection{Real-time quantitative PCR}

Primer pairs were designed with primer3 online software (http://bioinfo.ut.ee/primer3-0.4.0/) from the transcripts' sequences. Primer pairs with a perfect and unique match on the human genome were validated with ucsc blat software (https://genome.ucsc.edu). As a final verification, primers were visualised in parallel with the bam alignment using IGV (http://software.broadinstitute.org/softv to verify that the primers overlap zones with read coverage. If possible, primer-pairs were designed to span an intron when present in the genomic sequence. Primers were designed for a mean Tm of $60^{\circ} \mathrm{C}$. Quantitative PCR (qPCR) were performed using LightCycler 480 SYBR Green I Master mix and real-time PCR instrument (Roche). PCR conditions were $95{ }^{\circ} \mathrm{C}$ for 5 min followed by 45 cycles of $15 \mathrm{~s}$ at $95{ }^{\circ} \mathrm{C}, 10 \mathrm{~s}$ at $60{ }^{\circ} \mathrm{C}$ and $20 \mathrm{~s}$ at $72^{\circ} \mathrm{C}$. For each reaction, a single amplicon with the expected melting temperature was obtained.

The gene encoding ribosomal protein S9 (RPS9) was used as house-keeping gene for normalisation. The threshold cycle $(\mathrm{Ct})$ of each amplification curve was calculated by Roche's LightCycler 480 software using the second derivative maximum method. The relative amount of transcripts were calculated using the ddCt method [40]. 


\section{Results}

For the purpose of generating a catalogue of all transcripts in any particular cell type, we developed a pipeline for the characterisation of all RNAs and their expression profile in a large collection of RNAseq data. The procedure includes four steps: i) an ab initio transcripts reconstruction from RNAseq data and identification of unannotated transcripts, ii) a differential analysis using pseudoalignment coupled with a machine learning solution in order to extract the most cell-specific candidates, iii) an original step of tissue- expression validation with a kmer approach (comparing large transcriptomic datasets), iv) an in-depth analysis to predict lncRNAs functional potential from in silico prediction approaches (Figure 1). To illustrate the procedure, we produced a RNA catalogue from bone-marrow MSCs ("MSC" group).

\subsection{General features of the predicted MSC catalogue of lncRNAs}

As mentioned above, we started with the ab initio reconstruction of any transcript from bonemarrow RNAseq with Stringtie assembler after mapping the reads with the CRAC software (see Materials and Methods for parameters). New isoforms of annotated transcripts were ignored. Of the 200243 transcripts present in Ensembl annotation (version 90), 105511 (52.6\%) were detected in MSCs ( TPM >0.1 in pseudoalignment quantification).

73463 new lncRNAs were reconstructed. This fraction of unannotated transcripts represent $41 \%$ of detected transcripts, so in our case the ab initio reconstruction made it possible to almost double the inventory of detectable signatures in MSCs (Figure 2A). Of these, 34712 were found to be intergenic and were thus referred to as "Mlinc" RNAs (MSC-related long intergenic non-coding RNAs), and 38751 were found to be overlapping coding regions but in anti-sense orientation and thus referred to as "Mloanc" RNAs (MSC-related long overlapping antisense non-coding RNAs, with criteria as described in materials and methods and Figure 2A).

The $a b$ initio method by itself is not sufficient to efficiently determine the lncRNAs' full length sequences. Moreover, this step does not preclude the possibility of false positives and at this point of the analysis, all the different rebuilt transcripts are considered to be windows of RNA expression or possible artefacts. These candidates are filtered and, for most interesting candidates, their true form is to be refined through experimental methods. We also assessed the general characteristics of predicted de novo lncRNAs in MSCs. Globally, Mlincs and Mloancs are shorter transcripts with longer exons compared to coding genes and annotated lncRNAs. The large majority of predicted lncRNAs are mono exonic ( $99 \%$ for Mlincs, $79 \%$ for Mloancs), with a length close to 200nt (Figure 2B-C). A consequence of the abundance of mono-exonic lncRNAs is an infinitesimally small number of variant forms. Only $0.15 \%$ and $0.82 \%$ of Mlincs and Mloancs are not mono isoforms, respectively.

The GC content of reconstructed lncRNAs is lower than that of coding or non-coding annotated genes (Figure 2D). This low GC proportion of around $40 \%$ is a common feature in ab initio transcript prediction, observed in a majority of studies of different species, from mammals, insects, plants or prokaryotes $[41,42,43,44]$.

\subsection{Enrichment of a restricted set of Mlincs and Mloancs}

In this second step, our objective was to obtain a restricted set of potential transcripts, using successive filtering approaches that would reveal their cell specificity. We quantified annotated transcripts, Mlincs and Mloancs with kallisto pseudoalignment [26] in a cohort constituted of two groups : the "MSC" group contained the BM-MSCs initially used for ab intio reconstruction, 
and the "non-MSC" group used for comparison, composed of a large panel of different cell types including hESC, hematopoietic precursors and stem cells, primary chondrocytes, IPS, hepatocytes, neurons, lymphocytes and macrophages (Table S1).

Only over-expressed transcripts in "MSC" group versus "nonMSC" group were selected. Differential statistical tests were made with Sleuth, a tool specially dedicated to Kallisto results [27] (see all selective parameters in Materials and Methods). We performed two differential expression analyses: one at the gene level for Ensembl annotation, and the other at the transcript level for unannotated transcripts, to give the most likely variant form of the predicted lncRNAs. After this differential analysis, 2801 annotated genes, 655 Mlincs and 3032 Mloancs are significantly overexpressed in BM-MSCs (Figure 2 E-F).

The lncRNAs are commonly known to be less expressed than coding genes and this was observed in our selected annotated genes and new lncRNAs (Figure 2G). As a validation of our procedure, we found the 3 positive MSCs markers of ISCT among the selected annotated genes: THY1 (CD90), ENG (CD105), and NT5E (CD73). We also retrieved some influencers of MSCs activity, for example WNT5A [45, 46], Lamin A/C [47], FAP [48] and others. The complete list of selected genes is provided in Table S5.

\subsection{Feature selection for the most discriminating coding and non- coding markers}

In an attempt to select the best candidates, we retained lncRNAs with the most discriminative profile between "MSC" and "non-MSC" groups. In our case, the limitation with a classical "top" ranking by Fold Change (FC) or p-value is the presence of subgroups of different types of cells inside the negative "non-MSC" group. The FC, estimated by the Beta value in Figure 2C, appears to be a biased indicator of differential expression as it can select strong but localised expressed lncRNAs in cells poorly represented in our negative group, leading to potential false positive results.

To avoid this problem, we used the Boruta feature selection (see material and method section), for selection of discriminating features based on "random forest" machine learning methodology. Boruta was used separately on each group of candidates (annotated genes, Mlincs and Mloancs) to extract a restricted representation of the most relevant MSCs signatures. The top 35 importance scores were selected for genes, Mlincs and Mloancs. We arbitrary chose to select the first 35 transcripts for each group based on our observation of a drop in the importance score in coding the gene series. Considering the expression profile of the top 35 coding genes and predicted Mlincs, BM-MSCs clusterised independently from other cells types (Figure 3A).

In contrast, the selection of Mloancs did not provide a satisfying clustering as they had similar expression profiles in MSCs and other closely related cell types, in particular in primary chondrocytes (Figure S1). For this reason, Mloancs were not retained for further analysis. Selected annotated genes showed a poor specificity, with only few candidates showing a clear difference of expression between MSC and others: APCDD1L, HOTAIR, KRTAP1-5 and SMILR. The 3 positive MSCs markers from the ISCT were absent in this selection. The novel top 35 Mlincs showed less expression overall but with a more distinctive profile and a higher number of possible MSCs markers with clear contrast of expression. The characteristics, genomic intervals and sequences of the 35 candidates are presented in Table S7.

To assess the potential of genes already proposed as potential MSCs biomarkers by ISCT (Figure S2) or other potential MSCs markers proposed by different authors [14] (Figure S3), we 
made a separated expression heatmap, without filter. Among these previously proposed markers, THY1 (CD90) presented the most specific profile. However, each gene presented expression in distinct non-MSC types.

\subsection{Validation of selected Mlincs with long reads sequencing}

As mentioned above, classical annotation of lncRNAs with ab initio short read methods suffers from inaccuracies and biases. The Oxford Nanopore long read technology (ONT) can sequence entire cDNA, which constitutes a clear technological advantage, not only in confirming the existence of the transcripts but also as it makes it possible to precisely identify the genomic intervals of lncRNA candidates. We performed long-read sequencing of a poly(A) RNA library obtained from a BM-MSCs sample. Among the top 35 selected Mlincs, with around 3 million total reads, 4 transcripts are covered with the ONT sequencing.

These intergenic lncRNAs are named as Stringtie output (SetName. TranscriptNumber. VariantNumber): Mlinc.28428.2, MlincV4.128022.2, MlincV4.89912.1 and MlincV4.64225.1. To support the above transcriptional units, we compared them with our short read data and searched for epigenetic status at the locus of the Mlincs in bone-marrow stromal mesenchymal cells. We looked at DNase sensitive site, H3K27 acetylation, H3K4 trimethylation that commonly corresponds to active regulatory regions (Figure 3B). We globally observed a DNA accessibility enrichment and acetylation of Histone 3 at the promotor region of our candidates, correlating with DNAse sensitivity hotspots in BM mesenchymal cells that reinforce the prediction of the expression windows. In particular, for Mlinc.28428.2, the transcript observed with long reads sequencing corresponded to the prediction made with short reads. It was also supported by Mlinc.28428.1, a variant that differ by the absence of the second exon. Similar characteristics were observed for Mlinc.128022, which also produced two variants with a different organisation of 5 exons. The two other candidates, Mlinc.89912.1 and Mlinc.64225.1 are mono-exonic. Mlinc.89912.1 occurs at the close proximity of FGF5 3' end, in reverse orientation at this locus. For this reason, the different epigenomic features could not be attributed with certainty to the Mlinc. For Mlinc.64225.1, the sequence is longer than the $a b$ initio short read prediction. KRTAP1-5, HOTAIR and SMILR, selected for their good expression profile, were also covered by long reads (Data not shown).

\subsection{High-throughput investigation of a marker's specificity by spe- cific k-mers search}

A marker can only be considered specific within the limits of the diversity of samples used for its study. Considering the growing number of cell/tissues and transcriptional profiles, it is essential to probe the limits of a chosen biomarker against these various cell types. Most of published analyses highlighting new potential biomarkers of MSCs or fibroblasts have been restricted to a comparison between only few cell types and, as discussed, commonly described markers are not strictly distinctive. In order to assess the expression of Mlincs candidates in a large number of samples, we extracted specific 31nt k-mers from each of their sequences, as previously described [49]. These simplified but candidate-specific (oligonucleotide-like) probes allow a simple and fast presence/absence search to be made on large-scale cohorts and a direct quantification in raw fastq data. The k-mers were quantified in ENCODE human RNAseq database, including "primary cells" and "in vitro differentiated cell" categories (Table S2). Particularly, as the bibliography suggests that MSCs can also express phenotypic characteristics of endothelial, neural, smooth 
muscle cells, skeletal myoblasts and cardiac myocytes, RNAseq samples from this mesodermal origin were tested.

With ISCT positive markers, we observed an expected expression profile that recapitulate previous biological studies, particularly the high expression of Endoglin (ENG, CD105) in endothelial cells (Figure S4) and the overexpression of NT5E (CD73) in epithelial and endothelial cells (Figure S5). Interestingly, their expression varied among MSCs cell sources : NT5E (CD73) was strongly enriched in adipose and BM derived MSCs, and THY1 (CD90) in umbilical cord derived MSCs (UC-MSCs) (Figure S6). We next analysed the expression profile using our candidate annotated genes Mlinc specific k-mers (Figure 4). The specific k-mers search supported the stated expression profile of Mlincs previously shown : our Mlinc candidates were positive in MSCs and displayed weak or absent expression in cells of ectodermal lineage, hematopoietic or endothelial origins.

However the high throughput and naive quantification in the ENCODE cohort made it possible to extend the observation of this absence of expression into cell types not previously studied. Moreover, this diversity showed that the expression of most of the candidates, contrarily to positive markers of the ISCT, were exclusive of cells with mesodermal origin. All candidates were expressed in at least one type of fibroblasts and differentially present in other mesodermal cell types. For the 4 selected Mlincs, they shared: (i) a systematic and strong expression in cell types like skin fibroblasts, and cells derived from reservoir of mesenchymal progenitors (muscle satellite cells or dermis papilla cells), (ii) a regular over-expression in regular cardiac myocytes, (iii) a significant and irregular expression in smooth muscle cells. The ENCODE cohort containing MSCs of different origins, we can therefore observe that the Mlincs show differences of expression depending of the tissular origin, these candidates being mainly expressed in two MSCs types. The results permitted the classification of our Mlincs according to observed specificity, from the most promising to the least restricted profile : Mlinc.28428.2 is expressed in Ad and BM derived MSCs. It is the candidate with the clearest absence of expression in non-mesodermal cells, and with the poorest relative expression in Smooth Muscle Cells (SMC). Mlinc.128022.2 is expressed in Adipose and Bone Marrow MSCs, and particularly in preadipocytes and muscle cells : (myoblasts, myocytes and myotubes). Mlinc.89912.1 is principally expressed BM-MSCs and less in UC-MSCs and ADMSCs, but shows expression in epithelial and endothelial cell. Finally, Mlinc.64225.1 differs from other Mlincs as it is also strongly expressed in keratinocytes, HSC, and epithelial cells (Figure S7). Its expression in critical non-MSC types has led us to retain the three other Mlincs for further investigations.

\subsection{RT-qPCR mimics the in silico prediction and deciphers multiple transcript variants}

To confirm the specificity of selected Mlincs' expression experimentally, we performed RT-qPCR on a set of 80 RNA preparations from different primary cell (Figure 4C). These includes MSCs from Bone Marrow (BM), Adipose (Ad) and Umbillical Cord (UC), fibroblasts of different tissue origins, IPS cells, neural stem cells, myoblasts, HUVECs and hepatocytes (Table S6). RT-qPCR and amplicon sequencing using sets of specific primers (Table S7) confirmed different predicted forms of the Mlincs candidates in BM-MSCs (Figure S8). We designed two primer pairs for both Mlinc. 128022 variants to validate the existence of first splice, and two pairs for Mlinc.28428 variants, one overlapping the second exon and another corresponding to a splice between first and third exon. All variations captured by the primer designs were quantified, suggesting that all these different variations predicted in silico exist biologically in MSCs. We confirmed most of the 
expression profiles obtained by k-mers quantification using RT-qPCR, notably the specificity of expression dependency on the MSCs tissular origin: over expression of Mlinc.28428 and 128022 in BM- and Ad-MSCs. Nevertheless, few exceptions, such as Mlinc.89912.1, present an enrichment in UC-MSCs not found in k-mers quantification. Moreover, the restricted expression to cells of mesodermal origin is replicated in our RT-qPCR results. We obtained similar observations with annotated candidates : overexpression of KRTAP1-5 and SMILR in BM-MSCs specifically, and of HOTAIR in UC- and BM-MSCs.

\subsection{In silico prediction of lncRNAs interactions and functions}

The relative specificity of selected Mlincs for mesenchymal cells could be an indication of their roles in MSCs function. The prediction of their possible function could therefore suggest their suitability as markers of MSCs' function potential. To this end, we explored assumptions on the function of Mlinc.28428.2, Mlinc.128022.2 and Mlinc.89912.1 candidates using different published methods. We first used bioinformatic tools based on machine learning and deep learning to decipher general characteristics of our candidates : FEELnc to assess coding potential, tarpMir to decipher "miRNA sponge" function, and LncADeep to analyse potential interactions with proteins. Only two of the 35 selected Mlincs and none of the 3 selected Mlincs with validated specificity were revealed as potentially coding RNAs with the majority being predicted as non-coding by FEELnc (33/35). None candidate had more than five target sites for a given miRNA, indicating a low probability of a "miRNA sponge" activity (Table S7). For the 3 retained Mlincs, predicted interacting proteins by LncADeep were submitted to Reactome (Table S8).

We noted a predicted interaction between Mlinc.28428.2 and Betacatenin (CTNNB1) as part of apoptosis-linked modules, 5'-3' Exoribonuclease 1, component of the CCR4-NOT complex, mRNA Decapping Enzyme 1B as part of the mRNA decapping and decay pathways. The interaction was also predicted with different mediators of RNA polymerase II transcription subunits (MED), ATP Binding Cassette Subfamily B Members as part of the PPARA activity linked to ER-stress [50], and Proteasome subunits for intracellular transport, response to hypoxia and cell cycle modules. Mlinc.128022 could interact with important genes like THY1 (CD90), NRF1 (mitochondria metabolism) with no module clearly highlighted. Mlinc.89912 could interact with tubulins, UBB (ubiquitin B), SMG6 nonsense mediated mRNA decay factor and ribosomes subunits (RPSX) proteins, (RPL24) for nonsense Mediated Decay (NMD), PINK1 (mitophagy) and finally MGMT, as part of the MGMT mediated DNA damage reversal module.

We further quantified expresssion of candidates by counting their specific k-mers in the entire FANTOM6 set of 154 Known-Downed (KD) annotated lncRNAs in human dermal fibroblasts (https://doi.org/10.1101/700864, not peer-reviewed). We selected the KD experiments where expression of the Mlincs was statistically differential when compared with controls. Particular attention was paid to KD lncRNAs with reported function(s) in bibliography, and to KD lncRNAs overlapping a gene with reported functions. Mlinc.28428.2 is down-regulated when JPX, SERTAD4-AS1, BOLA3-AS1, and SNRPD3 are KD, and over-expressed with the KD of PTCHD3P1, ERVK3.1, MEG3, among other lncRNAs without reported function (Figure 5A). Interestingly, interactions between p53 pathway and JPX [51], SNRPD3 [52] and MEG3 [53, 54]) respectively, have been previously reported. All these features converge on the hypothesis of a link between the function of Mlinc.28428, stress response, senescence and cellular maintenance. The implications of BOLA3 $[55,56])$ and PTCHD3P1 [57] in mitochondria homeostasis and glycolysis, the role of BOLA3 in stress response [58], the status of SERTAD4 as target of the YAP/TAZ pathway [59], vital pathway of stress response [60], and the role of MEG3 in aging [61], all reinforce 
this hypothesis.

Mlinc.128022.2 is down-regulated with the KD of FOXN3-AS1, A1BG-AS1, CD27-AS1, and FLVCR1-AS1 (Figure 5B). FOXN3 seems to be more than a regulator of cell cycle, it is also described as a regulator of osteogenesis in different cases of defective craniofacial development $[62,63]$. Moreover, the reported over-expression of FOXN3 during the early stages of MSCs osteodifferentiation [64], and downregulation of CD27-AS1 in MSCs of donors with bone fracture [65], allow us to hypothesise a possible function of Mlinc.128022 in bone remodelling and osteogenesis. In addition, both A1BG-AS1 and FLVCR1-AS have an influence in osteogenesis and cell differentiation. A recent study showed that A1BG-AS1 interacts with miR-216a and SMAD7 in suppressing hepatocellular carcinoma proliferation [66], both partners having an important role in the positive regulation of osteoblastic differentiation in mice [67] [68]. FLVR1 participates to resistance of oxydative stress by heme exportation in mouse MSCs [69], iron metabolism being closely linked with bone homeostasis, formation [70] and cell differentiation [71].

Finally, Mlinc.89912.1 is down-regulated after the KD of NEAT1-1 and PCAT6, and overexpressed when MFI2.AS1, CDKN2B.AS1 (or ANRIL) and MKLN1.AS2 are KD (Figure 5C). The manifest relations between cell proliferation and CDKN2B-AS1 [72, 73], MFI2 [74], MFI.AS1 [75], PCAT6 [76] and NEAT1 [77, 78], an combination with the between ones and DNA damage repair response, [79, 80] reinforces the prediction of a role of Mlinc.89912 in these mechanisms. Moreover, we explored RNAseq from chromatin, nucleus and cytoplasm subcellular compartments of fibroblastic cells in the FANTOM6 Dataset. Mlinc.28428 and Mlinc.128022 are enriched in at least cytoplasm, whereas Mlinc.89912 is enriched in free nucleus fraction suggesting interaction with nuclear component (Figure 5C).

\subsection{The single cell RNAseq: an emergent level of completion in marker search}

We analysed the single cell RNAseq data from 26071 adipose MSCs (Ad-MSCs) to assess the heterogeneity of the 3 Mlincs and to explore their expression at the single-cell level. No clear correlation between cell cycle and expression of our Mlincs was identified (Figure S9). We observed a high variability of the number of cells expressing the markers (Threshold $\geq 0.1$ ). 11 927/26 071 were Mlinc.28428-positives, 4944 were Mlinc.128022-positives, and 404 were Mlinc.89912-positives. For each Mlinc, we performed a differential test to decipher genes differentially expressed in AdMSCs Mlinc-positive and Mlinc-negative cells.

We found that Mlinc.28428-positive cells under-expressed H19 and PI16 (Figure 5A). These genes, that present a diversity of functions, are involved in stress mechanisms (oxydative response and shear stress), inflammation in fibroblasts and MSCs, and senescence pathways [81, 82, 83, 84]. Despite the low number of differentially expressed genes in Mlinc.28428-positive cells, their functional behaviour and their known targets suggest a pathway linked to stress response and senescence establishment that reinforce our previous assumptions on Mlinc.28428 function.

Mlinc.128022-positive cells are enriched in FTH1, TPM2, FTL and CD24 and present a lower expression in HMGN2, HMGB1, ODC1, PTTG1, BIRC5, EIF5A, MKI67, UBE2S, FGF5, HAS2AS1 (Figure 5B). A significant portion of these genes are linked to osteogenic properties of MSCs as previously observed with FANTOM analysis. The Mlinc.128022-positive cells have an increased expression of ferritin (light and heavy chains), major actor in iron metabolism in osteoblastic cell line [85], that is also involved in osteogenic differentiation [86] and osteogenic calcification [87]. Two genes, enriched in Mlinc.128022-positive cells are positively linked to the osteogenic 
differentiation potential of MSCs : the tropomyosin 2 (TPM2), downregulated when hMSCs were cultured in OS medium for the induction of osteoblasts at the calcification phase [88], and CD24 a membrane antigen recently proposed as a new marker for the sub-fraction of notochordal cells with increased differentiation capabilities [89]. In addition ODC1, under-represented in Mlinc.128022positive cells, inhibited the MSCs osteogenic differentiation [90, 91]. Finally, the decrease of FGF5, MKI67, BIRC5 (Survivin) and PTTG1 (securin) expressions, all linked to proliferation active phases of cell cycle, tend to show cell with arrested cell cycle. These data suggest that the expression profile of Mlinc.128022 positive cells indicate a subpopulation of undifferentiated osteogenic progenitors, probably in senescence or quiescence.

Mlinc.89912-positive cells are enriched in FGF5 and HIST1H4C (Figure 5C). FGF5 is a protein with mitogenic properties, identified as an oncogene, that facilitates cell proliferation in both autocrine [92] and paracrine manner [93]. HIST1H4C, the Histone Core number 4, is a cell cyclerelated gene. Modification of histone H4 (post-transcriptional or mutation) has been highlighted as important for Non-Homologous End-Joining (NHEJ) in yeast [94]. Its mutation cause genomic instability, resulting in increased apoptosis and cell cycle progression anomalies in zebrafish development. It reinforces our assumptions that Mlinc.89912 has a role in cell proliferation and DNA damage repair. In conclusion, the single cell RNAseq analysis enabled the observation of different features that characterise the phenotype of Mlincs positive cells and reinforced hypotheses on their functions previously observed through k-mers quantification.

\subsection{K-mers analysis of markers in functional cell situation}

Previously, we have presented a number of strategies to formulate hypotheses on the functions of an unannotated lncRNAs, suggesting directions of future experimental investigations. To evaluate the relevance of these strategies, we sought to quantifiy with specific k-mers search the expression of our Mlincs in MSCs in different conditions, linked to above mentioned findings: stress and senescence for Mlinc.28428.2, osteodifferentiation for Mlinc.128022.2 and cell cycle/proliferation for Mlinc.89912. We downloaded RNAseq data corresponding to the above-mentioned focus, described in Table S4.

As shown in Figure 6, we observed a statistically relevant increase of Mlinc.28428 expression in MSCs under replicative stress and in MSCs with CrisprCas9 depletion of genes with important role against senescence. In the Wang et al. study [95], MSCs senescence was observed with the $\mathrm{KO}$ of ATF6 and the stress induced with tunicamycin (endoplasmic reticulum stress) and late passage (replicative stress). Mlinc.28428 expression increased with tunicamycin treatment, late passage and ATF6 KO. The highest increase is observed in ATF6 KO MSCs associated with late passage condition.

In Fu et al. study [96], YAP, but not TAZ, was found to safeguard MSCs from cellular senescence as shown by $\mathrm{KO}$ experiments. Interestingly, YAP KO, but not TAZ, significantly increases the expression of Mlinc.28428.2. This would lead us to conclude that Mlinc.28428 is overexpressed in senescence and stress conditions, suggesting a role in one or both of these phenomena.

The change in Mlinc.128022 expression is strictly linked to osteodifferentiation conditions. Mlinc.128022 expression shows a relevant increase in MSCs exposed to fungal metabolite Cytochalasin D (CytoD). The cytoD is reported as a osteogenic stimulant in the concerned study [97]. Moreover, no expression variation was observed between MSCs and MSC-derived adipocytes from Wang et al. study, implying a role in adipodifferentiation. Agrawal Singh et al. have studied osteogenic MSCs differentiation [29], with a similar increase of Mlinc.128022 being observed after ten days. 
We then quantified the expression of Mlinc.89912 in a study that compare proliferating MSCs versus confluent MSCs [98, 99]. Our candidate was clearly over-expressed in proliferating cells, validating its capacity to mark the MSCs in proliferation. Moreover, its expression was not statistically modified when MSCs were exposed to EGF with pro-mitotic capabilities [100]. However Mlinc.89912 expression was reduced when IWR-1, an inhibitor of Beta-catenin nuclear translocation, that reduced the proliferation of MSCs, was added to the medium. The functional domains of these genes is summarised in table 1 and confirm the potential functional role suggested from FANTOM data: stress-related pathways for Mlinc.28428, MSCs differentiation with a presumed orientation in osteo-progenitors for Mlinc.128022 and a more restricted role in proliferation and DNA repair for Mlinc.89912.

\section{Discussion}

With recent evolution of omics analysis, the landscape of biomarkers has been extended beyond known genes to the exploration of the unexplored transcriptome. This potential has been assessed in pathological conditions but to a lesser extent in cell-specific conditions, where this new pool of potential markers could be used to identify less well-characterised cells and hence predict their function. In this article, we propose an integrated procedure and strategies to identify the best markers (annotated or not) in a cell-specific condition, and predict their potential functions, primarily from RNA sequencing data (Figure 1). RNAseq facilitates the creation of large lncRNA catalogues $[8,101]$ through the total catalogue of lncRNAs remains incomplete given the diversity of biological entities and lncRNAs specific expression in non-pathological, cell-specific conditions. The creation of a "home-made" catalogue associated with a specific condition remains the best way to assess the full diversity of potential biomarkers in a cell, rather than resorting to a global catalogue made from diverse samples. To give an idea of the completeness of such a focused lncRNA catalogue when compared to a global one, Jiang et al. recently published "an expanded landscape of human long non-coding RNA" with 25000 new lncRNAs from normal and tumor tissues, whereas in our focused analysis only $50 \%$ of our 35 selected MSClinc can be found in this collection [101].

Futhermore, providing new candidates of good quality to improve lncRNA collection remains a complex task. As could be expected, the raw catalogue in our study contains predictions of disparate quality observed with a large number of mono-exonic transcripts. Without any filter, $a b$ initio methods are insufficient to adequately reconstruct full length transcripts. The usage of longread sequencing has been particularly effective in helping to validate our predictions. Given the benefits of full-length RNA sequence, long-read RNAseq should become the standard for lncRNA validation. A specific lncRNA can be the one presenting the most relevant properties after in silico analysis. The first task remain the identification of the more specific markers for a given cell type, task that present differences from classic comparative analysis. The MSCs markers proposed in the past were determined through a simple comparison between MSCs of a certain origin with negative cell whose types are either unique or few in number.

Historically, MSCs have been compared to bone marrow haematopoietic stem cells. However, our initial RNAseq analysis revealed that all potential MSCs markers proposed in the past are expressed in at least one other non-mesenchymatous cells type, and so do not constitute exclusive MSCs markers at the transcriptome level. Even if all cell types cannot be investigated, the diversity of the negative cell set is a critical criterion in selecting the most specific transcripts. In keeping with this idea, we then restricted the list of potential biomarkers with an enrichment step based 
Table 1: Summary of functional investigation results

\begin{tabular}{|c|c|c|c|c|c|}
\hline Mlinc & $\begin{array}{l}\text { Predicted } \\
\text { RNA-Protein } \\
\text { interactions } \\
\text { (lncADeep) }\end{array}$ & $\begin{array}{l}\text { subcompartme } \\
\text { enrichment }\end{array}$ & $\begin{array}{l}\text { itANTOM6 } \\
\text { expr. changes }\end{array}$ & $\begin{array}{l}\text { Diff. genes in } \\
\text { positive cells }\end{array}$ & $\begin{array}{l}\text { K-mers inves- } \\
\text { tigations }\end{array}$ \\
\hline "Mlinc.28428 & $\begin{array}{l}\text { apoptosis, } \\
\text { mRNA de- } \\
\text { cay, PPARA } \\
\text { activity, in- } \\
\text { tracellular } \\
\text { transport, } \\
\text { response to } \\
\text { hypoxia and } \\
\text { cell cycle }\end{array}$ & $\begin{array}{l}\text { Chromatin, } \\
\text { cytoplasm }\end{array}$ & $\begin{array}{l}\text { BOLA3- } \\
\text { AS1, JPX, } \\
\text { SERTAD4- } \\
\text { AS1, } \\
\text { PTCHD3P1, } \\
\text { ERVK3.1, } \\
\text { SNRPD3, } \\
\text { MEG3 }\end{array}$ & H19 - PI16 - & $\begin{array}{l}\text { Stress, senes- } \\
\text { cence }\end{array}$ \\
\hline Mlinc.128022 & THY1, NRF1 & $\begin{array}{l}\text { Chromatin, } \\
\text { cytoplasm }\end{array}$ & $\begin{array}{l}\text { FOXN3-AS1, } \\
\text { A1BG-AS1, } \\
\text { CD27-AS1, } \\
\text { FLVCR1-AS1 }\end{array}$ & $\begin{array}{l}\text { FTH1, } \\
\text { TPM2, } \\
\text { FTL, CD24, } \\
\text { HMGN2, } \\
\text { HMGB1, } \\
\text { ODC1, } \\
\text { PTTG1, } \\
\text { BIRC5, } \\
\text { EIF5A, } \\
\text { MKI67, } \\
\text { UBE2S, } \\
\text { FGF5, HAS2- } \\
\text { AS1 }\end{array}$ & $\begin{array}{l}\text { Osteodiff., } \\
\text { stress }\end{array}$ \\
\hline Mlinc.89912 & $\begin{array}{l}\text { MGMT- } \\
\text { mediated } \\
\text { DNA dam- } \\
\text { age reversal, } \\
\text { Nonsense } \\
\text { Mediated De- } \\
\text { cay, Tubulin } \\
\text { metabolism }\end{array}$ & $\begin{array}{l}\text { nucleus } \\
\text { (free), cyto- } \\
\text { plasm }\end{array}$ & $\begin{array}{l}\text { NEAT1_1, } \\
\text { PCAT6, } \\
\text { MFI2.AS1, } \\
\text { MKLN1.AS2, } \\
\text { CDKN2B.AS1 }\end{array}$ & $\begin{array}{l}\text { FGF5 and } \\
\text { HIST1H4C }\end{array}$ & Proliferation \\
\hline
\end{tabular}

on a differential expression comparing BM-MSCs to other cells including mainly stem cells, as well as differentiated cells of various lineages (lymphocytes, macrophages, primary chondrocytes, hepatocytes and neurons). In the enriched list, the overexpressed annotated genes contained members of MSC-related pathways as well as the ISCT markers. This result supported the MSCs characterisation made by the original authors [13], thus validating the identity of MSCs used for this RNAseq analysis with the currently defined criteria. The problem with classical differential 
analysis used on diverse "non-MSC" group is that all the "non-MSC" group is considered to be homogeneous. As a result, candidates with positive expression in small cell groups could pass statistical test, creating false positives. For this kind of differential analysis, we propose to select the most discriminating transcripts by feature selection, a machine learning methodology, that reduces the number of non-discriminating candidates after selection. We used feature selection through Boruta, a method based on random forest, to retain the top 35 of the most relevant MSCs signature for annotated genes, Mlincs and Mloancs separately. Putting aside our initial focus on unannotated lncRNAs, different annotated lncRNAs or coding genes with interesting profiles were also selected by feature selection : among them, KRTAP1-5 have been exclusively studied in BM-MSCs [102], where its preferential expression was validated by our results. These discoveries can bring new features concerning these genes and suggest directions for future investigations concerning their impact on the MSCs.

However, a marker is classically considered as specific on condition that its positive expression cannot be observed in any other cell type. Therefore, the expression of these potential markers should be explored in an entire RNAseq database to further validate its specificity. The exploration of a wide set of RNAseq data as proposed by ENCODE including a diversified set of primary and stem cells could support or invalidate the specificity of potential markers. In order to assess the expression of Mlincs candidates in a large number of samples, we used a signature for each candidate, extracting specific 31nt k-mers from their sequences. The specific k-mers extraction was made using Kmerator software. These k-mers were then quantified in the ENCODE human RNAseq databaseThe new and simplified procedure based on k-mers counting and large scale RNAseq exploration has the following advantages: i) a direct textual search that requires less time and CPU resources than classical methods, ii) a restricted set of lncRNAs supported by different results in the biological (wet) and in silico level (RNAseq data). The counterpart of the extensive vision of marker expression is that we observe a limit of specificity among our best candidates. We observed expression in fibroblasts, in close primary cells of common embryonic origin like smooth muscle cells (SMC) and other tissue-specific fibroblastic cells. Other tissue resident fibroblastic cells like skeletal muscle satellite cells, pre-adipocytes, and fibroblasts from different sources, especially dermis, express our selected MSClincs markers. The question of the differences between MSCs and related cell types is crucial to the issue. Specifically, the differences between MSCs and fibroblasts remain a subject of debate [103, 12]. According to the ISCT statement, no phenotypical differences have been reported between fibroblasts of different sources and adult MSCs [104], suggesting a hypothesis of a uniform cell type that show functional variation depending on the tissue source. Our results support this idea: distinguishing MSCs from fibroblast with only few positive markers remains a complicated task.

Moreover we observe low to medium expression of our candidates in close cell type from the same embryonic origin such as muscular cells and smooth muscle cells (SMC). This could be due to a shared phenotype between cells with close embryonic origin. Common markers between MSCs and SMCs have already been described. Notably, MSCs can express similar levels of SMC markers such as alpha-actin [105, 106]. Moreover Kumar et al. [107] determined that MSCs, pericytes, and SMCs could have the same mesenchymo-angioblast progenitor and that SMCs share a certain plasticity with MSCs as they can be differentiated in chondrocyte-like and beige adipocytes or myofibroblasts. However, a lot of cell types in ENCODE have not been actively sorted by expression of their respective surface markers, and fibroblast contamination is a classical feature in primary cell culture. We should not therefore exclude the possibility of fibroblast contamination when investigating marker for MSCs by bulk omics technology. Given this, single-cell RNAseq could be the best solution to identify the source of marker expression in counterpart cells. 
To conclude, our extensive cell type comparison shows that the discovery of a marker of MSCs as distinct cell type is not plausible. After deepening our own research on MSCs biomarkers at the annotated and unannotated level, we were unable to find a marker that could simultaneously i) distinguish MSCs to close or homologuous cell types (fibroblasts, satellite cells, SMCs) ii) be present in all MSCs types iii) distinguish MSCs from more characterised cell types (Hematopoietic lineage, neurones etc). Our results suggest, like other studies, a strong proximity between MSCs, fibroblast and mesodermal cell types.

More than a marker of MSCs, candidates extracted by our method could be used to explore important features in MSCs biology and therefore warrant investigation into their function, assuming that the specificity of RNA for a cell type can highlight its importance in cell activity. Even if the functional invalidation stands as the principal method to efficiently determine the function of a lncRNA, its expression and co-expression with known genes can potentially characterise a function or an intrinsic state of a cell type, particularly for MSCs with reported diversity of states and function (ex : differentiation, immunomodulation, senescence, proliferation...). In our opinion, it is vital that during the creation of a catalogue of lncRNAs, a restricted set of selected biomarkers should be studied more intensively, both in term of specificity and functions. Assumptions on functional domains, where lncRNAs could act, could increase the relevance and visibility of discovered lncRNAs, and far from the bioinformatics implications, encourage future biological investigations. We decided to investigate the three selected MSClincs, validated by k-mers search, RT-qPCR and long-read sequencing, in term of biological impact with complementary in silico experimental approaches. We propose to use different in silico strategies, depending on the amount and diversity of the available data. The analysis confirms the non-coding potential of candidates and indicates a low probability of "miRNA sponge" activity. However, protein potential interaction results give interesting paths that were then investigated by complementary exploration. The k-mers quantification permits a naive high throughput exploration of numerous RNAseq data, simultaneously exploring potential functions and specificity to assess their potential. Instead of different cells, each candidate's expression was quantified in MSCs in different experimental conditions. FANTOM6 data recently offered a pilot about lncRNAs functional investigation, with a high-throughput invalidation of 154 lncRNAs and coding genes in fibroblasts and their RNAseq counterpart added to phenotypical observations. The utilisation of co-expressions between knock-out genes and candidates lncRNAs remains an efficient way to decipher lncRNAs function, provided number of KD genes is high. Moreover, the availability of recent single cell data of MSCs have been a good complement to lncRNAs functional investigation.

Using scRNAseq from Ad-MSCs [19], we observed that our markers are not expressed in all cells but constitute different subpopulations with different levels of rarity in Ad-MSCs. FANTOM6 and single-cell analysis could permit tracing three components of these states : stress inducible cells, lineage commited osteogenic progenitors and proliferating cells. Globally, we observed a global concordance of the results between the different strategies used for functional prediction. Mlinc.28428 has concomitant expression with genes related to the stress response pathway. Mlinc.28428 could be a good target for treatment to study the senescence process, age pathologies or stress response. Mlinc.128022 potentially interacts with THY1 (CD90) and has co-occurences with genes linked to osteoprogenitors and cell differentiation. The k-mers search highlights its participation in MSCs' osteo-differentiation. Finally, Mlinc.89912 potentially interacts with damage repair and RNA decay, and tubulin metabolism, all linked to cell proliferation and cell cycle. Moreover, the subcompartment enrichment corresponds to this prediction: Mlinc.89912.1 is the only candidate to have possible interactions with DNA-repair system, a hypothesis corresponding to his observed enrichment in the nucleus. A final selection of bulk RNAseq of MSCs in specific biological con- 
ditions allowed confirmation of our initial assumptions, showing that the different strategies we propose could be used to give relevant indication of the lncRNAs' functions. These results show that a lncRNA selected by its expression specificity has a high probability of being part of a functional mechanism.

In conclusion, we have predicted genes and lncRNAs enriched in MSCs and proposed several selection steps including feature selection (machine learning), large scale signature search, RTqPCR validation, in silico tools and single cell analysis. We present the application of a new way of quantification in RNAseq : The specific k-mers search could be used as a naive information in lncRNA catalogue creation. The strategies presented here are transferable to other cell types and different studies while the specificity and functional assumption present a significant potential in long non-coding transcriptome exploration. We present three lncRNAs markers of bone marrow and adipose MSCs that passed all selection steps and present interesting features: Mlinc.28428.2, Mlinc.128022.2 and Mlinc.89912.1. These markers could be used by the scientific community as potential targets for functional biological experiments on MSCs, with pre-indications of potential functions to orientate the experiments, and finally initiate the objective of transition between informatical problematics and cell biology.

\section{$4 \quad$ Funding}

Grant information: this work was supported by the Agence Nationale de la recherche for the projects "Computational Biology Institute" and "Transipedia" '[grant numbers 18-CE45-0020-02, ANR-10-INBS-09]' and the Canceropole Grand-Sud-Ouest Trans-kmer" project '[grant number 2017-EM24]'.

\section{Acknowledgements}

We thank for their generous gifts, G.Carnac for myoblasts, M.Le Quintrec-Donnette for HUVECs, E. Sanchez for dermal fibroblasts, D. Noel and ML. Vignais for mesenchymal stromal cells, C. Crozet for IPS, S. Gerbal and M. Daujat for hepatocytes. We thank Philippe Clair for his advice on qPCR, the qPHD plateform, Montpellier GenomiX and Jean-Marc Holder (SeqOne) for text corrections.

\subsubsection{Conflict of interest statement.}

The authors declare that they have no competing interests.

\section{References}

[1] Gloss, B. S. and Dinger, M. E. (January, 2016) The specificity of long noncoding RNA expression. Biochimica et Biophysica Acta (BBA) - Gene Regulatory Mechanisms, 1859(1), $16-22$.

[2] Meseure, D., Drak Alsibai, K., Nicolas, A., Bieche, I., and Morillon, A. (2015) Long Noncoding RNAs as New Architects in Cancer Epigenetics, Prognostic Biomarkers, and Potential Therapeutic Targets. BioMed Research International, 2015. 
[3] Bouckenheimer, J., Assou, S., Riquier, S., Hou, C., Philippe, N., Sansac, C., LavabreBertrand, T., Commes, T., Lematre, J.-M., Boureux, A., and Vos, J. D. (September, 2016) Long non-coding RNAs in human early embryonic development and their potential in ART. Human Reproduction Update,.

[4] Li, L. and Chang, H. Y. (October, 2014) Physiological roles of long noncoding RNAs: Insights from knockout mice. Trends in cell biology, 24(10), 594-602.

[5] Dhamija, S. and Diederichs, S. (2016) From junk to master regulators of invasion: lncRNA functions in migration, EMT and metastasis. International Journal of Cancer, 139(2), 269280 .

[6] Li, X. and Li, N. (December, 2018) LncRNAs on guard. International Immunopharmacology, 65, 60-63.

[7] Morillon, A. and Gautheret, D. (June, 2019) Bridging the gap between reference and real transcriptomes. Genome Biology, 20.

[8] Uszczynska-Ratajczak, B., Lagarde, J., Frankish, A., Guig, R., and Johnson, R. (September, 2018) Towards a complete map of the human long non-coding RNA transcriptome. Nature reviews. Genetics, 19(9), 535-548.

[9] James, A. R., Schroeder, M. P., Neumann, M., Bastian, L., Eckert, C., Gkbuget, N., Tanchez, J. O., Schlee, C., Isaakidis, K., Schwartz, S., Burmeister, T., von Stackelberg, A., Rieger, M. A., Gllner, S., Horstman, M., Schrappe, M., Kirschner-Schwabe, R., Brggemann, M., Mller-Tidow, C., Serve, H., Akalin, A., and Baldus, C. D. (January, 2019) Long non-coding RNAs defining major subtypes of B cell precursor acute lymphoblastic leukemia. Journal of Hematology \& Oncology, 12.

[10] Liu, X., Ma, Y., Yin, K., Li, W., Chen, W., Zhang, Y., Zhu, C., Li, T., Han, B., Liu, X., Wang, S., and Zhou, Z. (June, 2019) Long non-coding and coding RNA profiling using strand-specific RNA-seq in human hypertrophic cardiomyopathy. Scientific Data, 6(1), 1-7.

[11] Lv, F.-J., Tuan, R. S., Cheung, K. M., and Leung, V. Y. (June, 2014) Concise Review: The Surface Markers and Identity of Human Mesenchymal Stem Cells. STEM CELLS, 32(6), 1408-1419.

[12] Soundararajan, M. and Kannan, S. (December, 2018) Fibroblasts and mesenchymal stem cells: Two sides of the same coin?. Journal of Cellular Physiology, 233(12), 9099-9109.

[13] Dominici, M., Le Blanc, K., Mueller, I., Slaper-Cortenbach, I., Marini, F., Krause, D., Deans, R., Keating, A., Prockop, D., and Horwitz, E. (2006) Minimal criteria for defining multipotent mesenchymal stromal cells. The International Society for Cellular Therapy position statement. Cytotherapy, 8(4), 315-317.

[14] Fitzsimmons, R. E. B., Mazurek, M. S., Soos, A., and Simmons, C. A. (August, 2018) Mesenchymal Stromal/Stem Cells in Regenerative Medicine and Tissue Engineering. Stem Cells International, 2018.

[15] Olsen, T. R., Ng, K. S., Lock, L. T., Ahsan, T., and Rowley, J. A. (June, 2018) Peak MSCAre We There Yet?. Frontiers in Medicine, 5. 
[16] Tye, C. E., Gordon, J. A. R., Martin-Buley, L. A., Stein, J. L., Lian, J. B., and Stein, G. S. (March, 2015) Could lncRNAs be the missing links in control of mesenchymal stem cell differentiation?. Journal of Cellular Physiology, 230(3), 526-534.

[17] Kalwa, M., Hnzelmann, S., Otto, S., Kuo, C.-C., Franzen, J., Joussen, S., Fernandez-Rebollo, E., Rath, B., Koch, C., Hofmann, A., Lee, S.-H., Teschendorff, A. E., Denecke, B., Lin, Q., Widschwendter, M., Weinhold, E., Costa, I. G., and Wagner, W. (December, 2016) The lncRNA HOTAIR impacts on mesenchymal stem cells via triple helix formation. Nucleic Acids Research, 44(22), 10631-10643.

[18] Song, W., Gu, W., Qian, Y., Ma, X., Mao, Y., and Liu, W. (2015) Identification of long non-coding RNA involved in osteogenic differentiation from mesenchymal stem cells using RNA-Seq data. Genetics and Molecular Research, 14(4), 18268-18279.

[19] Liu, X., Xiang, Q., Xu, F., Huang, J., Yu, N., Zhang, Q., Long, X., and Zhou, Z. (February, 2019) Single-cell RNA-seq of cultured human adipose-derived mesenchymal stem cells. Scientific Data, 6, 190031.

[20] Peffers, M. J., Collins, J., Fang, Y., Goljanek-Whysall, K., Rushton, M., Loughlin, J., Proctor, C., and Clegg, P. D. (2016) Age-related changes in mesenchymal stem cells identified using a multi-omics approach. European Cells $\&$ Materials, 31, 136-159.

[21] Philippe, N., Salson, M., Commes, T., and Rivals, E. (2013) CRAC: an integrated approach to the analysis of RNA-seq reads. Genome Biology, 14, R30.

[22] Pertea, M., Pertea, G. M., Antonescu, C. M., Chang, T.-C., Mendell, J. T., and Salzberg, S. L. (March, 2015) StringTie enables improved reconstruction of a transcriptome from RNA-seq reads. Nature Biotechnology, 33(3), 290-295.

[23] Quinlan, A. R. and Hall, I. M. (March, 2010) BEDTools: a flexible suite of utilities for comparing genomic features. Bioinformatics, 26(6), 841-842.

[24] Ilott, N. E. and Ponting, C. P. (September, 2013) Predicting long non-coding RNAs using RNA sequencing. Methods (San Diego, Calif.), 63(1), 50-59.

[25] Li, H. (July, 2016) Minimap and miniasm: fast mapping and de novo assembly for noisy long sequences. Bioinformatics, 32(14), 2103-2110.

[26] Bray, N. L., Pimentel, H., Melsted, P., and Pachter, L. (May, 2016) Near-optimal probabilistic RNA-seq quantification. Nature Biotechnology, 34(5), 525-527.

[27] Pimentel, H., Bray, N. L., Puente, S., Melsted, P., and Pachter, L. (July, 2017) Differential analysis of RNA-seq incorporating quantification uncertainty. Nature Methods, 14(7), 687690.

[28] Kursa, M. B., Jankowski, A., and Rudnicki, W. R. (December, 2010) Boruta - A System for Feature Selection. Fundam. Inf., 101(4), 271-285.

[29] Agrawal Singh, S., Lerdrup, M., Gomes, A.-L. R., van de Werken, H. J., Vilstrup Johansen, J., Andersson, R., Sandelin, A., Helin, K., and Hansen, K. PLZF targets developmental enhancers for activation during osteogenic differentiation of human mesenchymal stem cells. eLife, 8. 
[30] Yang, C., Yang, L., Zhou, M., Xie, H., Zhang, C., Wang, M. D., and Zhu, H. (November, 2018) LncADeep: an ab initio lncRNA identification and functional annotation tool based on deep learning. Bioinformatics, 34(22), 3825-3834.

[31] Ding, J., Li, X., and Hu, H. (September, 2016) TarPmiR: a new approach for microRNA target site prediction. Bioinformatics, 32(18), 2768-2775.

[32] Wucher, V., Legeai, F., Hdan, B., Rizk, G., Lagoutte, L., Leeb, T., Jagannathan, V., Cadieu, E., David, A., Lohi, H., Cirera, S., Fredholm, M., Botherel, N., Leegwater, P. A. J., Le Bguec, C., Fieten, H., Johnson, J., Alfldi, J., Andr, C., Lindblad-Toh, K., Hitte, C., and Derrien, T. (May, 2017) FEELnc: a tool for long non-coding RNA annotation and its application to the dog transcriptome. Nucleic Acids Research, 45(8), e57-e57.

[33] Stuart, T., Butler, A., Hoffman, P., Hafemeister, C., Papalexi, E., Mauck, W. M., Hao, Y., Stoeckius, M., Smibert, P., and Satija, R. (June, 2019) Comprehensive Integration of Single-Cell Data. Cell, 177(7), 1888-1902.e21.

[34] Butler, A., Hoffman, P., Smibert, P., Papalexi, E., and Satija, R. (May, 2018) Integrating single-cell transcriptomic data across different conditions, technologies, and species. Nature Biotechnology, 36(5), 411-420.

[35] Johnson, W. E., Li, C., and Rabinovic, A. (January, 2007) Adjusting batch effects in microarray expression data using empirical Bayes methods. Biostatistics, 8(1), 118-127.

[36] Hahne, F. and Ivanek, R. (2016) Visualizing Genomic Data Using Gviz and Bioconductor. In Math, E. and Davis, S., (eds.), Statistical Genomics: Methods and Protocols, Methods in Molecular Biology pp. 335-351 Springer New York New York, NY.

[37] Djouad, F., Bony, C., Hupl, T., Uz, G., Lahlou, N., Louis-Plence, P., Apparailly, F., Canovas, F., Rme, T., Sany, J., Jorgensen, C., and Nol, D. (2005) Transcriptional profiles discriminate bone marrow-derived and synovium-derived mesenchymal stem cells. Arthritis Research \&6 Therapy, 7(6), R1304-R1315.

[38] Kitzmann, M., Bonnieu, A., Duret, C., Vernus, B., Barro, M., LaoudjChenivesse, D., Verdi, J. M., and Carnac, G. (2006) Inhibition of Notch signaling induces myotube hypertrophy by recruiting a subpopulation of reserve cells. Journal of Cellular Physiology, 208(3), 538-548.

[39] Pichard, L., Raulet, E., Fabre, G., Ferrini, J. B., Ourlin, J.-C., and Maurel, P. (2006) Human Hepatocyte Culture. In Phillips, I. R. and Shephard, E. A., (eds.), Cytochrome P450 Protocols, Methods in Molecular Biology pp. 283-293 Humana Press Totowa, NJ.

[40] Livak, K. J. and Schmittgen, T. D. (December, 2001) Analysis of Relative Gene Expression Data Using Real-Time Quantitative PCR and the 2CT Method. Methods, 25(4), 402-408.

[41] Niazi, F. and Valadkhan, S. (April, 2012) Computational analysis of functional long noncoding RNAs reveals lack of peptide-coding capacity and parallels with 3 UTRs. $R N A, \mathbf{1 8}(4)$, $825-843$.

[42] Wang, Y., Xu, T., He, W., Shen, X., Zhao, Q., Bai, J., and You, M. (January, 2018) Genome-wide identification and characterization of putative lncRNAs in the diamondback moth, Plutella xylostella (L.). Genomics, 110(1), 35-42. 
[43] Cagirici, H. B., Alptekin, B., and Budak, H. (September, 2017) RNA Sequencing and Coexpressed Long Non-coding RNA in Modern and Wild Wheats. Scientific Reports, 7.

[44] Salari, R., Aksay, C., Karakoc, E., Unrau, P. J., Hajirasouliha, I., and Sahinalp, S. C. (May, 2009) smyRNA: A Novel Ab Initio ncRNA Gene Finder. PLoS ONE, 4(5).

[45] Gu, Q., Tian, H., Zhang, K., Chen, D., Chen, D., Wang, X., and Zhao, J. (2018) Wnt5a/FZD4 Mediates the Mechanical Stretch-Induced Osteogenic Differentiation of Bone Mesenchymal Stem Cells. Cellular Physiology and Biochemistry, 48(1), 215-226.

[46] Diederichs, S., Tonnier, V., Mrz, M., Dreher, S. I., Geisbsch, A., and Richter, W. (October, 2019) Regulation of WNT5A and WNT11 during MSC in vitro chondrogenesis: WNT inhibition lowers BMP and hedgehog activity, and reduces hypertrophy. Cellular and molecular life sciences: CMLS, 76(19), 3875-3889.

[47] Bermeo, S., Vidal, C., Zhou, H., and Duque, G. (2015) Lamin A/C Acts as an Essential Factor in Mesenchymal Stem Cell Differentiation Through the Regulation of the Dynamics of the Wnt/-Catenin Pathway. Journal of Cellular Biochemistry, 116(10), 2344-2353.

[48] Chung, K.-M., Hsu, S.-C., Chu, Y.-R., Lin, M.-Y., Jiaang, W.-T., Chen, R.-H., and Chen, X. (February, 2014) Fibroblast Activation Protein (FAP) Is Essential for the Migration of Bone Marrow Mesenchymal Stem Cells through RhoA Activation. PLoS ONE, 9(2).

[49] Ruffl, F., Audoux, J., Boureux, A., Beaumeunier, S., Gaillard, J.-B., Bou Samra, E., Megarbane, A., Cassinat, B., Chomienne, C., Alves, R., Riquier, S., Gilbert, N., Lemaitre, J.-M., Bacq-Daian, D., Boug, A. L., Philippe, N., and Commes, T. (December, 2017) New chimeric RNAs in acute myeloid leukemia. F1000Research, 6, 1302.

[50] Krieken, S. E. v. d., Popeijus, H. E., Mensink, R. P., and Plat, J. (2017) Link Between ER-Stress, PPAR-Alpha Activation, and BET Inhibition in Relation to Apolipoprotein A-I Transcription in HepG2 Cells. Journal of Cellular Biochemistry, 118(8), 2161-2167.

[51] Delbridge, A. R. D., Kueh, A. J., Ke, F., Zamudio, N. M., El-Saafin, F., Jansz, N., Wang, G.-Y., Iminitoff, M., Beck, T., Haupt, S., Hu, Y., May, R. E., Whitehead, L., Tai, L., Chiang, W., Herold, M. J., Haupt, Y., Smyth, G. K., Thomas, T., Blewitt, M. E., Strasser, A., and Voss, A. K. (April, 2019) Loss of p53 Causes Stochastic Aberrant X-Chromosome Inactivation and Female-Specific Neural Tube Defects. Cell Reports, 27(2), 442-454.e5.

[52] Siebringvan Olst, E., Blijlevens, M., de Menezes, R. X., van der MeulenMuileman, I. H., Smit, E. F., and van Beusechem, V. W. (May, 2017) A genomewide siRNA screen for regulators of tumor suppressor p53 activity in human nonsmall cell lung cancer cells identifies components of the RNA splicing machinery as targets for anticancer treatment. Molecular Oncology, 11(5), 534-551.

[53] Zhou, Y., Zhong, Y., Wang, Y., Zhang, X., Batista, D. L., Gejman, R., Ansell, P. J., Zhao, J., Weng, C., and Klibanski, A. (August, 2007) Activation of p53 by MEG3 Non-coding RNA. Journal of Biological Chemistry, 282(34), 24731-24742.

[54] Uroda, T., Anastasakou, E., Rossi, A., Teulon, J.-M., Pellequer, J.-L., Annibale, P., Pessey, O., Inga, A., Chilln, I., and Marcia, M. (September, 2019) Conserved Pseudoknots in lncRNA MEG3 Are Essential for Stimulation of the p53 Pathway. Molecular Cell, 75(5), 982-995.e9. 
[55] Haack, T. B., Rolinski, B., Haberberger, B., Zimmermann, F., Schum, J., Strecker, V., Graf, E., Athing, U., Hoppen, T., Wittig, I., Sperl, W., Freisinger, P., Mayr, J. A., Strom, T. M., Meitinger, T., and Prokisch, H. (2013) Homozygous missense mutation in BOLA3 causes multiple mitochondrial dysfunctions syndrome in two siblings. Journal of Inherited Metabolic Disease, 36(1), 55-62.

[56] Yu Qiujun, Tai Yi-Yin, Tang Ying, Zhao Jingsi, Negi Vinny, Culley Miranda K., Pilli Jyotsna, Sun Wei, Brugger Karin, Mayr Johannes, Saggar Rajeev, Saggar Rajan, Wallace W. Dean, Ross David J., Waxman Aaron B., Wendell Stacy G., Mullett Steven J., Sembrat John, Rojas Mauricio, Khan Omar F., Dahlman James E., Sugahara Masataka, Kagiyama Nobuyuki, Satoh Taijyu, Zhang Manling, Feng Ning, Gorcsan John, Vargas Sara O., Haley Kathleen J., Kumar Rahul, Graham Brian B., Langer Robert, Anderson Daniel G., Wang Bing, Shiva Sruti, Bertero Thomas, and Chan Stephen Y. (May, 2019) BOLA (BolA Family Member 3) Deficiency Controls Endothelial Metabolism and Glycine Homeostasis in Pulmonary Hypertension. Circulation, 139(19), 2238-2255.

[57] Wang, J. and Li, K. (April, 2018) AB042. P013. LncRNAPTCHD3P1 enhances chemosensitivity of gemcitabine in pancreatic cancer and inhibits cancer cell proliferation and metastasis via inhibiting Warburg effect. Annals of Pancreatic Cancer, 1(4).

[58] Qin, L., Wang, M., Zuo, J., Feng, X., Liang, X., Wu, Z., and Ye, H. (April, 2015) Cytosolic BolA Plays a Repressive Role in the Tolerance against Excess Iron and MV-Induced Oxidative Stress in Plants. PLoS ONE, 10(4).

[59] Kitajima, S., Asahina, H., Chen, T., Guo, S., Quiceno, L. G., Cavanaugh, J. D., Merlino, A. A., Tange, S., Terai, H., Kim, J. W., Wang, X., Zhou, S., Xu, M., Wang, S., Zhu, Z., Thai, T. C., Takahashi, C., Wang, Y., Neve, R., Stinson, S., Tamayo, P., Watanabe, H., Kirschmeier, P. T., Wong, K.-K., and Barbie, D. A. (September, 2018) Overcoming Resistance to Dual Innate Immune and MEK Inhibition Downstream of KRAS. Cancer cell, 34(3), 439-452.e6.

[60] Raj, N. and Bam, R. (August, 2019) Reciprocal Crosstalk Between YAP1/Hippo Pathway and the p53 Family Proteins: Mechanisms and Outcomes in Cancer. Frontiers in Cell and Developmental Biology, 7.

[61] He, J., Tu, C., and Liu, Y. (2018) Role of lncRNAs in aging and age-related diseases. AGING MEDICINE, 1(2), 158-175.

[62] Schuff, M., Rssner, A., Wacker, S. A., Donow, C., Gessert, S., and Knchel, W. (2007) FoxN3 is required for craniofacial and eye development of Xenopus laevis. Developmental Dynamics, 236(1), 226-239.

[63] Samaan, G., Yugo, D., Rajagopalan, S., Wall, J., Donnell, R., Goldowitz, D., Gopalakrishnan, R., and Venkatachalam, S. (September, 2010) Foxn3 is essential for craniofacial development in mice and a putative candidate involved in human congenital craniofacial defects. Biochemical and Biophysical Research Communications, 400(1), 60-65.

[64] Brum, A. M., van de Peppel, J., van der Leije, C. S., Schreuders-Koedam, M., Eijken, M., van der Eerden, B. C. J., and van Leeuwen, J. P. T. M. (October, 2015) Connectivity Mapbased discovery of parbendazole reveals targetable human osteogenic pathway. Proceedings of the National Academy of Sciences of the United States of America, 112(41), 12711-12716. 
[65] del Real, A., Prez-Campo, F. M., Fernndez, A. F., Saudo, C., Ibarbia, C. G., Prez-Nez, M. I., Criekinge, W. V., Braspenning, M., Alonso, M. A., Fraga, M. F., and Riancho, J. A. (December, 2016) Differential analysis of genome-wide methylation and gene expression in mesenchymal stem cells of patients with fractures and osteoarthritis. Epigenetics, 12(2), $113-122$.

[66] Bai, J., Yao, B., Wang, L., Sun, L., Chen, T., Liu, R., Yin, G., Xu, Q., and Yang, W. (2019) lncRNA A1BG-AS1 suppresses proliferation and invasion of hepatocellular carcinoma cells by targeting miR-216a-5p. Journal of Cellular Biochemistry, 120(6), 10310-10322.

[67] Li, N., Lee, W. Y.-W., Lin, S.-E., Ni, M., Zhang, T., Huang, X.-R., Lan, H.-Y., and Li, G. (October, 2014) Partial loss of Smad7 function impairs bone remodeling, osteogenesis and enhances osteoclastogenesis in mice. Bone, 67, 46-55.

[68] Vishal, M., Vimalraj, S., Ajeetha, R., Gokulnath, M., Keerthana, R., He, Z., Partridge, N. C., and Selvamurugan, N. (2017) MicroRNA-590-5p Stabilizes Runx2 by Targeting Smad7 During Osteoblast Differentiation. Journal of Cellular Physiology, 232(2), 371-380.

[69] Nowak, W. N., Taha, H., Kachamakova-Trojanowska, N., Stpniewski, J., Markiewicz, J. A., Kusienicka, A., Szade, K., Szade, A., Bukowska-Strakova, K., Hajduk, K., Klska, D., Kopacz, A., Grochot-Przczek, A., Barthenheier, K., Cauvin, C., Dulak, J., and Jzkowicz, A. (October, 2017) Murine Bone Marrow Mesenchymal Stromal Cells Respond Efficiently to Oxidative Stress Despite the Low Level of Heme Oxygenases 1 and 2. Antioxidants \& Redox Signaling, 29(2), 111-127.

[70] Balogh, E., Paragh, G., and Jeney, V. (October, 2018) Influence of Iron on Bone Homeostasis. Pharmaceuticals, 11(4).

[71] Puri, N., Sodhi, K., Haarstad, M., Kim, D. H., Bohinc, S., Foglio, E., Favero, G., and Abraham, N. G. (June, 2012) Heme Induced Oxidative Stress Attenuates Sirtuin1 and Enhances Adipogenesis in Mesenchymal Stem Cells and Mouse Pre-Adipocytes. Journal of Cellular Biochemistry, 113(6), 1926-1935.

[72] Luo, Y., Tao, H., Jin, L., Xiang, W., and Guo, W. (November, 2019) CDKN2B-AS1 Exerts Oncogenic Role in Osteosarcoma by Promoting Cell Proliferation and Epithelial to Mesenchymal Transition. Cancer Biotherapy and Radiopharmaceuticals,.

[73] Congrains, A., Kamide, K., Ohishi, M., and Rakugi, H. (January, 2013) ANRIL: Molecular Mechanisms and Implications in Human Health. International Journal of Molecular Sciences, 14(1), 1278-1292.

[74] Yin, Z., Ding, H., He, E., Chen, J., and Li, M. (October, 2016) Overexpression of long non-coding RNA MFI2 promotes cell proliferation and suppresses apoptosis in human osteosarcoma. Oncology Reports, 36(4), 2033-2040.

[75] Li, C., Tan, F., Pei, Q., Zhou, Z., Zhou, Y., Zhang, L., Wang, D., and Pei, H. (2019) Noncoding RNA MFI2-AS1 promotes colorectal cancer cell proliferation, migration and invasion through miR-574-5p/MYCBP axis. Cell Proliferation, 52(4), e12632. 
[76] Zhu, C., Huang, L., Xu, F., Li, P., Li, P., and Hu, F. (October, 2019) LncRNA PCAT6 promotes tumor progression in osteosarcoma via activation of TGF- pathway by sponging miR-185-5p. Biochemical and Biophysical Research Communications,.

[77] Dong, P., Xiong, Y., Yue, J., Hanley, S. J. B., Kobayashi, N., Todo, Y., and Watari, H. (October, 2018) Long Non-coding RNA NEAT1: A Novel Target for Diagnosis and Therapy in Human Tumors. Frontiers in Genetics, 9.

[78] Ahmed, A. S. I., Dong, K., Liu, J., Wen, T., Yu, L., Xu, F., Kang, X., Osman, I., Hu, G., Bunting, K. M., Crethers, D., Gao, H., Zhang, W., Liu, Y., Wen, K., Agarwal, G., Hirose, T., Nakagawa, S., Vazdarjanova, A., and Zhou, J. (September, 2018) Long noncoding RNA NEAT1 (nuclear paraspeckle assembly transcript 1) is critical for phenotypic switching of vascular smooth muscle cells. Proceedings of the National Academy of Sciences, 115(37), E8660-E8667.

[79] Taiana, E., Favasuli, V., Ronchetti, D., Todoerti, K., Pelizzoni, F., Manzoni, M., Barbieri, M., Fabris, S., Silvestris, I., Cantafio, M. E. G., Platonova, N., Zuccal, V., Maltese, L., Soncini, D., Ruberti, S., Cea, M., Chiaramonte, R., Amodio, N., Tassone, P., Agnelli, L., and Neri, A. (August, 2019) Long non-coding RNA NEAT1 targeting impairs the DNA repair machinery and triggers anti-tumor activity in multiple myeloma. Leukemia, pp. 1-11.

[80] Wan, G., Mathur, R., Hu, X., Liu, Y., Zhang, X., Peng, G., and Lu, X. (May, 2013) Long non-coding RNA ANRIL (CDKN2B-AS) is induced by the ATM-E2F1 signaling pathway. Cellular signalling, 25(5), 1086-1095.

[81] Ding, K., Liao, Y., Gong, D., Zhao, X., and Ji, W. (July, 2018) Effect of long non-coding RNA H19 on oxidative stress and chemotherapy resistance of CD133+ cancer stem cells via the MAPK/ERK signaling pathway in hepatocellular carcinoma. Biochemical and Biophysical Research Communications, 502(2), 194-201.

[82] Yu, J.-L., Li, C., Che, L.-H., Zhao, Y.-H., and Guo, Y.-B. (2019) Downregulation of long noncoding RNA H19 rescues hippocampal neurons from apoptosis and oxidative stress by inhibiting IGF2 methylation in mice with streptozotocin-induced diabetes mellitus. Journal of Cellular Physiology, 234(7), 10655-10670.

[83] Hazell, G. G. J., Peachey, A. M. G., Teasdale, J. E., Sala-Newby, G. B., Angelini, G. D., Newby, A. C., and White, S. J. (December, 2016) PI16 is a shear stress and inflammationregulated inhibitor of MMP2. Scientific Reports, 6.

[84] Puvvula, P. K. (May, 2019) LncRNAs Regulatory Networks in Cellular Senescence. International Journal of Molecular Sciences, 20(11).

[85] Spanner, M., Weber, K., Lanske, B., Ihbe, A., Siggelkow, H., Schtze, H., and Atkinson, M. J. (August, 1995) The iron-binding protein ferritin is expressed in cells of the osteoblastic lineage in vitro and in vivo. Bone, 17(2), 161-165.

[86] Balogh, E., Tolnai, E., Nagy, B., Nagy, B., Balla, G., Balla, J., and Jeney, V. (September, 2016) Iron overload inhibits osteogenic commitment and differentiation of mesenchymal stem cells via the induction of ferritin. Biochimica et Biophysica Acta (BBA) - Molecular Basis of Disease, 1862(9), 1640-1649. 
[87] Zarjou, A., Jeney, V., Arosio, P., Poli, M., Antal-Szalms, P., Agarwal, A., Balla, G., and Balla, J. (June, 2009) Ferritin Prevents Calcification and Osteoblastic Differentiation of Vascular Smooth Muscle Cells. Journal of the American Society of Nephrology, 20(6), 12541263.

[88] Doi, M., Nagano, A., and Nakamura, Y. (January, 2002) Genome-wide Screening by cDNA Microarray of Genes Associated with Matrix Mineralization by Human Mesenchymal Stem Cells in Vitro. Biochemical and Biophysical Research Communications, 290(1), 381-390.

[89] Liu, Z., Zheng, Z., Qi, J., Wang, J., Zhou, Q., Hu, F., Liang, J., Li, C., Zhang, W., and Zhang, X. (December, 2018) CD24 identifies nucleus pulposus progenitors/notochordal cells for disc regeneration. Journal of Biological Engineering, 12(1), 35.

[90] Tsai, Y.-H., Lin, K.-L., Huang, Y.-P., Hsu, Y.-C., Chen, C.-H., Chen, Y., Sie, M.-H., Wang, G.-J., and Lee, M.-J. (2015) Suppression of ornithine decarboxylase promotes osteogenic differentiation of human bone marrow-derived mesenchymal stem cells. FEBS Letters, 589(16), 2058-2065.

[91] Chang, C.-F., Hsu, K.-H., Shen, C.-N., Li, C.-L., and Lu, J. (2014) Enrichment and Characterization of Two Subgroups of Committed Osteogenic Cells in the Mouse Endosteal Bone Marrow with Expression Levels of CD24. Journal of Bone Research, 2(2), 1-9.

[92] Park, G. C., Song, J. S., Park, H.-Y., Shin, S.-C., Jang, J. Y., Lee, J.-C., Wang, S.-G., Lee, B.-J., and Jung, J.-S. (May, 2016) Role of Fibroblast Growth Factor-5 on the Proliferation of Human Tonsil-Derived Mesenchymal Stem Cells. Stem Cells and Development, 25(15), 1149-1160.

[93] Kornmann, M., Ishiwata, T., Beger, H. G., and Korc, M. (September, 1997) Fibroblast growth factor-5 stimulates mitogenic signaling and is overexpressed in human pancreatic cancer: evidence for autocrine and paracrine actions. Oncogene, 15(12), 1417-1424.

[94] Williamson, E. A., Wray, J. W., Bansal, P., and Hromas, R. (2012) Overview for the Histone Codes for DNA Repair. Progress in molecular biology and translational science, 110, 207227.

[95] Wang, S., Hu, B., Ding, Z., Dang, Y., Wu, J., Li, D., Liu, X., Xiao, B., Zhang, W., Ren, R., Lei, J., Hu, H., Chen, C., Chan, P., Li, D., Qu, J., Tang, F., and Liu, G.-H. (January, 2018) ATF6 safeguards organelle homeostasis and cellular aging in human mesenchymal stem cells. Cell Discovery, 4.

[96] Fu, L., Hu, Y., Song, M., Liu, Z., Zhang, W., Yu, F.-X., Wu, J., Wang, S., Izpisua Belmonte, J. C., Chan, P., Qu, J., Tang, F., and Liu, G.-H. (April, 2019) Up-regulation of FOXD1 by YAP alleviates senescence and osteoarthritis. PLoS Biology, 17(4).

[97] Samsonraj, R. M., Dudakovic, A., Manzar, B., Sen, B., Dietz, A. B., Cool, S. M., Rubin, J., and van Wijnen, A. J. (December, 2017) Osteogenic Stimulation of Human AdiposeDerived Mesenchymal Stem Cells Using a Fungal Metabolite That Suppresses the Polycomb Group Protein EZH2. Stem Cells Translational Medicine, 7(2), 197-209. 
[98] Dudakovic, A., Gluscevic, M., Paradise, C. R., Dudakovic, H., Khani, F., Thaler, R., Ahmed, F. S., Li, X., Dietz, A. B., Stein, G. S., Montecino, M. A., Deyle, D. R., Westendorf, J. J., and van Wijnen, A. J. (April, 2017) Profiling of human epigenetic regulators using a semiautomated real-time qPCR platform validated by next generation sequencing. Gene, 609, $28-37$.

[99] Camilleri, E. T., Gustafson, M. P., Dudakovic, A., Riester, S. M., Garces, C. G., Paradise, C. R., Takai, H., Karperien, M., Cool, S., Sampen, H.-J. I., Larson, A. N., Qu, W., Smith, J., Dietz, A. B., and van Wijnen, A. J. (August, 2016) Identification and validation of multiple cell surface markers of clinical-grade adipose-derived mesenchymal stromal cells as novel release criteria for good manufacturing practice-compliant production. Stem Cell Research $\&$ Therapy, 7.

[100] Knight, C., James, S., Kuntin, D., Fox, J., Newling, K., Hollings, S., Pennock, R., and Genever, P. (January, 2019) Epidermal growth factor can signal via -catenin to control proliferation of mesenchymal stem cells independently of canonical Wnt signalling. Cellular Signalling, 53, 256-268.

[101] Jiang, S., Cheng, S.-J., Ren, L.-C., Wang, Q., Kang, Y.-J., Ding, Y., Hou, M., Yang, X.-X., Lin, Y., Liang, N., and Gao, G. (September, 2019) An expanded landscape of human long noncoding RNA. Nucleic Acids Research, 47(15), 7842-7856.

[102] Chang, T.-H., Huang, H.-D., Ong, W.-K., Fu, Y.-J., Lee, O. K., Chien, S., and Ho, J. H. (April, 2014) The effects of actin cytoskeleton perturbation on keratin intermediate filament formation in mesenchymal stem/stromal cells. Biomaterials, 35(13), 3934-3944.

[103] Chang, Y., Li, H., and Guo, Z. (2014) Mesenchymal stem cell-like properties in fibroblasts. Cellular Physiology and Biochemistry: International Journal of Experimental Cellular Physiology, Biochemistry, and Pharmacology, 34(3), 703-714.

[104] Denu, R. A., Nemcek, S., Bloom, D. D., Goodrich, A. D., Kim, J., Mosher, D. F., and Hematti, P. (August, 2016) Fibroblasts and Mesenchymal Stromal/Stem Cells Are Phenotypically Indistinguishable. Acta Haematologica, 136(2), 85-97.

[105] Ball, S. G., Shuttleworth, A. C., and Kielty, C. M. (April, 2004) Direct cell contact influences bone marrow mesenchymal stem cell fate. The International Journal of Biochemistry \& Cell Biology, 36(4), 714-727.

[106] Tamama, K., Sen, C. K., and Wells, A. (October, 2008) Differentiation of Bone Marrow Mesenchymal Stem Cells into the Smooth Muscle Lineage by Blocking ERK/MAPK Signaling Pathway. Stem Cells and Development, 17(5), 897-908.

[107] Kumar, A., DSouza, S. S., Moskvin, O. V., Toh, H., Wang, B., Zhang, J., Swanson, S., Guo, L.-W., Thomson, J. A., and Slukvin, I. I. (May, 2017) Specification and Diversification of Pericytes and Smooth Muscle Cells from Mesenchymoangioblasts. Cell Reports, 19(9), 1902-1916. 


\section{$5 \quad$ Figure Legends}

\subsection{Figure 1: Flowchart representation of the Pipeline used for this in the study.}

The 4 steps of the flowchart are described A) Ab initio reconstruction of transcrit expressed in MSC from SRA dataset and creation of a reference (gtf+fasta) for quantification of Ensembl annotated genes, unannotated intergenic (Mlincs) and unannotated overlapping antisens (Mloanc). The results are shown in Fig2.

B) Differential Analysis for the selection of MSC markers (restrained candidate set) with i/ kallisto pseudoalignement and Sleuth differential test followed by feature selection by random forest with Boruta package. Long read sequencing and active transcription in MSC by epigenetic marks information completed the selection step (see figures 2 and 3).

C) Validation of cell expression specificity of candidates by kmer quantification in ENCODE RNAseq datasets ( see table S2 for list of data) and qPCR validation. The results are presented in figure 4.

D) Functional investigations were performed with in silico prediction methods from the sequence of candidates, followed by k-mer quantification with FANTOM6 data set, single cell RNAseq and selected MSC conditions. Kmer quantification phases are shown by corresponding icons (figures 5 and 6).

\subsection{Figure 2: Overview of annotated genes and unannotated tran- scripts enriched in BM-MSC.}

A) Left pannels represented: i/EnsemblV90 transcript categories and distribution, ii/ transcripts distribution expressed in MSCs, showing unnatotated transcripts obtained with Ab initio reconstruction by StringTie vs annotated transcripts (expression $>0.1 \mathrm{TPM}$ ) iii/ Predicted long non-coding RNA(lncRNA) from unnanotated reconstructed transcripts include new long non coding RNA with intergenic (Mlinc) and antisens (Mlncoa) RNA categories.

B-C-D) Distribution of transcript length, exon length and GC percentage across different categories respectively with the same colors as in A pannel : coding transcripts (blue), annotated lincRNA (pink), annotated overlapping antisens lncRNA (purple), novel lincRNA (Mlincs, yellow), novel overlapping antisens RNA (Mloanc, red).

E) Representation of annotated genes (top pannel) and unannotated transcripts (bottom pannel) overexpresed in MSC versus non-MSC types $(\log 2 \mathrm{FC}>0.5$ and padj $<0.05)$, separately showed in MA plot.

F) Total number of transcripts transcript by category. The colored bar indicated the number of differential expression of annotated genes (Ensemblv90) and unannotated transcripts (Mlinc and Mloanc).

G) Global expression in BM-MSC (with Sleuth normalization) of the same categories as in F for annotated genes and unannotated transcripts. 


\subsection{Figure 3: Selection of a refined set of best candidates by random forest (top35), long read sequencing and epigenetic features.}

A) Expression of the best MSC-specific candidates selected by boruta machine learning along MSC group and not MSC cohorts. Left pannel : top35 most relevant annotated genes (non-coding included); Right pannel: unannotated intergenic lncRNAs (Mlincs) and their average importance scores determined by Boruta method displayed in upside line plot.

B) Genomic visualisation of Mlincs predictions 28428, 128022, 89912, and 64225 (MSClinc orange) from short reads alignement of all MSC group files (blue/magenta and bam visualisation), compared with long read alignements (long reads grey). Additional epigenomic features are shown to reveal active transcriptional activity from trimethylation of Histone H3 (H3K4me3), acetylation of Histone H3 H3K27 in MSCs (H3K4me3 and H3K27ac, green), and Dnase sensibitity hotspots of MSC (MSC DNAse, red).

\subsection{Figure 4: High throughput exploration of selected candidate across strong variety of samples by k-mer quantification in RNAseq and biological validation by RT-qPCR.}

A : List of tissues for the cell specific expression exploration (samples with ID numbers are listed in table $\mathrm{S} 1$ )

B: Relative expression of Mlinc.28428.2, Mlinc.128022.2, and Mlinc.89912.1 across ENCODEs ribodepleted RNAseq datas, made by k-mer quantification, normalized by k-mer by million.

$\mathrm{C}$ : qPCR relative quantification was performed on the selected 3 Mlincs in MSC of different origins (BM-MSC, Ad-MSC, Umbilical cord msc) and other indicated cell types. Relative quantification (Log induction) was quantified by ddCt method using non MSC types types as calibrator (mean of triplicates). Student tests have been made between triplicates, each test use BM-MSCs as reference group (ns: $\mathrm{P}>0.05, *: \mathrm{P} \leq 0.05$, **: $\mathrm{P} \leq 0.01$, ***: $\mathrm{P} \leq 0.001, * * * *: \mathrm{P} \leq 0.0001$ ).

\subsection{Figure 5: Prediction of potential functions of candidates with k- mer quantification and single-cell.}

For each Mlinc (Mlinc.28428 (A), Mlinc.128022 (B) and Mlinc.89912 (C) respectively) 3 steps of prediction were performed. a. Enrichment in the different subcompartments of fibroblasts from FANTOM6 dataset: Free nuclear fraction (Nuc), chromatin (Chr) and cytoplasm (Cyt); b. Expression of marker in FANTOM6 data depending of the KnockDown (KD) of an annotated lncRNA. Normalised count of all specific k-mers is averaged by sample (zeros vlues deleted) and t-tests are made between control and KD fibroblasts. c. General expression of Mlincs inside AdMSC population, dimensionnal reduction made with UMAP method, made from batch corrected counts. Expression of differentially expressed annotated genes between positive and negative cells for Mlinc.28428, Mlinc.128022 and Mlinc.89912 respectively. 


\subsection{Figure 6:Expression of markers in different datasets from SRA in cell conditions related to previous findings}

A) Expression of Mlinc.28428.1 in the context of oxydative, replicative, or KO-driven, stress and senescence (PRJNA396193, PRJNA433339). Relevant changes of expressions are showed with t-test results (ns: $\mathrm{P}>0.05, *: \mathrm{P} \leq 0.05, * *: \mathrm{P} \leq 0.01, * * *: \mathrm{P} \leq 0.001, * * * *: \mathrm{P} \leq 0.0001$ ).

B) Expression of Mlinc.128022 in osteodifferentiation conditions (PRJNA515466) or osteodifferentiation potential (PRJNA379707). Relevant changes of expressions are showed with t-test results (ns: $\mathrm{P}>0.05, *: \mathrm{P} \leq 0.05, * *: \mathrm{P} \leq 0.01, * * *: \mathrm{P} \leq 0.001, * * * *: \mathrm{P} \leq 0.0001)$.

C)Expression of Mlinc.89912 in the context of proliferation (PRJNA328824 and PRJNA498109). Relevant changes of expressions are showed with t-test results (ns : $\mathrm{P}>0.05,{ }^{*}: \mathrm{P} \leq 0.05$, **: $\mathrm{P}$ $\leq 0.01, * * *: \mathrm{P} \leq 0.001, * * * *: \mathrm{P} \leq 0.0001)$. The detailed list of datasets is provided in table S4. 


\section{Figure 1}

bioRxiv preprint doi: https://doi.org/10.1101/2020.03.09.976001; this version posted March 11, 2020. The copyright holder for this preprint (which was not certified by peer review) is the author/funder, who has granted bioRxiv a license to display the preprint in perpetuity. It is made available under aCC-BY-ND 4.0 International license.

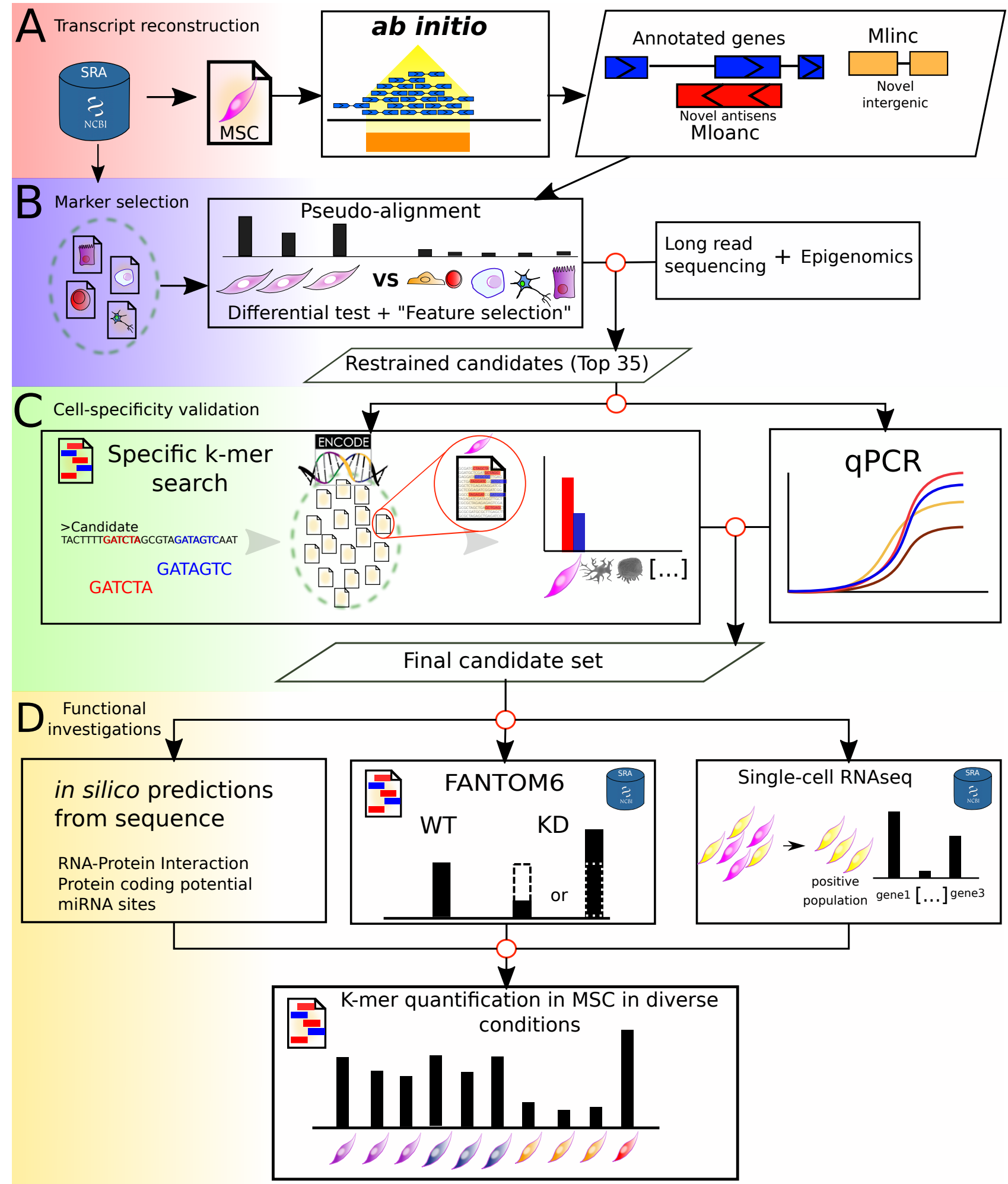




\section{Figure 2}

bioRxiv preprint doi: https://doi.org/10.1101/2020.03.09.976001; this version posted March 11, 2020. The copyright holder for this preprint (which was not certified by peer review) is the author/funder, who has granted bioRxiv a license to display the preprint in perpetuity. It is made available under aCC-BY-ND 4.0 International license.
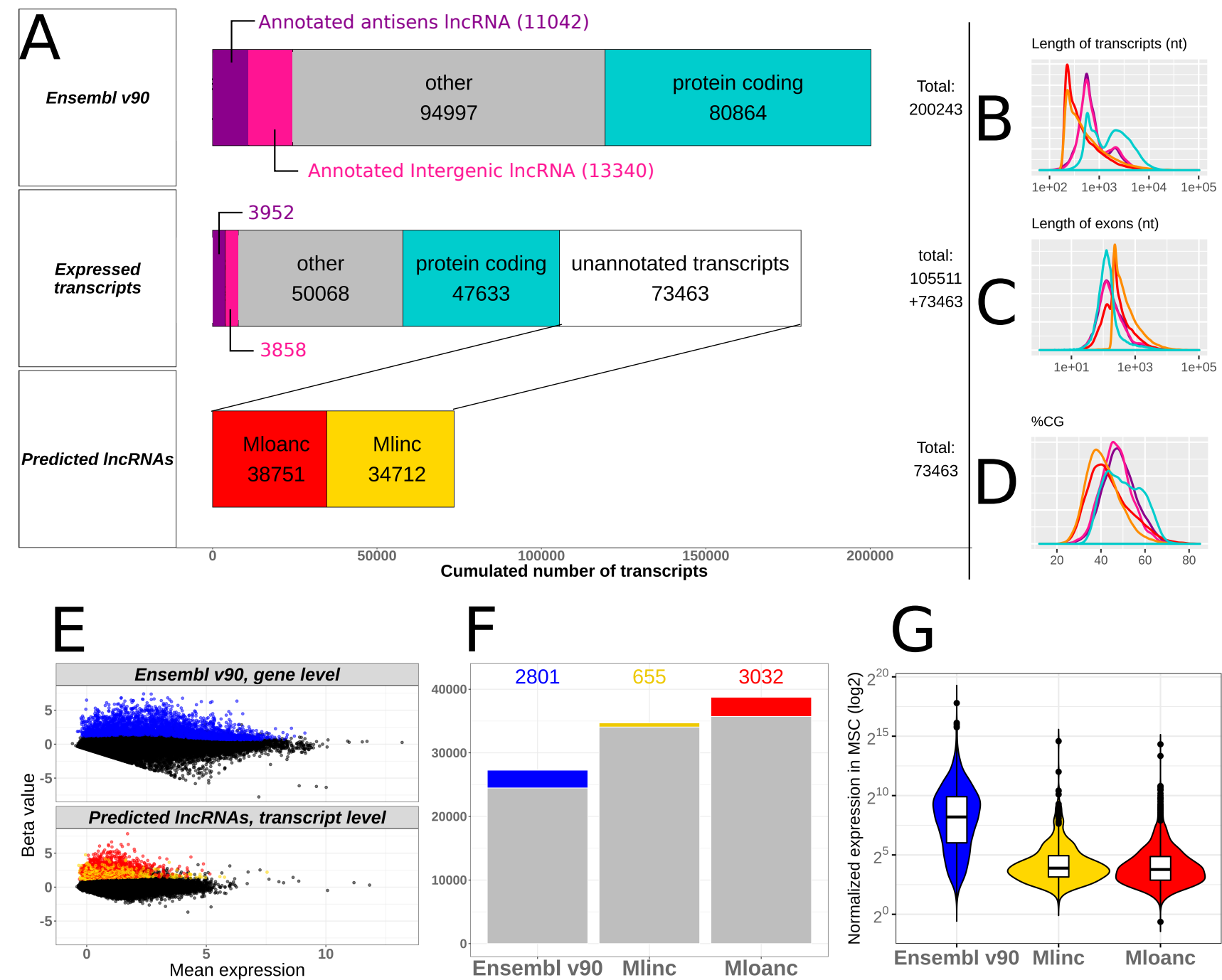

G

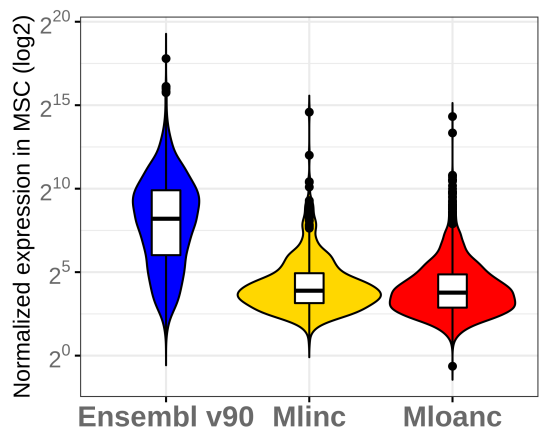




\section{Figure 3}

bioRxiv preprint doi: https://doi.org/10.1101/2020.03.09.976001; this version posted March 11, 2020. The copyright holder for this preprint (which was not certified by peer review) is the author/funder, who has granted bioRxiv a license to display the preprint in perpetuity. It is made

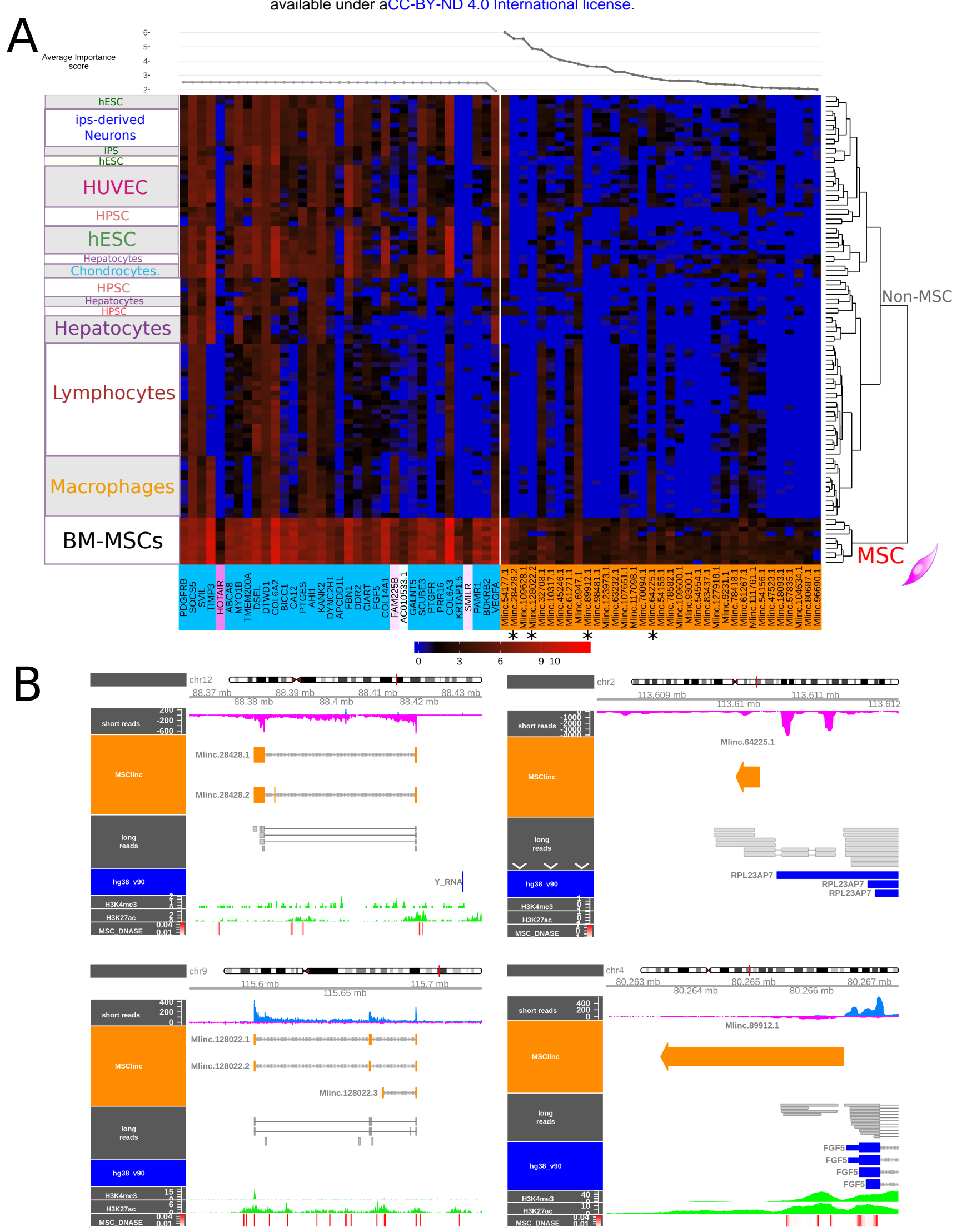




\section{Figure 4}

bioRxiv preprint doi: https://doi.org/10.1101/2020.03.09.976001; this version posted March 11, 2020. The copyright holder for this preprint (A hich was not certified by peer (eyjew) is the author/funder, who has granted bioRxiv a IIcense to display the preprint in perpetuity. It is made

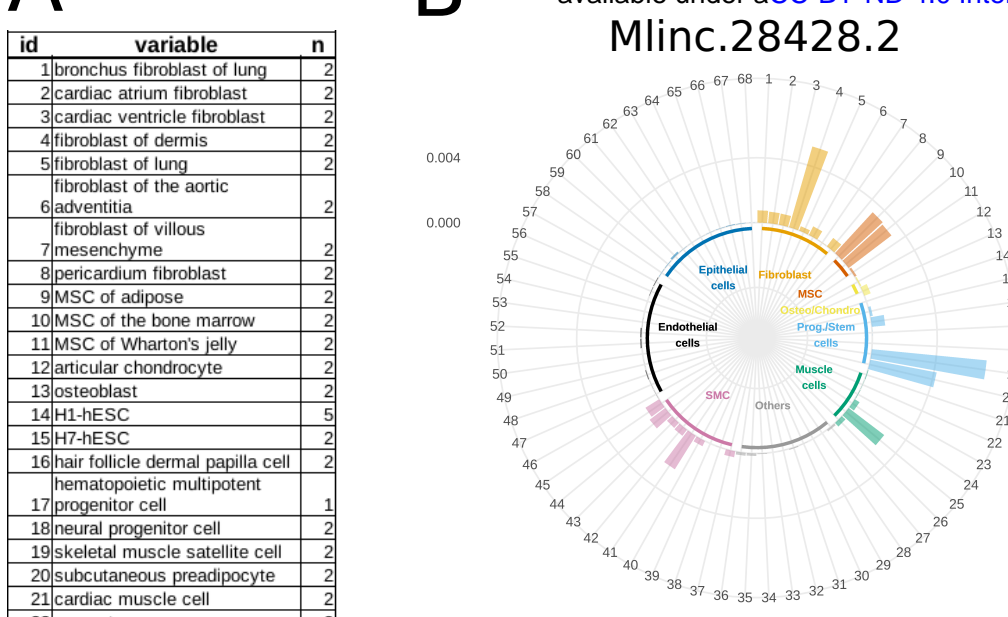

uns preadipocyt

21 cardiac muscle ced

23 myometrial cell

\begin{tabular}{l|l}
24 & myotube \\
25 & regular cardiac myocyte
\end{tabular}

26 skeletal muscle myoblast

27. astrocyte

28 bipolar neuron

30 foreskin keratinocyte

31 hair follicular keratinocyte

32 hepatocyte

33 melanocyte of skin

34 mesangial cell

36 placental pericyte

37 aortic SMC

38 bronchial smooth muscle cell

39 smooth muscle cell

40 smooth muscle cell of bladder

41 coronary artery

smooth muscle cell of the

pulmonary artery

43 umbilical artery

44 smooth muscle cell of trachea

45 uterine smooth muscle cell

46 endothelial cell

dermis blood ves

endothelial cell

dermis lymphatic vesse

ndothelial cell

lymphatic vessel endothel

endometrial microvascular

endothelial cells

endothelial cell of coronary

artery

glomerular endothelial ce ung microvascular endotheli

mammary microvascular

endothelial cel

pulmonary artery endothelial

thoracic aorta endothelial cell

57 vein endothelial cell

59 airway epithelial cell

epithelial cell of alveolus of

epithelial cell of alveolus o

ung

pithelial cell of proximal

tubule

epithelial cell of umbilical

2 artery

3 kidney epithelial cell

4 mammary epithelial cell

epithelial cell of viscerocran

5 mucosa

66 placental epithelial cell

67 renal cortical epithelial $c$

68 tracheal epithelial cell

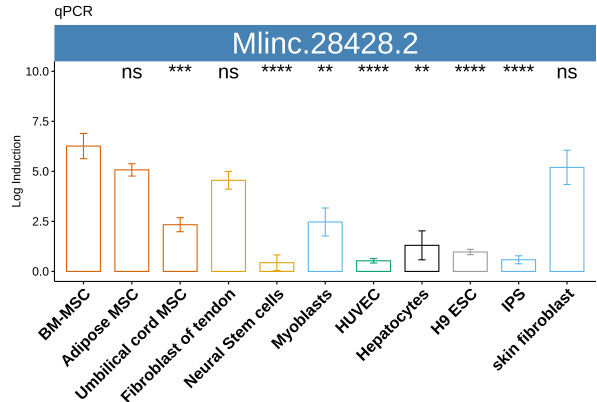

MIinc. 28428.2

\section{Mlinc 28428.2}

\section{Mlinc. 128022.2}
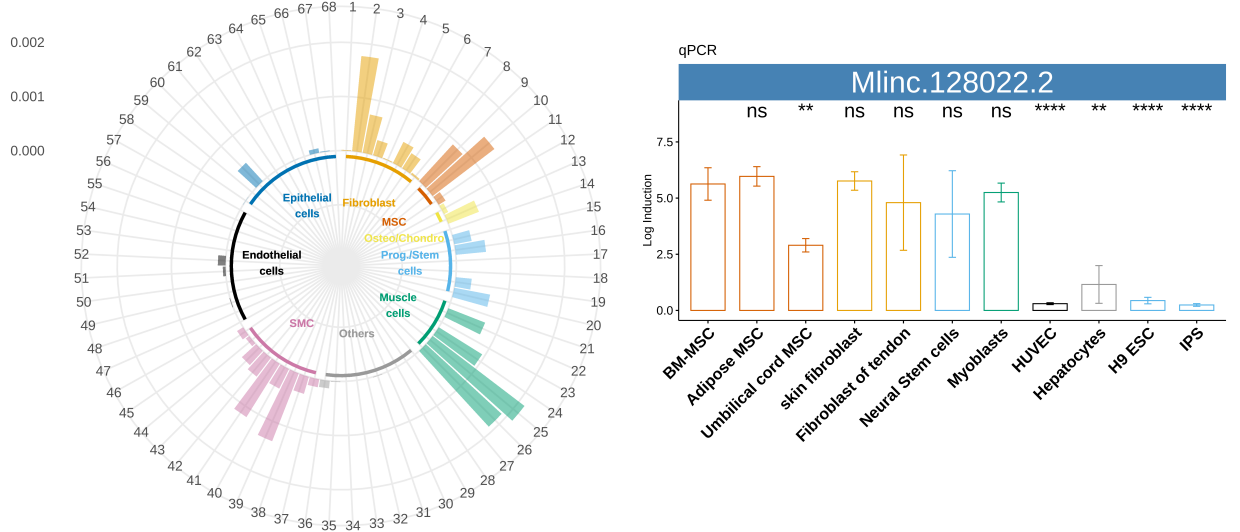

Mlinc.89912.1
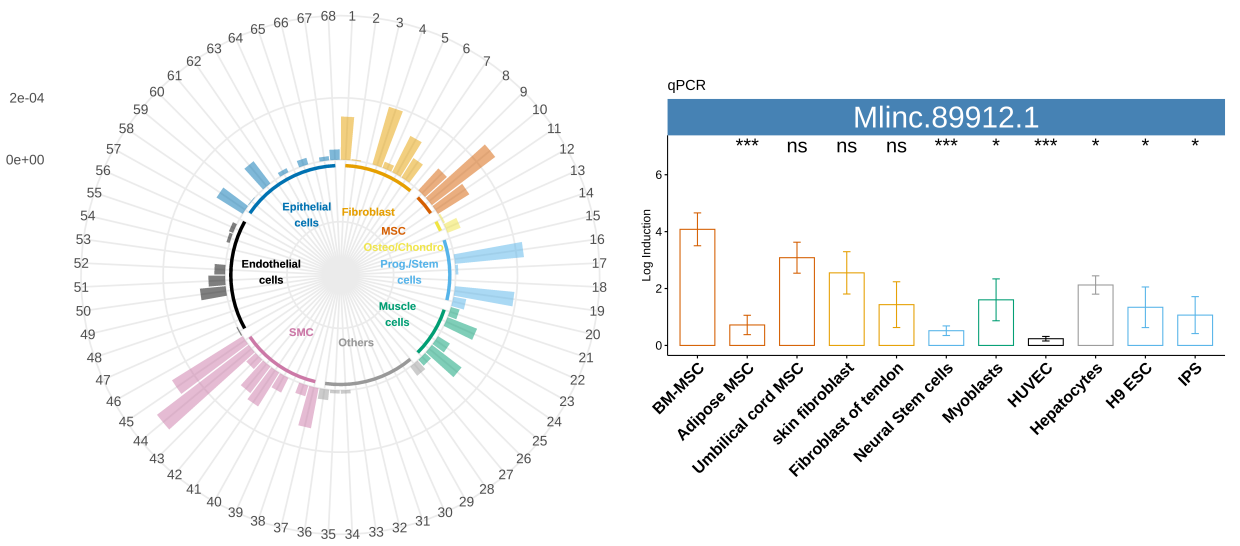


\section{Figure 5}

bioRxiv preprint doi: https://doi.org/10.1101/2020.03.09.976001; this version posted March 11, 2020. The copyright holder for this preprint (which was not certified by peer review) is the author/funder, who has granted bioRxiv a license to display the preprint in perpetuity. It is made available under aCC-BY-ND 4.0 International license.

\section{A: Mlinc. 28428}

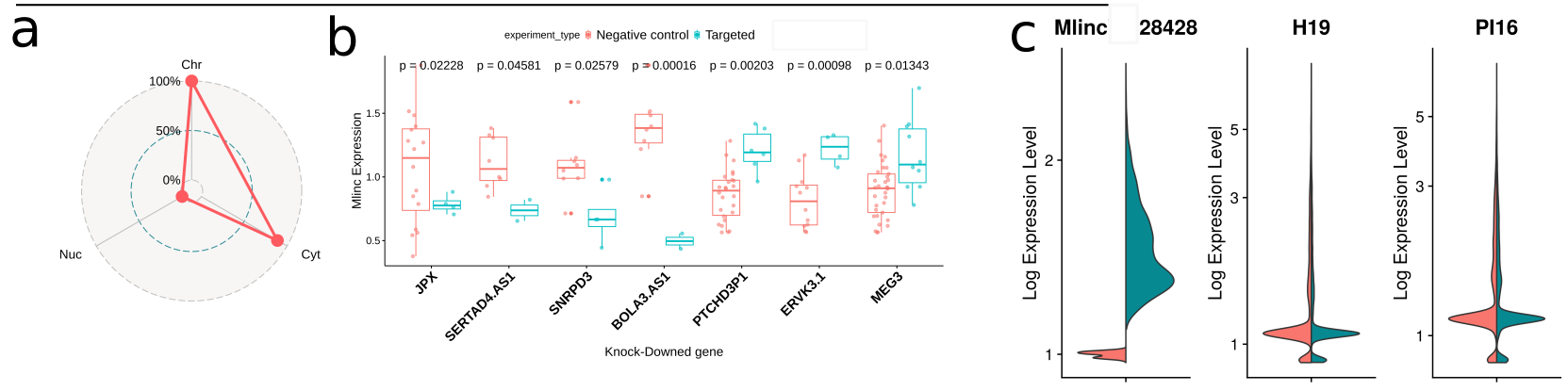

\section{B: Mlinc. 128022}

a

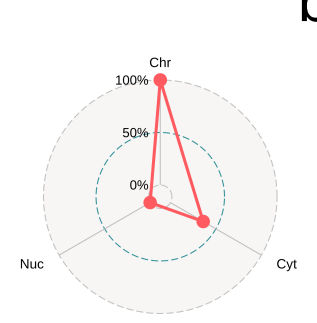

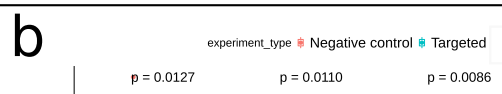

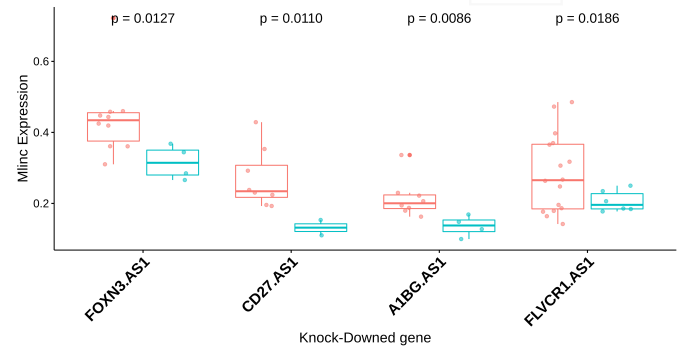

Knock-Downed gene
C

焉 Mlinc' 128022

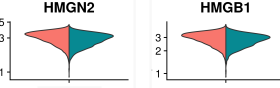

ODC1
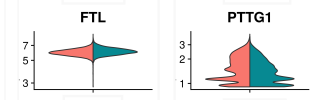

BIRC5
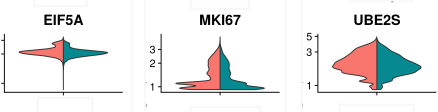

FGF5

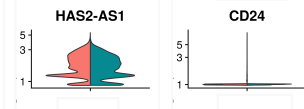

\section{C : Mlinc.89912}

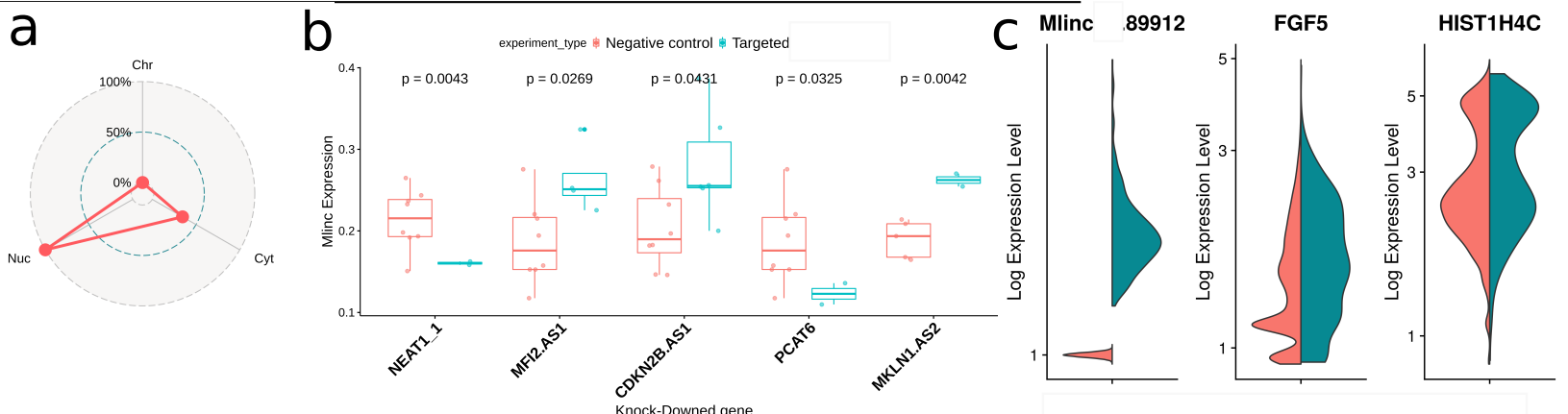




\section{Figure 6}

bioRxiv preprint doi: https://doi.org/10.1101/2020.03.09.976001; this version posted March 11, 2020. The copyright holder for this preprint (which was not certified by peer review) is the author/funder, who has granted bioRxiv a license to display the preprint in perpetuity. It is made

\section{A: Mlinc. 28428}

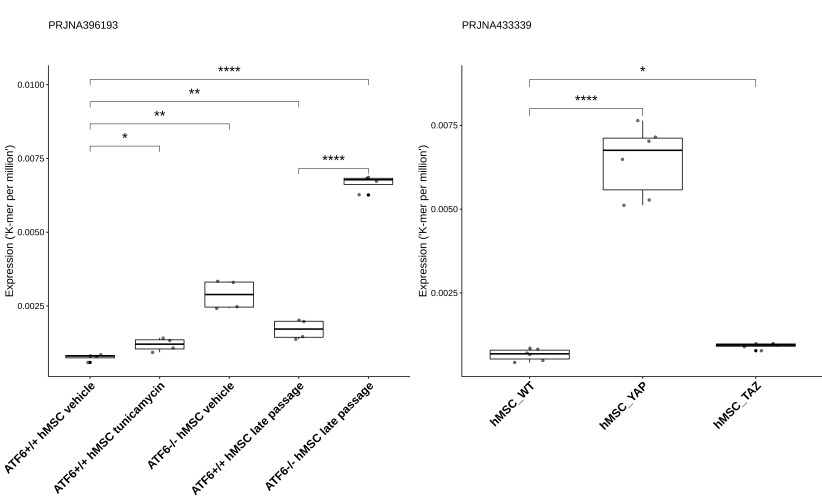

\section{B: Mlinc. 128022}
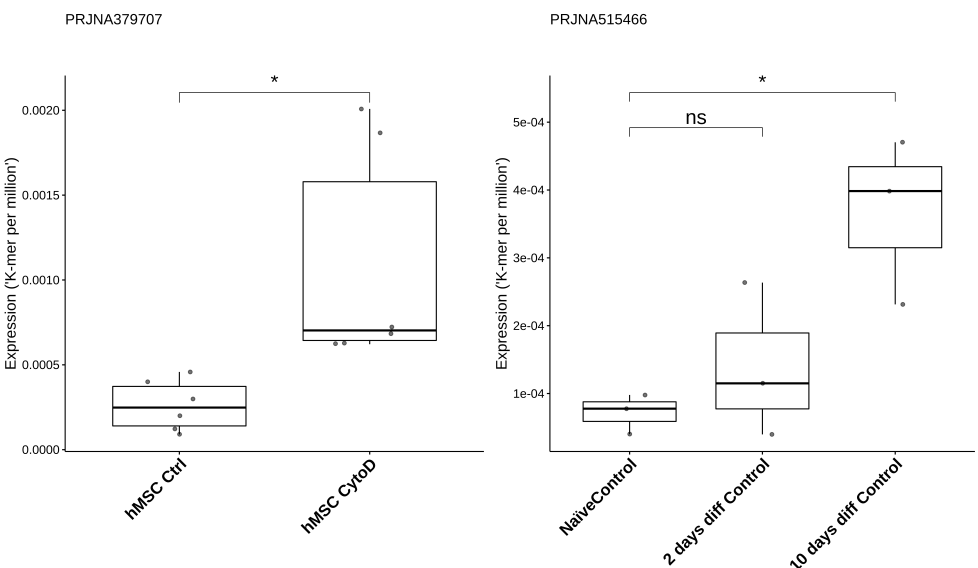

\section{C : Mlinc. 89912}

PRJNA328824

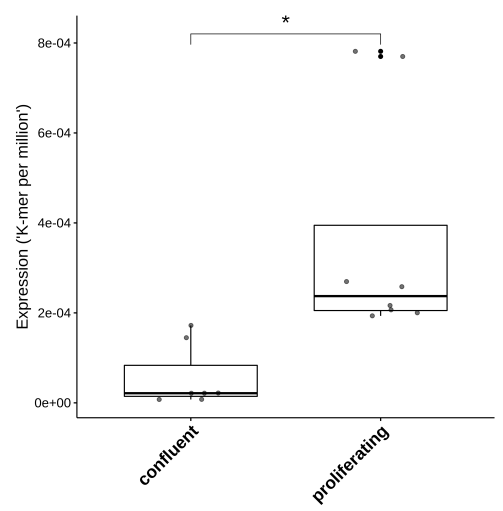

PRJNA498109

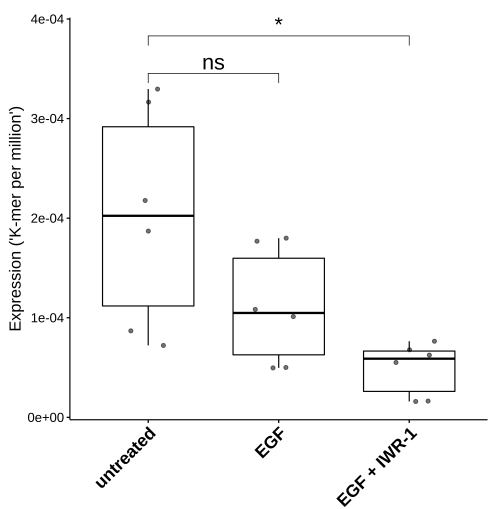


bioRxiv preprint doi: https://doi.org/10.1101/2020.03.09.976001; this version posted March 11, 2020. The copyright holder for this preprint (which was not certified by peer review) is the author/funder, who has granted bioRxiv a license to display the preprint in perpetuity. It is made available under aCC-BY-ND 4.0 International license.

\section{Supplementary figures}

sebastien.riquier

January 2020 
bioRxiv preprint doi: https://doi.org/10.1101/2020 03.09 .976001 ; this version posted March 11, 2020. The copyright holder for this preprint (which was not certified by peer review) is the author/funder, who has granted bioRxiv a license to display the preprint in perpetuity. It is made available under aCC-BY-ND 4.0 International license.

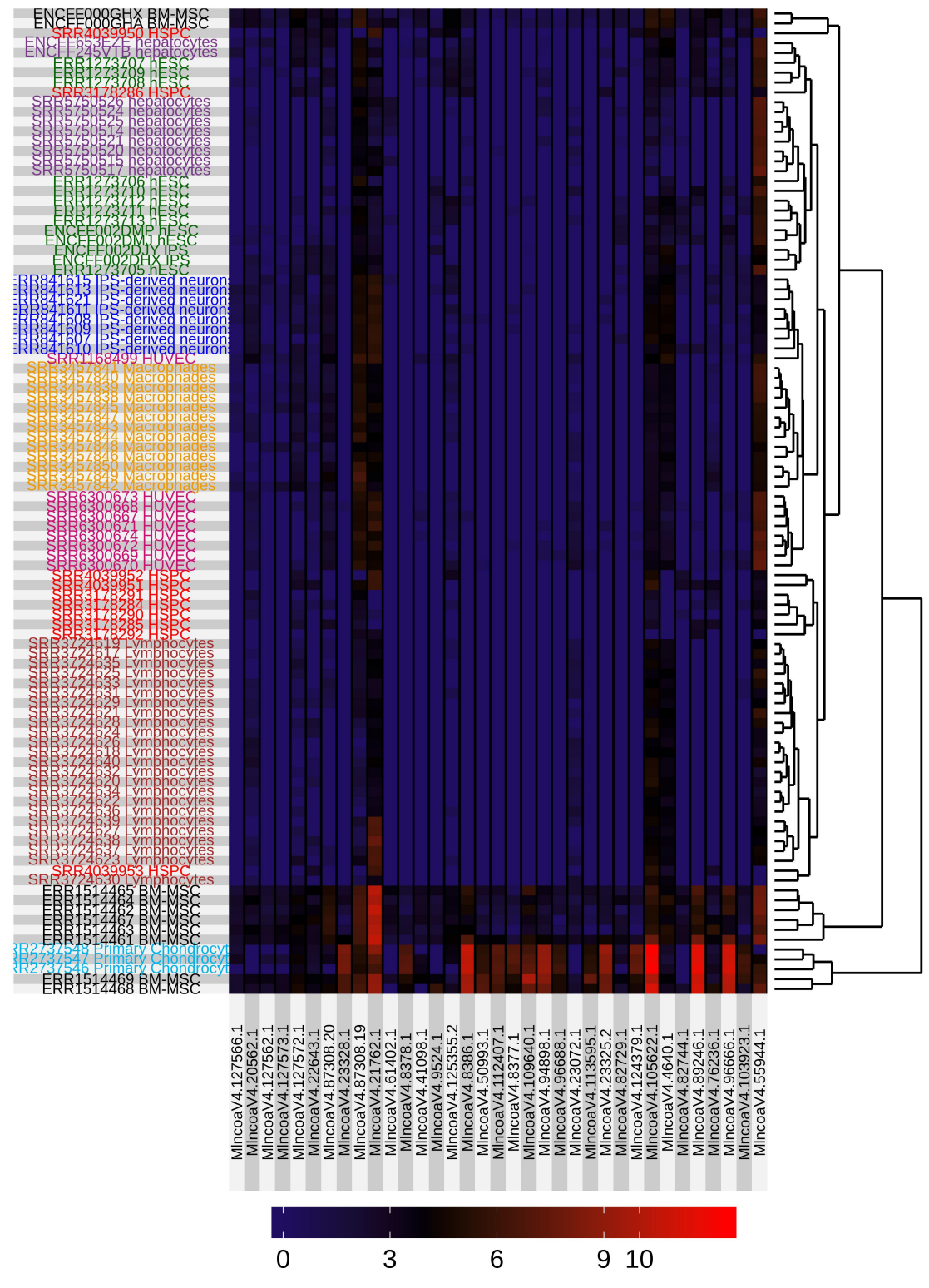

Supplementary Figure 1: Expression of Mloanc (Antisens unannotated RNA) selected after feature selection in the differential analysis cohort 


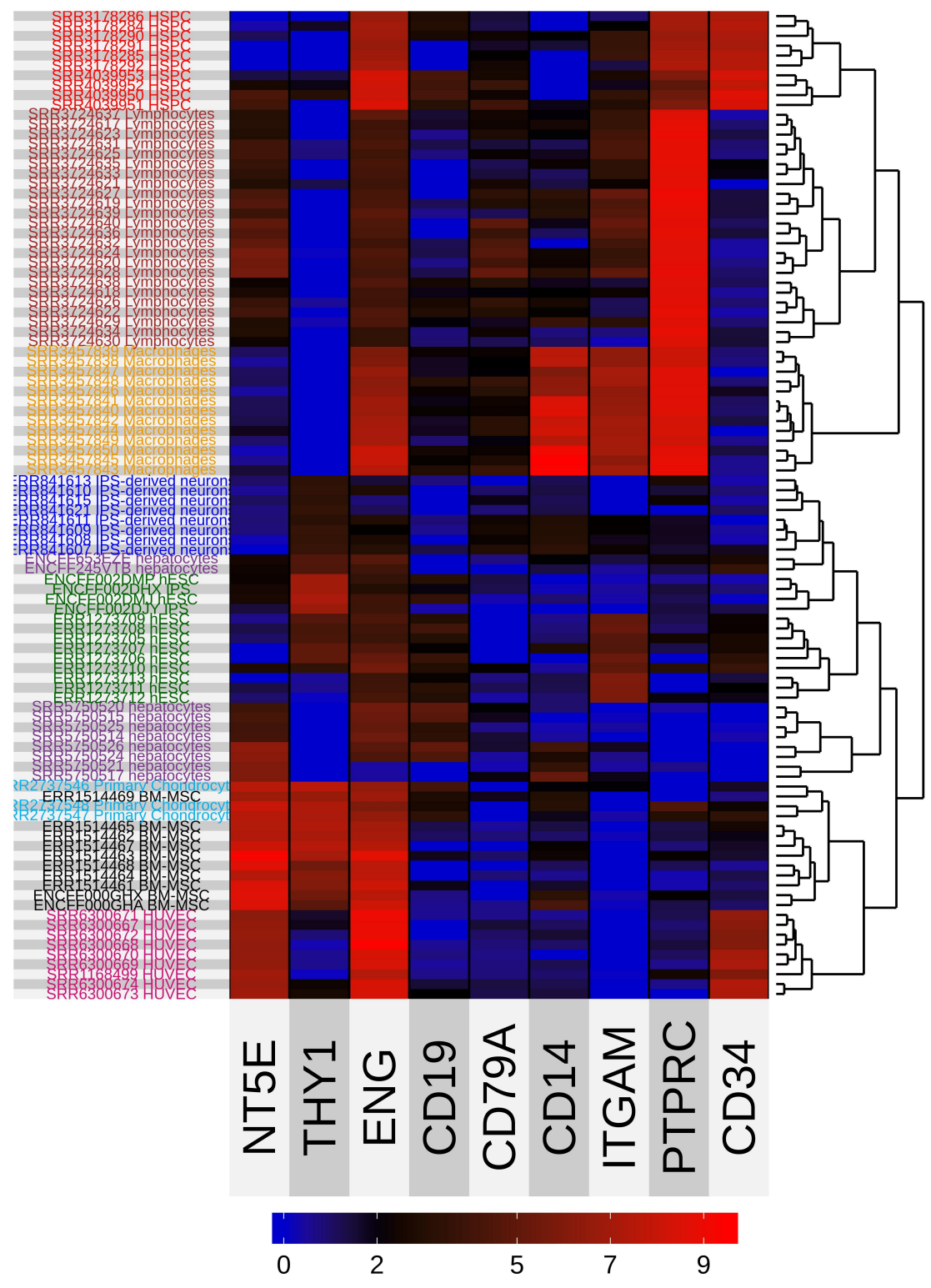

Supplementary Figure 2: Expression of ISCT's MSC markers in the differential analysis cohort THY1 $=$ CD90, NT5E $=$ CD73, ENG $=$ CD105, $\mathrm{ITGAM}=\mathrm{CD} 11 \mathrm{~B}, \mathrm{PTPRC}=\mathrm{CD} 45$ 
bioRxiv preprint doi: https://doi.org/10.1101/2020 03.09 .976001 ; this version posted March 11, 2020. The copyright holder for this preprint (which was not certified by peer review) is the author/funder, who has granted bioRxiv a license to display the preprint in perpetuity. It is made available under aCC-BY-ND 4.0 International license.

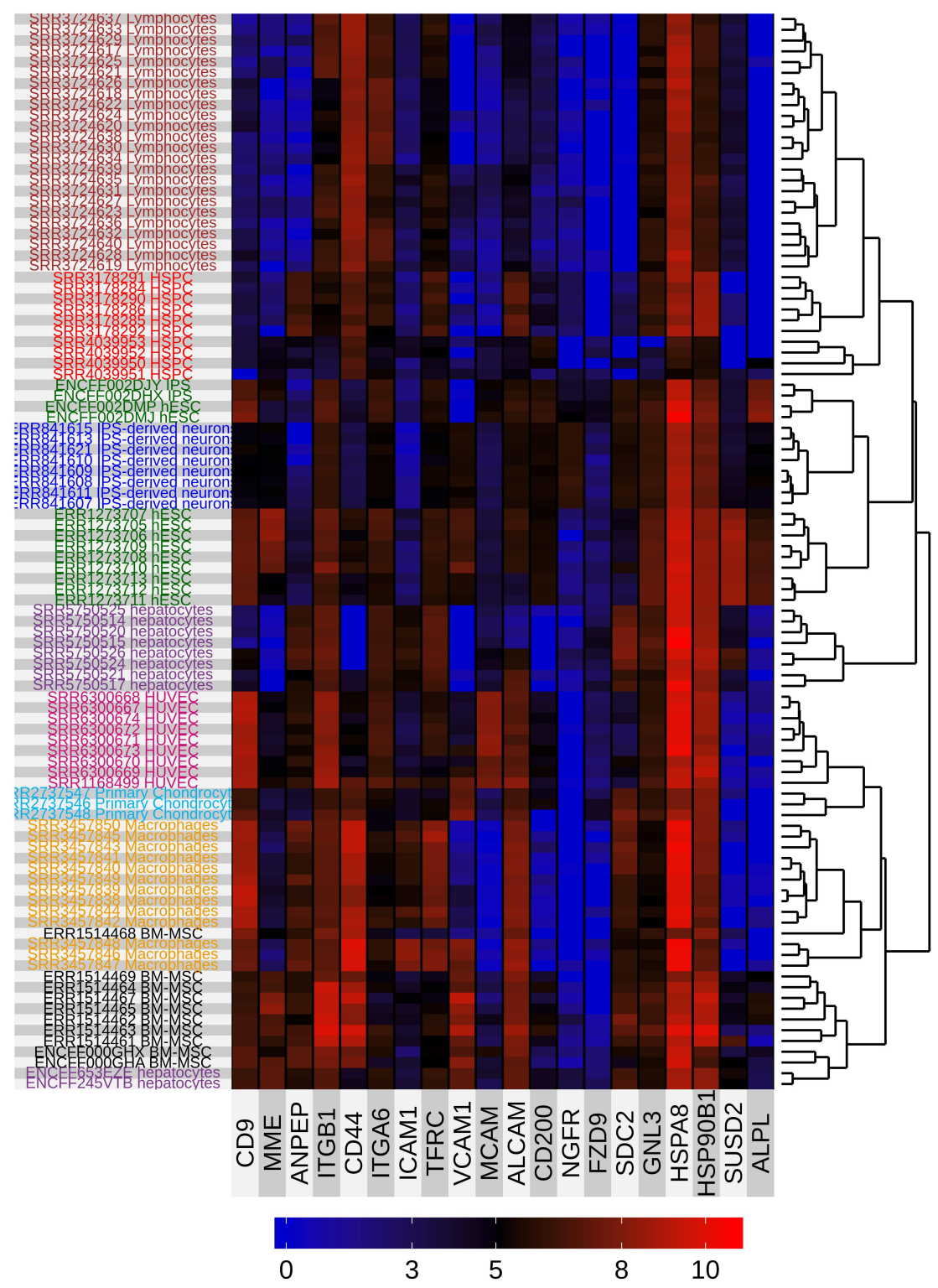

Supplementary Figure 3: Heatmap present positive markers for MSC proposed in the bibliography 


\section{ENG-ENST00000344849}

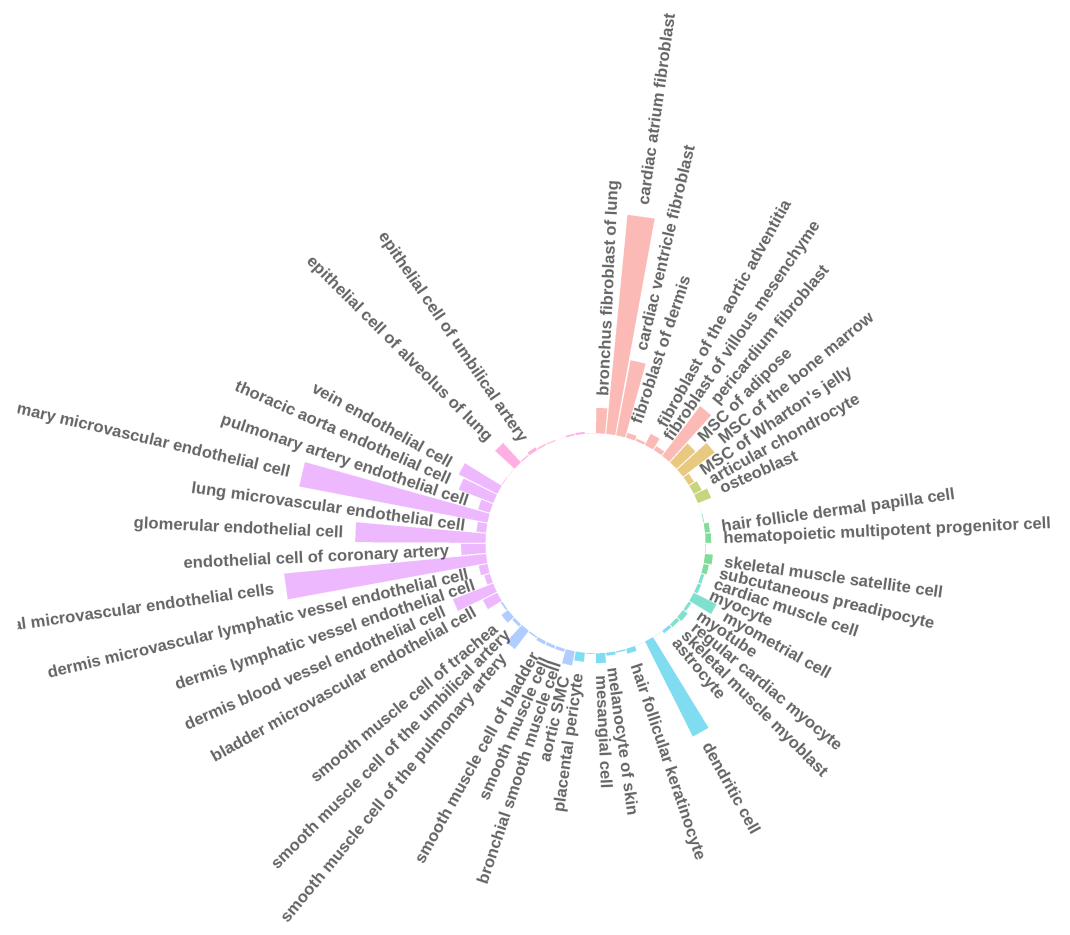

Supplementary Figure 4: Relative expression of 3 positive markers of ENG (CD105) across ENCODE's ribodepleted RNAseq datas, made by K-mer quantification, normalized in kmer by million 


\section{NT5E-ENST00000257770}

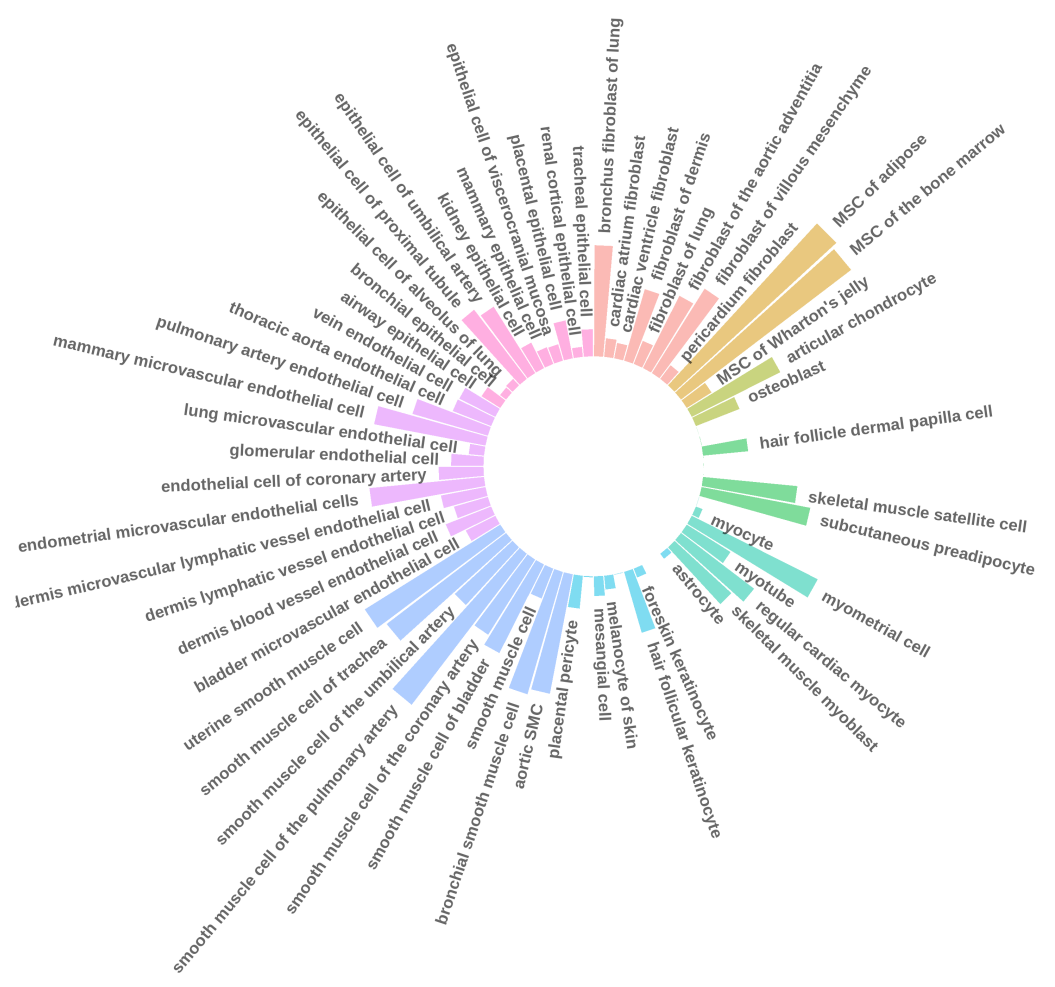

Supplementary Figure 5: Relative expression of 3 positive markers of NT5E (CD73) across ENCODE's ribodepleted RNAseq datas, made by K-mer quantification, normalized in kmer by million 


\section{THY1-ENST00000284240}

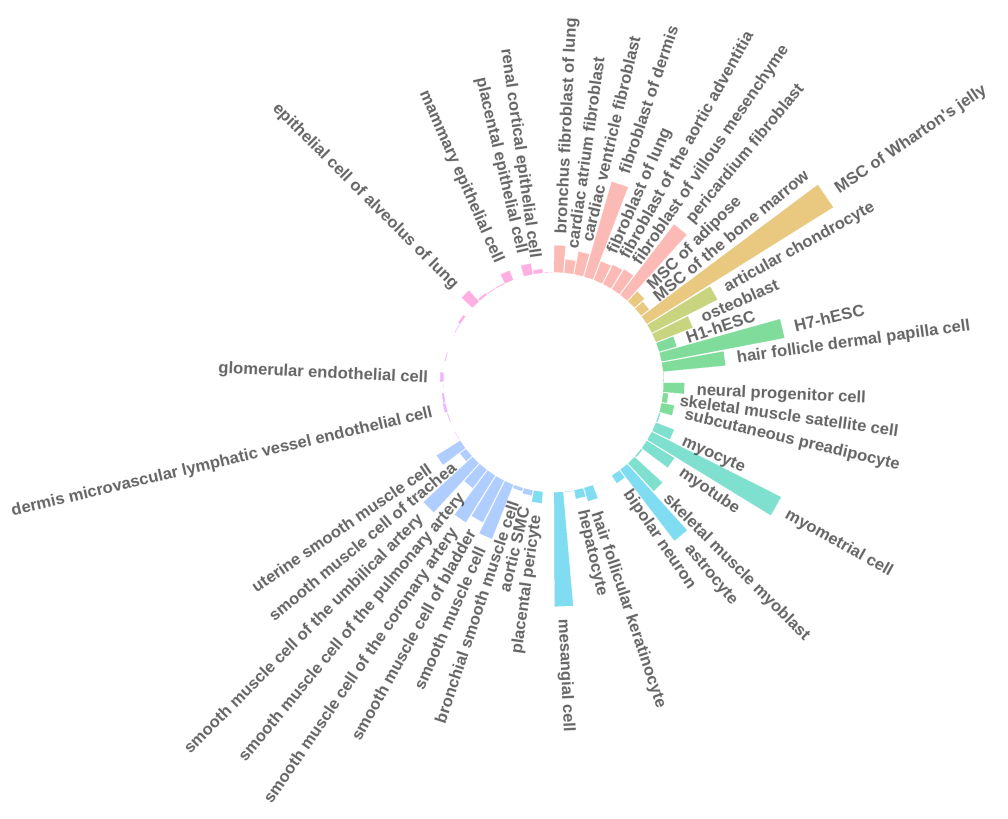

Supplementary Figure 6: Relative expression of 3 positive markers of THY1 (CD90) across ENCODE ribodepleted RNAseq datas, made by K-mer quantification, normalized in kmer by million 


\section{MlincV4.64225.1}

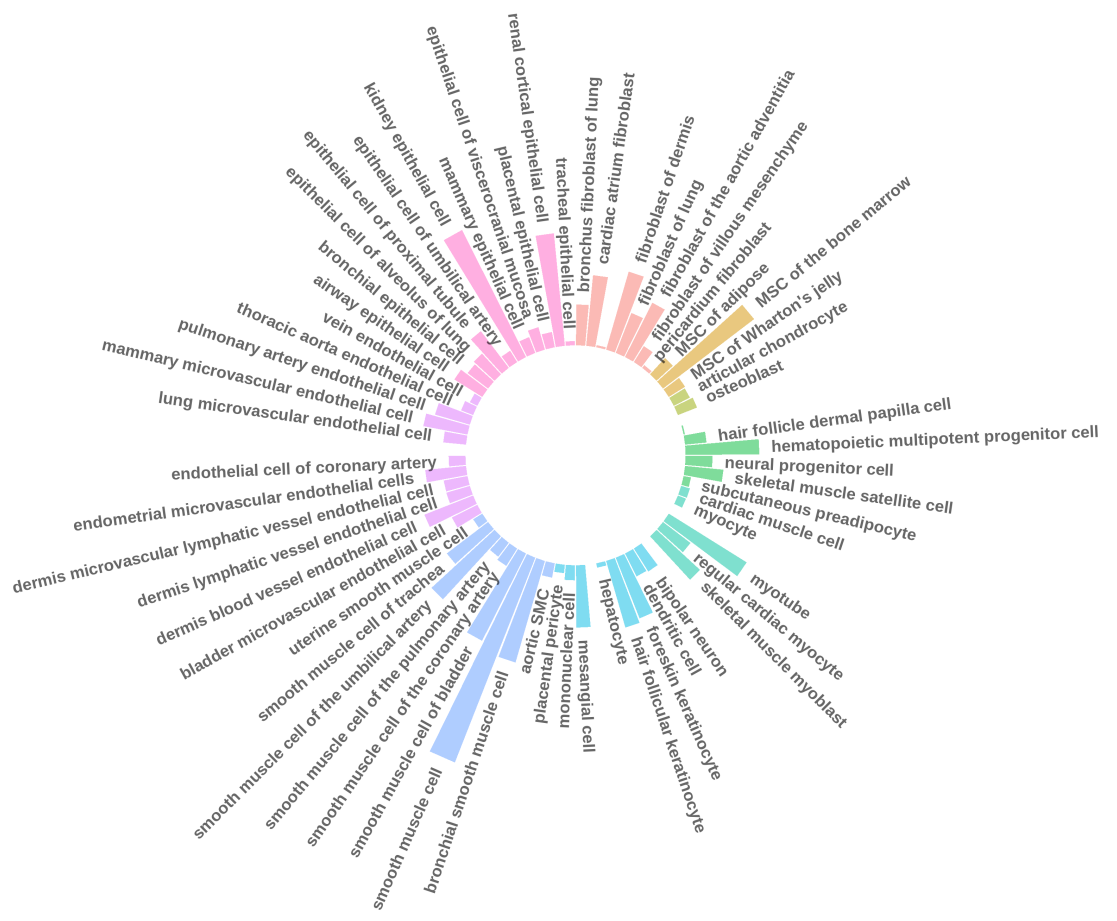

Supplementary Figure 7: Relative expression of 3 positive markers of Mlinc.64225.1 across ENCODE's ribodepleted RNAseq datas, made by Kmer quantification, normalized in kmer by million 


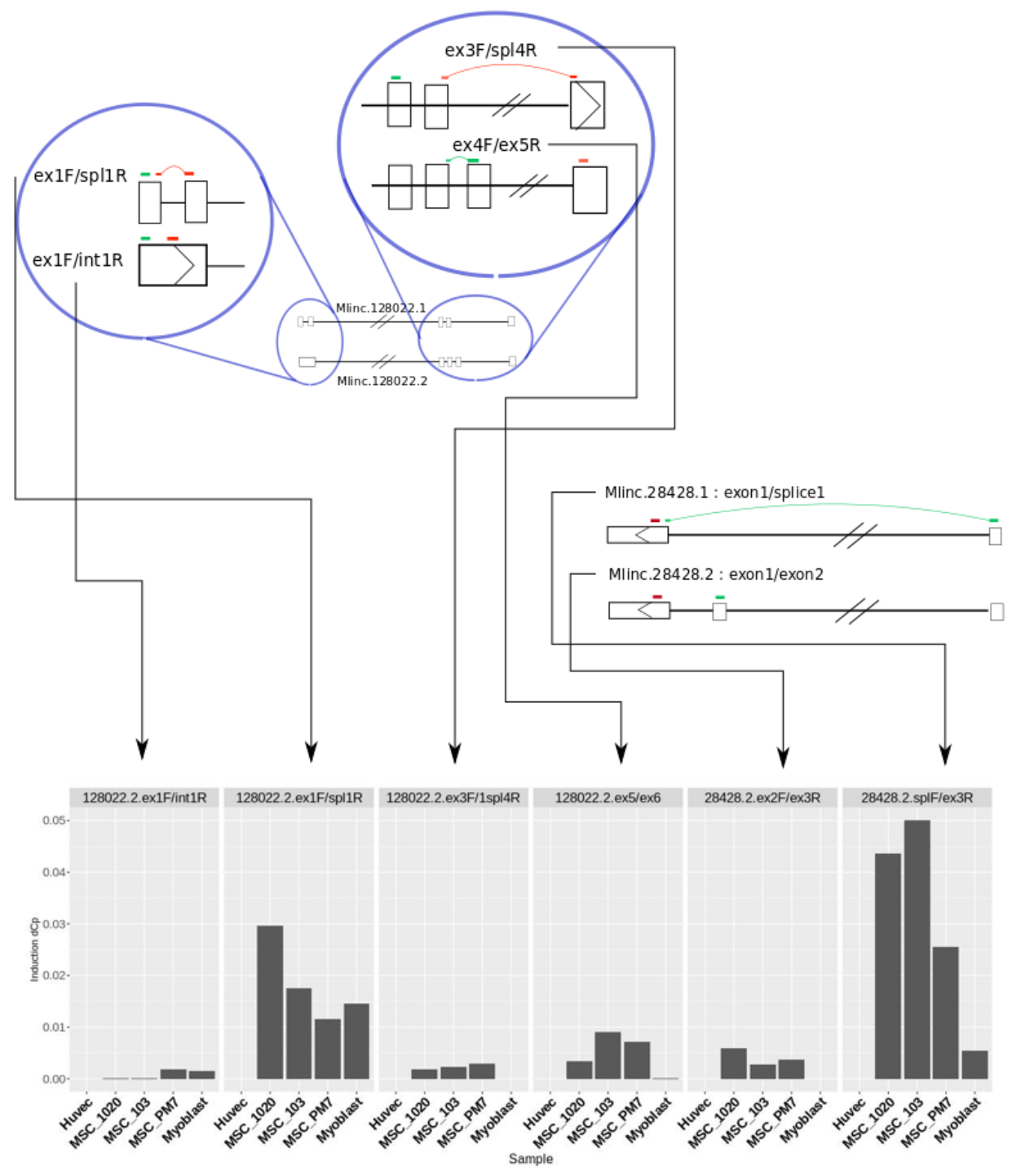

Supplementary Figure 8: Primer position on selected Mlinc candidates and corresponding expression in MSCs, HUVECs and Myoblasts 
bioRxiv preprint doi: https://doi.org/10.1101/2020.03.09.976001; this version posted March 11, 2020. The copyright holder for this preprint (which was not certified by peer review) is the author/funder, who has granted bioRxiv a license to display the preprint in perpetuity. It is made available under aCC-BY-ND 4.0 International license.
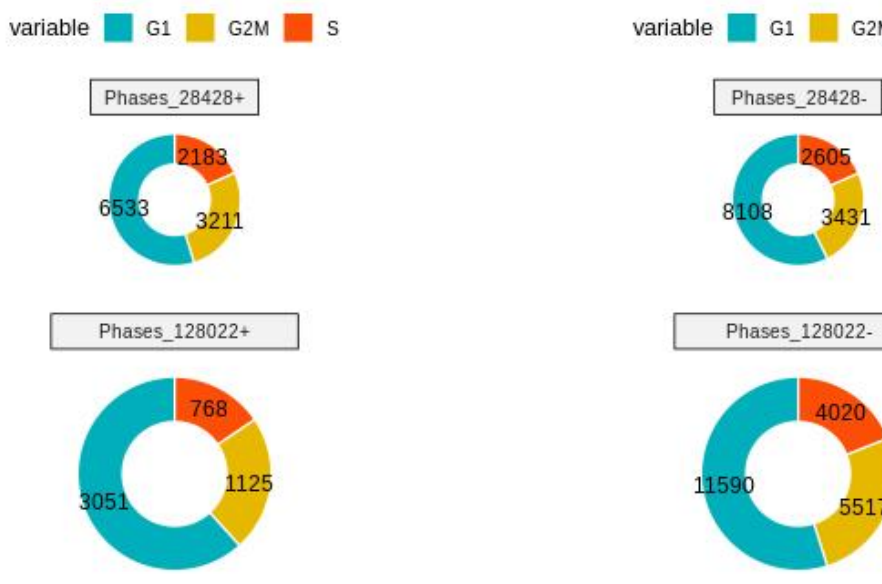

Phases_89912+
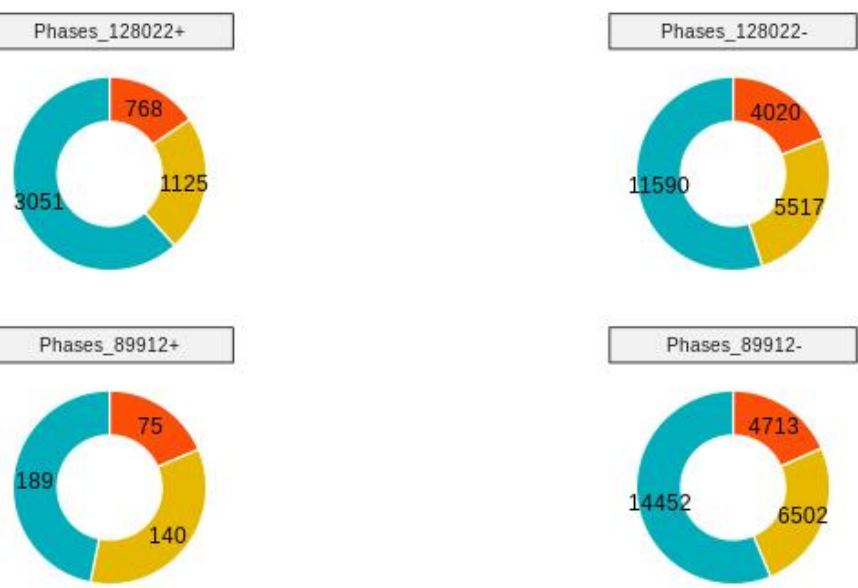

Supplementary Figure 9: cell cycle and single cell. 
Table_S1 Metadata for differential analysis

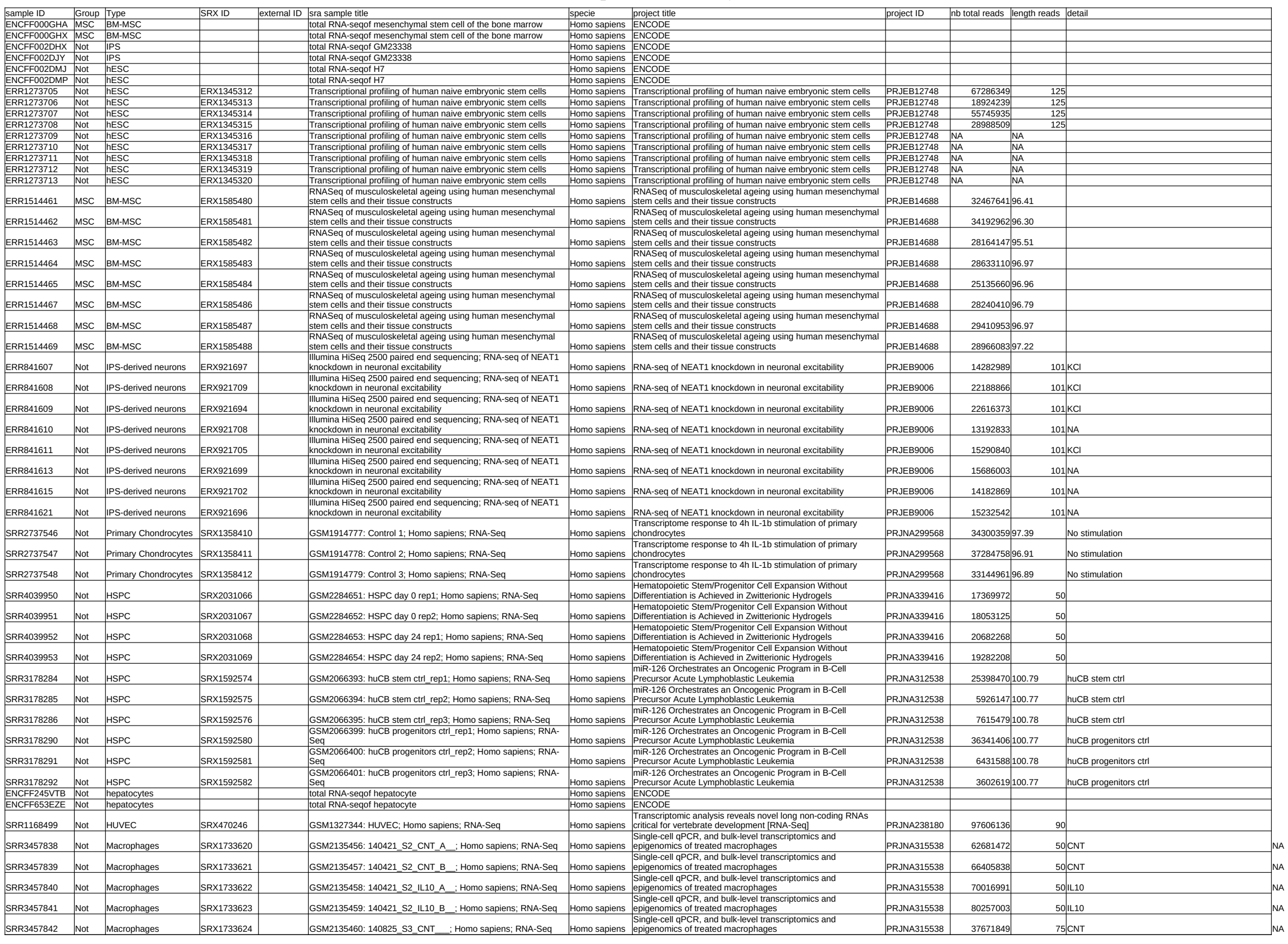


Table_S1 Metadata for differential analysis

\begin{tabular}{|c|c|c|c|c|c|c|c|c|c|}
\hline SRR3457843 & Not & Macrophages & SRX1733625 & GSM2135461: 140825_S3_IL10_; Homo sapiens; RNA-Seq & Homo sapiens & $\begin{array}{l}\begin{array}{l}\text { Single-cell GPCR, and bulk-level transcriptomics and } \\
\text { epigenomics of treated macrophages }\end{array} \\
\end{array}$ & PRJNA315538 & 40575802 & 75|| 110 \\
\hline SRR3457844 & Not & Macrophages & SRX1733626 & GSM2135462: 140825_S1_CNT_; Homo sapiens; RNA-Seq & Homo sapiens & $\begin{array}{l}\text { Single-cell qPCR, and bulk-elevel transscriptomics and } \\
\text { epigignonics of treated macrophages }\end{array}$ & PRJNA315538 & 28548046 & 75 CNT \\
\hline SRR3457845 & Not & Macrophages & SRX1733627 & GSM2135463: 140825_S1_IL10_; Homo sapiens; RNA-Seq & Homo sapiens & $\begin{array}{l}\text { Single-cell qPCR, and bulk-level transcriptomics and } \\
\text { epigenomics of treated macrophages }\end{array}$ & PRJNA315538 & 44848576 & $75 \| 110$ \\
\hline SRR3457846 & Not & Macrophages & SRX1733628 & $\begin{array}{l}\text { GSM2135464: 140825_S1_FFNTFFa_; Homo sapiens; RNA- } \\
\text { Seq }\end{array}$ & Homo sapiens & $\begin{array}{l}\text { lingle-cell qPCR, and bulk-level transcriptomics and } \\
\text { epigenomics of treated macrophages }\end{array}$ & PRJNA315538 & 33920601 & 75 |FNgTNFa \\
\hline SRR3457847 & Not & Macrophages & SRX1733629 & $\begin{array}{l}\text { GSM2135465: 140825_S2_IFNgTNFa_; Homo sapiens; RNA- } \\
\text { Seq }\end{array}$ & Homo sapiens & $\begin{array}{l}\text { ligngle-cell GPCR, and bulk-level transcriptomics and } \\
\text { epigenomics of treated macrophages }\end{array}$ & PRJNA315538 & 26580763 & 75||FNgTNFa \\
\hline SRR3457848 & Not & Macrophages & SRX1733630 & $\begin{array}{l}\text { GSM213 } \\
\text { Seq }\end{array}$ & Homo sapiens & $\begin{array}{l}\text { Single-cell qPCR, and bulk-level transcriptomics and } \\
\text { epigenomics of treated macrophages }\end{array}$ & PRJNA315538 & 28565135 & 75 |FNgTNFa \\
\hline SRR3457849 & Not & Macrophages & SRX1733631 & GSM2135467: 140825_S2_CNT_; Homo sapiens; RNA-Seq & Homo sapiens & $\begin{array}{l}\text { Single-cell qPCR, and bulk-level transcriptomics and } \\
\text { epigenomics of treated macrophages }\end{array}$ & PRJNA315538 & 41595399 & $75 \mathrm{CNT}$ \\
\hline SRR3457850 & Not & Macrophages & SRX1733632 & GSM2135468: 140825_S2_IL10_; Homo sapiens; RNA-Seq & Homo sapiens & $\begin{array}{l}\text { Single-cell qPCR, and bulk-level transcriptomics and } \\
\text { epigenomics of treated macrophages }\end{array}$ & PRJNA315538 & 38443748 & $75 \| 110$ \\
\hline SRR5750514 & Not & hepatocytes & SRX2950738 & Dual RNAseq: Human hepatocytes and Plasmodium berghei & Homo sapiens & $\begin{array}{l}\begin{array}{l}\text { Dual RNAseq - Human hepatocytes infected with Plasmodium } \\
\text { berghei }\end{array} \\
\end{array}$ & PRJNA390648 & \begin{tabular}{l|l|l}
6298345299.3 \\
\end{tabular} & Infected with Plasmodium berghei \\
\hline SRR5750515 & Not & hepatocytes & SRX2950737 & Dual RNAseq: Human hepatocytes and Plasmodium berghei & Homo sapiens & $\begin{array}{l}\text { Dual RNA } \\
\text { berghei }\end{array}$ & PRJNA390648 & \begin{tabular}{|l|l|}
46318597 & 99.3 \\
\end{tabular} & NA \\
\hline SRR5750517 & Not & hepatocytes & SRX2950735 & Dual RNAseq: Human hepatocytes and Plasmodium berghei & Homo sapiens & cytes infected with Plasmodium & PRJNA390648 & 6339146499.2 & NA \\
\hline SRR5750520 & Not & hepatocytes & SRX2950732 & Dual RNAseq: Human hepatocytes and Plasmodium berghei & Homo sapiens & $\begin{array}{l}\text { Dual RNAseq - Human hepatocytes infected with Plasmodium } \\
\text { berghei }\end{array}$ & PRJNA390648 & 5701098899.3 & NA \\
\hline SRR5750521 & Not & hepatocytes & SRX2950731 & Dual RNAseq: Human hepatocytes and Plasmodium berghei & Homo sapiens & 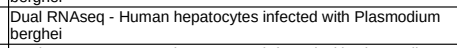 & PRJNA390648 & 4765145699.4 & Infected with Plasmodium berghei \\
\hline SRR5750524 & Not & hepatocytes & SRX2950728 & Dual RNAseq: Human hepatocytes and Plasmodium berghei & Homo sapiens & $\begin{array}{l}\text { Dual RNAseq - Human hepatocytes infected with Plasmodium } \\
\text { berghei }\end{array}$ & PRJNA390648 & 4748667499.5 & NA \\
\hline SRR5750525 & Not & hepatocytes & SRX2950727 & Dual RNAseq: Human hepatocytes and Plasmodium berghei & Homo sapiens & $\begin{array}{l}\text { Dual RNAseq - Human hepatocytes infected with Plasmodium } \\
\text { berghei }\end{array}$ & PRJNA390648 & 5273615299.4 & Infected with Plasmodium berghei \\
\hline SRR5750526 & Not & hepatocytes & SRX2950726 & Dual RNAseq: Human hepatocytes and Plasmodium berghei & Homo sapiens & Human hepatocytes infected with Plasmodium & PRJNA390648 & 4921642099.1 & NA \\
\hline SRR6300667 & Not & HUVEC & SRX3401581 & GSM2859866: hyp_12h_n1_1; Homo sapiens; RNA-Seq & Homo sapiens & 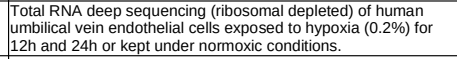 & PRJNA418883 & 39712730 & 94 Hypoxia $0.2 \%$ O2, $5 \%$ CO2 humidified atmosphere \\
\hline SRR6300668 & Not & HUVEC & SRX3401582 & GSM2859867: hyp_12h_n2_1; Homo sapiens; RNA-Seq & Homo sapiens & 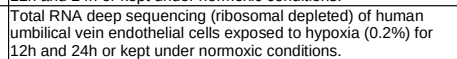 & PRJNA418883 & 43357452 & 94 Hypoxia $0.2 \% \mathrm{O} 2,5 \%$ CO2 humidified atmosphere \\
\hline SRR6300669 & Not & HUVEC & SRX3401583 & GSM2859868: hyp_24h_n1_1; Homo sapiens; RNA-Seq & Homo sapiens & 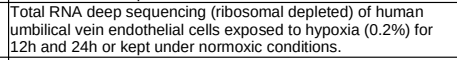 & PRJNA418883 & 41045511 & 94|Hypox \\
\hline SRR6300670 & Not & HUVEC & SRX3401584 & GSM2859869: hyp_24h_n2_1; Homo sapiens; RNA-Seq & Homo sapiens & 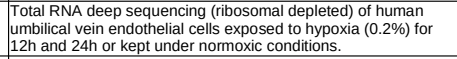 & PRJNA418883 & 44557122 & 94/Hypoxia $0.2 \%$ O2, $5 \%$ CO2 humidified atmosphere \\
\hline SRR6300671 & Not & HUVEC & SRX3401585 & GSM2859870: norm_12h n1 1; Homo sapiens; RNA-Seq & Homo sapiens & 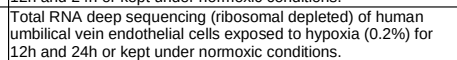 & PRJNA418883 & 42304183 & 94 Normoxia 20\% 02, 5\% CO2 humidified atmosphere \\
\hline SRR6300672 & Not & HUVEC & SRX3401586 & GSM2859871: norm_12h_n2__1; Homo sapiens; RNA-Seq & Homo sapiens & 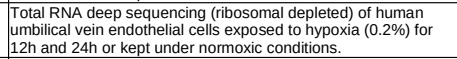 & PRJNA418883 & 427486 & 94|Normoxia \\
\hline SRR6300673 & Not & HUVEC & SRX3401587 & GSM2859872: norm_24h_n1_1; Homo sapiens; RNA-Seq & Homo sapiens & 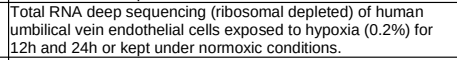 & PRJNA418883 & 42771271 & 94| Normoxia 20\% O2, 5\% CO2 humidified atmosphere \\
\hline SRR6300674 & Not & HUVEC & SRX3401588 & GSM2859873: norm_24h_n2_1; Homo sapiens; RNA-Seq & Homo sapiens & 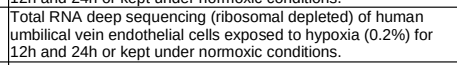 & PRJNA418883 & 42131852 & 944 Normoxia 20\% O2, $5 \%$ CO2 humidified atmosphere \\
\hline SRR3724617 & Not & Lymphocytes & SRX1881712 & GSM2218995: Healthy1-CD4Memory; Homo sapiens; RNA-Seq & Homo sapiens & $\begin{array}{l}\text { Tcells from paroxysmal nocturnal hemoglobinuria patients she } \\
\text { an atatered TNRF signaling pathway }\end{array}$ & PRJNA327044 & 29296358 & 100 healthy \\
\hline SRR3724618 & Not & Lymphocytes & SRX1881713 & GSM2218996: Healthy1-CD4Naive; Homo sapiens; RNA-Seq & Homo sapiens & $\begin{array}{l}\text { Tcells from paroxysmal hocturnal hemoglobinuria patients show } \\
\text { an altered TNFR signaling pathway }\end{array}$ & PRJNA327044 & 27598581 & 100 healthy \\
\hline SRR3724619 & Not & Lymphocytes & SRX1881714 & GSM2218997: Heathy1-CD8Memory; Homo sapiens; RNA-Seq & Homo sapiens & $\begin{array}{l}\text { Tcells from paroxysmal nocturnal hemoglobinuria patients show } \\
\text { an antered TNFR singaling anthway }\end{array}$ & PRJNA327044 & 26351498 & 100 healthy \\
\hline SRR3724620 & Not & Lymphocytes & SRX1881715 & GSM2218998: Healthy1-CDBNaive; Homo sapiens; RNA-Seq & Homo sapiens & $\begin{array}{l}\text { Trells from paraxysmal nocturnal hemoglobinuria patients sh } \\
\text { an altered TNFR signaling pathway }\end{array}$ & PRJNA327044 & 29464342 & 100 healthy \\
\hline SRR3724621 & Not & Lymphocytes & SRX1881716 & GSM2218999: Healthy2-CD4Memory; Homo sapiens; RNA-Seq & Homo sapiens & $\begin{array}{l}\text { Tcells from paroxysmal nocturnal hem } \\
\text { an altered TNFR signaling pathway }\end{array}$ & PRJNA327044 & 48167581 & 100 healthy \\
\hline SRR3724622 & Not & Lymphocytes & SRX1881717 & GSM2219000: Healthy2-CD4Naive; Homo sapiens; RNA-Seq & Homo sapiens & 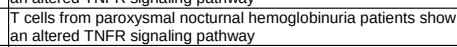 & PRJNA327044 & 42220340 & 100 healthy \\
\hline SRR3724623 & Not & Lymphocytes & SRX1881718 & GSM2219001: Heathy2-CD8Memory; Homo sapiens; RNA-Seq & Homo sapiens & $\begin{array}{l}\text { Trells from paroxysmal nocturnal hemoglobinuria patients show } \\
\text { an altered TNFR signaling pathway }\end{array}$ & PRJNA327044 & 38563382 & 100 healthy \\
\hline SRR3724624 & Not & Lymphocytes & SRX1881719 & GSM2219002: Healthy2-CD8Naive; Homo sapiens; RNA-Seq & Homo sapiens & $\begin{array}{l}\text { Tcells from paroxysmal nocturnal hemoglobinuria patients st } \\
\text { an altered TNFR signaling pathway }\end{array}$ & PRJNA327044 & 45267949 & 100healthy \\
\hline SRR3724625 & Not & Lymphocytes & SRX1881720 & GSM2219003: Heathy3-CD4Memory; Homo sapiens; RNA-Seq & Homo sapiens & $\begin{array}{l}\text { Trells from paroxysmal nocturnal hemo } \\
\text { an altered TNFR signaling pathway }\end{array}$ & PRJNA327044 & 45930063 & 100healthy \\
\hline SRR3724626 & Not & Lymphocytes & SRX1881721 & GSM2219004: Healthy3-CDANaive; Homo sapiens; RNA-Seq & Homo sapiens & $\begin{array}{l}\text { Trells from paroxysmal nocturnal hemoglobinuria patients show } \\
\text { an altered TNFR signaling pathway }\end{array}$ & PRJNA327044 & 40731526 & 100/nealthy \\
\hline SRR3724627 & Not & Lymphocytes & SRX1881722 & GSM2219005: Healthy3-CD8Memory; Homo sapiens; RNA-Seq & Homo sapiens & $\begin{array}{l}\text { Trells from paroxsymal noctural hemoglobinuria patients sho } \\
\text { an altered TNFR signaling pathway }\end{array}$ & PRJNA327044 & 47542258 & 100|healthy \\
\hline SRR3724628 & Not & Lymphocytes & SRX1881723 & GSM2219006: Healthy3-CD8Naive; Homo sapiens; RNA-Seq & Homo sapiens & $\begin{array}{l}\text { Tc cells from paroxysmal nocturnal hemoglobinuria patients show } \\
\text { an altered TNFR signaling pathway }\end{array}$ & PRJNA327044 & 53718245 & 100 healthy \\
\hline SRR3724629 & Not & Lymphocytes & SRX1881724 & GSM2219007: PNH1-CD4Memory; Homo sapiens; RNA-Seq & Homo sapiens & $\begin{array}{l}\text { Tcells from paroxysmal nocturnal hemoglobinuria patients sh } \\
\text { an altered TNFR signaling pathway }\end{array}$ & PRJNA327044 & 30025375 & 100|paroxysmal nocturnal hemog \\
\hline SRR3724630 & Not & Lymphocytes & SRX1881725 & GSM2219008: PNH1-CD4Naive; Homo sapiens; RNA-Seq & Homo sapiens & $\begin{array}{l}\text { Tcells from paroxysmal nocturnal hem } \\
\text { an altered TNFR signaling pathway }\end{array}$ & PRJNA327044 & 29638535 & 100 paroxysmal nocturnal hemoglobinuria $(\mathrm{PNH})$ \\
\hline SRR3724631 & Not & Lymphocytes & SRX1881726 & GSM2219009: PNH1-CD8Memory; Homo sapiens; RNA-Seq & Homo sapiens & $\begin{array}{l}\text { Tr cells from paroxysmal nocturnal hem } \\
\text { an altered TNFR signaling pathway }\end{array}$ & PRJNA327044 & 30553368 & ${ }_{100}$ paroxysmal nocturnal hemoglobinuria (PNH) \\
\hline SRR3724632 & Not & Lymphocytes & SRX1881727 & GSM2219010: PNH1-CD8Naive; Homo sapiens; RNA-Seq & Homo sapiens & $\begin{array}{l}\text { Te cells from paroxysmal nocturnal hemoglobinuria pe } \\
\text { an antered TNRR signaling pathway }\end{array}$ & PRJNA327044 & 34722293 & 100 paroxysmal nocturnal hemoglobinuria (PNH) \\
\hline
\end{tabular}


Table_S1 Metadata for differential analysis

\begin{tabular}{|c|c|c|c|c|c|c|c|c|c|}
\hline RR3724633 & Not & Lymphocytes & SRX1881728 & GSM2219011: PNH2-CD4Memory; Homo sapiens; RNA-Seq & & \begin{tabular}{|l} 
Tcells from paroxysmal nocturnal hemoglobinuria patients show \\
an altered TNFR signaling pathway
\end{tabular} & 4327044 & 32466930 & 100 paroxysmal nocturnal hemoglobinuria (PNH) \\
\hline SRR3724634 & Not & -ymphocytes & SRX1881729 & GSM2219012: PNH2-CD4Naive; Homo sapiens; RNA-Seq & Homo sapiens & $\begin{array}{l}\text { Tcells from paroxysmal nocturnal hemoglobinuria patients show } \\
\text { an altered TNFR signaling pathway }\end{array}$ & PRJNA327044 & 29139809 & 100 paroxysmal nocturnal hemoglobinuria (PNH) \\
\hline SRR3724635 & Not & Lymphocytes & SRX1881730 & GSM2219013: PNH2-CD8Memory; Homo sapiens; RNA-Seq & Homo sapiens & $\begin{array}{l}\text { Trells from paraxysmal nocturnal he } \\
\text { an altered TNFR signaling pathway } \\
\end{array}$ & PRJNA327044 & 28506728 & 100 paroxysmal nocturnal hemoglobinuria (PNH) \\
\hline SRR3724636 & Not & Lymphocytes & SRX1881731 & GSM2219014: PNH2-CD8Naive; Homo sapiens; RNA-Seq & Homo sapiens & $\begin{array}{l}\text { Tc cells from maroxxymal nocturnal her } \\
\text { an altered TNFR signaling pathway }\end{array}$ & PRJNA327044 & 30666327 & 100 paroxysmal nocturnal hemoglobinuria (PNH) \\
\hline SRR3724637 & Not & Lymphocytes & SRX1881732 & GSM2219015: PNH3-CD4Memory; Homo sapiens; RNA-Seq & Homo sapiens & $\begin{array}{l}\text { Tcells from paroxysmal noctural hemog } \\
\text { an altered TNRR signaling pathway }\end{array}$ & PRJNA327044 & 49485440 & 100 paroxysmal nocturnal hemog \\
\hline SRR3724638 & Not & Lymphocytes & SRX1881733 & GSM2219016: PNH3-CD4Naive; Homo sapiens; RNA-Seq & Homo sapiens & $\begin{array}{l}\text { Taroxysmal nocturnal hemoglobinu } \\
\text { NNFR signaling pathway }\end{array}$ & PRJNA327044 & 43428307 & 100 paroxysmal nocturnal hemoglobinuria (PNH) \\
\hline RR3724639 & Not & Lymphocytes & SRX1881734 & GSM2219017: PNH3-CD8Memory; Homo sapiens; RNA-Seq & Homo sapiens & $\begin{array}{l}\text { Tcells from paroxysmal nocturnal he } \\
\text { an altered TNFR signaling pathway }\end{array}$ & PRJNA327044 & 41998683 & 100 paroxysmal nocturnal hemogl \\
\hline SRP3724640 & Not & vompoytes & $\mathrm{SPX} 1881735$ & GSM2219018. PNH3-CD8Naive. Homo saniens. RNA-Seg & Homo saniens & 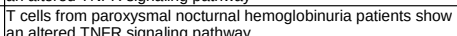 & PRJNA327044 & 52622177 & (Dol \\
\hline
\end{tabular}


Table_S2 Metadata for ENCODE kmer research

\begin{tabular}{|c|c|c|c|c|c|c|c|c|c|c|c|c|}
\hline Original.files & label & group & Accession & Assay.Nickname & Biosample.summary & Lab & Project & Species & Life.stage & Age & Age.Units & Treatment \\
\hline ENCFF371KWE & dendritic cell & 6: Others & ENCSR892ZUK & total RNA-seq & $\begin{array}{l}\text { dendratitic celt treated with } 100 \mathrm{ng} / \mathrm{mL} \\
\text { Lipopolysaccharide for } 2 \text { hours }\end{array}$ & Manuel Garber, UMass & GGR & Homo sapiens & unknown & unknown & & Lipopolysaccharide \\
\hline ENCFF534HFO & dendritic cell & 6: Others & ENCSR519NFO & total RNA-seq & $\begin{array}{l}\text { dendriticic ecll treated with } 1000 \text { gn/mL } \\
\text { Lipopolysaccharide for } 1 \text { hour }\end{array}$ & Manuel Garber, UMass & GGR & Homo sapiens & unknown & unknown & & Lipopolysaccharide \\
\hline ENCFF126HPJ & $\begin{array}{l}\text { endometrial microvascular } \\
\text { endothelial cells }\end{array}$ & 8: Endothelial cells & ENCSR919MZM & total RNA-seq & $\begin{array}{l}\text { endometrial microvascular endott } \\
\text { female adult (34 years) }\end{array}$ & Thomas Gingeras, CSHL & ENCODE & Homo sapiens & adult & & year & \\
\hline ENCFF502JCS & $\begin{array}{l}\text { endometrial microvascular } \\
\text { endothelial cells }\end{array}$ & 8: Endothelial cells & ENCSR919MZM & total RNA-seq & $\begin{array}{l}\text { endometrial microvascular endothelial cells } \\
\text { female adult } 33 \text { years }\end{array}$ & Thomas Gingeras, CSHL & ENCODE & Homo sapiens & adult & & year & \\
\hline ENCFF001QZS & $\begin{array}{l}\text { Smooth muscle cell of } \\
\text { bladder }\end{array}$ & 7: SMC & ENCSROOOAAC & total RNA-seq & $\begin{array}{l}\text { smooth muscle cell of bladder female ad } \\
\text { (533 years) and male adult (62 years) }\end{array}$ & Thomas Gingeras, CSHL & ENCODE & Homo sapiens & adult & 53,62 & year & \\
\hline ENCFF001RAO & $\begin{array}{l}\text { Smotot muscle cell of } \\
\text { bladder }\end{array}$ & 7: SMC & ENCSROOOAAC & total RNA-seq & $\begin{array}{l}\text { smooth muscle cell of bladder female adult } \\
\text { (53 years) and male adult (62 years) }\end{array}$ & Thomas Gingeras, CSHL & ENCODE & Homo sapiens & adult & 53,62 & year & \\
\hline ENCFF001QZC & renal cortical epithelial cell & 9: Epithelial cells & ENCSRO0OAAQ & total RNA-seq & $\begin{array}{l}\text { renana cortical eithelialal cell female adult (69 } \\
\text { years) and male adutt (84 years) }\end{array}$ & Thomas Gingeras, CSHL & ENCODE & Homo sapiens & adult & 69,84 & year & \\
\hline ENCFF001RAU & renal cortical epithelial cell & 9: Epithelial cells & ENCSROOOAAQ & total RNA-seq & 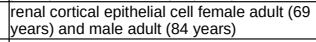 & Thomas Gingeras, CSHL & ENCODE & Homo sapiens & adult & 69,84 & Iyear & \\
\hline ENCFF001QZK & $\begin{array}{l}\text { bladder riarcovascular } \\
\text { endothelial cell }\end{array}$ & 8: Endothelial cells & ENCSROOOAAB & total RNA-seq & $\begin{array}{l}\text { bladder microvascular endothelial cell male } \\
\text { adult ( } 46 \text { years) and male addit ( } 60 \text { years) }\end{array}$ & Thomas Gingeras, CSHL & ENCODE & Homo sapiens & adult & 46,6 & year & \\
\hline ENCFF001RAI & $\begin{array}{l}\text { bladder microvascular } \\
\text { endothelial cell }\end{array}$ & 8: Endothelial cells & ENCSRO0OAAB & total RNA-seq & $\begin{array}{l}\text { bladder microvascular endothelial cell male } \\
\text { adult } 446 \text { years) and male adult ( }(60 \text { years) }\end{array}$ & Thomas Gingeras, CSHL & ENCODE & Homo sapiens & adult & 46 & year & \\
\hline ENCFF001QZG & $\begin{array}{l}\text { dermis blood vessel } \\
\text { endothelial cell }\end{array}$ & 8: Endothelial cells & ENCSRO0OAAI & total RNA-seq & $\begin{array}{l}\text { dermis blood vessel endothelial cell female } \\
\text { child (16 years) and male child (13 years) }\end{array}$ & Thomas Gingeras, CSHL & ENCODE & Homo sapiens & child & 13,1 & year & \\
\hline ENCFF001RAQ & $\begin{array}{l}\text { dermis blood vessel } \\
\text { endothelial cell }\end{array}$ & 8: Endothelial cells & ENCSRO00AAI & total RNA-seq & $\begin{array}{l}\text { dermis blood vessel endothelial cell female } \\
\text { child (11 years) and male child }(13 \text { years) }\end{array}$ & Thomas Gingeras, CSHL & ENCODE & Homo sapiens & child & 13., & year & \\
\hline ENCFF00OILE & skeletal muscle satellite ce & 4: Progenitor or Stem cell & ENCSROOOCUI & total RNA-seq & $\begin{array}{l}\text { lakeltal muscle satellite cell fema } \\
\text { years) and male adult (21 year) }\end{array}$ & Thomas Gingeras, CSHL & ENCODE & Homo sapiens & adult & 21,64 & year & \\
\hline ENCFFOOOILQ & skeletal muscle satellite ce & 14: Progenitor or $S$ & ENCSROOOCUI & total RNA-seq & $\begin{array}{l}\text { skeletal muscle satellite cell female } \\
\text { years and male edult (21 year) }\end{array}$ & Thomas Gingeras, CSHL & ENCODE & Homo sapiens & adult & & year & \\
\hline ENCFFO0OGFS & mononuclear cell & 6: Others & ENCSROOOCUT & total RNA-seq & 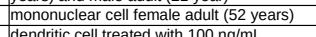 & Thomas Gingeras, CSHL & ENCODE & Homo sapiens & adult & & year & \\
\hline ENCFF810KXA & dendritic cell & 6: Others & ENCSR682CFV & total RNA-seq & 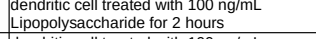 & Manuel Garber, UMass & GGR & Homo sapiens & unknown & unknown & & Lipopolysaccharide \\
\hline ENCFF928LQX & dendritic cell & 6: Others & ENCSR652RSO & total RNA-seq & $\begin{array}{l}\text { dendiritic cell treated with } 100 \mathrm{ng} / \mathrm{mL} \\
\text { Lipopolysaccharide for } 6 \text { hours }\end{array}$ & Manuel Garber, UMass & GGR & Homo sapiens & unknown & unknown & & Lipopolysaccharide \\
\hline ENCFF816SSU & dendritic cell & 6: Others & ENCSR536GUD & total RNA-seq & $\begin{array}{l}\text { dendriticic cell treated with } 100 \mathrm{ng} / \mathrm{mL} \\
\text { Lipopolysaccharide for } 4 \text { hours }\end{array}$ & Manuel Garber, UMass & GGR & Homo sapiens & unknown & unknown & & Lipopolysaccharide \\
\hline ENCFF720QTK & dendritic cell & 6: Others & ENCSR870DRE & total RNA-seq & $\begin{array}{l}\text { dendritic cell treated with } 0 \mathrm{ng} / \mathrm{mL} \\
\text { Lipopolysaccharide for } 0 \text { hours }\end{array}$ & Manuel Garber, UMass & GGR & Homo sapiens & unknown & unknown & & Lipopolysaccharide \\
\hline $\begin{array}{l}\text { ENCFF816QOY } \\
\text { ENFFF46PPC }\end{array}$ & $\begin{array}{ll}\text { H1-hESC } \\
\text { H1-hESC }\end{array}$ & $\begin{array}{l}\text { 4: Progenitor or Stem cell } \\
\text { 4: Progenintor or stem cell }\end{array}$ & 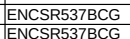 & $\begin{array}{l}\text { total RNA-seq } \\
\text { total }\end{array}$ & $\begin{array}{ll}\text { H1-hESC } \\
\text { H1-hESC }\end{array}$ & $\begin{array}{l}\text { Joe Ecker, Salk } \\
\text { J Je EFker, Salk }\end{array}$ & $\begin{array}{l}\text { Roadmap } \\
\text { Roartman }\end{array}$ & $\begin{array}{l}\text { Homo sapiens } \\
\text { Homo sapiens }\end{array}$ & $\begin{array}{l}\text { embryonic } \\
\text { embronic }\end{array}$ & $\begin{array}{l}\text { unknown } \\
\text { unnknown }\end{array}$ & & \\
\hline $\begin{array}{l}\text { ENCF-468PHC } \\
\text { ENCFF821YWS }\end{array}$ & $\begin{array}{lll}\text { Hil-nESC } \\
\text { H1-hESC }\end{array}$ & $\begin{array}{l}\text { 4. Progentitor or Stem cell } \\
\text { 4: Progenitor or Stem cell }\end{array}$ & $\begin{array}{l}\text { ENSSR53/BCG } \\
\text { ENCSR537BCG }\end{array}$ & total RNA-seq & $\begin{array}{l}\text { H1-hESC } \\
\text { H1-hESC }\end{array}$ & \begin{tabular}{|l} 
Joe ctker, Salk \\
Joe Ecker, Salk
\end{tabular} & $\begin{array}{l}\text { Roaamap } \\
\text { Roadmap }\end{array}$ & $\begin{array}{l}\text { Homm sapens } \\
\text { Homo sapiens } \\
\end{array}$ & $\begin{array}{l}\text { embryonic } \\
\text { embryonic }\end{array}$ & Junknown & & \\
\hline ENCFF712SHP & H1-hESC & 4: Progenitor or Stem cell & ENCSR537BCG & total RNA-seq & $\begin{array}{l}\text { H1-nESC } \\
\text { H1-hESC }\end{array}$ & $\begin{array}{l}\text { Joe Eckel, Salk } \\
\text { Joe Ecker, Salk }\end{array}$ & $\begin{array}{l}\text { Roaamap } \\
\text { Roadmap }\end{array}$ & $\begin{array}{l}\text { Homm sapipens } \\
\text { Homo sapiens }\end{array}$ & $\begin{array}{l}\text { embryonic } \\
\text { embryonic } \\
\end{array}$ & Junknown & & \\
\hline ENCFF793PLC & H1-hESC & 4: Progenitor or Stem cell & ENCSR537BCG & total RNA-seq & H1-hESC & Joe Ecker, Salk & Roadmap & Homo sapiens & embryonic & unknown & & \\
\hline ENCFF000FKR & $\begin{array}{l}\text { thoracic aorta endothelial } \\
\text { cell }\end{array}$ & 8: Endothelial cells & ENCSROOOCUK & total RNA-seq & $\begin{array}{l}\text { thoracicic aorta endothelial cell female a adult } \\
\text { (122 years) and male adult (55 years) }\end{array}$ & Thomas Gingeras, CSHL & ENCODE & Homo sapiens & adult & 55,22 & year & \\
\hline ENCFF000FLI & $\begin{array}{l}\text { thoracic aorta endothelial } \\
\text { cell }\end{array}$ & 8: Endothelial cells & ENCSROOOCUK & total RNA-seq & $\begin{array}{l}\text { thoracicic aorta endothelial cell female adult } \\
(22 \text { years) and male adult (55 years) }\end{array}$ & Thomas Gingeras, CSHL & ENCODE & Homo sapiens & adult & 55,22 & year & \\
\hline ENCFF00OGBO & $\begin{array}{l}\text { hair follicle dermal papilla } \\
\text { cell }\end{array}$ & 4: Progenitor or Stem cell & ENCSRO0OCUB & total RNA-seq & $\begin{array}{l}\text { hair follicle dermal papilla cell female adult } \\
\text { (47 years and femmala adult (70 years) }\end{array}$ & Thomas Gingeras, CSHL & ENCODE & Homo sapiens & adult & 70,47 & year & \\
\hline ENCFF000GBT & $\begin{array}{l}\text { hair follicle dermal papilla } \\
\text { cell }\end{array}$ & 4: Progenitor or Stem cell & ENCSROOOCUB & total RNA-seq & $\begin{array}{l}\text { hair follicle dermal papilla cell female adult } \\
\text { (47 years and anmala adult t77 years }\end{array}$ & Thomas Gingeras. CSHL & ENCODE & Homo sapiens & adult & & year & \\
\hline ENCFF656QHH & & 5: Muscle cells & ENCSR371VGV & total RNA-Seq & myometrial cell female adult (34 years) & Thomas Gingeras, CSHL & ENCODE & $\begin{array}{l}\text { Homo sappens } \\
\text { Homs sains }\end{array}$ & adult & & & \\
\hline ENCFF212TDB & myometrial cell & 5: Muscle cells & ENCSR371VGV & total RNA-seq & myometrial cell female adult (34 years) & Thomas Gingeras, CSHL & ENCODE & Homo sapiens & adult & & year & \\
\hline ENCFF995TMT & dendritic cell & 6: Others & ENCSR332SRS & total RNA-seq & $\begin{array}{l}\text { dendiritic ell treated with } 100 \mathrm{ng} / \mathrm{mL} \\
\text { Lipopolysachcharide for } 4 \text { hoours }\end{array}$ & Manuel Garber, UMass & GGR & Homo sapiens & unknown & unknown & & -ipopolysaccharide \\
\hline ENCFF605VBT & glomerular endothelial cell & 8: Endothelial cells & ENCSR878EUT & total RNA-seq & $\begin{array}{l}\text { glomerular endothelial cell female embryo } \\
(22 \text { weeks) and male embryo (22 weeks) }\end{array}$ & Thomas Gingeras, CSHL & ENCODE & Homo sapiens & embryonic & & week & \\
\hline ENCFF509JLH & glomerular endothelial cell & 8: Endothelial cells & ENCSR878EUT & total RNA-seq & $\begin{array}{l}\text { glomerular endotothelial cell temale embryo } \\
\text { (22 weeks) and male embryo (22 weeks) }\end{array}$ & Thomas Gingeras, CSHL & ENCODE & Homo sapiens & embryonic & & week & \\
\hline ENCFF000FLO & articular chondrocyte & character(0) & ENCSROOOCUE & total RNA-seq & $\begin{array}{l}\text { articulala chondrocycte of knee joint female } \\
\text { adult }(56 \text { years) and male adult } 64 \text { years) }\end{array}$ & Thomas Gingeras, CSHL & ENCODE & Homo sapiens & adult & & year & \\
\hline ENCFF000FMM & articular chondrocyte & character(0) & ENCSROOOCUE & total RNA-seq & 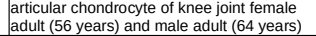 & Thomas Gingeras, CSHL & ENCODE & Homo sapiens & adult & 64,56 & year & \\
\hline ENCFF875NIU & dendritic cell & 6: Others & ENCSR178SNP & total RNA-seq & $\begin{array}{l}\text { dendriticic cell treated with } 100 \mathrm{ng} / \mathrm{mL} \\
\text { Litopolysaccharide for } 1 \text { hoour }\end{array}$ & Manuel Garber, UMass & GGR & Homo sapiens & unknown & unknown & & Lipopolysaccharide \\
\hline ENCFF000GXC & $\begin{array}{l}\text { fibroblast of villous } \\
\text { mesenchyme }\end{array}$ & 1: Fibroblast & ENCSRO0OCUL & total RNA-seq & $\begin{array}{l}\text { fibroblast of villous mesenchyme female } \\
\text { newborn and male newborn }\end{array}$ & Thomas Gingeras, CSHL & ENCODE & Homo sapiens & newborn & unknown & & \\
\hline ENCFF000GXV & $\begin{array}{l}\text { fifroblast of villous } \\
\text { mesenchyme }\end{array}$ & 1: Fibroblast & ENCSROOOCUL & total RNA-seq & $\begin{array}{l}\text { fibroblast of villous mesenchyme female } \\
\text { newborn and male newborn }\end{array}$ & Thomas Gingeras, CSHL & ENCODE & Homo sapiens & newborn & unknown & & \\
\hline ENCFF236EYN & hair follicular keratinocyte & 6: Others & ENCSR680USE & total RNA-seq & $\begin{array}{l}\text { hair follicular keratinocyte male adult (55 } \\
\text { years) }\end{array}$ & Thomas Gingeras, CSHL & ENCODE & Homo sapiens & adult & & Iyear & \\
\hline ENCFF917MHA & hair follicular keratinocyte & 6: Others & ENCSR680USE & total RNA-seq & $\begin{array}{l}{\left[\begin{array}{l}\text { hiai f fllicular keratinocyte male adult (55 } \\
\text { years }\end{array}\right.} \\
\end{array}$ & Thomas Gingeras, CSHL & ENCODE & Homo sapiens & adult & & year & \\
\hline ENCFF142YQX & mesangial cell & 6: Others & ENCSR198TKA & total RNA-seq & $\begin{array}{l}\begin{array}{l}\text { mesangial cell NONE and female embryo } \\
(21 \text { week })\end{array} \\
\end{array}$ & Thomas Gingeras, CSHL & ENCODE & Homo sapiens & unknown,embry & yunknown, 21 & week & \\
\hline ENCFF644UKX & mesangial cell & 6: Others & ENCSR198TKA & total RNA-seq & $\begin{array}{l}\begin{array}{l}\text { mesangial cell NONE and female embryo } \\
(21 \text { week })\end{array} \\
\end{array}$ & Thomas Gingeras, CSHL & ENCODE & Homo sapiens & unknown,embry & yunknown, 21 & week & \\
\hline ENCFF927MXX & myotube & 5: Muscle cells & ENCSR828TEI & total RNA-seq & f from skeletal muscle & Thomas Gingeras, CSHL & ENCODE & Homo sapiens & unknown & unknown & & \\
\hline ENCFF999QZD & myotube & 5: Muscle cells & ENCSR828TEI & total RNA-seq & $\begin{array}{l}\text { myotube originated from skeletal muscle } \\
\text { myoblast }\end{array}$ & Thomas Gingeras, CSHL & ENCODE & Homo sapiens & unknown & unknown & & \\
\hline ENCFF911ELB & foreskin keratinocyte & 6: Others & ENCSR034RPU & total RNA-seq & $\begin{array}{l}\text { foreskin keratinocyte male newborn (2-4 } \\
\text { days) }\end{array}$ & Michael Snyder, Stanford & GGR & Homo sapiens & newborn & $2-4$ & day & \\
\hline ENCFF578CTE & foreskin keratinocyte & 6: Others & ENCSR034RPU & total RNA-seq & $\begin{array}{l}\text { foreskin keratinocyte male newborn (2-4 } \\
\text { days) }\end{array}$ & Michael Snyder, Stanford & GGR & Homo sapiens & newborn & $2-4$ & day & \\
\hline ENCFFOOOGNB & Wvein endothelial cell & 8: Endothelial cells & ENCSRO0OCUG & total RNA-seq & $\begin{array}{l}\text { vein endotheneial cell male adult (48 years } \\
\text { and male a audt (55years) }\end{array}$ & Thomas Gingeras, CSHL & ENCODE & Homo sapiens & adult & 52. & year & \\
\hline
\end{tabular}


Table_S2 Metadata for ENCODE kmer research

\begin{tabular}{|c|c|c|c|c|c|c|c|c|c|c|c|}
\hline ENCFFOOOGNC & vein endothelial cell & 8: Endothelial cells & ENCSROOOCUG & total RNA-seq & $\begin{array}{l}\text { vein endothelial cell male adult (48 years) } \\
\text { and male adult (52 years) }\end{array}$ & Thomas Gingeras, CSHL & ENCODE & Homo sapiens & adult & 52,48 year & \\
\hline ENCFF001QZM & $\begin{array}{l}\text { smooth muscle cell of } \\
\text { rrachea }\end{array}$ & 7: SMC & ENCSRO0OAAS & total RNA-seq & $\begin{array}{l}\text { smooth muscle cell of trachea male adult } \\
\text { (28 years) and male adult (56 years) }\end{array}$ & Thomas Gingeras, CSHL & ENCODE & Homo sapiens & adult & 56,28 year & \\
\hline ENCFF001RDQ & $\begin{array}{l}\text { Smooth muscle cell of } \\
\text { rrachea }\end{array}$ & 7: SMC & ENCSROOOAAS & total RNA-seq & $\begin{array}{l}\text { Smooth muscle cell of trachea male adult } \\
\text { (28 years) and male adult (56 years) }\end{array}$ & Thomas Gingeras, CSHL & ENCODE & Homo sapiens & adult & 56,28 year & \\
\hline ENCFF001RCA & $\begin{array}{l}\text { smooth muscle cell of the } \\
\text { pulmonary artery }\end{array}$ & 7: SMC & ENCSROOOAAN & total RNA-seq & $\begin{array}{l}\text { smooth muscle cell of the pulmonary artery } \\
\text { male adult (26 years) and male adult (28 } \\
\text { years) }\end{array}$ & Thomas Gingeras, CSHL & ENCODE & Homo sapiens & adult & 28,26year & \\
\hline ENCFF001RDO & $\begin{array}{l}\text { smooth muscle cell of the } \\
\text { pulmonary artery }\end{array}$ & 7: SMC & ENCSROOOAAN & total RNA-seq & $\begin{array}{l}\text { 吕ooth muscle cell of the pulmonary artery } \\
\text { male adult (26 years) and male adult ( } 28 \\
\text { years) }\end{array}$ & Thomas Gingeras, CSHL & ENCODE & Homo sapiens & adult & 28,26year & \\
\hline ENCFFO0OGKX & placental pericyte & 6: others & ENCSROoOCTX & total RNA-seq & $\begin{array}{l}\text { placental pericyte female newborn and male } \\
\text { newborn }\end{array}$ & Thomas Gingeras, CSHL & ENCODE & Homo sapiens & newborn & unknown & \\
\hline ENCFF000GKZ & placental pericyte & 6: Others & ENCSRO0OCTX & total RNA-seq & $\begin{array}{l}\text { placental pericyte female newborn and male } \\
\text { newborn }\end{array}$ & Thomas Gingeras, CSHL & ENCODE & Homo sapiens & newborn & unknown & \\
\hline ENCFFOOOHWI & fibroblast of dermis & 1: Fibroblast & ENCSROOOCUH & total RNA-seq & $\begin{array}{l}\text { fibroblast of dermis female adult ( } 44 \text { years) } \\
\text { and female adult (55 years) }\end{array}$ & Thomas Gingeras, CSHL & ENCODE & Homo sapiens & adult & 55,44 year & \\
\hline ENCFFOOOHXB & fibroblast of dermis & 1: Fibroblast & ENCSROOOCUH & total RNA-seq & $\begin{array}{l}\text { fibroblast of dermis female adult ( } 44 \text { years) } \\
\text { and female adult (55 years) }\end{array}$ & Thomas Gingeras, CSHL & ENCODE & Homo sapiens & adult & 55,44 year & \\
\hline ENCFF644CJJ & bipolar neuron & 6: Others & ENCSR968WKR & total RNA-seq & $\begin{array}{l}\text { bipolar neuron originated from GM233338 } \\
\text { treated with } 0.5 \mathrm{gg} / \mathrm{mL} \text { doxycycline hyclate } \\
\text { for } 4 \text { days }\end{array}$ & Thomas Gingeras, CSHL & ENCODE & Homo sapiens & adult & eear & doxycycline hyclate \\
\hline \begin{tabular}{|l|} 
ENCFF040TFC \\
ENCFF000GFU
\end{tabular} & \begin{tabular}{|l} 
bipolar neuron \\
MSC of adipose
\end{tabular} & $\begin{array}{l}\text { 6: Others } \\
\text { character(0) }\end{array}$ & $\begin{array}{l}\text { ENCSR968WKR } \\
\text { ENCSR000CTZ }\end{array}$ & $\begin{array}{l}\text { total RNA-seq } \\
\text { total RNA-seq }\end{array}$ & 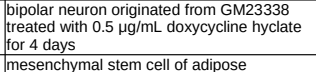 & $\begin{array}{l}\text { Thomas Gingeras, CSHL } \\
\text { Thomas Gingeras, CSHL }\end{array}$ & $\begin{array}{l}\text { ENCODE } \\
\text { ENCODE }\end{array}$ & \begin{tabular}{|l} 
Homo sapiens \\
Homo sapiens
\end{tabular} & \begin{tabular}{|l} 
adult \\
adult
\end{tabular} & $\begin{aligned} 53 \text { year } \\
42,37 \text { year }\end{aligned}$ & doxycycline hyclate \\
\hline \begin{tabular}{|l} 
ENCFFO00GFU \\
ENCFF000GGR \\
\end{tabular} & MSC of adiposese & 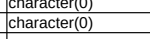 & ENCSROOOCTZ & $\begin{array}{l}\text { totar RNA-Seq } \\
\text { total RNA-seq }\end{array}$ & $\begin{array}{l}\text { mesenchymal stem ceil or aupose } \\
\text { mesenchymal stem cell of adipose }\end{array}$ & $\begin{array}{l}\text { nimomas } \mathrm{Gingeras,} \mathrm{CHL} \\
\text { Thomas Gingeras, CSHL }\end{array}$ & $\begin{array}{l}\text { ENCOE } \\
\text { ENCODE }\end{array}$ & $\begin{array}{l}\text { Homo saplens } \\
\text { Homo sapiens } \\
\end{array}$ & adult & $\begin{array}{l}4.2,3 \text { y year } \\
42,37 \text { year }\end{array}$ & \\
\hline ENCFF001RBG & $\begin{array}{l}\text { lung microvascular } \\
\text { endothelial cell }\end{array}$ & 8: Endothelial cells & ENCSRO00AAP & total RNA-seq & $\begin{array}{l}\text { lung microvascular endothelial cell female } \\
\text { adult (55 years) and male adult }(63 \text { yearss) }\end{array}$ & Thomas Gingeras, CSHL & ENCODE & Homo sapiens & adult & 63,55 year & \\
\hline ENCFF001RBW & $\begin{array}{l}\text { lung microvascular } \\
\text { lendothelial exll }\end{array}$ & 8: Endothelial cells & ENCSROOOAAP & total RNA-seq & 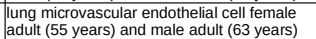 & Thomas Gingeras, CSHL & ENCODE & Homo sapiens & adult & 63,55 year & \\
\hline \begin{tabular}{|l|} 
ENCFF840QXH \\
ENCFF480YLH
\end{tabular} & 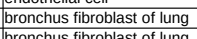 & $\begin{array}{l}\text { 1: Fibroblast } \\
\text { 1. Fibrolast }\end{array}$ & ENCSR620NSN & 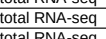 & $\begin{array}{l}\text { bronchus stibroblast of lung } \\
\text { bronchcus fibrobast of lung }\end{array}$ & Thomas Gingeras, CSHL & $\begin{array}{l}\text { ENCODE } \\
\text { ENCDE }\end{array}$ & $\begin{array}{l}\text { Homo sapiens } \\
\end{array}$ & lunknown & unknown & \\
\hline $\begin{array}{l}\text { ENCFF480YLH } \\
\text { ENCFF001RAK }\end{array}$ & bronchus fibroblast of lung & 1: Fibroblast & ENCSR620NSN & toal RNA-Seq & $\begin{array}{l}\text { bronchus fibroblast of lung } \\
\text { tracheal pithelial cell male adult }(21 \text { year) }\end{array}$ & Thomas Gingeras, CSHL & ENCODE & Homo sapiens & unknown & unknown & \\
\hline \begin{tabular}{|l|} 
ENCFOUIRAK \\
ENCFF001RBS
\end{tabular} & racheal epithelial cell & 9: Epithelial cells & ENCSRO00AAR & total RNA-seq & 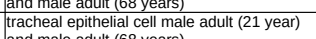 & Thomas Gingeras, CSHL & ENCODE & Homo sapiens & adult & 2,68 year & \\
\hline ENCFF001QZE & 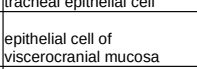 & character(0) & ENCSROOOAAL & total RNA-seq & 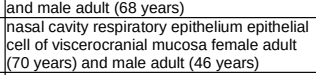 & Thomas Gingeras, CSHL & ENCODE & Homo sapiens & adult & 46,7year & \\
\hline ENCFF001QZI & $\begin{array}{l}\text { lepithelial cell of } \\
\text { viscerocranial mucosa }\end{array}$ & character(0) & ENCSROOOAAL & total RNA-seq & 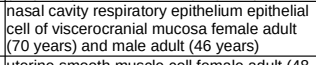 & Thomas Gingeras, CSHL & ENCODE & Homo sapiens & adult & ar & \\
\hline ENCFF001RBA & uterine smooth muscle cell & 7: SMC & ENCSROOOAAV & total RNA-seq & $\begin{array}{l}\text { uterine smooth muscle cell female adult (48 } \\
\text { years) and female adult (50 years) }\end{array}$ & Thomas Gingeras, CSHL & ENCODE & Homo sapiens & adult & 48,5 year & \\
\hline ENCFF001RCC & uterine smooth muscle cell & 7: SMC & ENCSROOOAAV & total RNA-seq & $\begin{array}{l}\text { uterine smooth muscle cell female adult (48 } \\
\text { years) and female adult (50 years) }\end{array}$ & Thomas Gingeras, CSHL & ENCODE & Homo sapiens & adult & 48,5 year & \\
\hline ENCFF001QZQ & aortic SMC & character(0) & ENCSRO0OAAA & total RNA-seq & $\begin{array}{l}\text { aortic smooth muscle cell male adult (21 } \\
\text { year) and male adult (54 years) }\end{array}$ & Thomas Gingeras, CSHL & ENCODE & Homo sapiens & adult & 54,21 year & \\
\hline ENCFF001RAM & aortic SMC & character(0) & ENCSRO0OAAA & total RNA-seq & $\begin{array}{l}\text { aortic smooth muscle cell male adult (21 } \\
\text { year) and male adult (54 years) }\end{array}$ & Thomas Gingeras, CSHL & ENCODE & Homo sapiens & adult & 54,21 year & \\
\hline ENCFFO0OIGA & melanocyte of skin & 6: Others & ENCSROOOCUQ & total RNA-seq & $\begin{array}{l}\text { melanocyte of skin male child (1 year) and } \\
\text { male child ( } 3 \text { years) }\end{array}$ & Thomas Gingeras, CSHL & ENCODE & Homo sapiens & child & 3,1 year & \\
\hline ENCFF000IGU & melanocyte of skin & 6: others & ENCSROOOCUQ & total RNA-seq & $\begin{array}{l}\text { melanocyte of skin male child ( } 1 \text { year) and } \\
\text { male child ( } 3 \text { years) }\end{array}$ & Thomas Gingeras, CSHL & ENCODE & Homo sapiens & child & 3,1 year & \\
\hline ENCFF446LHV & dendritic cell & 6: others & ENCSR022BYE & total RNA-seq & 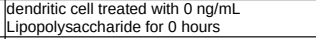 & Manuel Garber, UMass & GGR & Homo sapiens & unknown & unknown & Lipopolysaccharide \\
\hline ENCFF817RIH & dendritic cell & 6: Others & ENCSR227MWL & total RNA-seq & $\begin{array}{l}\text { dendritic cell treated with } 100 \mathrm{ng} / \mathrm{mL} \\
\text { Lipopolysaccharide for } 2 \text { hours }\end{array}$ & Manuel Garber, UMass & GGR & Homo sapiens & unknown & unknown & Lipopolysaccharide \\
\hline ENCFF662HMZ & dendritic cell & 6: Others & ENCSR975YGW & total RNA-seq & $\begin{array}{l}\text { dendrititc cell treated with } 100 \mathrm{ng} / \mathrm{mL} \\
\text { Lipopolysaccharide for } 2 \text { hours }\end{array}$ & Manuel Garber, UMass & GGR & Homo sapiens & unknown & unknown & Lipopolysaccharide \\
\hline ENCFF001RAG & 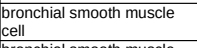 & 7: SMC & ENCSROOOAAE & total RNA-seq & $\begin{array}{l}\text { bronchial smooth muscle cell male adult (52 } \\
\text { years) and male adult (59 years) }\end{array}$ & Thomas Gingeras, CSHL & ENCODE & Homo sapiens & adult & 52,59 year & \\
\hline ENCFF001RAS & \begin{tabular}{|l|l|} 
bronchial smooth muscle \\
cell
\end{tabular} & 7: SMC & ENCSROOOAAE & total RNA-seq & $\begin{array}{l}\text { bronchial smooth muscle cell male adult (52 } \\
\text { years) and male adult (59 years) }\end{array}$ & Thomas Gingeras, CSHL & ENCODE & Homo sapiens & adult & 52,59 year & \\
\hline ENCFF001RAA & $\begin{array}{l}\text { endothelial cell of coronary } \\
\text { artery }\end{array}$ & 8: Endothelial cells & ENCSROOOAAF & total RNA-seq & $\begin{array}{l}\text { endothelial cell of coronary artery female } \\
\text { adult (41 year) and male adult ( } 77 \text { years) }\end{array}$ & Thomas Gingeras, CSHL & ENCODE & Homo sapiens & adult & 41,77 year & \\
\hline ENCFF001RBK & $\begin{array}{l}\text { endotothelial cell of coronary } \\
\text { artery }\end{array}$ & 8: Endothelial cells & ENCSRO0OAAF & total RNA-seq & 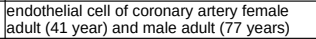 & Thomas Gingeras, CSHL & ENCODE & Homo sapiens & adult & 41,77 year & \\
\hline ENCFF001QZY & \begin{tabular}{|l|} 
smooth muscle cell of the \\
coronary artery
\end{tabular} & 7: SMC & ENCSRO0OAAG & total RNA-seq & $\begin{array}{l}\text { smooth muscle cell of the coronany artery } \\
\text { female adult (53 years) and male adult ( } 55 \\
\text { years) }\end{array}$ & Thomas Gingeras, CSHL & ENCODE & Homo sapiens & adult & 53,5 & \\
\hline ENCFF001RCG & \begin{tabular}{|l|} 
smooth muscle cell of the \\
coronary artery
\end{tabular} & 7: SMC & ENCSROOOAAG & total RNA-seq & $\begin{array}{l}\text { smooth muscle cell of the coronany adtery } \\
\text { female adult (53 years) and male adult ( } 55 \\
\text { years) }\end{array}$ & Thomas Gingeras, CSHL & ENCODE & Homo sapiens & adult & ear & \\
\hline ENCFF001QZU & $\begin{array}{l}\text { pulmonary artery } \\
\text { endothelial cell }\end{array}$ & 8: Endothelial cells & ENCSROOOAAM & total RNA-seq & $\begin{array}{l}\text { pulmonary artery endothelial cell male adult } \\
\text { (23 years) and male adult (52 years) }\end{array}$ & Thomas Gingeras, CSHL & ENCODE & Homo sapiens & adult & 23,52 year & \\
\hline ENCFF001RCE & \begin{tabular}{|l|}
$\begin{array}{l}\text { pulmonary artery } \\
\text { endothelial cell }\end{array}$ \\
\end{tabular} & 8: Endothelial cells & ENCSROOOAAM & total RNA-seq & $\begin{array}{l}\text { pulmonory artery endothelial cell male adult } \\
\text { (23 years) and male adult (52 years) }\end{array}$ & Thomas Gingeras, CSHL & ENCODE & Homo sapiens & adult & 23,52 year & \\
\hline ENCFF071TOB & dendritic cell & 6: others & ENCSR084JIA & total RNA-seq & $\begin{array}{l}\text { dendritic cell treated with } 100 \text { ng/mL } \\
\text { Lipopolysaccharide for } 1 \text { hour }\end{array}$ & Manuel Garber, UMass & GGR & Homo sapiens & unknown & unknown & Lipopolysaccharide \\
\hline ENCFF001RAE & regular cardiac myocyte & 5: Muscle cells & ENCSROOOAAH & total RNA-seq & $\begin{array}{l}\text { regular cardiac myocyte female adult ( } 51 \\
\text { year) and male adult (48 years) }\end{array}$ & Thomas Gingeras, CSHL & ENCODE & Homo sapiens & adult & 48,51|year & \\
\hline ENCFF001RBM & regular cardiac myocyte & 5: Muscle cells & ENCSROOOAAH & total RNA-seq & $\begin{array}{l}\text { regular cardial myouste female adult (51 } \\
\text { leyar and male adult } 488 \text { years) }\end{array}$ & Thomas Gingeras, CSHL & ENCODE & Homo sapiens & adult & 48,51year & \\
\hline ENCFF001RAY & $\begin{array}{l}\text { dermis Iymphatic vessel } \\
\text { endothelial cell }\end{array}$ & 8: Endothelial cells & ENCSROOOAAJ & total RNA-seq & $\begin{array}{l}\text { dermis lymphatic vessel endothelial cell } \\
\text { femala adult (45 years) and male child (6) } \\
\text { years) }\end{array}$ & Thomas Gingeras, CSHL & ENCODE & Homo sapiens & child, adult & 6,45 year & \\
\hline
\end{tabular}


Table_S2 Metadata for ENCODE kmer research

\begin{tabular}{|c|c|c|c|c|c|c|c|c|c|c|c|c|}
\hline ENCFF001RBQ & $\begin{array}{l}\text { dermis Iymphatic vessel } \\
\text { endothelial cell }\end{array}$ & 8: Endothelial cells & ENCSROOOAAJ & total RNA-seq & 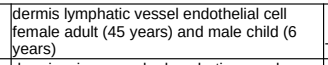 & Thomas Gingeras, CSHL & ENCODE & Homo sapiens & child,adult & & year & \\
\hline ENCFF001RAW & $\begin{array}{l}\begin{array}{l}\text { dermis microvascular } \\
\text { ymmphatic vessel endothelial } \\
\text { celll }\end{array} \\
\end{array}$ & 8: Endothelial cells & ENCSRO0OAAK & total RNA-seq & $\begin{array}{l}\text { dermis microvascular Iymphatic vessel } \\
\text { endotothelial cell temala adult (38 years) and } \\
\text { female adult (64 years) }\end{array}$ & Thomas Gingeras, CSHL & ENCODE & Homo sapiens & adult & 38,6 & year & \\
\hline ENCFF001RBE & $\begin{array}{l}\begin{array}{l}\text { dermis microvascular } \\
\text { ymmhatic vessel endothelial } \\
\text { cell }\end{array} \\
\end{array}$ & 8: Endothelial cells & ENCSROOOAAK & total RNA-seq & 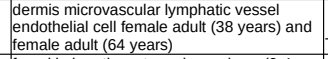 & Thomas Gingeras, CSHL & ENCODE & Homo sapiens & adult & 38. & year & \\
\hline ENCFF $365 \mathrm{NAH}$ & foreskin keratinocyte & 6: Others & ENCSR527SSD & total RNA-seq & $\begin{array}{l}\text { foresskik keratitocyte male newborm (2-4 } \\
\text { days) treated with } 1.2 \mathrm{~mm} \text { calcium for } 5.5 \\
\text { days }\end{array}$ & Michael Snyder, Stanford & GGR & Homo sapiens & newborn & $2-4$ & day & calcium \\
\hline ENCFF668JVG & foreskin keratinocyte & 6: others & ENCSR527SSD & total RNA-seq & $\begin{array}{l}\text { foreskin keratinocyte male newborm (2-4 } \\
\text { days) treated with } 1.2 \mathrm{mM} \text { calcium for } 5.5 \\
\text { days } \\
\text { dendritic }\end{array}$ & Michael Snyder, Stantord & GGR & Homo sapiens & newborn & $2-4$ & day & calcium \\
\hline ENCFF649ASL & dendritic cell & 6: Others & ENCSR571IUZ & total RNA-seq & $\begin{array}{l}\text { dendiritic cell treated with } 100 \mathrm{ng} / \mathrm{mL} \\
\text { Lipopolysaccharide for } 4 \text { hours }\end{array}$ & Manuel Garber, UMass & GGR & Homo sapiens & unknown & unknown & & Lipopolysaccharide \\
\hline ENCFF123JND & dendritic cell & 6: Others & ENCSR760INS & total RNA-seq & $\begin{array}{l}\text { dendriticic cell treated with } 100 \mathrm{ng} / \mathrm{mL} \\
\text { Lippopolysaccharide for } 6 \text { hours }\end{array}$ & Manuel Garber, UMass & GGR & Homo sapiens & unknown & unknown & & Lipopolysaccharide \\
\hline ENCFF945OFS & dendritic cell & 6: Others & ENCSR670MXT & total RNA-seq & $\begin{array}{l}\text { denditicic cell treated with 100 ng/mL } \\
\text { Lipopolysaccharide for } 2 \text { hours }\end{array}$ & Manuel Garber, UMass & GGR & Homo sapiens & unknown & unknown & & Lipopolysaccharide \\
\hline ENCFF124RBL & dendritic cell & 6: Others & ENCSR476TKU & total RNA-seq & $\begin{array}{l}\text { dendititic cell treated with } 100 \mathrm{ng} / \mathrm{mL} \\
\text { Lipopolysacharide for } 4 \text { hours }\end{array}$ & Manuel Garber, UMass & GGR & Homo sapiens & unknown & unknown & & Lipopolysaccharide \\
\hline ENCFF365UNT & dendritic cell & 6: Others & ENCSR475TVN & total RNA-seq & $\begin{array}{l}\text { dendritic cell treated with } 100 \mathrm{ng} / \mathrm{mL} \\
\text { Lipopolysaccharide for } 6 \text { hours }\end{array}$ & Manuel Garber, UMass & GGR & Homo sapiens & unknown & unknown & & Lipopolysaccharide \\
\hline ENCFF658PGS & dendritic cell & 6: Others & ENCSR707JVU & total RNA-seq & $\begin{array}{l}\text { dendititic cell treated with } 100 \mathrm{ng} / \mathrm{mL} \\
\text { Lipopolysaccharide for } 4 \text { hours }\end{array}$ & Manuel Garber, UMass & GGR & Homo sapiens & unknown & unknown & & Lipopolysaccharide \\
\hline ENCFF609HPU & dendritic cell & 6: Others & ENCSR916YUR & total RNA-seq & $\begin{array}{l}\text { dendiritic cell treated with } 100 \mathrm{ng} / \mathrm{mL} \\
\text { Lipopolysaccharide for } 6 \text { hours }\end{array}$ & Manuel Garber, UMass & GGR & Homo sapiens & unknown & unknown & & Lipopolysaccharide \\
\hline ENCFF000GLZ & placental epithelial cell & 9: Epithelial cells & ENCSROOOCUP & total RNA-seq & $\begin{array}{l}\begin{array}{l}\text { placectala epithelial cell female newbor } \\
\text { male newborm }\end{array} \\
\end{array}$ & Thomas Gingeras, CSHL & ENCODE & Homo sapiens & newborn & unknown & & \\
\hline ENCFFOOOGMA & placental epithelial cell & 9: Epithelial cells & ENCSROOOCUP & total RNA-seq & $\begin{array}{l}\begin{array}{l}\text { placental epithelial cell female newborn and } \\
\text { male newborn }\end{array} \\
\end{array}$ & Thomas Gingeras, CSHL & ENCODE & Homo sapiens & newborn & unknown & & \\
\hline ENCFF000GYE & subcutaneous preadipocyte & 4: Progenitor or Stem cell & ENCSROOOCUM & total RNA-seq & $\begin{array}{l}\text { subcutaneous preadipocyte female adult ( (6) } \\
\text { years) and male adult }(65 \text { years) }\end{array}$ & Thomas Gingeras, CSHL & ENCODE & Homo sapiens & adult & 65,62 & year & \\
\hline ENCFF000GZD & subcutaneous preadipocyte & 4: Progenitor or Stem cell & ENCSROOOCUM & total RNA-seq & $\begin{array}{l}\begin{array}{l}\text { subcutaneous preadipocyte female adl } \\
\text { years) and male adult (65 years) }\end{array} \\
\end{array}$ & Thomas Gingeras, CSHL & ENCODE & Homo sapiens & adult & $65,62 y$ & year & \\
\hline ENCFF947BAG & dendritic cell & 6: Others & ENCSR146PLL & total RNA-seq & $\begin{array}{l}\text { dendiritic cell treated with } 100 \mathrm{ng} / \mathrm{mL} \\
\text { Lipopolysaccharide for } 4 \text { hours }\end{array}$ & Manuel Garber, UMass & GGR & Homo sapiens & unknown & unknown & & Lipopolysaccharide \\
\hline ENCFF659EDN & $\begin{array}{l}\text { mammary microvascular } \\
\text { endothelial cell }\end{array}$ & 8: Endothelial cells & ENCSR815UVL & total RNA-seq & $\begin{array}{l}\text { mammary microvascular endothelial cell } \\
\text { female adult (26 years) }\end{array}$ & Thomas Gingeras, CSHL & ENCODE & Homo sapiens & adult & & year & \\
\hline ENCFF143UMB & $\begin{array}{l}\text { mammary microvascular } \\
\text { endothelial cell }\end{array}$ & 8: Endothelial cells & ENCSR815UVL & total RNA-seq & $\begin{array}{l}\text { mammary microvascular endothelial cell } \\
\text { female adult (26 years) }\end{array}$ & Thomas Gingeras, CSHL & ENCODE & Homo sapiens & adult & & year & \\
\hline ENCFF001QZW & $\begin{array}{l}\text { epithelial cell of umbilical } \\
\text { artery }\end{array}$ & 9: Epithelial cells & ENCSROOOAAT & total RNA-seq & $\begin{array}{l}\text { epithelial cell of umbilical artery female } \\
\text { newborn and male newborn }\end{array}$ & Thomas Gingeras, CSHL & ENCODE & \begin{tabular}{|l} 
Homo sapiens \\
\end{tabular} & newborn & unknown & & \\
\hline ENCFF001RBC & $\begin{array}{l}\text { epithelial cell of umbilical } \\
\text { artery }\end{array}$ & 9: Epithelial cells & ENCSROOOAAT & total RNA-seq & $\begin{array}{l}\text { epithelial cell of umbilical artery female } \\
\text { newborn and male newborn }\end{array}$ & Thomas Gingeras, CSHL & ENCODE & Homo sapiens & newborn & unknown & & \\
\hline ENCFF001RBU & $\begin{array}{l}\text { smooth muscle cell of the } \\
\text { umbilical artery }\end{array}$ & 7: SMC & ENCSROOOAAU & total RNA-seq & $\begin{array}{l}\text { smooth muscle cell of the umbilical artery } \\
\text { female newborn and male newborn }\end{array}$ & Thomas Gingeras, CSHL & ENCODE & Homo sapiens & newborn & unknown & & \\
\hline ENCFF001RBY & $\begin{array}{l}\text { smooth muscle cell of the } \\
\text { umbilical artery }\end{array}$ & 7: SMC & ENCSROOOAAU & total RNA-seq & $\begin{array}{l}\text { smooth muscle cell of the umbilical artery } \\
\text { female newborn and male newborn }\end{array}$ & Thomas Gingeras, CSHL & ENCODE & Homo sapiens & newborn & unknown & & \\
\hline $\begin{array}{l}\text { ENCFF002DMJ } \\
\text { ENCFF002DMP }\end{array}$ & \begin{tabular}{|l|} 
H7-hESCC \\
H7-hESC \\
\end{tabular} & $\begin{array}{l}\text { 4: Progenitor or Stem cell } \\
\text { 4: Progenitor or Stem celll }\end{array}$ & $\begin{array}{l}\text { ENCSR490SQH } \\
\text { ENCSR49SOH }\end{array}$ & $\begin{array}{l}\text { total RNA-seq } \\
\text { total RA-seg }\end{array}$ & $\begin{array}{l}\text { H7-hESC } \\
\text { H7-hESC }\end{array}$ & $\begin{array}{l}\text { Thomas Gingeras, CSHL } \\
\text { Theme }\end{array}$ & $\begin{array}{l}\text { ENCODE } \\
\text { ENCODE }\end{array}$ & \begin{tabular}{|l|} 
Homo sapiens \\
Homo sapiens \\
\end{tabular} & $\begin{array}{l}\text { embryonic } \\
\text { embronic }\end{array}$ & 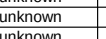 & & \\
\hline ENCFF000IHC & melanocyte of skin & 6: Others & ENCSROOOCUR & total RNA-seq & $\begin{array}{l}\text { m-nelanocyte of skin female adult (52 years) } \\
\text { and male adult (55 years) }\end{array}$ & Thomas Gingerar, & ENCODE & $\begin{array}{l}\text { Homo Sapiens } \\
\text { Homo sapiens } \\
\end{array}$ & adult & unknown & year & \\
\hline ENCFFO0OIHY & melanocyte of skin & 6: Others & ENCSRO0OCUR & total RNA-seq & $\begin{array}{l}\text { melanocyte of skin female adult ( } 52 \text { years) } \\
\text { and male adult (55 years) }\end{array}$ & Thomas Gingeras, CSHL & ENCODE & Homo sapiens & adult & $55,52 y$ & year & \\
\hline ENCFF575SLU & cardiac ventricle fibroblast & 1: Fibroblast & ENCSR369RVN & total RNA-seq & $\begin{array}{l}\text { cardiac ventricile fibroblast NONE and male } \\
\text { adutt (18 years }\end{array}$ & Thomas Gingeras, CSHL & ENCODE & Homo sapiens & adult,unknown & 18,unknown & year & \\
\hline ENCFF157UXG & cardiac ventricle fibroblast & 1: Fibroblast & ENCSR369RVN & total RNA-seq & $\begin{array}{l}\text { cardiac venticle fibroblast NONE and male } \\
\text { adult (18years) }\end{array}$ & Thomas Gingeras, CSHL & ENCODE & Homo sapiens & adult,unknown & 18,unknown & year & \\
\hline ENCFF689YVG & pericardium fibroblast & 1: Fibroblast & ENCSR362HMX & total RNA-seq & $\begin{array}{l}\text { pericardium fibroblast NONE and female } \\
\text { embryo (20 weeks) }\end{array}$ & Thomas Gingeras, CSHL & ENCODE & Homo sapiens & embryonic,unkn & 20,unknown n & week & \\
\hline ENCFF005EEW & pericardium fibroblast & 1: Fibroblast & ENCSR362HMX & total RNA-seq & $\begin{array}{l}\begin{array}{l}\text { pericardium fibroblast NONE and female } \\
\text { embryo (20 weeks) }\end{array} \\
\end{array}$ & Thomas Gingeras, CSHL & ENCODE & Homo sapiens & embryonic,unkn & 20,unknown n & week & \\
\hline ENCFF746KSI & $\begin{array}{l}\text { epithelial cell of alveolus of } \\
\text { lung }\end{array}$ & 9: Epithelial cells & ENCSR897KTO & total RNA-seq & $\begin{array}{l}\text { epithelial cell of alveolus of lung NONE and } \\
\text { female embryo (21 week) }\end{array}$ & Thomas Gingeras, CSHL & ENCODE & Homo sapiens & unknown,embry & unknown,21 & week & \\
\hline ENCFF408FWI & $\begin{array}{l}\text { epithelial cell of alveolus of } \\
\text { lung }\end{array}$ & 9: Epithelial cells & ENCSR897KTO & total RNA-seq & $\begin{array}{l}\text { epithelial cell of alveolus of lung NONE and } \\
\text { female embryo (21 week) }\end{array}$ & Thomas Gingeras, CSHL & ENCODE & Homo sapiens & unknown,embry & unknown, 21 & week & \\
\hline ENCFF109IUU & kidney epithelial cell & 9: Epithelial cells & ENCSR373BDG & total RNA-seq & $\begin{array}{l}\text { lemidey enithelialal cell mala embryo (22) } \\
\text { weeks) and male newborn }\end{array}$ & Thomas Gingeras, CSHL & ENCODE & Homo sapiens & embryonic,new & 22,unknown & week & \\
\hline ENCFF999ZER & kidney epithelial cell & 9: Epithelial cells & ENCSR373BDG & total RNA-seq & $\begin{array}{l}\text { kidney epithelial cell male embryo (22 } \\
\text { weeks) and male newborn }\end{array}$ & Thomas Gingeras, CSHL & ENCODE & Homo sapiens & embryonic,new & 22, unknown n & week & \\
\hline ENCFF353STY & cardiac atrium fibroblast & 1: Fibroblast & ENCSR110BDY & total RNA-seq & 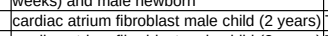 & Thomas Gingeras, CSHL & ENCODE & $\begin{array}{l}\text { Homo sapiens } \\
\end{array}$ & & & 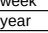 & \\
\hline ENCFF753GPU & cardiac atrium fibroblast & 1: Fibroblast & ENCSR110BDY & total RNA-seq & 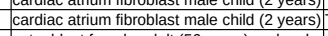 & Thomas Gingeras, CSHL & ENCODE & Homo sapiens & child & & year & \\
\hline ENCFF000GJC & osteoblast & 3: Osteo/Chondro lineage & ENCSROOOCUF & total RNA-seq & $\begin{array}{l}\text { osteoblasst temale adult (56 years) and male } \\
\text { adult } 6 \text { y years) }\end{array}$ & Thomas Gingeras, CSHL & ENCODE & Homo sapiens & adult & $56,62 y$ & year & \\
\hline ENCFF000GKC & osteoblast & 3: Osteo/Chondro lineage & ENCSROOOCUF & total RNA-seq & $\begin{array}{l}\text { osteoblasst female adult (56 years) and male } \\
\text { adult (62 years) }\end{array}$ & Thomas Gingeras, CSHL & ENCODE & Homo sapiens & adult & $56,62 y$ & year & \\
\hline ENCFF000GET & mammary epithelial cell & 9: Epithelial cells & ENCSROOOCUN & total RNA-seq & $\begin{array}{l}\text { mammary epithelial cell female adult (23 } \\
\text { years) }\end{array}$ & Thomas Gingeras, CSHL & ENCODE & Homo sapiens & adult & & year & \\
\hline $\begin{array}{l}\text { ENCFF353SSPR } \\
\text { ENCFFO30WXU }\end{array}$ & cardiac muscle celll & 5: Muscle cells & ENCSR379YAE & total RNA-seq & cardiac muscle cell originated from RUES2 & Thomas Gingeras, CSHL & ENCODE & Homo sapiens & embryonic & unknown & & \\
\hline ENCFF472OXK & cardiac muscle cell & 5: Muscle cells & ENCSR379YAE & total RNA-seq & $\begin{array}{l}\text { cardiac muscle cell originated from RUES2 } \\
\text { foreskin keratincotye mala newborm (2-4. } \\
\text { days) treated with } 1.2 \text { mM calcium for } 2.5 \\
\text { days }\end{array}$ & \begin{tabular}{|l} 
Thomas Gingeras, CSHL \\
Michael Snyder, Stanford
\end{tabular} & ENCODE & Homo sapiens & embryonic & unknown & day & \\
\hline & & 6: Others & ENCSR959LTT & al RNA-seg & $\begin{array}{l}\text { toreskin keratinocyte male newborm (2-4 } \\
\text { days) treated with } 1.2 \mathrm{mM} \text { calcium for } 2.5 \\
\text { days }\end{array}$ & Michael Snyder, Stanford & GGR & Homo sapiens & newborm & & day & alcium \\
\hline
\end{tabular}


Table_S2 Metadata for ENCODE kmer research

\begin{tabular}{|c|c|c|c|c|c|c|c|c|c|c|c|}
\hline $\begin{array}{l}\text { ENCFFO0OGIC } \\
\text { ENCFFO0OGIV }\end{array}$ & \begin{tabular}{|l} 
MSC of Wharton's jelly \\
MSC of Wharton's jelly
\end{tabular} & $\begin{array}{l}\text { character(0) } \\
\text { character(0) }\end{array}$ & $\begin{array}{l}\text { ENCSRO0OCUO } \\
\text { ENCSROOOCUO }\end{array}$ & $\begin{array}{l}\text { total RNA-seq } \\
\text { total RNA-seq }\end{array}$ & $\begin{array}{l}\text { Imesenchymal stem cell of Wharton's jelly } \\
\text { mesenchymal stem cell of Wharton's jelly }\end{array}$ & $\begin{array}{l}\frac{\text { Thomas Gingeras, CSHL }}{\text { Thomas Gingeras, CSHL }} \\
\end{array}$ & $\begin{array}{l}\text { ENCODE } \\
\text { ENCODE }\end{array}$ & $\begin{array}{l}\text { Homo sapiens } \\
\text { Homo sapiens } \\
\end{array}$ & $\begin{array}{l}\text { newborm } \\
\text { newborn }\end{array}$ & $\begin{array}{l}\text { unknown } \\
\text { unknown }\end{array}$ & \\
\hline $\begin{array}{l}\text { ENCFF548JWS } \\
\text { ENCEF }\end{array}$ & $\begin{array}{l}\text { smooth muscle cell } \\
\text { smmothmuscle cell }\end{array}$ & 7: SMC & $\begin{array}{l}\text { ENCSR052FJA } \\
\text { ENCS05FIA }\end{array}$ & $\begin{array}{l}\text { total RNA-seq } \\
\text { totat RNA }- \text { seg }\end{array}$ & $\begin{array}{l}\text { smooth muscle cell originated from H99 } \\
\text { smooth muscle cell originated trom H9 }\end{array}$ & 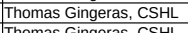 & $\begin{array}{ll}\text { ENCODE } \\
\text { FNCDEF }\end{array}$ & $\begin{array}{ll}\text { Homo sapiens } \\
\text { Hom sanins }\end{array}$ & embryonic & & 型 \\
\hline & & & & & 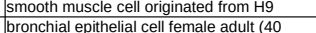 & & & Homo sapiens & & & \\
\hline ENCFF001RAC & bronchial epithelial cell & 9: Epithelial cells & ENCSROOOAAD & total RNA-seq & 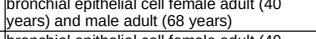 & Thomas Gingeras, CSHL & ENCODE & Homo sapiens & adult & & ear \\
\hline ENCFFO01RBO & bronchial epithelial cell & 9: Epithelial cells & ENCSROOOAAD & total RNA-seq & 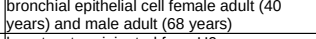 & Thomas Gingeras, CSHL & ENCODE & Homo sapiens & adult & & year \\
\hline & $\begin{array}{l}\text { hepatocyte } \\
\text { hepatocyte }\end{array}$ & $\begin{array}{l}\text { 6: Others } \\
\text { 6: Others }\end{array}$ & \begin{tabular}{|l} 
ENCSR908ZAS \\
ENCSR908ZAS
\end{tabular} & $\begin{array}{l}\text { total INAA-seq } \\
\text { total RNAseg }\end{array}$ & 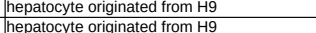 & $\begin{array}{l}\text { Thomas Gingeras, CSHL } \\
\text { Thomas Gingeras CSHL }\end{array}$ & ENCODE & Homo sapiens & $\begin{array}{l}\text { embryonic } \\
\text { embropic }\end{array}$ & & lay \\
\hline ENCFF996KMK & neural progenitor cell & 4: Progenitor or Stem cell & ENCSR244ISQ & $\begin{array}{l}\text { total RNAA-seq } \\
\text { tota-seq }\end{array}$ & 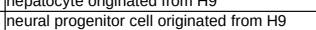 & $\begin{array}{l}\text { Thomas Gingeras, CSHL } \\
\text { Thomas Gingeras, CSHL }\end{array}$ & $\begin{array}{l}\text { ENCUE } \\
\text { ENCODE }\end{array}$ & $\begin{array}{l}\text { Homm sapiens } \\
\text { Homo sapiens } \\
\end{array}$ & $\begin{array}{l}\text { Eembiyyonic } \\
\text { embryonic }\end{array}$ & & $\frac{a y}{a y}$ \\
\hline ENCFF939FVE & neural progenitor cell & 4: Progenitor or Stem cell & & total RNA-seq & neural progenitor cell originated from $\mathrm{H} 9$ & Thomas Gingeras, CSHL & & Homo sapiens & embryonic & & 焉 \\
\hline ENCFF002DMH & skeletal muscle myoblast & 5: Muscle cells & ENCSR444WHQ & total RNA-seq & skeletal muscle myoblast & Thomas Gingeras, CSHL & ENCODE & Homo sapiens & unknown & unknown & \\
\hline ENCFF002DML & skeletal muscle myoblast & 5: Muscle cells & ENCSR444WHQ & total RNA-seq & skeletal muscle myoblast & Thomas Gingeras, CSHL & ENCODE & Homo sapiens & unknown & unknown & \\
\hline ENCFF119TIN & myocyte & 5: Muscle cells & ENCSR894WMQ & fotal RNA-seq & myocyte originated from LHCN-M2 & Thomas Gingeras, CSHL & ENCODE & Homo sapiens & adult & & \\
\hline $\begin{array}{l}\text { ENCFFFO0OUG } \\
\text { ENCFOOGGHA }\end{array}$ & $\begin{array}{l}\text { myocyte } \\
\text { mysc of the bone marrow }\end{array}$ & $\begin{array}{l}\text { 5: Muscle cells } \\
\text { character(0) }\end{array}$ & $\begin{array}{l}\text { ENCSR894WMQ } \\
\text { ENCSRO00CD }\end{array}$ & totat RNA-seq & myocyte originated from LHCN-M2 & Thomas Gingeras, CSHL & $\begin{array}{l}\text { ENCODE } \\
\text { ENCDE }\end{array}$ & Homo sapiens & adult & & \\
\hline $\begin{array}{l}\text { ENCFOOUOGA } \\
\text { ENCFFOOOGHX }\end{array}$ & 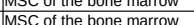 & 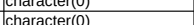 & $\begin{array}{l}\text { ENCSROUCUD } \\
\text { ENCSROOOCUD }\end{array}$ & 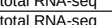 & 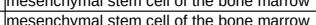 & 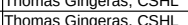 & 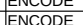 & 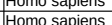 & 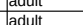 & & \\
\hline ENCFF464PFE & airway epithelial cell & 9: Epithelial cells & ENCSR822SUG & total RNA-seq & aimay epithelial cell & Thomas Gingeras, CSHL & ENCODE & Homo sapiens & unknown & & \\
\hline ENCFF846YCY & alirway epunthenarcent & 9: Epithelial cells & ENCSR822SUG & total RNA-seq & aimay epithelial cell & Thomas Gingeras, CSHL & ENCODE & Homo sapiens & unknown & unknown & \\
\hline ENCFF541NUM & $\begin{array}{l}\text { eepithelial cell of proximal } \\
\text { tutuble }\end{array}$ & 9: Epithelial cells & ENCSR118TVR & total RNA-seq & epithelial cell of proximal tubule & Thomas Gingeras, CSHL & ENCODE & Homo sapiens & unknown & unknown & \\
\hline ENCFF479JTM & $\begin{array}{l}\text { epithelial cell of proximal } \\
\text { tubule }\end{array}$ & 9: Epithelial cells & ENCSR118TVR & total RNA-seq & epithelial cell of proximal tubule & Thomas Gingeras, CSHL & ENCODE & Homo sapiens & unknown & unknown & \\
\hline ENCFF000FJX & $\begin{array}{l}\text { fibroblast of the aortic } \\
\text { adventitia }\end{array}$ & 1: Fibroblast & ENCSRO0OCUJ & total RNA-seq & $\begin{array}{l}\text { fibroblast of the aortic adventitia female } \\
\text { adult (24 years) and male adult (47 yearss) }\end{array}$ & Thomas Gingeras, CSHL & ENCODE & Homo sapiens & adult & $47,2$. & ear \\
\hline ENCFFO0OFKI & $\begin{array}{l}\text { fibiboblast of the aortic } \\
\text { adventitia }\end{array}$ & 1: Fibroblast & ENCSROoocuj & total RNA-seq & $\begin{array}{l}\text { fibroblast of the aortic avdventitita female } \\
\text { adult (24 years) and male adult (47 years) }\end{array}$ & Thomas Gingeras, CSHL & ENCODE & Homo sapiens & adult & 47,2 & year \\
\hline ENCFFOOOEUS & $\begin{array}{l}\text { hemamatopoietic multipotent } \\
\text { progenitor cell }\end{array}$ & 4: Progenitor or Stem cell & NCSR000CUA & total & hematopoietic multipotent progenitor cell & Thom: & ENC & apiens & unknown & unknown & \\
\hline $\begin{array}{l}\text { ENCFF } 393 G Q Z \\
\text { ENCEF2ADO }\end{array}$ & $\begin{array}{l}\text { astrocyte } \\
\text { astrocyte. }\end{array}$ & $\begin{array}{l}\text { 6: Others } \\
\text { 6. : thers }\end{array}$ & \begin{tabular}{|l|l|} 
ENCSR233IJT \\
ENCS233IT
\end{tabular} & $\begin{array}{l}\text { total RNA-seq } \\
\text { totat RNA }\end{array}$ & $\begin{array}{l}\text { astrocyte } \\
\text { astrocyte }\end{array}$ & $\begin{array}{l}\text { Thomas Gingeras, CSHL } \\
\text { Themas Gineras CSHL }\end{array}$ & $\begin{array}{l}\text { ENCODE } \\
\text { ENCDE }\end{array}$ & Homo sapiens & unknown & unknown & \\
\hline ENCFF424DEO & astrocyte & 6: Others & & & lung fe & & ENCODE & Homo sapiens & Junkrowne & unknown & \\
\hline ENCFF001QZO & fibroblast of lung & 1: Fibroblast & ENCSROOOAAO & total RNA-seq & and male adult (23 years) & Thomas Gingeras, CSHL & ENCODE & Homo sapiens & adult & & ar \\
\hline ENCFF001RBI & fibroblast of lung & 1: Fibroblast & ENCSROOOAAO & total RNA-seq & $\begin{array}{l}\text { fibrobbast of lung female add } \\
\text { and male adult (23 years) }\end{array}$ & Thomas Gingeras, CSHL & ENCODE & Homo sapiens & adult & 83,2 & year \\
\hline
\end{tabular}


Table_S3 Meatadata for single cell analysis and FANTOM6 kmer research

\begin{tabular}{|c|c|c|c|c|c|c|c|c|c|c|c|c|c|}
\hline In ID & 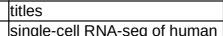 & experiment type & fraction & run_no & target_gene_symbol & specie & $\frac{\text { study _title }}{\text { single-cellt }}$ & Study ID & reads & read_length & library_selection & perturb_id & Ceel_type_al \\
\hline SRR7294023 & 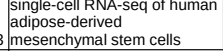 & $\begin{array}{l}10 \text { genomics } \\
\text { sequencing }\end{array}$ & NA & NA & NA & & 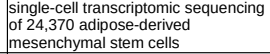 & PRJNA472816 & 17654510411 & 149.75 & PolyA & NA & Ad-MSCs \\
\hline SRR7253635 & $\begin{array}{l}\text { single-cell RNA-seq of human } \\
\text { adipose-derived } \\
\text { meseschymal stem cells }\end{array}$ & $\begin{array}{l}\text { 10X genomics } \\
\text { sequencing }\end{array}$ & NA & NA & NA & Homo sapiens & 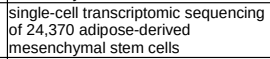 & PRJNA472816 & 26571953 & 49.99 & PolyA & NA & d-Mscs \\
\hline \begin{tabular}{|l|} 
SRR7363187 \\
Run ID
\end{tabular} & $\begin{array}{l}\text { single-cell RNA-seq of human } \\
\text { adipose-derived } \\
\text { mesenchymal stem cells } \\
\text { titles }\end{array}$ & \begin{tabular}{|l} 
10x genomics \\
sequencing \\
experiment_type
\end{tabular} & NA & \begin{tabular}{|l|} 
NA \\
run_no
\end{tabular} & \begin{tabular}{|l} 
NA \\
target___
\end{tabular} & & $\begin{array}{l}\text { single-cell transcriptomic sequencing } \\
\text { of 24,370 odipose-derived } \\
\text { mesenchymal stem cells } \\
\text { study_title }\end{array}$ & \begin{tabular}{|l} 
PRJNA472816 \\
Study ID
\end{tabular} & $\begin{array}{l}3528735371 \\
\text { reads }\end{array}$ & $\frac{149.98}{\text { read length }}$ & $\begin{array}{l}\text { PolyA } \\
\text { library selection }\end{array}$ & $\frac{N A}{\text { perturb id }}$ & $\frac{\text { Ad-MSCs }}{\text { cell trpe alit }}$ \\
\hline DRR177156 & $\begin{array}{l}\text { RDhi10602_ATCACG.experi } \\
\text { ment }\end{array}$ & Reference & cytoplasmic & & ONA & Homo sapiens & FANTOM6 project & PRJDB7993 & 33503637 & & 51 Inverse rRNA & NA & HDF (neonat \\
\hline DRR177155 & $\begin{array}{l}R \text { Ront } \\
\text { ent }\end{array}$ & Reference & cytoplasmic & & ONA & Homo sapiens & FANTOM6 project & PRJDB7993 & 30096528 & & 51 |Inverse rRNA & $\mathrm{NA}$ & HDF (neon \\
\hline DRR177154 & $\begin{array}{l}\text { RDhilo } \\
\text { ment }\end{array}$ & Reference & nuclear & & $0 \mid N A$ & Homo sapiens & FANTOM6 project & PRJDB7993 & 35912547 & & 51 Inverse rRNA & $\mathrm{NA}$ & HDF (neon \\
\hline DRR177153 & $\begin{array}{l}\text { RDhiloc } \\
\text { ment }\end{array}$ & Reference & nuclear & & ONA & Homo sapiens & FANTOM6 project & PRJDB7993 & 35267146 & & 51 Inverse rRNA & NA & HDF (neon \\
\hline DRR177152 & $\begin{array}{l}\text { RDhil00600_ATCACG.experi } \\
\text { ment }\end{array}$ & Reference & chromatin & & ONA & Homo sapiens & FANTOM6 project & PRJDB7993 & 33268855 & & 51 1. Inverse rRNA & $\mathrm{NA}$ & HDF (neona \\
\hline DRR177151 & & Reference & chromatin & & ONA & Homo sapiens & FANTOM6 project & PRJDB7993 & & & 51|Inverse rRNA & $\mathrm{NA}$ & \\
\hline $\begin{array}{ll}\text { DRR176801 } \\
\text { DRP17600 }\end{array}$ & CNhi10765_ACC.experiment & geted & $N A$ & & 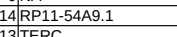 & Homo sapiens & & $\begin{array}{l}\text { PRJDB7993 } \\
\end{array}$ & 177143511 & & CAGE & & HDF (neona \\
\hline $\begin{array}{l}\text { DRR17600 } \\
\text { DRR176799 }\end{array}$ & 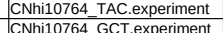 & \begin{tabular}{|l} 
Targeted \\
Negative co
\end{tabular} & NA & & $\begin{array}{l}13 \text { 13ERC } \\
14 \text { NA }\end{array}$ & $\begin{array}{l}\text { Homo sapiens } \\
\text { Homo saiens }\end{array}$ & $\begin{array}{l}\text { FANTOM6 project } \\
\text { FANTOM6 proiect }\end{array}$ & $\begin{array}{l}\text { PRJDB79993 } \\
\text { PRJBD7993 }\end{array}$ & \begin{tabular}{|l|}
22288472 \\
16779927
\end{tabular} & & $\begin{array}{l}717 \text { CAEE } \\
77 \text { CAGE }\end{array}$ & 41_AD_10 & $\begin{array}{l}\mathrm{HDF} \text { (neonat } \\
\mathrm{HDF} \text { (neonat }\end{array}$ \\
\hline DRR176798 & CNhi10764_GCG.experiment & $\begin{array}{l}\text { Tevaluet } \\
\text { Targeted }\end{array}$ & NA & & 14 RP5-886K2.3 & Homo sapiens & FANTOM6 project & PRJDB7993 & 13412787 & & 7 CAGE & ASO_G023681 & HDF (neonal \\
\hline \begin{tabular}{|l|l|l|l|l} 
DRR176797 \\
\end{tabular} & CNhi10764_CAC.experiment & Targeted & $N A$ & & 14RP5-886K2.3 & Homo sapiens & FANTOM6 project & PRJDB7993 & 13768161 & & 17CAGE & ASO_G0236810_AD_02 & HDF (neona \\
\hline DRR176796 & CNhi10764_ATG.experiment & $\begin{array}{l}\text { Experiment } \\
\text { control }\end{array}$ & NA & & 13 MALAT1 & Homo sapiens & FANTOM6 project & PRJDB7993 & 18335819 & & 17 CAGE & ASO_MALAT1 & \\
\hline \begin{tabular}{|l|l|} 
DRR176731 \\
DRR176730
\end{tabular} & 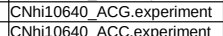 & $\begin{array}{l}\text { Targeted } \\
\text { Targeted }\end{array}$ & NA & & $\begin{array}{l}12 \text { DNM3OS } \\
12 \text { EM2OS }\end{array}$ & $\begin{array}{l}\text { Homo sapiens } \\
\text { Homo sapiens }\end{array}$ & \begin{tabular}{|l} 
FANTOM6 project \\
FANTOM6 proiect
\end{tabular} & $\begin{array}{l}\text { PRJDB79993 } \\
\text { PRJDP7939 }\end{array}$ & \begin{tabular}{|l|l|}
14547994 \\
16086917
\end{tabular} & & $\begin{array}{l}71 \text { CAGE } \\
77 \text { CAGE }\end{array}$ & $\begin{array}{l}\text { ASO_G0230630_AD_04 } \\
\text { ASO G029847 AD } 04\end{array}$ & $\begin{array}{l}\text { HDF (neonat } \\
\text { HDF (neonat }\end{array}$ \\
\hline DPR1767 & Crbingsa & $\begin{array}{l}\text { Experimen } \\
\text { cotron }\end{array}$ & NA & & The & Home saniens & EANTOM & PR1087993 & & & A & & \\
\hline & & & & & & & & & & & & & \\
\hline DRR176727 & CNhi106399GCG.epperiment & Targeted & $\mathrm{NA}$ & & $\frac{12 \text { C110rf95 }}{12 \text { C11 }}$ & Homo sapiens & FANTOM6 project & PRJDB7993 & 12726968 & & 17CAGE & ASO_G0188 & HDF (neon \\
\hline $\begin{array}{ll}\text { DRR176726 } \\
\text { DPD176725 }\end{array}$ & CNhi10639_CAC.experiment & Targeted & $N A$ & & $\begin{array}{l}12 \text { LINC00339 } \\
1 \text { TITSC }\end{array}$ & Homo sapiens & FANTOM6 project & $\begin{array}{l}\text { PRJDB79933 } \\
\text { PDID7002 }\end{array}$ & $\begin{array}{l}13664802 \\
150702\end{array}$ & & $\begin{array}{ll}17 \text { CAGE } \\
7 \text { CACE }\end{array}$ & ASO_G0218510_AD_03 & HDF (neonal \\
\hline $\mathrm{DRF}$ & CNhi10639 & Targrgeted & NA & & 12T TERC & Homo sapiens & jproject & & $\begin{array}{l}12597402 \\
\end{array}$ & & 17 CAGE & ASO_G0270141_AD_10 & HDF (neonat) \\
\hline $\begin{array}{ll}\text { DRR176724 } \\
\text { DRP176659: }\end{array}$ & 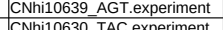 & \begin{tabular}{|l|} 
Negative control \\
Neqative control
\end{tabular} & NA & & 12|NA & Homo sapiens & $\begin{array}{l}\text { FANTOM6 project } \\
\text { FANTOM6 proiect }\end{array}$ & $\begin{array}{l}\text { PRJDB79993 } \\
\text { PRJPR7939 }\end{array}$ & \begin{tabular}{|r|r|}
11157330 \\
11568347
\end{tabular} & & 17CAGE & ASO_NCAA & $\begin{array}{l}\mathrm{HDF} \text { (neonat) } \\
\mathrm{HDF} \text { (neopatat }\end{array}$ \\
\hline 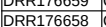 & 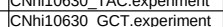 & \begin{tabular}{|l|l|l|l|l|l|l|} 
\\
Targeted control
\end{tabular} & NA & & 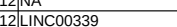 & 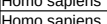 & poroject & 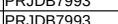 & $\begin{array}{l}\mid 456834 t \\
14061748\end{array}$ & & $\begin{array}{ll}7 \mathrm{ACAE} \\
7 \mathrm{CAGE}\end{array}$ & ASO & HDF (neonat \\
\hline R176 & CNhi110630 GCG Ge. & Tagrated & NA & & 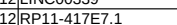 & Homo sapiens & project & PRJD & 12365942 & & 列CALE & ASO & $\begin{array}{l}\text { HDLF (neon } \\
\text { HDF neon }\end{array}$ \\
\hline R176 & CNhil10630_CAC.experiment & Targeted & NA & & 12 C11orf95 & Homo sapiens & 16 project & & 13926089 & & 17CAGE & ASO_G0188070_AD_05 & HDF (neor \\
\hline 5 & CNhil10630_ATG.experiment & Targeted & NA & & 12 ERVK13-1 & Homo sapiens & project & & 15897264 & & 17CAGE & ASO_G0260565_AD_06 & HDF (neonal \\
\hline DRR17 & 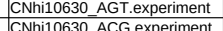 & $\begin{array}{l}\text { Targeted } \\
\text { Targeted }\end{array}$ & NA & & $\begin{array}{l}12 \mathrm{RP1} 1 \\
12 \mathrm{RPG}\end{array}$ & $\begin{array}{l}\text { Homo sapiens } \\
\text { Homo sapiens }\end{array}$ & project & & $\begin{array}{ll}11413198 \\
1401629 \\
\end{array}$ & & $\begin{array}{ll}17 C A G E \\
717 C A E\end{array}$ & \begin{tabular}{|l} 
ASO_G0223 \\
ASO G0270
\end{tabular} & $\begin{array}{l}\text { HDF (neonat } \\
\text { HDF (neonat }\end{array}$ \\
\hline RR176 & 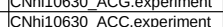 & $\begin{array}{l}\text { Talgelee } \\
\text { Targeted }\end{array}$ & INA & & $\begin{array}{l}\frac{112 R P 6}{12 R P 1} \\
12 R 1\end{array}$ & $\begin{array}{l}\text { Homo sapentiens } \\
\text { Homo sapiens }\end{array}$ & $\begin{array}{l}\text { poroect } \\
\text { project }\end{array}$ & $\begin{array}{l}\text { PRJ } \\
\text { PRI }\end{array}$ & | & & $\begin{array}{ll}7 \text { CAEE } \\
77 C A G E\end{array}$ & & DDF (neona \\
\hline RR176587 & CNhi10005 GCT E. experiment & Targeted & $\mathrm{NA}$ & & & Homo sapiens & project & & 535389 & & 17CAGE & 701 & \\
\hline RR17 & CNhi10005 GCG.experiment & Targeted & NA & & 10 & Homo sapiens & FANTOM6 project & PRJDB7993 & 10382541 & & 17CAGE & ASO $=0241990.07$ & HDF (neona \\
\hline DRR176585 & CNhi10605_CAC.experiment & Targeted & NA & & 10 LINC00674 & Homo sapiens & FANTOM6 project & PRJDB7993 & 13123013 & & 17CAGE & ASO_G0237854_05 & HDF (neona \\
\hline DRR17 & CNhi106 & $\begin{array}{l}\begin{array}{l}\text { Experime } \\
\text { control }\end{array} \\
\text {. }\end{array}$ & NA & & 10|MALA & & $\mathrm{ct}$ & 3 & 394 & & 17 CAGE & & HDF $(n$ \\
\hline URRI & CNhi10605_A & Targeted & $N A$ & & S1 & Homo sapiens & $F A$ & & 16349504 & & 17CAGE & ASO_C0247572_09 & HUTF (I \\
\hline $\begin{array}{l}\text { RR176582 } \\
\text { DRP176501 }\end{array}$ & CNhili0605_ACG.experiment & Targeted & NA & & 111LII & Homo sapiens & project & & 634 & & & ASO_G0243701_01 & HDF (neo \\
\hline $\begin{array}{l}\text { DRR176581 } \\
\text { DRR176580 }\end{array}$ & 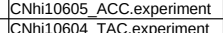 & $\begin{array}{l}\text { Targeted } \\
\text { Targeted }\end{array}$ & NA & & $\begin{array}{lll}11 \mid \mathrm{TP5} 5 \\
104 \mathrm{UNC}\end{array}$ & $\begin{array}{l}\text { Homo sapiens } \\
\text { Homo sapiens }\end{array}$ & project & & $\begin{array}{l}16141896 \\
12703583\end{array}$ & & 17CAGE & $\begin{array}{l}\text { ASO_G0182165_AD_10 } \\
\text { ASO G0238266 }\end{array}$ & $\begin{array}{l}\text { HDF (neonat } \\
\text { HF (neonat }\end{array}$ \\
\hline $\begin{array}{l}\text { ORRR16580 } \\
\text { DRR176515 }\end{array}$ & 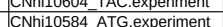 & $\begin{array}{l}\mid \text { Iargeted } \\
\text { Targeted } \\
\end{array}$ & $\frac{N A}{N A}$ & & & $\begin{array}{l}\text { Homo Sapiens } \\
\text { Hom sains }\end{array}$ & 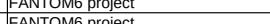 & & 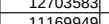 & & ICAEE & 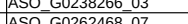 & HDF (neonal \\
\hline & 884 AGT. experiment & $\begin{array}{l}\text { Talgeted } \\
\text { Targeted } \\
\end{array}$ & NA & & $\begin{array}{l}9 / R P 11 \\
9 / 211\end{array}$ & Homo sapiens & project & & \begin{tabular}{|l|}
1109549 \\
1306899
\end{tabular} & & 77 CAGE & ASO G0262468 04 & $\begin{array}{l}\text { Hivi (neona } \\
\text { HDF (neona }\end{array}$ \\
\hline 烈 & CNhi10584_ACG.e & Targ & NA & & 9[L & Homo sapiens & project & PRJD & 11886738 & & 77CAGE & ASO_G0263753_ & HDF (neo \\
\hline DRR17 & CNhi10584_ACC.experiment & Targ & NA & & $9 R^{2}$ & Homo sapiens & project & & 19111059 & & 17CAGE & SO G & HDF (neor \\
\hline DRR176 & CNhnil0583_TAC.experiment & $\begin{array}{l}\text { Targeted } \\
\text { Tarotend }\end{array}$ & NA & & 9RP11-333E1.1 & Homo sapiens & project & $\begin{array}{l}P \text { PRJ } \\
\text { PBI }\end{array}$ & \begin{tabular}{|l|l|}
11345714 \\
10793275
\end{tabular} & & 17CAGE & SO_G0261879_05 & HDF (neona \\
\hline & CNh110583-G & & NA & & & Homo sapiens & & & & & 和 CAGE & $\frac{02}{23}$ & HDF (neonat \\
\hline & iment & $\begin{array}{l}\text { Talgeted } \\
\text { Targeted } \\
\end{array}$ & $\mathrm{NA}$ & & $9 \mathrm{RP}$ & Homo & 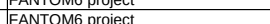 & & & & & $=002$ & HDF (neonal \\
\hline & ment & 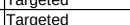 & $\frac{N A}{N A}$ & & & lomo & & & & & $\frac{C A}{C A}$ & & (neona \\
\hline+6 & CNhi10543-A & Targeted & & & 5.CATG000000079799.1 & Homo sapiens & project & PRJI & 11721046 & & & (08586 03 & HDF (neo \\
\hline DRR. & CNhi10543_ACC.experiment & Negative co & NA & & $5 \mid$ & Homo sapiens & project & & 12082179 & & CAGE & NC_A & HDF (neona \\
\hline & iment & Refer & NA & & $0 \mid \mathrm{NA}$ & Homo & roject & & 117539266] & & FAGE & & HDF (neonat) \\
\hline & CN & Nege & NA & & & $\begin{array}{l}\text { Homo } \\
\text { Homo }\end{array}$ & & & & & & & $\mathrm{HD}$ \\
\hline & & & NA & & & & & & & & & & \\
\hline & ment & & $N A$ & & & Hom & & & & & GE & & $\frac{\mathrm{HD}}{\mathrm{HD}}$ \\
\hline & CNhi105 & & $\mathrm{N} / \mathrm{s}$ & & & Hor & ct & & & & EE & 07 & $\mathrm{HD}$ \\
\hline & eriment & Targe & NA & & & & & & 10 & & SE & 5003 & ATL \\
\hline & ment & Iargeted & IA & & & s & & & & & & & $\mathrm{HD}$ \\
\hline & CN & Trarae & NAA & & & & & & & & & & \\
\hline & & & $\frac{F A}{N A}$ & & & & & & & & & & \\
\hline & CNhi10533 & Negeg 2 & $\mathrm{NA}$ & & & Hom & & & & & $5 E$ & & \\
\hline & $\mathrm{CNh}$ & & $\mathrm{NA}$ & & & & $F F$ & & & & & & \\
\hline & eriment & Targated & NA & & & & & & & & $\mathrm{E}$ & & $\mathrm{HD}$ \\
\hline & ent & Targ & IA & & (CD99P1 & & & & 䛲64 & & & & \\
\hline & & & & & & & & & & & & & \\
\hline 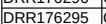 & CNhi10524 & $\begin{array}{ll}\text { Inege } \\
\text { Targ }\end{array}$ & & & 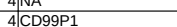 & Hom & FAn & & 17651165 & & CAGE & & \\
\hline
\end{tabular}


Table_S3 Meatadata for single cell analysis and FANTOM6 kmer research

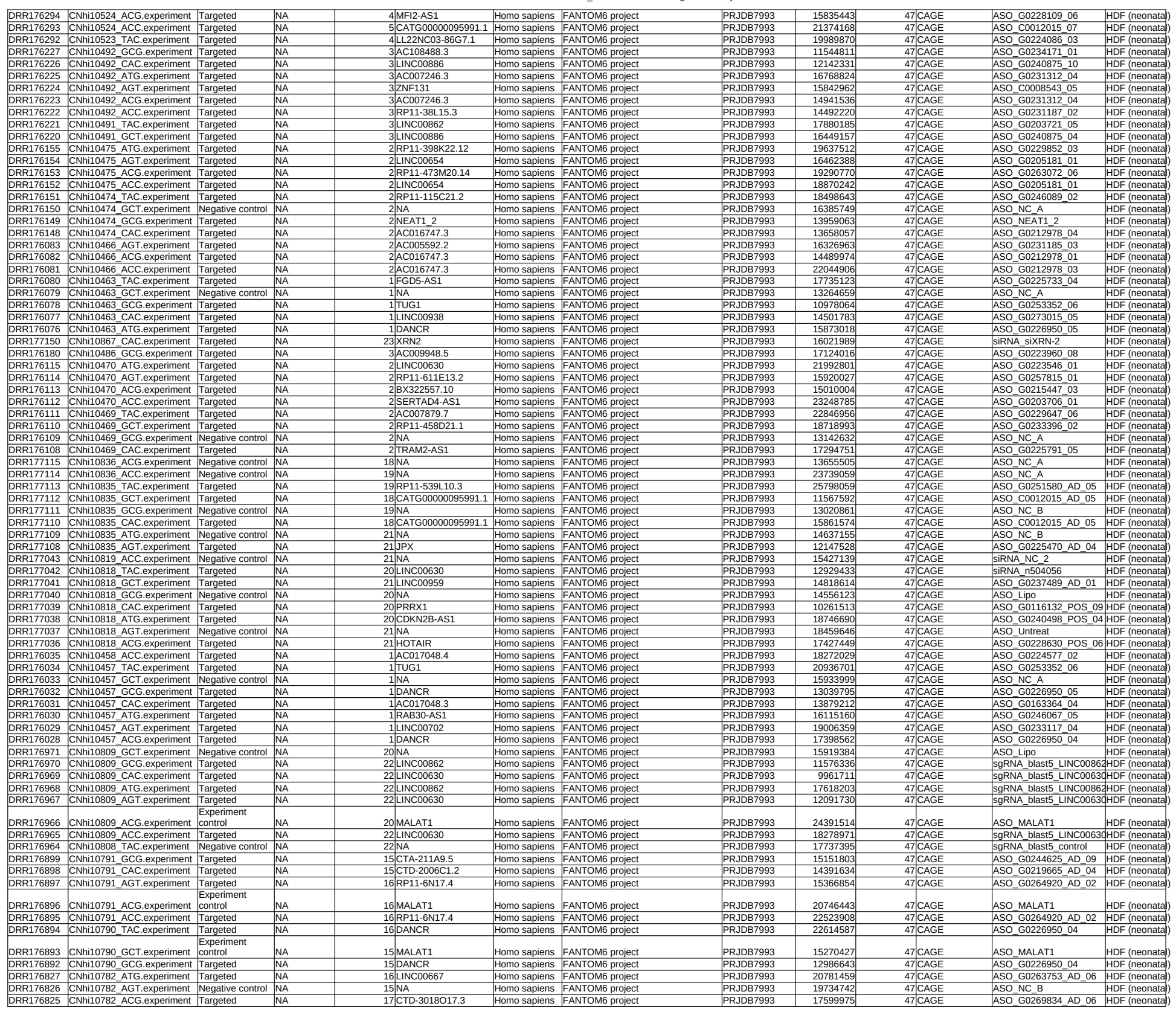


Table_S3 Meatadata for single cell analysis and FANTOM6 kmer research

\begin{tabular}{|c|c|c|c|c|c|c|c|c|c|c|c|}
\hline $\begin{array}{l}\text { DRR116824 } \\
\text { DRR176823 }\end{array}$ & 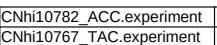 & $\begin{array}{l}\text { ve control } \\
\text { ve control }\end{array}$ & $\frac{N A}{N A}$ & $\begin{array}{l}16 \mid \mathrm{NA} \\
13 \mathrm{NA}\end{array}$ & \begin{tabular}{|l|} 
Homo sapiens \\
Homo sapiens
\end{tabular} & $\begin{array}{l}\text { FANTOM6 project } \\
\text { FANTOM6 project }\end{array}$ & \begin{tabular}{|l} 
PRJDB79933 \\
PRJDB79933
\end{tabular} & \begin{tabular}{|l|}
23277079 \\
21972404 \\
\end{tabular} & $\begin{array}{l}47 \text { CAGE } \\
47 \text { CAGE }\end{array}$ & \begin{tabular}{|l} 
ASO_Lipo \\
ASO_Lipo
\end{tabular} & $\begin{array}{l}\text { HDF (neonatat) } \\
\text { HDF (neonatat) }\end{array}$ \\
\hline $\begin{array}{ll}\text { DRR176822 } \\
R 17822\end{array}$ & $\begin{array}{ll}\text { CNNhi10767GCT.experiment } \\
\end{array}$ & 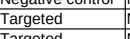 & $\frac{N A}{N A}$ & $\begin{array}{ll}13 \mathrm{MEG} 3 \\
13 \mathrm{MEF} 2\end{array}$ & $\begin{array}{l}\text { Homo sapiens } \\
\text { Homo sains } \\
\end{array}$ & 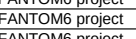 & 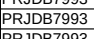 & $\begin{array}{l}11757678 \\
1175678 \\
\end{array}$ & 47TCAGE & ASO_60214548_AD_06 & HDF (neonatat) \\
\hline & Nhi10767_GCG.experiment & & NA & $\begin{array}{l}14 \text { MEG3 } \\
\end{array}$ & Homo sapiens & FANTOM6 project & $\begin{array}{l}\text { PRJDB79933 } \\
\text { DPIDP7093 }\end{array}$ & 10872425 & 477CAGE & ASO_G0214548_AD_06 & HDF (neonatat) \\
\hline 55 & $\begin{array}{l}\text { CNNin107767CAC.experiment } \\
\text { CNNiin0759 ACG experiment }\end{array}$ & $\begin{array}{l}\text { Targeted } \\
\text { Torageted }\end{array}$ & $\frac{N A}{N A}$ & $\begin{array}{l}\text { 17/RP11-611E13.2 } \\
\text { 14RP1-173B14.5 }\end{array}$ & \begin{tabular}{|l} 
Homo sapiens \\
Homo saaiens
\end{tabular} & $\begin{array}{l}\text { FANTOM6 project } \\
\text { FANTOM6 project }\end{array}$ & $\begin{array}{l}\text { PRJJD79993 } \\
\text { PRJDB7993 }\end{array}$ & \begin{tabular}{|l}
16922148 \\
19769317 \\
\end{tabular} & $\begin{array}{l}\text { 47CAGE } \\
47 \text { CAGE }\end{array}$ & \begin{tabular}{|l|} 
ASO_G0257815_AD_03 \\
ASO G0261105 AD 10
\end{tabular} & $\begin{array}{l}\text { HDF (neonatat) } \\
\text { HDF (neonatat) }\end{array}$ \\
\hline & 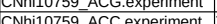 & largeled & NA & (1) & 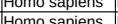 & 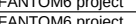 & 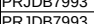 & 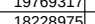 & $\begin{array}{l}\text { 47CAGE } \\
7 \text { TCAGE }\end{array}$ & 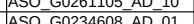 & HDF (neonatat) \\
\hline & $\begin{array}{l}\text { ACC.experiment } \\
\text { T TAC.experiment }\end{array}$ & Tarageted & NA & 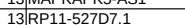 & Homo sapiens & $\begin{array}{l}\text { FANTOM6 project } \\
\text { FANTOM6 project }\end{array}$ & PRJBD7933 & 22627200 & 47CACE & 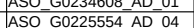 & $\begin{array}{l}\text { HDF (neenatal) } \\
\text { HDF (neoonatat) }\end{array}$ \\
\hline DRR176752 & CNhii0758_GCT.experiment & $\begin{array}{l}\text { Targected } \\
\text { Targeted }\end{array}$ & NA & 13RP11-139H15.1 & Homo sapiens & FANTOM6 project & PRJDB7993 & 16023407 & 477CAGE & ASO_G0225973_AD_01 & $\begin{array}{l}\text { HDLF (neonate } \\
\text { HDF (neonata }\end{array}$ \\
\hline DRR176751 & CNhii10758_GCG.experiment & $\begin{array}{l}\text { Targeted } \\
\end{array}$ & NA & 14 LINC00517 & Homo sapiens & FANTOM6 project & PRJDB7993 & $\begin{array}{l}13517430 \\
\end{array}$ & & & \\
\hline DRR176750 & CNhi10758 CAC.experiment & 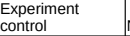 & NA & 14 MALAT1 & \begin{tabular}{|l|} 
Homo sapiens \\
\end{tabular} & FANTOM6 project & PRJDB7993 & 14146389 & 47 CAGE & ASO MALAT1 & HDF (neonat \\
\hline & CNhi10758_ATG.experiment & & NA & $13 \mathrm{NA}$ & & (6 project & & $\begin{array}{l}8205374 \\
\end{array}$ & 47CAGE & ASO_Untreat & HDF (neon \\
\hline $\begin{array}{ll}\frac{R}{R R 176748} \\
\text { R1776683 }\end{array}$ & 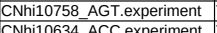 & $\begin{array}{l}\text { Targeted } \\
\text { Taranted }\end{array}$ & $\frac{N A}{N A}$ & $\begin{array}{l}13 \text { MAPKAPK5-AS1 } \\
12 \text { IER/13-1 }\end{array}$ & \begin{tabular}{|l} 
Homo sapiens \\
Homo saniens
\end{tabular} & $\begin{array}{l}\text { FANTOM6 project } \\
\text { FANTM6 project }\end{array}$ & $\begin{array}{l}\text { PRJDB79933 } \\
\text { PRID7993 }\end{array}$ & $\begin{array}{l}18799556 \\
1650405 \\
\end{array}$ & $\begin{array}{ll}47 C A G E \\
47 C A G E\end{array}$ & ASO_G0234608_AD_01 & $\begin{array}{l}\mathrm{HDF} \text { (neonat } \\
\mathrm{HDF} \text { (neonat }\end{array}$ \\
\hline$\frac{3}{2}$ & 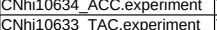 & $\begin{array}{l}\text { Targeted } \\
\text { TTargeted }\end{array}$ & $\frac{N A}{N A}$ & & \begin{tabular}{|l|} 
Homo sapiens \\
Homo sapiens
\end{tabular} & $\begin{array}{l}\text { FANTOM6 project } \\
\text { FANTOM6 prject }\end{array}$ & $\begin{array}{l}\text { PRJUD/993 } \\
\text { PRDB7993 }\end{array}$ & \begin{tabular}{|l|}
167500405 \\
13894549 \\
\end{tabular} & $\begin{array}{l}\text { 477CAGE } \\
47 \text { CAGE }\end{array}$ & $\frac{\mid \mathrm{ASO} C \mathrm{C}}{\mathrm{ASO} \mathrm{C}}$ & $\frac{\mathrm{HDF} \text { (neona }}{\mathrm{HDF} \text { (neona }}$ \\
\hline & & & NA & & & & PRJPB T993 & 14931786 & 47CCAGE & & $\begin{array}{l}\text { HDF (neonat } \\
\text { HDF (neonat }\end{array}$ \\
\hline DRR176680 & CNhin 10633 GCG Gevperiment & Tarageted & $\frac{N A}{N A}$ & 12 LINC00339 & $\begin{array}{l}\text { Homo sapiens } \\
\text { Homo sen }\end{array}$ & $\begin{array}{l}\text { FANTOMG project } \\
\text { A }\end{array}$ & PRJDB7993 & 13046523 & 47. CAGE & ASO G0218510 AD 06 & $\begin{array}{l}\text { HDF (neonat } \\
\mathrm{HDF} \text { (neonat }\end{array}$ \\
\hline DRR176679 & CNhii10633_CAC.experiment & Targeted & NA & $12 \mathrm{MEG} 3$ & Homo sapiens & FANTOM6 project & PRJDB7993 & 15656972 & 47 CAGE & ASO_G0214548_AD_06 & HDF (neonat \\
\hline $\begin{array}{ll}176678 \\
\text { R176677 }\end{array}$ & 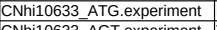 & Negative control & NA & 12 NA & Homo sapiens & FANTOM6 project & \begin{tabular}{|l} 
PRJDB7993 \\
DDID7092
\end{tabular} & 13781324 & 47CAGE & ASO_NC_A & \\
\hline $\begin{array}{l}\text { DRR1166677 } \\
\text { DRR176676 }\end{array}$ & 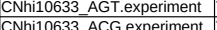 & $\begin{array}{l}\text { Targeted } \\
\text { TTargeted }\end{array}$ & NA & $\begin{array}{l}\text { 12RAB30-AS1 } \\
\text { 12DANCR }\end{array}$ & Homo sapiens & FANTOM6 project & PRJDB79933 & \begin{tabular}{|l|}
13191782 \\
14220102 \\
\end{tabular} & $\begin{array}{l}\text { 47CAGE } \\
\text { A7CAGE }\end{array}$ & \begin{tabular}{|l|} 
ASO_G0206067_AD_09 09 \\
ASO G026950 AD 07
\end{tabular} & $\begin{array}{l}\text { HDF (neonat) } \\
\text { HDE (neonat }\end{array}$ \\
\hline $\begin{array}{l}\text { DRR16661 } \\
\text { DRR176611 }\end{array}$ & 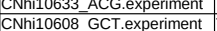 & $\begin{array}{l}\text { Tlargelea } \\
\text { Targeted }\end{array}$ & NA & $\begin{array}{l}\text { 12LDANCR } \\
10 \text { LINC00959 }\end{array}$ & & $\begin{array}{l}\text { FANTOM6 p poject } \\
\text { FANTOM6 proect }\end{array}$ & $\begin{array}{l}\text { PRJDL/9953 } \\
\text { PRJDB7993 }\end{array}$ & \begin{tabular}{|}
14220102 \\
13215357 \\
\end{tabular} & $\begin{array}{l}\text { 47CAGE } \\
47 \text { CAGE }\end{array}$ & & 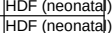 \\
\hline & CNhi10608 GCG Gxperiment & & $\frac{N A}{N A}$ & 10 107SAN16-AS1 & & & PRJPB7993 & 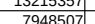 & (47CACE & ASO G02692930 & $\begin{array}{l}\text { HDF (neenata) } \\
\text { HDF (neoonatat) }\end{array}$ \\
\hline$\frac{069}{609}$ & CNhii10608 CAC.experiment & $\begin{array}{l}\text { Talageleu } \\
\text { Targeted }\end{array}$ & $\mathrm{NA}$ & 10 ZNF730 & Homo sapiens & FANTOMG project & $\frac{1}{\text { PRJDBR7993 }}$ & 14389504 & 477CAGE & ASO G02678860 03 & HDF (neoonat \\
\hline DRR176608 & CNni10608_ATG.experiment & Targeted & NA & 11 RAB30-AS1 & Homo sapiens & FANTOM6 project & PRJDB7993 & 9486804 & 47 CAGE & ASO_G0246067_01 & HDF (neonat \\
\hline & CNhi10608_AGT.experiment & $\begin{array}{l}\text { Targeted } \\
\text { Trantod }\end{array}$ & NA & 11 LINC00883 & Homo sapiens & FANTOM6 project & $\begin{array}{l}\text { PRJDB79933 } \\
\text { DPID7093 }\end{array}$ & $\begin{array}{l}11275756 \\
105622\end{array}$ & 477CAGE & ASO_G0243701_AD_09 & HDF (neonat \\
\hline DRR176606 & CNhi10608_ACG.experiment & $\begin{array}{l}\text { Targeted } \\
\text { Expeniment }\end{array}$ & NA & 10RP11-11011.12 & Homo sapiens & FANTOM6 project & PRJDB7993 & & 477CAGE & & HDF (neonal \\
\hline DRR176605 & CNhi10608_ & 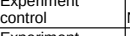 & NA & 10 MALAT1 & Homo sapiens & FANTOM6 project & PRJDB7993 & 10630460 & 47) CAGE & ASO_MALA & HDF (neon \\
\hline DRR176604 & experiment & $\begin{array}{l}\text { Experime } \\
\text { control }\end{array}$ & NA & 10|MALAT1 & Homo sapiens & FANTOM6 project & PRJDB7993 & 12814570 & 47) CAGE & ASO_MALAT1 & HDF (neon \\
\hline DRR176539 & CNhi10587_CAC.experiment & $\begin{array}{l}\text { Targeted } \\
\text { Fygetions }\end{array}$ & NA & $\begin{array}{l}\text { 9 CTC-559E9.6 } \\
\end{array}$ & Homo sapiens & FANTOM6 project & PRJDB7993 & $\begin{array}{l}11728992 \\
\end{array}$ & 477CAGE & ASO_60267419_02 & HDF (neon \\
\hline & Nhii105e & $\begin{array}{l}\text { Experime } \\
\text { control }\end{array}$ & NA & 9 MALAT1 & Homo & ANTOM6 project & 3 & 12599727 & 47) CAGE & & \\
\hline 77 & CNhi10587_AGT.expe & Targeted & NA & $9 A C 00900$ & Homo sapiens & FANTOM6 project & PRJDB7993 & 8446 & 477CAGE & $\mathrm{ASO}_{-}$ & HDF (ne \\
\hline DRR176536 & experiment & $\begin{array}{l}\text { Experiment } \\
\text { control }\end{array}$ & NA & 9 MALAT1 & Homo sapiens & FANTOM6 project & & 1244674 & 47 CAGE & & \\
\hline & CNhi10s & Targeted & NA & 9 CTC-559E9.6 & Homo sapiens & rject & PRJDB7993 & $\frac{1144}{1739 !}$ & $\begin{array}{l}4 \pi \text { CALE } \\
477 \text { CAGE }\end{array}$ & & HDF (ned \\
\hline DRR176534 & CNhi10586_TAC.experiment & Targeted & NA & 9|CTD-2319112.4 & Homo sapiens & FANTOM6 project & PRJDB7993 & 12397936 & 47 CAGE & ASO_G0267248_02 & HDF (neona \\
\hline & CNhi10586_GCT.expe & Targeted & NA & 9.CTD-2162K18.5 & Homo sapiens & FANTOM6 project & $\begin{array}{l}\text { PRJDB79933 } \\
\text { DPID7093 }\end{array}$ & 13818504 & 477CAGE & ASO_G0267254_07 & HDF (neone \\
\hline $\begin{array}{l}\text { DRR16532 } \\
\text { DRR176467 }\end{array}$ & 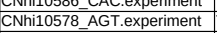 & $\begin{array}{l}\text { Targelea } \\
\text { Targeted }\end{array}$ & NA & 6PITPNA-AS1 & $\begin{array}{l}\text { |Homo sapiens } \\
\text { Homo sapiens } \\
\end{array}$ & $\begin{array}{l}\text { IAANTOM 6roject } \\
\text { FANTOM6 project }\end{array}$ & $\begin{array}{l}\text { PRJJB79993 } \\
\text { PRJDB7993 }\end{array}$ & \begin{tabular}{|l}
11299949 \\
15144036 \\
\end{tabular} & $\begin{array}{l}\text { 477CAGE } \\
47 \text { CAGE }\end{array}$ & 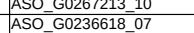 & $\begin{array}{l}\text { HDF (neonat } \\
\text { HDF (neonat }\end{array}$ \\
\hline DRR176466 & CNhi10578 ACG.experiment & $\begin{array}{l}\text { Experiment } \\
\text { control }\end{array}$ & NA & 6 MALAT1 & Homo sapiens & 6 project & & 213 & 47 CAGE & & HDF (neor \\
\hline & experiment & Targeted & NA & 6 LINC00263 & Homo sapiens & project & PRJDB7993 & 140 & 47 CAGE & ASO_60235823_03 & HDF (neo \\
\hline & Ac.exper & Targeted & NA & 6/RP11-357C3.3 & Homo sapiens & 6 project & PRJDB7993 & $\begin{array}{l}16395136 \\
\end{array}$ & 47CAGE & ASO_G0235437_09 & HDF (neon, \\
\hline 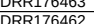 & 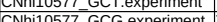 & Targeted & $\frac{N A}{N A}$ & (1) & H Homo sapens & $\begin{array}{l}\text { FANTOM6 project } \\
\text { FANTOMG6roiect }\end{array}$ & $\begin{array}{l}\text { PRJDB79993 } \\
\text { PRDBR7993 }\end{array}$ & $\begin{array}{l}13356982 \\
15790272 \\
\end{array}$ & $\begin{array}{ll}47 \text { CAGE } \\
47 \text { CAGGE }\end{array}$ & 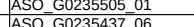 & HDF (neonat \\
\hline 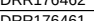 & 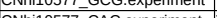 & Trageled & $N A$ & 6PP11.257c3 & tyomo sapions & thorect & (1) & 152814 & & 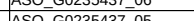 & HDF (neonate \\
\hline 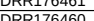 & 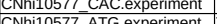 & Trageted & NA & 6 & $\begin{array}{l}\text { H Homo saplens } \\
\text { tumo }\end{array}$ & forpiont & DP107093 & 1308192 & T7CAGE & A & IDFF (neonatic \\
\hline & AIGGexperiment & $\begin{array}{l}\text { Talageleu } \\
\text { Targeted }\end{array}$ & $\mathrm{NA}$ & 5FLVCR1-AS1 & $\begin{array}{l}\text { Homo sapiens } \\
\text { Homo sapiens }\end{array}$ & $\frac{10 \text { project }}{16}$ & $\frac{1}{\text { PRJDBR7993 }}$ & $\frac{35}{35}$ & 477CAGE & ASO G0198468 & $\begin{array}{l}\text { HDF (neonat } \\
\text { HDF (neonat }\end{array}$ \\
\hline DRRI & CNhii10537_ACC.experiment & Targeted & NA & 6 CTC-338M12.4 & Homo sapiens & project & PRJDB7993 & 13596462 & 47 CAGE & ASO_G0233937_05 & HDF (neona \\
\hline & TAC.experiment & Targeted & NA & 6 (MAGI2-AS3 & Homo sapiens & project & |PRJDB7993 & 14464102 & 477CAGE & ASO_G0234456__4 & HDF (neonata) \\
\hline & CNhi10536_GCT.experiment & Targented & NA & 5FLVCR1-AS1 & Homo sapiens & 16 project & & 17180713 & 47TCAGE & ASO_60198468_01 & HDF (neonatat) \\
\hline & CNhiilo & Targeted & & & & & & & & & \\
\hline & Fexperiment & Negative control & NA & 5 NA & Homo sapiens & project & |PRJDB7993 & $\begin{array}{l}11749411 \\
\end{array}$ & 47 CAGE & ASO_NC_A & HDF (neona \\
\hline & S & largelea & $\frac{N A}{N A}$ & (1) & 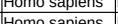 & project & 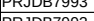 & 11365544 & $\begin{array}{l}\text { 47CAGE } \\
7 \text { TCAGE }\end{array}$ & 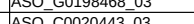 & HDF (neon, \\
\hline & The experinemt & Targeter & 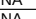 & ACATCT1D & thome sapions & (a) & (2) & 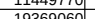 & ATCACE & $A C=C 0206230$ & HDF (neon \\
\hline & 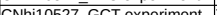 & Trageted & NA & & 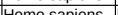 & t & DPIDT70093 & 17776122 & A7CAGE & ASO C Co000830 02 & HDF (neon \\
\hline DRR176321 & the & TTarated & NA & $\frac{1}{4 R P P 11-395 B 7.4}$ & $\begin{array}{l}\text { Homosapiens } \\
\text { Homo sains }\end{array}$ & th & PRJDB7993 & 13714212 & $\begin{array}{l}47 \text { CAGE } \\
\text { 47CAGE }\end{array}$ & A ASO G022200504 & $\begin{array}{l}\text { HDF (neen } \\
\text { HDF (neoon }\end{array}$ \\
\hline DRR: & CNhi10527_CAC.expe & Targeted & NA & 3A18.4 & Homo sapiens & 6 project & PRJDB7993 & 18580280 & 477CAGE & ASO $=$ & HDF (neona \\
\hline & S.experiment & Targeted & NA & 00000020700.1 & Homo sapiens & ect & PRJD & & 47 CAGE & 0984_09 & HDF (neona \\
\hline & T.experiment & Targeted & NA & 4|PCAT & Homo sapiens & project & PRJDB7993 & 18372099 & $47 \mathrm{C} / \mathrm{C} / \mathrm{C}$ & ASO_G0228288_01 & HDF (neon, \\
\hline & CG.experiment & Negative control & INA & & Homo sapiens & FANT & PRJDB7993 & 18247767 & 477CAGE & ASO_NC_A & \\
\hline & & & & & Homo sapiens & FANT & PRJDE & & 47 CAGE & ASO_C0000830_08 & \\
\hline & T.experiment & Targented & NA & 3LINC00886 & Homo sapiens & 6 project & PRJDB7993 & 12730601 & 47/CAGE & ASO_G02407075_04 & \\
\hline & 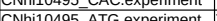 & Tarageted & $\frac{N A}{N A}$ & 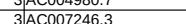 & $\begin{array}{l}\text { Homo sapiens } \\
\text { Homo sapiens }\end{array}$ & $\begin{array}{l}6 \text { project } \\
6 \text { 6roject }\end{array}$ & PRJDP793 & 100763090 & $\begin{array}{l}\text { 47CACE } \\
47 \mathrm{CACE}\end{array}$ & 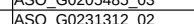 & HDF (neona \\
\hline$\frac{D R F}{D R F}$ & 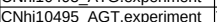 & ( & NA & & Ho & ct & PRJDB7993 & $\begin{array}{ll}11403597] \\
\end{array}$ & $47 \mid \mathrm{C}$ & (1) & \\
\hline & 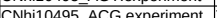 & Trancered & NAA & 3 CTD-2587H24.5 & Homo saniens & intopect & PRIDB7993 & 10939 & $A 7 C A G$ & $A S O G C$ & 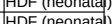 \\
\hline DRR & SACGexperiment & $\begin{array}{l}\text { Targeted } \\
\text { Targed }\end{array}$ & NA & 3LINCO0856 & $\begin{array}{l}\text { Homosaptiens } \\
\text { Homo saien }\end{array}$ & $\frac{1}{\text { project }}$ & $\frac{1}{\text { PRJD }}$ & 12788498 & 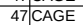 & D417-02 & $\begin{array}{l}\text { HDF (neonat } \\
\text { HDF (neonat }\end{array}$ \\
\hline $\begin{array}{l}\text { DRR176245 } \\
\text { DPD17624 }\end{array}$ & CNhi10494_TAC.experiment & Targeted & NA & 980.7 & Homo sapiens & 5 project & PRJDB7993 & 13771619 & 477CAGE & ASO_G & HDF (neonate \\
\hline & experiment & & NA & 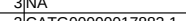 & & 政A & & 1244 & & ASONCNCA & $\mathrm{HDF}$ (neonat \\
\hline 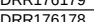 & 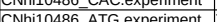 & $\begin{array}{l}\text { Jargeted } \\
\text { Taroted }\end{array}$ & 淔A & 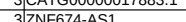 & thomo sapenins & FANTOME proetct & PRID7993 & 1432010 & 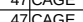 & ASO G020834011 & HDF (neona \\
\hline DRR176177 & CNhi10486 AGT experiment & Targated & NA & FBXL19-AS1 & Homo saaiens & FAI & 93 & 15900573 & 477CAGE & ASO G0260852 02 & HDF (neonat: \\
\hline & $\bar{A}$ ACG experiment & Neqative control & NA & & Homo sapiens & $\frac{F A}{F A}$ & PRJDB7993 & 11850730 & 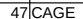 & & HEF (neonat \\
\hline DF & experiment & Targeted & NA & 3 ILINC & Homo sapiens & $\overrightarrow{F A}$ & PRJD & 170 & $47]$ & 01 & $\begin{array}{l}\text { HDF (neor } \\
\text { HDF neon }\end{array}$ \\
\hline & experiment & Targeted & NA & 12.1 & Homo sapiens & FAI & & & 477CAGE & & HDF \\
\hline & 7_GCT.experiment & Targeted & NA & 管699.1 & Homo sapiens & FANT & & 17651172 & 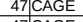 & ASO_C014559-06 & HDF (neonal \\
\hline 77 & CNhi10477_GCG.experiment & & $\frac{N A}{N A}$ & 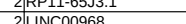 & Homo saniens & 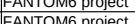 & (2) & 13482789 & CAGE & 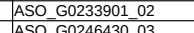 & HDF (neonat \\
\hline & & & & & & & & & & & \\
\hline
\end{tabular}


Table_S3 Meatadata for single cell analysis and FANTOM6 kmer research

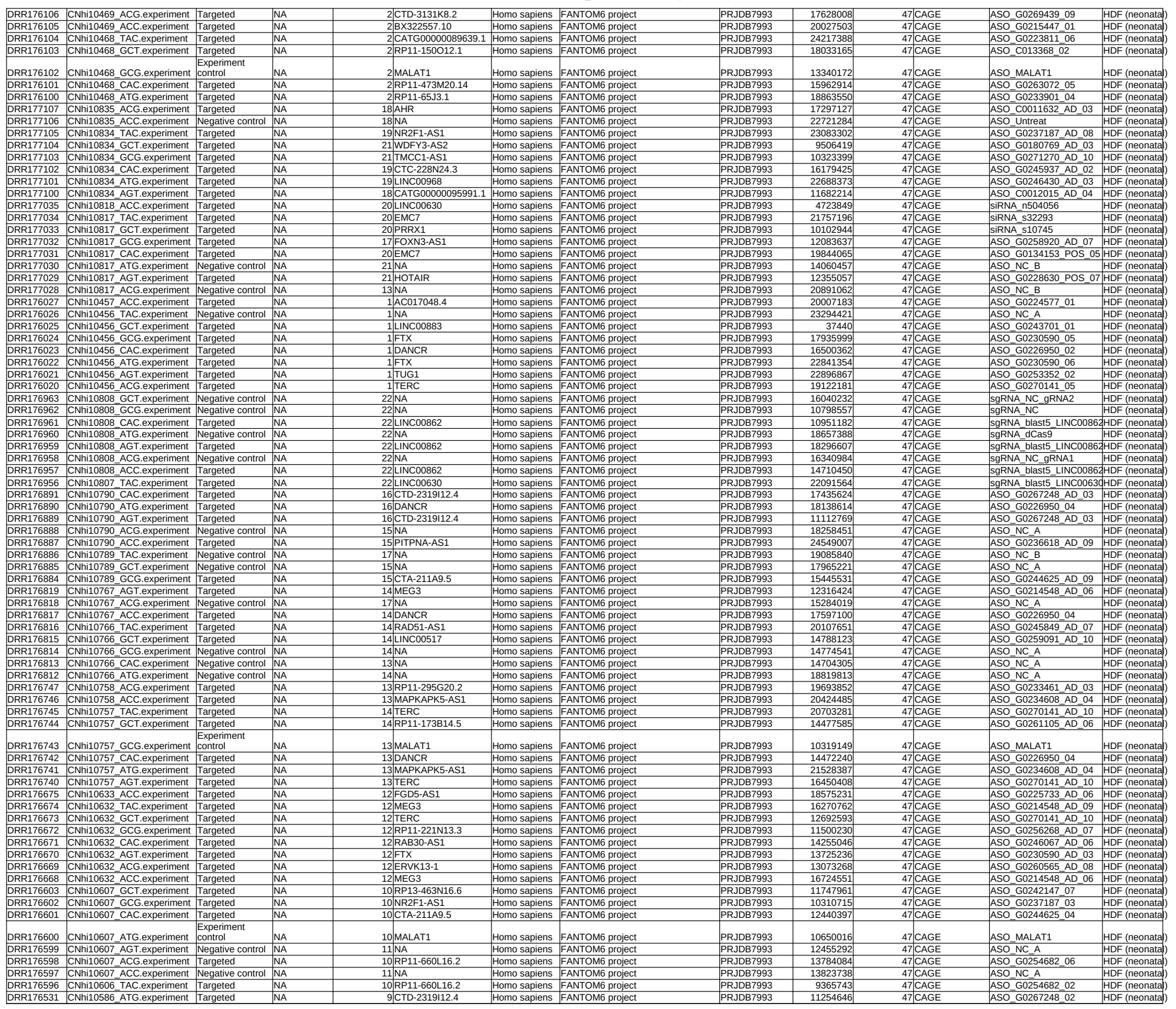

Page 11 
Table_S3 Meatadata for single cell analysis and FANTOM6 kmer research

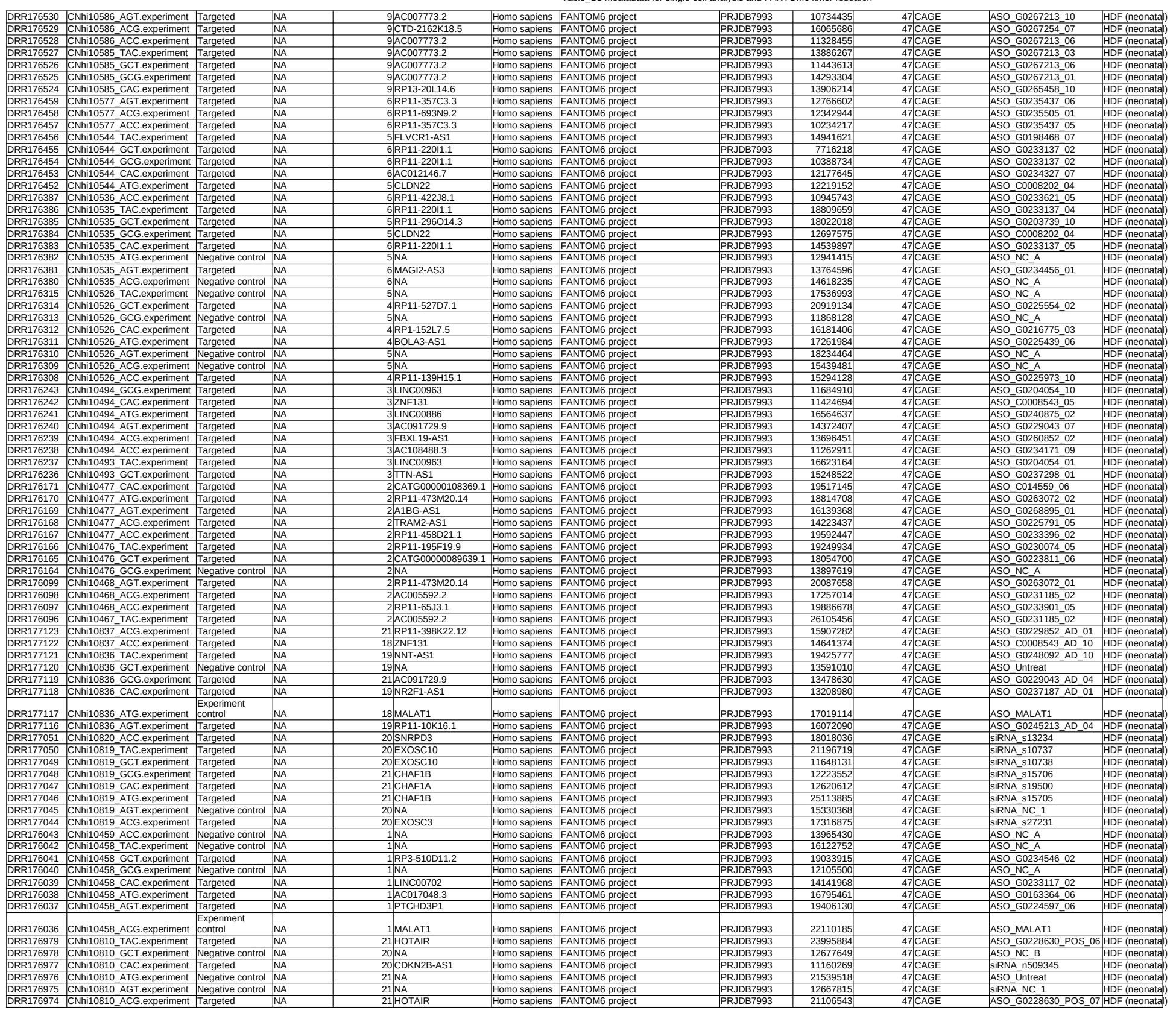


Table_S3 Meatadata for single cell analysis and FANTOM6 kmer research

\begin{tabular}{|c|c|c|c|c|c|c|c|c|c|c|c|}
\hline$\frac{D R R}{D R R}$ & 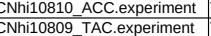 & $\begin{array}{l}\text { Targeted } \\
\text { TTraggeted }\end{array}$ & $\frac{N A}{N A}$ & $\begin{array}{l}21 \text { HOTAIR } \\
22 \text { LINC00862 }\end{array}$ & $\begin{array}{l}\text { Homo sapiens } \\
\text { Homo sapiens }\end{array}$ & $\begin{array}{l}\text { FANTOO6 project } \\
\text { FANTOM6 project }\end{array}$ & $\begin{array}{l}\text { PRJDD79993 } \\
\text { PRJDB7993 }\end{array}$ & $\begin{array}{l}19852143 \\
18022294 \\
\end{array}$ & 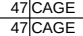 & $\frac{\text { SisNA ADAAYYu }}{\text { sgRNA blast5 LINC008E }}$ & $\frac{1 \mathrm{HDF} \text { (neonatal) }}{2 \mathrm{HDF} \text { (neounata) }}$ \\
\hline & & & NA & 2CATG00000089639.1 & Homo sapiens & FANTOM6 project & PRJDB7993 & 14925711 & 47 C7CAGE & & HDF (neonata), \\
\hline $\begin{array}{l}\text { DRR176094 } \\
\end{array}$ & CNhi10467_GCG.experiment & Negative control & NA & $2 \sqrt[N A]{ }$ & Homo sapiens & FANTOM6 project & PRJDB7993 & 13869436 & 47CAGE & ASO_NC_A & HDF (neone \\
\hline $\begin{array}{l}\text { DRR176093 } \\
\text { DRR176092 }\end{array}$ & CNNhi10467_CAC.experiment & $\begin{array}{l}\text { Targeted } \\
\text { TTareted }\end{array}$ & $\frac{\mathrm{NA}}{\mathrm{NA}}$ & $\begin{array}{l}\text { 2/RP11-398K22.12 } \\
\text { 2NEA1 } 1\end{array}$ & $\begin{array}{l}\text { Homo sapiens } \\
\text { Homo saniens }\end{array}$ & $\begin{array}{l}\text { FANTOM6 project } \\
\text { FANTOM6 project }\end{array}$ & $\begin{array}{l}\text { PRJJD79993 } \\
\text { PRJDB7993 }\end{array}$ & $\frac{16493655}{1732267}$ & $\begin{array}{l}47 \text { CAGE } \\
47 \text { CAGE }\end{array}$ & $\begin{array}{l}\text { ASO_G0229852_05 } \\
\text { ASO NEAT1 }\end{array}$ & $\begin{array}{l}\text { HDF (neonatat) } \\
\text { HFF (neoonata) }\end{array}$ \\
\hline $\begin{array}{l}\text { DRR16092 } \\
\text { DRR177099 }\end{array}$ & 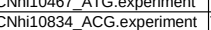 & $\begin{array}{l}\text { Larageted } \\
\text { Targeted }\end{array}$ & NA & $\begin{array}{l}\text { 2NDAII_1 } \\
\text { 19DANCR }\end{array}$ & $\begin{array}{l}\text { Homo saplens } \\
\text { Homo sapiens }\end{array}$ & $\begin{array}{l}\text { FANTOMG project } \\
\text { FANOM6 project }\end{array}$ & $\begin{array}{l}\text { PRJUB } \\
\text { PRJDB7993 }\end{array}$ & $\begin{array}{l}1 / 532464 \\
17620895 \\
\end{array}$ & $\begin{array}{l}4 \pi \text { CAGE } \\
477 \text { CAGE }\end{array}$ & $\begin{array}{l}\text { ASO__EATIII } \\
\text { ASO_G0226950_04 }\end{array}$ & 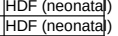 \\
\hline & CNhi10834 ACC.experiment & $\begin{array}{l}\text { Lalageteu } \\
\text { Targeted } \\
\end{array}$ & NA & $18 \mathrm{AHR}$ & $\begin{array}{l}\text { Homo sapiens } \\
\text { Homolus }\end{array}$ & FANTOM6 project & PRJDB7993 & 197405552 & 47 CAGE & $\mathrm{ASO}$ C0011632 AD 01 & $\begin{array}{l}\text { UDFF (neonatata) } \\
\text { UDF (neonata }\end{array}$ \\
\hline & CNhilo83__TAC.experiment & Negative control & NA & $19 \mathrm{NA}$ & Homo sapiens & FANTOM6 project & PRJDB7993 & 24301622 & 47 CAGE & ASO_Lipo & HDF (neonatala) \\
\hline 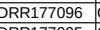 & CNhi10833_GCT.experiment & Targeted & NA & 19/MKLN1-AS2 & Homo sapiens & FANTOM6 project & PRJDB7993 & 12627254 & 477CAGE & ASO_G0236753_AD_01 & HDF (neone \\
\hline $\begin{array}{l}\text { DRR177095 } \\
\text { DRP177094 }\end{array}$ & CNhi100833_CAC.experiment & $\begin{array}{l}\text { Targeted } \\
\text { Trancted }\end{array}$ & NA & 19.DANCR & $\begin{array}{l}\text { Homo sapiens } \\
\text { Homp saiens. }\end{array}$ & FANTOM6 project & $\begin{array}{l}\text { PRJDB79933 } \\
\end{array}$ & 14096731 & 477CAGE & ASO_G0226950_04 & HDF (neon \\
\hline & 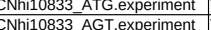 & $\begin{array}{l}\text { Targeted } \\
\text { Targeted }\end{array}$ & $\begin{array}{l}\text { NA } \\
\text { NA }\end{array}$ & $\begin{array}{l}\frac{19 \text { US }}{192111.14} \\
19 \text { RP11-539L10.3 }\end{array}$ & $\begin{array}{l}\text { Homo sapiens } \\
\text { Homo saiens }\end{array}$ & $\begin{array}{l}\text { FANTTM6 project } \\
\text { FANOM6 project }\end{array}$ & $\begin{array}{l}\text { PRJJD79993 } \\
\text { PRJDB7993 }\end{array}$ & 20193999 & $\begin{array}{l}47 \text { CAGE } \\
477 \text { CAGE }\end{array}$ & $\begin{array}{l}\text { ASO_G0232725_AD_10 } \\
\text { ASO G025150 AD } 01\end{array}$ & 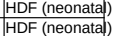 \\
\hline $\begin{array}{ll}\text { DRR177092 } \\
\text { DRR }\end{array}$ & CNnnivos33-AG_. & $\begin{array}{l}\text { Tlargeted } \\
\text { Targeted }\end{array}$ & NA & $\begin{array}{l}19 \mid \text { CATG000000095991.1 } \\
18 \text { CATG }\end{array}$ & $\begin{array}{l}\text { Homo saplens } \\
\text { Homo sapiens }\end{array}$ & $\begin{array}{l}\text { FANTIOM6 project } \\
\text { FANOM6 project }\end{array}$ & $\begin{array}{l}\text { PRJJER } \\
\text { PRJDB7993 }\end{array}$ & $\frac{13 / 29631}{20012008}$ & $\begin{array}{l}4 \pi \text { CAGE } \\
477 \text { CAGE }\end{array}$ & 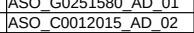 & $\begin{array}{l}\text { HDF (neen } \\
\text { HDF (neon }\end{array}$ \\
\hline & & & NA & 20 CDKN2B-AS1 & & & PRJDB79993 & 19349468 & 477CAGE & & HDF (neor \\
\hline DRR177026 & CNhilo816_TAC.experiment & Targeted & NA & 20PRRX1 & Homo sapiens & FANTOM6 project & PRJDB7993 & 16908584 & 47 CAGE & ASO_G0116132_POS_09 & $\operatorname{HDF}$ (neona \\
\hline $\begin{array}{l}\text { DRR177025 } \\
\end{array}$ & CNhi10816_GCT.experiment & Negative control & NA & $20 \mid \mathrm{NA}$ & Homo sapiens & FANTOM6 project & PRJDB7993 & 14536073 & 47 CAGE & ASO_NC_A & HDF (neon \\
\hline DRR177024 & CNhi10816_GCG.experiment & $\begin{array}{l}\text { Targeted } \\
\text { Nepative }\end{array}$ & NA & 21 SOCS2-AS1 & Homo sapiens & FANTOM6 project & $\begin{array}{l}\text { PRJDB7993 } \\
\text { PRID7993 }\end{array}$ & $\begin{array}{l}11668511 \\
13698916\end{array}$ & $\begin{array}{l}47 \text { CAGE } \\
\text { A7CAGE }\end{array}$ & ASO_G0246985_AD_04 & HDF (neor \\
\hline DRR177022 & 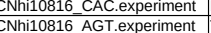 & $\begin{array}{l}\text { Negative control } \\
\text { Targeted }\end{array}$ & $\begin{array}{l}\text { NAA } \\
\text { NA }\end{array}$ & 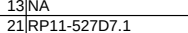 & $\begin{array}{l}\text { Homo sapiens } \\
\text { Homo sapiens }\end{array}$ & $\begin{array}{l}\text { FANTOM6 project } \\
\text { FANTOM6 project }\end{array}$ & $\begin{array}{l}\text { PRJJB79993 } \\
\text { PRJDB7993 }\end{array}$ & $\begin{array}{l}13699916 \\
15472147\end{array}$ & $\begin{array}{l}\text { 477CAGE } \\
47 \text { CAGE }\end{array}$ & $\begin{array}{l}\text { ASO_NC_B B } \\
\text { ASO G0225554 AD } 06\end{array}$ & $\begin{array}{l}\text { HDD (neonatat) } \\
\text { HDF (neoonata) }\end{array}$ \\
\hline $\begin{array}{l}\text { DRR177022 } \\
\text { DRR1702 }\end{array}$ & CNhi10816_AG_ACG.expereriment & $\begin{array}{l}\text { tagected } \\
\text { Targeted }\end{array}$ & NA & 20 EMC7 & Homo sapiens & $\begin{array}{l}\text { FANTOMG6 project } \\
\text { FANOMEct }\end{array}$ & 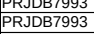 & 17175331 & $\begin{array}{l}47 C A C E \\
47 \text { CAGE }\end{array}$ & SiRNA_s32293 & $\begin{array}{l}\text { HoD (neonatal) } \\
\text { HDF (neonatat) }\end{array}$ \\
\hline & & Negative control & NA & 20 NA & & & PRJDB7993 & 19313579 & 47 CAGE & SiRNA_NC_2 & $\begin{array}{l}\text { HLFF (neounataf) } \\
\text { HDF (neonata) }\end{array}$ \\
\hline DRP176019 & CNhi10456_ACC.experiment & \begin{tabular}{|l|l|} 
Targeted \\
\end{tabular} & NA & 1) TUG1 & Homo sapiens & FANTOM6 project & PRJDB7993 & 18721025 & 47 CAGE & ASO_G0253352 & HDF (neonat \\
\hline DRR176018 & CNhi10455_TAC.experiment & Targeted & NA & 1AC017048.4 & Homo sapiens & FANTOM6 project & PRJDB7993 & 20519020 & 47 CAGE & 24577. & HDF (neor \\
\hline DRR176017 & CNNhi10455_GCT.experiment & $\begin{array}{l}\text { Targeted } \\
\text { Traneted }\end{array}$ & NA & 1/RP11-834C11.4 & Homo sapiens & FANTOM6 project & 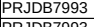 & 15467122 & $\begin{array}{l}47 \text { CAGE } \\
\text { A7CAGE }\end{array}$ & 0742 & $\begin{array}{ll}\text { HDF (neo } \\
\text { HDF (neo }\end{array}$ \\
\hline DRR176015 & $\begin{array}{c}\text { CNhi1i00555GCGGepperiment } \\
\text { CNhi10455 CAC.experiment }\end{array}$ & $\begin{array}{l}\text { Targeted } \\
\text { Targeted }\end{array}$ & $\begin{array}{l}\text { NA } \\
\text { NA }\end{array}$ & $\begin{array}{l}\text { 1LLINC00938 } \\
1 \text { FTX }\end{array}$ & $\begin{array}{l}\text { Homo sapiens } \\
\text { Homo sapiens }\end{array}$ & $\begin{array}{l}\text { FANTOM6 project } \\
\text { FANOM6 project }\end{array}$ & $\begin{array}{l}\text { PRJJB79993 } \\
\text { PRJDB7993 }\end{array}$ & $\begin{array}{ll}13335250 \\
13079333\end{array}$ & $\begin{array}{l}\text { 477CAGE } \\
47 \text { CAGE }\end{array}$ & 30590 & 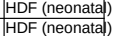 \\
\hline DRR1760 & CNhi104045 AGT.experiment & $\begin{array}{l}\text { Targected } \\
\text { Targeted }\end{array}$ & NA & 1EMX2OS & Homo sapiens & $\begin{array}{l}\text { FANTOM6 project } \\
\text { FANT }\end{array}$ & PRJDB7993 & 18755851 & 477CAGE & 9847 & $\operatorname{HDF}(n)$ \\
\hline URRILOUIS & & Targeted & NA & 1.SNHG18 & & FANTOM6 project & PRJDB7993 & 14574960 & 47 CAGE & 7786 & $F(1$ \\
\hline DRF & ACC.experiment & $\begin{array}{l}\text { Targeted } \\
\end{array}$ & NA & 1 FGD5-AS1 & Homo sapiens & FANTOM6 project & PRJDB7993 & 14714264 & 47CAGE & SSO_G0225733_04 & HDF (neona \\
\hline DRR1 & GCT.experiment & Targeted & NA & 22 LINC00630 & Homo sapiens & FANTOM6 project & PRJDB7993 & 11433567 & 47 CAGE & gRNA_blast5_LINCO & dHDF (neor \\
\hline DRR & GCG.experiment & Negative control & NA & 22 NA & Homo sapiens & FANTOM6 project & $\begin{array}{l}\text { PRJDB7993 } \\
\text { PRID7993 }\end{array}$ & 11670939 & $\begin{array}{l}47 \text { CAGE } \\
\text { A7CAGE }\end{array}$ & SGRNA_NC_gRNA1 & HDF (n) \\
\hline DRR176952 & 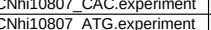 & $\begin{array}{l}\text { Negative control } \\
\text { Negative control }\end{array}$ & $\frac{\text { NA }}{\text { NA }}$ & $\begin{array}{l}22 \mathrm{NA} \\
22 \mathrm{NA}\end{array}$ & $\begin{array}{l}\text { Homo sapiens } \\
\text { Homo sapiens }\end{array}$ & $\begin{array}{l}\text { FANTOM6 project } \\
\text { FANTOM6 project }\end{array}$ & $\begin{array}{l}\text { PRJJB79993 } \\
\text { PRJDB7993 }\end{array}$ & $\begin{array}{l}\frac{15710645}{11254028} \\
-\end{array}$ & $\begin{array}{l}\text { 477CAGE } \\
47 \text { CAGE }\end{array}$ & $\begin{array}{l}\text { SgRNA_dCas9 } \\
\text { gRRNA NC GRNA2 }\end{array}$ & \\
\hline $\begin{array}{l}\text { URRI6952 } \\
\text { DRR176951 }\end{array}$ & CNhi10807 AGT.experiment & Negative control & NA & $\begin{array}{ll}22 \mathrm{NA} A \\
22 \mathrm{NA}\end{array}$ & $\begin{array}{l}\text { Homo saplens } \\
\text { Homo sapiens }\end{array}$ & $\begin{array}{l}\text { FANTOMG6 project } \\
\text { FANTOMect }\end{array}$ & $\begin{array}{l}\text { PRJDERT993 } \\
\text { PRDB7993 }\end{array}$ & $\frac{1115442080}{17996960}$ & $\begin{array}{l}\text { 47CACAE } \\
47 C A G E \\
\end{array}$ & gRNA_ blast5 control & $\begin{array}{l}\text { HDFF neon } \\
\text { HDF (neon }\end{array}$ \\
\hline DRR. & CNhi100807_ACG.expperiment & Targeted & NA & $\begin{array}{l}\text { 22 INA } 2 \text { LINC00630 } \\
\end{array}$ & Homo sapiens & FANTOM6 project & PRJDB7993 & 15088601 & 47 CAGE & GRNA_blast5_LINCO063 & 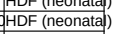 \\
\hline & CNhilo807_ACC.experiment & Negative control & NA & $222 \mathrm{NA}$ & Homo sapiens & FANTOM6 project & PRJDB7993 & 17414905 & 47 CAGE & sgRNA_NC & $\operatorname{HDF}(n$ \\
\hline $\begin{array}{l}\text { DRR176948 } \\
\text { DR170893 }\end{array}$ & CNhi10805_TAC.experiment & Negative control & NA & 19 NA & Homo sapiens & FANTOM6 project & PRJDB7993 & 18654719 & 47CAGE & ASO_NC_B & HDF (neo \\
\hline $\begin{array}{l}\text { DR176883 } \\
\text { DRR176882 }\end{array}$ & $\begin{array}{c}\text { CNhi100899_CAC.experiment } \\
\text { NNhi110789 ATG.experiment }\end{array}$ & $\frac{\text { Negative control }}{\text { Negative control }}$ & NA & $\frac{16 \text { NA }}{15 \mathrm{NA}}$ & $\begin{array}{l}\text { Homo sapiens } \\
\text { Homo saniens }\end{array}$ & $\begin{array}{l}\text { FANTOM6 project } \\
\text { FANOM6 project }\end{array}$ & PRJDB79993 & $\frac{14216151}{2335688}$ & $\begin{array}{l}47 \text { CAGE } \\
47 \text { CAGE }\end{array}$ & 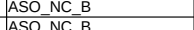 & $\frac{D F \text { (neon }}{\mathrm{DF} \text { (neon }}$ \\
\hline DRR176881 & & Targeted & NA & 16 CTD-2319112.4 & Homo sapiens & FANTOM6 project & 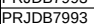 & 15157624 & 47CACE & $\frac{A S O}{A S O}$ G0267248 AD 07 & $\operatorname{DDFF(1)}$ \\
\hline DRR176880 & CNhi10789_ACG.experiment & Negative control & NA & $15 \mathrm{NA}$ & Homo sapiens & FANTOM6 project & PRJDB7993 & 20850749 & 47CAGE & SSO_Untreat & UDF (neoona \\
\hline $\begin{array}{l}\text { DRR176879 } \\
\text { D1689 }\end{array}$ & experiment & Targeted & NA & 15LINC00674 & Homo sapiens & FANTOM6 project & PRJDB7993 & 20924093 & 477CAGE & 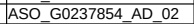 & \\
\hline & experiment & Targeted & & & Homo sapiens & FANTOM6 project & PRJDB79993 & 17561858 & 47 CAGE & $\overline{\mathrm{OG}}$ & DDFO \\
\hline $\begin{array}{l}\text { DRR } \\
\text { DPR }\end{array}$ & GCT.experiment & Targeted & NA & $\begin{array}{l}15 \text { CTD-2006C1.2 } \\
\end{array}$ & Homo sapiens & FANTOM6 project & $\begin{array}{l}\text { PRJDB79933 } \\
\text { DDD7002 }\end{array}$ & 15564975 & 47 CAGE & 665 C6D_04 & $\frac{D F(1}{D F(1)}$ \\
\hline $\begin{array}{l}\text { DR176876 } \\
\text { DRR176811 }\end{array}$ & CNhi100788_GCG.experiment & larageted & $\frac{\text { NA }}{\text { NA }}$ & 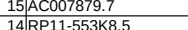 & Homo sapiens & FANTOM6 project & $\begin{array}{l}\text { PRJDB/9993 } \\
\text { PRDBR7993 }\end{array}$ & 15281196 & $\begin{array}{l}44 \text { CAGE } \\
47 \text { CAGE } \\
\end{array}$ & 11573 AD 04 & IDF (neo \\
\hline DPR16811 & AGT.experiment & larageted & NAT & 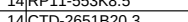 & & 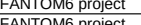 & 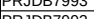 & 105442469 & 77CAOE & 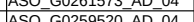 & HDF (ned \\
\hline 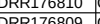 & S.expermiment & $\begin{array}{l}\text { I Iargeted } \\
\text { Taraeted }\end{array}$ & $\mathbb{N A}$ & 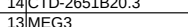 & $\begin{array}{l}\text { Homo saperens } \\
\text { Homo saing }\end{array}$ & $\begin{array}{l}\text { FANTOM6 project } \\
\text { FANTOM6 project }\end{array}$ & 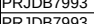 & (200609022 & $\begin{array}{l}47 C A G E \\
47 C A G E\end{array}$ & 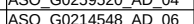 & $\begin{array}{l}\text { HDF (neor } \\
\text { HDF (neor }\end{array}$ \\
\hline $\begin{array}{l}\text { DRR16809 } \\
\text { DRR176808 }\end{array}$ & $\begin{array}{l}\text { experiment } \\
\text { experiment }\end{array}$ & Negated & $\mathrm{NA}$ & & $\begin{array}{l}\text { Homo saplens } \\
\text { Homo sapiens }\end{array}$ & 6 project & PRJDB7993 & 21301310 & $\begin{array}{l}47 \text { CALE } \\
47 \text { CAGE }\end{array}$ & ASO Untreat & HDF (I \\
\hline DRR176807 & NNhilo765_GCT.experiment & Targeted & NA & 14 CTD-2651B20.3 & Homo sapiens & FANTOM6 project & PRJDB7993 & $\begin{array}{l}14792847 \\
\end{array}$ & $\begin{array}{l}47 \text { CAGE } \\
47 \mathrm{CGCE}\end{array}$ & ASO_G0259520_AD_04 & HDF (ned \\
\hline $\begin{array}{l}\text { DRR176806 } \\
\text { DR17005 }\end{array}$ & 65_GCG.experiment & Targeted & NA & 13/RP11-527D7.1 & Homo sapiens & 16 project & $\begin{array}{l}\text { PRJDB79933 } \\
\text { DPID7092 }\end{array}$ & $\begin{array}{lll}11939892 \\
12704124\end{array}$ & $\begin{array}{l}47 \text { CAGE } \\
\text { TICAGE }\end{array}$ & ASO_G0225554_AD_06 & HDF (neo \\
\hline $\begin{array}{ll}\text { DRR176805 } \\
\text { DRR176804 }\end{array}$ & CAC.experiment & Negative control & NA & & Homo sapiens & FANTOM6 project & $\begin{array}{l}\text { PRJDB79933 } \\
\text { DPID7093 }\end{array}$ & $\begin{array}{r}13794124 \\
2004117 \\
\end{array}$ & $\begin{array}{l}\text { 47CAGE } \\
\text { A7CAGE }\end{array}$ & $\begin{array}{l}\text { ASO_NC_B B } \\
\text { ASO Lipo }\end{array}$ & $\begin{array}{ll}\text { HDF (neo } \\
\text { HDF (neo }\end{array}$ \\
\hline $\begin{array}{ll}\text { DRR16804 } \\
\text { DRR176739 }\end{array}$ & 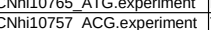 & $\begin{array}{l}\text { Negative control } \\
\text { Targeted }\end{array}$ & NA & $\begin{array}{l}13 \text { NA } \\
17 \text { RP11-611E13.2 }\end{array}$ & $\begin{array}{l}\text { Homo saplens } \\
\text { Homo sapiens }\end{array}$ & $\begin{array}{l}\text { FANTOM6 project } \\
\text { FANTOM6 project }\end{array}$ & $\begin{array}{l}\text { PRJUB/9993 } \\
\text { PRJDB7993 }\end{array}$ & $\begin{array}{l}2004411 \\
19425424 \\
\end{array}$ & $\begin{array}{l}\text { 47CAGE } \\
47 \text { CAGE }\end{array}$ & $\begin{array}{ll}\text { ASO_LO G02 } \\
\text { ASO G02515 AD } 03\end{array}$ & $\begin{array}{l}\text { Hof (neona } \\
\text { HDF (neona }\end{array}$ \\
\hline DRR176738 & CNhi10757_ACC.experiment & Targeted & NA & 14RP5-886K2.3 & Homo sapiens & FANTOM6 project & PRJDB7993 & 163948484 & $\begin{array}{l}47 C A C E \\
47 \text { CAGE }\end{array}$ & ASO_G0236810_AD_06 & HDF (neonat \\
\hline DRR176737 & periment & $\begin{array}{l}\text { Experiment } \\
\text { control }\end{array}$ & NA & 12|MALAT1 & Homo sapiens & FANTOM6 project & PRJDB7993 & 15512261 & 47 CAGE & LAT1 & \\
\hline 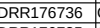 & & & & 12ERVKK13-1 & & & & 14044030 & & & \\
\hline DRR176735 & SCG.experiment & Targeted & $\mathrm{NA}$ & 12 TP53TG1 & Homo sapiens & FANTOM6 project & PRJDB7993 & 12132651 & 477CAGE & 165 AD 04 & HDF (neo \\
\hline & experiment & Targeted & NA & 12 TUC & Homo sapiens & project & & 14718914 & 47 CAGE & $A D_{2} 08$ & \\
\hline & & Targeted & NA & 12:ME & Homo sapiens & $c t$ & & 111278052 2 & 47CAGE & 14548 AD_09 & \\
\hline & $\begin{array}{l}\text { 40_AGT.experiment } \\
31 \text { TACexperiment }\end{array}$ & $\begin{array}{l}\text { Targeted } \\
\text { Targeted }\end{array}$ & INA & $\begin{array}{l}1 \text { 1/FX } \\
12 \text { AC017048.4 }\end{array}$ & $\begin{array}{l}\text { Homo sapelins } \\
\text { Homo sains. }\end{array}$ & 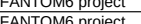 & (2) & 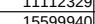 & (47CAGE & 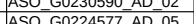 & $\begin{array}{l}\frac{H D F F \text { (neo }}{H D F(n e o} \\
\text { HDF }\end{array}$ \\
\hline & GCTexperiment & Targeted & $\mathrm{NA}$ & $21 \mathrm{N1} 13.3$ & Homo sapiens & project & PRJ & 125 & & 07 & \\
\hline DR176665 & 1 GCG experiment & TTareted & NA & 12 RAB30-AS1 1 & Homo saniens & 6 project & & 11841344 & 47 CAGE & 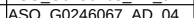 & \\
\hline & experiment & Targeleu & $\mathrm{NA}$ & & Homo sapiens & 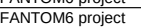 & PRJDB7993 & 15838681 & 47 CAGE & ASO & HDF $(n$ \\
\hline DRF & experiment & Targeted & NA & \begin{tabular}{ll|l}
12 RAB $30-A$ \\
\end{tabular} & Homo sapiens & FANTOM6 project & PRJDB7993 & 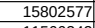 & 47.CAGE & ASO_G & HDF (n) \\
\hline & t. & & NA & 12|FGD5-AS1 & Homo sapiens & 16 project & 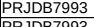 & & $\begin{array}{ll}47 \text { CAGE } \\
7 C A C 5\end{array}$ & SO_G0225733_AD_06 & \\
\hline 661 & 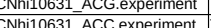 & $\begin{array}{l}\text { Targeted } \\
\text { TTaraeted }\end{array}$ & NAA & $\begin{array}{ll}\frac{12 \mathrm{DNN}}{12 \mathrm{DP1}} \\
12 \mathrm{RP1}\end{array}$ & $\begin{array}{l}\text { Homo sapiens } \\
\text { Homo saniens }\end{array}$ & $\begin{array}{l}\text { FANTOM6 project } \\
\text { FANTOM6 project }\end{array}$ & $\begin{array}{l}\text { PRJD } \\
\text { PRJD }\end{array}$ & $\frac{131}{162}+2>0$ & 年 470 & $\begin{array}{l}\text { G02306630AD_ } 10 \\
\text { G0250742 AD } 05\end{array}$ & $\begin{array}{l}\text { HDF (neo } \\
\text { HDF neo }\end{array}$ \\
\hline 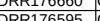 & L & larageted & (n) & t5it1.4 & Homb sponins & 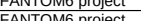 & PR & 1421110909 & 470 & G0242627302 & HDDF (neoun \\
\hline 6. 65954 & GCG experiment & $\begin{array}{l}\text { Trargeted } \\
\text { Taraeted }\end{array}$ & NA & 0162 & $\begin{array}{l}\text { Homo saplens } \\
\text { Homo saniens }\end{array}$ & 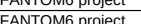 & & 1432113999 & $\frac{47 \sqrt{4}}{476}$ & $\frac{15-02}{68202}$ & (neoric \\
\hline DRR & $\begin{array}{l}\text { experiment } \\
\text { experiment }\end{array}$ & $\begin{array}{l}\text { Lalageteu } \\
\text { Targeted } \\
\end{array}$ & NA & $\begin{array}{l}\text { OSLL16.2 } \\
\text { AS1 }\end{array}$ & $\begin{array}{l}\text { Homo saplens } \\
\text { Homo sapiens }\end{array}$ & FANT & $\begin{array}{l}P R^{2} \\
P R^{2}\end{array}$ & $\frac{1024}{974}$ & $\begin{array}{l}47 C A C E \\
47 \text { CAGE }\end{array}$ & ASO_G0248508_10 & HDF (neor \\
\hline & experiment & Targeted & NA & & Homo sapiens & project & PRJDB7993 & 95 & 47 CAGE & 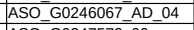 & HDF (neo \\
\hline 591 & AGT.experiment & Targeted & NA & 10 & Homo sapiens & 6 6 project & $\begin{array}{lll}P R \\
P R\end{array}$ & $\begin{array}{r}13409484 \\
1326209 \\
\end{array}$ & 47CAGE & ASO_60247572_09 & HDF (neon \\
\hline & experiment & Targented & NA & $\begin{array}{l}10 \mid R P 11-660016.2 \\
\end{array}$ & Homo sapiens & 6 pro & PRJD & 13362869 & 47/CAGE & ASO_60254682___6 & $\operatorname{HDF}(\mathrm{ne}$ \\
\hline & experiment & Targeted & NA & 1018017.3 & Homo sapiens & $6 \mathrm{prc}$ & PRJD & & $4 \pi] C$ & SSOGG & \\
\hline & TAC.experiment & Targeted & NA & 9038 7938 & Homo sapiens & opro & PRJD & & 4796 & D-1 & \\
\hline & nit & largelea & NA & 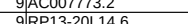 & $\begin{array}{l}\text { Homo Sapiens } \\
\text { Homo sains }\end{array}$ & EAI & & & 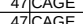 & & \\
\hline & 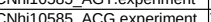 & Targe & $\frac{N A}{N A}$ & 年 & 棐 & & & & & & \\
\hline 6520 & experiment & Targeted & NA & $1-6 N 17.4$ & Homo & M6 pro & PRJDB & 14121901 & & So_c & \\
\hline
\end{tabular}


Table_S3 Meatadata for single cell analysis and FANTOM6 kmer research

\begin{tabular}{|c|c|c|c|c|c|c|c|c|c|c|c|c|}
\hline 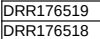 & 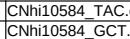 & $\begin{array}{l}\text { cexperiment } \\
\text { Tie.experiment } \\
\end{array}$ & $\begin{array}{l}\text { Targated } \\
\text { Targated }\end{array}$ & $\frac{N A}{N A}$ & $\begin{array}{l}\text { 9LLNC006667 } \\
\text { 9RPP11-6N17.4 }\end{array}$ & $\begin{array}{l}\text { Homo sapiens } \\
\text { Homo sapiens }\end{array}$ & \begin{tabular}{|l|l|} 
FANTOM6 project \\
FANTOM6 project
\end{tabular} & \begin{tabular}{|l} 
PRJDB79933 \\
PRJDB7993
\end{tabular} & \begin{tabular}{|l|}
13909884 \\
11566175 \\
\end{tabular} & $\begin{array}{l}47 \text { CAGEE } \\
47 \text { CAGE }\end{array}$ & 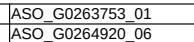 & $\frac{\mid H D F \text { (neona }}{\text { HDF (neona }}$ \\
\hline & & G.experiment & Targeted & NA & 9RP11-95P2.1 & Homo sapiens & FANTOM6 project & PRJDB79993 & 11983984 & 47 CAGE & & HDF (neonata) \\
\hline $\begin{array}{ll}\text { DRR176516 } \\
\end{array}$ & CNhi10584_CAC. & c.experiment & Targeted & NA & \begin{tabular}{|l|l|} 
RP11-95P2.1 \\
\end{tabular} & Homo sapiens & FANTOM6 project & PRJDB7993 & 17721134 & 47CAGE & ASO_G0262468_04 & \\
\hline \begin{tabular}{|l|l|} 
DRR176451 \\
DRR176450
\end{tabular} & $\begin{array}{l}\text { CNhi10544_AGT. } \\
\text { CNhi10544ACG }\end{array}$ & $\begin{array}{l}\text { T.experiment } \\
\text { S.experiment }\end{array}$ & $\begin{array}{l}\text { Targeted } \\
\text { Targated }\end{array}$ & $\frac{N A}{N A}$ & $\begin{array}{l}\text { 5KTN1-AS1 } \\
\text { 6R11-422J8.1 }\end{array}$ & \begin{tabular}{|l|} 
Homo sapiens \\
Homo sapiens
\end{tabular} & \begin{tabular}{|l} 
FAANOM6 project \\
FANTOM6 rojoct
\end{tabular} & \begin{tabular}{|l|l|} 
RRJDD7993 \\
PRJB7993
\end{tabular} & \begin{tabular}{|l|}
12690095 \\
12876174 \\
\end{tabular} & $\begin{array}{l}47 \text { CAGE } \\
47 \text { CAGE }\end{array}$ & $\frac{\text { ASO_G0186615_09 }}{\text { ASO G0233621 } 01}$ & $\frac{\text { HDF (neonatat) }}{\text { HDF (neonatat) }}$ \\
\hline $\begin{array}{l}\text { DRR16450 } \\
\text { DRR176449 }\end{array}$ & \begin{tabular}{|l} 
CNNhi10544_ACG \\
CNhi10544ACC.
\end{tabular} & $\begin{array}{l}\text { S.eeperiment } \\
\text { C.experiment }\end{array}$ & $\begin{array}{l}\text { Iargeled } \\
\text { Targeted } \\
\end{array}$ & $\mathrm{NA}$ & 5/CATG00000090305.1 & $\begin{array}{l}\text { Homo sapiens } \\
\text { Homo sapiens }\end{array}$ & $\begin{array}{l}\text { FANIOM6 project } \\
\text { FANTOM6 project }\end{array}$ & 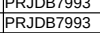 & \begin{tabular}{|l|}
12861614 \\
14512776 \\
\end{tabular} & $\begin{array}{l}4 \pi \text { CAGE } \\
477 \text { CAGE }\end{array}$ & $\begin{array}{ll}\mathrm{ASO} \mathrm{SOCO}_{\mathrm{CO}} \\
\mathrm{A} \mathrm{CO}\end{array}$ & $\begin{array}{l}\text { HDF (neona } \\
\text { HDF (neona }\end{array}$ \\
\hline DRR176907 & & & & NA & 16 AC 009005.2 & & FANTOM6 project & PRJDB7993 & 15652189 & 47 CAGE & SO_G0267751_AD_02 & $\begin{array}{l}\text { HDVF (neona } \\
\text { HDF (neona }\end{array}$ \\
\hline DRR176906 & CNhi10792 CAC. & c.experiment & Targeted & $\mathrm{NA}$ & 15. DANCR & Homo sapiens & FANTOM6 project & \begin{tabular}{|l|l|} 
PRJDB 7993 \\
\end{tabular} & 14684033 & 477CAGE & ASO G0226950 04 & HDF (neon \\
\hline \begin{tabular}{|l} 
RRR176905 \\
\end{tabular} & CNhi10792_ATG. & S.experiment & Targeted & NA & 16 ZNF730 & Homo sapiens & FANTOM6 project & PRJDB7993 & 21340913 & 477CAGE & ASO_G0267886_AD_04 & HDF (neon \\
\hline \begin{tabular}{|l|l|} 
DRR176904 \\
DRR176093
\end{tabular} & $\begin{array}{l}\text { CNhi10792_AGT. } \\
\text { CNhbi10292ACG }\end{array}$ & T.experiment & Targeted & NA & 15LLINC00674 & Homo sapiens & FANTOM6 project & $\begin{array}{l}\text { PRJDB79933 } \\
\text { PRID7993 }\end{array}$ & \begin{tabular}{|l}
19131885 \\
2208137
\end{tabular} & $\begin{array}{l}\text { 47CAGE } \\
\text { A7CAGE }\end{array}$ & ASO_G0237854_AD_04 & HDF (neon \\
\hline $\begin{array}{l}\text { DRR1169903 } \\
\text { DRR176902 }\end{array}$ & \begin{tabular}{|l} 
CNhi10792_ACG. \\
CNhi10792ACC.
\end{tabular} & G.experiment & $\begin{array}{l}\text { Negative control } \\
\text { Targeted }\end{array}$ & NA & $\frac{15 \text { NA }}{16 \text { CTC-559E9.6 }}$ & \begin{tabular}{|l} 
Homo sapiens \\
Homo sapiens
\end{tabular} & $\begin{array}{l}\text { FANTOM6 project } \\
\text { FANTOM6 project }\end{array}$ & $\begin{array}{l}\text { PRJDB79993 } \\
\text { PRJDB7993 }\end{array}$ & $|220841373|$ & $\begin{array}{l}\text { 477CAGE } \\
47 \text { CAGE }\end{array}$ & 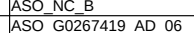 & $\begin{array}{l}\text { HDF (neon } \\
\text { HDF neon }\end{array}$ \\
\hline 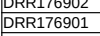 & 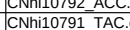 & $\begin{array}{l}\text { C.experiment } \\
\text { cexperiment }\end{array}$ & $\begin{array}{l}\text { Irargeled } \\
\text { Targeted }\end{array}$ & NA & $\frac{15}{15 P I T P N A-A S 1}$ & $\begin{array}{l}\text { Homo Sapiens } \\
\text { Homo sapiens }\end{array}$ & $\begin{array}{l}\text { FANAOM6 pijoct } \\
\text { FANTOM6 project }\end{array}$ & 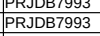 & & $\begin{array}{l}4 \pi \text { CAGE } \\
477 \text { CAGE }\end{array}$ & 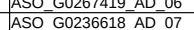 & \begin{tabular}{|l} 
HDF (neon \\
HDF (neon
\end{tabular} \\
\hline & & T.experiment & & NA & & & & PRJDB7993 & 16491490 & & 50 Go265458 AD 03 & $\begin{array}{l}\text { HDFF (neon } \\
\text { HDF neon }\end{array}$ \\
\hline DRR176835 & $\begin{array}{l}\text { CNhi10783 ATG. } \\
\end{array}$ & S.experiment & Targeted & $\mathrm{NA}$ & 16 CTC-559E9.6 & Homo sapiens & FANTOM6 project & \begin{tabular}{|l|l|} 
PRJDB 7993 \\
\end{tabular} & 20653423 & 477CAGE & ASO_G0267419AD_04 & \\
\hline 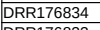 & CNhi10783_AGT. & T.experiment & Targeted & NA & 17 AC013394.2 & Homo sapiens & FANTOM6 project & PRJDB7993 & 16016295 & 47 CAGE & ASO_G0272888_AD_10 & \\
\hline 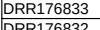 & \begin{tabular}{|l} 
CNhi10783_ACG. \\
CNhi10783ACC.
\end{tabular} & G.experiment & $\begin{array}{l}\text { Targeted } \\
\text { Taretes }\end{array}$ & NA & 17 AC013394.2 & Homo sapiens & FANTOM6 project & $\begin{array}{l}\text { PRJDB79933 } \\
\text { PRID7993 }\end{array}$ & \begin{tabular}{|l|l|}
22688667 \\
2025737
\end{tabular} & $\begin{array}{ll}47 C A G E \\
77 C A G E\end{array}$ & ASO_G0272888_AD_10 & $\begin{array}{l}\text { HDF (ne } \\
\text { HDF (ne }\end{array}$ \\
\hline $\begin{array}{l}\text { DRR116832 } \\
\text { DRR176831 }\end{array}$ & \begin{tabular}{|l} 
CNhi10783_ACC. \\
CNhi10782 TAC.
\end{tabular} & C.experiment & $\begin{array}{l}\text { Targeted } \\
\text { Targeted }\end{array}$ & NA & $\frac{16 \text { ZNNFT30 }}{17 \text { CTD-3018017.3 }}$ & \begin{tabular}{|l} 
Homo sapiens \\
Homo sapiens
\end{tabular} & $\begin{array}{l}\text { FANTOM6 project } \\
\text { FANTOM6 project }\end{array}$ & $\begin{array}{l}\text { PRJDB79993 } \\
\text { PRJDB7993 }\end{array}$ & $20257377 \mid$ & $\begin{array}{l}\text { 477CAGE } \\
47 \text { CAGE }\end{array}$ & 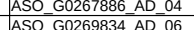 & $\frac{\mathrm{HDF}}{\mathrm{HDF}}$ \\
\hline & $\begin{array}{l}\text { CNnh10/8__IAC. } \\
\text { CNhi1078_GCT. }\end{array}$ & & Targeter & NA & 15 LINC00674 & $\begin{array}{l}\text { Homo sapiens } \\
\text { Homo sapiens }\end{array}$ & $\begin{array}{l}\text { FANTOM6 project } \\
\text { FANTOM6 project }\end{array}$ & $\begin{array}{l}\text { PRJJDRB } \\
\text { PR9933 } \\
\end{array}$ & 15740880 & & & \\
\hline DRR176829 & 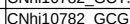 & Geperiment & $\begin{array}{l}\text { Targeteded } \\
\text { Targed }\end{array}$ & NA & 16 L INC00667 & 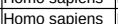 & & PRJDB7993 & & AGE & 0 & $\begin{array}{l}\text { HDF (neon } \\
\text { HDF neon }\end{array}$ \\
\hline DRR176828 & CNhi10782_CAC. & C.experiment & Negative control & $\mathrm{NA}$ & $15 \mathrm{NA}$ & Homo sapiens & FANTOM6 project & \begin{tabular}{|l|l|} 
PRJDB7993 \\
\end{tabular} & 15353713 & 47 CAGE & ASO_Lipo & $\begin{array}{l}\text { HDF (neon } \\
\text { HDF (neon }\end{array}$ \\
\hline 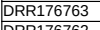 & CNhi10760_ACG. & G.experiment & Targeted & NA & 14/RP11-553K8.5 & Homo sapiens & FANTOM6 project & PRJDB7993 & $\begin{array}{l}17101439 \\
\end{array}$ & 47CAGE & ASO_G0261573_AD_06 & HDF (neon \\
\hline \begin{tabular}{|l|l|l|l|l|l} 
DRR176762 \\
DRR176761
\end{tabular} & \begin{tabular}{|l} 
CNhi10760_ACC. \\
CNhbi1759 TAC
\end{tabular} & C.experiment & \begin{tabular}{|l} 
Negative control \\
Tracted
\end{tabular} & NA & $133 \mathrm{NA}$ & Homo sapiens & FANTOM6 project & $\begin{array}{l}\text { PRJDB79993 } \\
\text { PRID7993 }\end{array}$ & \begin{tabular}{|l|}
20527193 \\
2308444 \\
\end{tabular} & $\begin{array}{l}47 \text { CAGE } \\
\text { A7CAGE }\end{array}$ & ASO_NC_B & HDF (neon \\
\hline 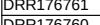 & 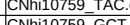 & C.experiment & $\begin{array}{l}\text { Targeted } \\
\text { Targated } \\
\end{array}$ & NA & $\begin{array}{l}\text { 14LLINC00059 } \\
14 \text { RP11-1738145 }\end{array}$ & $\begin{array}{l}\text { Homo sapiens } \\
\text { Homs }\end{array}$ & FANOM6 rojoect & $\begin{array}{l}\text { PRJDB79993 } \\
\text { PRJPR7993 }\end{array}$ & $\begin{array}{l}230800644 \\
203757\end{array}$ & A $A G E$ & 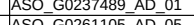 & HDF (neon \\
\hline $\begin{array}{l}\text { DRR176759 } \\
\text { DRR170 }\end{array}$ & $\begin{array}{l}\text { CNNhi10759_GCT. } \\
\text { CNhi10759 GCG }\end{array}$ & T.experiment & $\begin{array}{l}\text { Targeted } \\
\text { Targated }\end{array}$ & INA & 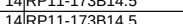 & H Homo sapens & $\begin{array}{l}\text { FANTOM6 project } \\
\text { FANTOM6 proiect }\end{array}$ & 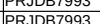 & $\frac{10211307}{125109}$ & (47CAGE & 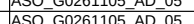 & HDF \\
\hline DRR176758 & $\begin{array}{l}\text { CNhi10759 } \\
\text { CNAC. }\end{array}$ & $\begin{array}{l}\text { Geexperiment } \\
\text { C.experiment }\end{array}$ & $\begin{array}{l}\text { argelea } \\
\text { Negative control }\end{array}$ & $\frac{N A}{N A}$ & $14 \mathrm{NA}$ & Homo sapiens & $\begin{array}{l}\text { FANIUNG6 project } \\
\text { FANTOM6 project }\end{array}$ & PRJDB7993 & \begin{tabular}{|l|}
12531999 \\
16338392
\end{tabular} & $\begin{array}{l}\text { 47CACAE } \\
47 C A G E \\
\end{array}$ & SSO NC B B $B$ & $\begin{array}{l}\text { HDF (neon } \\
\text { HDF neon }\end{array}$ \\
\hline DRR176757 & CNhi10759_ATG. & 5.experiment & Targeted & NA & 13|RP11-137L10.6 & Homo sapiens & FANTOM6 project & PRJDB7993 & 13426352 & 47 CAGE & ASO_G0221817_AD_08 & HDF (neon \\
\hline DRR176756 & CNhi10759_AGT. & T.experiment & Targeted & NA & 14 CTD-2651B20.3 & Homo sapiens & FANTOM6 project & PRJDB7993 & $\begin{array}{l}16115894 \\
159002\end{array}$ & 47 CAGE & ASO_G0259520_AD_08 & HDF $(n$ \\
\hline \begin{tabular}{|l|l|} 
DRR176691 \\
DPR176660
\end{tabular} & CNhi10635_ACC. & c.experiment & \begin{tabular}{|l|} 
Negative control \\
Taten d
\end{tabular} & NA & $\begin{array}{l}12 \text { NA } \\
12 \text { SNHG18 }\end{array}$ & Homo sapiens & FANTOM6 project & $\begin{array}{l}\text { PRJDB79933 } \\
\text { PRID7993 }\end{array}$ & 15891026 & $\begin{array}{ll}47 C A G E \\
77 C A G E\end{array}$ & ASO_NC_A & HDF \\
\hline $\begin{array}{l}\text { DRR116660 } \\
\text { DRR176689 }\end{array}$ & $\begin{array}{l}\text { CNNhi10634_TAC. } \\
\text { CNhi10634 GCT. }\end{array}$ & C.experiment & $\begin{array}{l}\text { Targeted } \\
\text { Targated } \\
\end{array}$ & $\frac{\mathrm{NA}}{\mathrm{NA}}$ & $\begin{array}{l}\frac{12 \text { SNHG18 }}{12 \text { DNMOS }} \\
\text { 19. }\end{array}$ & \begin{tabular}{|l|} 
Homo sapiens \\
Homo sapiens
\end{tabular} & $\begin{array}{l}\text { FANTOM6 project } \\
\text { FANTOM6 project }\end{array}$ & $\begin{array}{l}\text { PRJJB79993 } \\
\text { PRJDB7993 }\end{array}$ & $\begin{array}{l}17251215 \\
1297795 \\
\end{array}$ & $\begin{array}{l}\text { 477CAGE } \\
47 \text { CAGE }\end{array}$ & $\begin{array}{l}\text { ASO_OSO2500786_AD_ } 10 \\
\text { ASO G0230630 AD } 10\end{array}$ & \begin{tabular}{|l}
$\operatorname{HDF}(r)$ \\
$\operatorname{HDF}(1)$
\end{tabular} \\
\hline 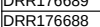 & CNhi10634_GCT. & T.experiment & $\begin{array}{l}\text { Targeted } \\
\text { Targated } \\
\end{array}$ & NAA & 年 & thomo sapens & $\begin{array}{l}\text { FANIUNG6 project } \\
\text { FANTOM6 project }\end{array}$ & 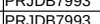 & $\frac{1296795}{165894}$ & (47CAGE & $\begin{array}{l}\text { ASO_G0230630_AD_10 } \\
\text { ASO G0250786_D } 10\end{array}$ & HDF \\
\hline DRR176687 & CNhi10634 CAC. & 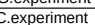 & Targeted & $\frac{N A}{N A}$ & 12 RP11-834C11.4 & $\begin{array}{l}\text { Homosapiens } \\
\text { Homo sains }\end{array}$ & FANTOM6 project & PRJDB7993 & 145853182 & $\begin{array}{l}47 \text { CAGE } \\
\text { 47 CAG }\end{array}$ & 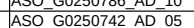 & $\frac{H D}{H D}$ \\
\hline DRR176686 & CNhi10634_ATG. & 5.experiment & Targeted & NA & 12 EMX2 & Homo sapiens & FANTOM6 project & PRJDB7993 & 14043996 & 477CAGE & ASO_G0229847_AD_04 & HDF ( \\
\hline DRR176685 & 34_AG & T.experiment & Targeted & NA & 12 RAB30-AS1 & Homo sapiens & FANTOM6 project & PRJDB7993 & 12568153 & 47CAGE & 246067_AD_09 & HDF $(n$ \\
\hline $\begin{array}{l}\text { DRR116664 } \\
\text { DRR176619 }\end{array}$ & \begin{tabular}{|l|} 
CNhh10634_ACG \\
CNhi10609GCT
\end{tabular} & $\begin{array}{l}\text { G.experiment } \\
\text { T.experiment }\end{array}$ & $\frac{\text { Negative control }}{\text { Targeted }}$ & NA & $\frac{12 \text { 12 NA }}{10 \text { LINC00959 }}$ & \begin{tabular}{|l} 
Homo sapiens \\
Homo saaiens
\end{tabular} & \begin{tabular}{|l|} 
FANTOM6 6 project \\
FANTOM6 proje
\end{tabular} & $\frac{\text { PRJDD79993 }}{\text { PRJDB7993 }}$ & \begin{tabular}{|l|}
136582888 \\
1256937
\end{tabular} & $\begin{array}{l}47 \text { CAGE } \\
477 \text { CAGE }\end{array}$ & ASO_NC_A & $\begin{array}{l}\text { HDF (neonal } \\
\text { HDF (neonat }\end{array}$ \\
\hline DRR176618 & & G.experiment & ITargeleu & NA & $10 R P 11-11011.12$ & Homo sapiens & FANTOM6 project & PRJDB7993 & 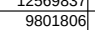 & & ASO GO255121 02 & \begin{tabular}{|l} 
HDFF (neonal \\
$\mathrm{HDF}$ (neonal
\end{tabular} \\
\hline DRR1 & CNhi10609_CAC. & $\begin{array}{l}\text { Se.experiment } \\
\text { C.experime }\end{array}$ & Negative control & & $11 \mathrm{NA}$ & Homo sapiens & FANTOM6 project & PRJDB7993 & 13965835 & 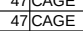 & ASO NC A & $\frac{\mid \text { HDF (neonal }}{\text { HDF (neonal }}$ \\
\hline \begin{tabular}{|l|l|} 
DRR176616 \\
DPR176615
\end{tabular} & CNhi10609_ATG. & s.experiment & Targeted & NA & 10|RP11-11011.12 & Homo sapiens & FANTOM6 project & PRJDB7993 & 9490839 & AAGE & ASO_G0255121 & \\
\hline \begin{tabular}{|l|l|} 
DRR176615 \\
\end{tabular} & CNhi1 & T.experiment & Targeted & & $\overline{10 \mathrm{ZNF} 73 \mathrm{C}}$ & Homo sapiens & FANTOM6 project & PRJDB7993 & 11403080 & $\begin{array}{lll}47 \mathrm{CAGE} \\
\end{array}$ & ASO_Co267886 & HDF (n \\
\hline 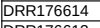 & CNhi1 & G.experiment & Targated & NA & 10/NR2F1-AS1 & Homo sapiens & FANTOM6 project & |PRJDB79993 & 96551859 & (77) CAGE & 237187_0 & HDF $(n$ \\
\hline 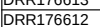 & CNhit & 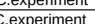 & Tagrated & NA & 11LINCO0938 & Homo sapiens & FANTOM6 project & PRJDB7993 & \begin{tabular}{|l|}
12044293 \\
13123402
\end{tabular} & $\begin{array}{l}4 \text { 4. } \\
7 \text { CAGE }\end{array}$ & ASO GO Ge273015 05 & \\
\hline DRR176547 & CNhit & & Neative control & NA & & Homo sapiens & FANTOM6 project & PRJT & 年 & 77 CAGE & ASO NCA A & \\
\hline DRR176546 & CNbi10588 & Copperiment & Neative control & NA & $9 \mathrm{NA}$ & Homo sapiens & FANTOM6 proiect & PRJBD7993 & 12751916 & CAGE & ASO NC A & $\begin{array}{l}\text { HDF (neon } \\
\text { HDF neon }\end{array}$ \\
\hline DRR176545 & CNhi10588 ATG. & 5.experiment & Negative control & $\mathrm{NA}$ & $9 \mathrm{NA}$ & Homo sapiens & FANTOM6 project & PRJDB7993 & $\mid 12309647$ & 47 CAAGE & $50=\mathrm{NC} A$ & HDF \\
\hline DRR176544 & CNhii & T.experiment & Negative control & NA & $9 \mathrm{NAA}$ & Homo sapiens & M6 project & PRJDB7993 & 13338104 & 477CAGE & ASO_NC_A & $\operatorname{HDF}(\mathrm{ne})$ \\
\hline $\begin{array}{l}\text { DRR116553 } \\
\text { DRR176542 }\end{array}$ & $\begin{array}{l}\text { CNNhi10588AACG. } \\
\text { CNhi10588 ACC }\end{array}$ & G.experiment & $\begin{array}{l}\text { Negative control } \\
\text { Negative control }\end{array}$ & NA & $\begin{array}{l}9 \text { NA } \\
9 \mathrm{NA}\end{array}$ & $\begin{array}{l}\text { Homo sapiens } \\
\text { Homo sapiens }\end{array}$ & $\begin{array}{l}\text { FANTOM6 project } \\
\text { FANTOM6 proje }\end{array}$ & $\begin{array}{l}\text { PRJDD79993 } \\
\text { PRJDB7993 }\end{array}$ & \begin{tabular}{|l|}
1115464909 \\
12921391
\end{tabular} & $\begin{array}{l}\text { 47/CAGE } \\
47 \text { CAGE }\end{array}$ & $\frac{A S O N N C A}{A S O N C A}$ & \begin{tabular}{|l} 
HDF neon \\
HDF neon
\end{tabular} \\
\hline DRR176541 & CNhi10587 TAC. & Cexperiment & $\begin{array}{l}\text { Experimen } \\
\text { control }\end{array}$ & NA & 9 MALAT1 & Homo sapiens & ect & PRJDB7993 & 12340215 & & ASO MALAT1 & \\
\hline DRR176540 & & G.experiment & Targeted & NA & $9 A \subset 009005.2$ & Homo sapiens & N6 project & & $\mid$ & 47 & 7751_07 & \\
\hline DRR: & CNhi1 & T.exper & Negative control & $\mathrm{NA}$ & 6 NA & Homo sapiens & FANTOM6 project & PRJDB7993 & 12686319 & 477CAGE & $\overline{A S O}=1$ & \\
\hline & & experrment & argeted & NA & 6IRP11-245M24.1 & Homo sapiens & FANTOM6 project & & 10659/29 & & 9563_04 & HDF $(1$ \\
\hline \begin{tabular}{|l|l|l|l|l} 
DRR17473 \\
\end{tabular} & CNhi10579_ACC. & C.experiment & \begin{tabular}{|l|} 
Negative control \\
Experimet
\end{tabular} & NA & 6 NA & Homo sapiens & FANTOM6 project & PRJDB7993 & 13086624 & 47.CAGE & ASO_NC_A & HDF (neone \\
\hline DRR176472 & CNhi105 & C.experiment & $\begin{array}{l}\text { Expermment } \\
\text { control }\end{array}$ & NA & 6 MALAT1 & Homo sapiens & FANTOM 6 project & PRJDB7993 & 14184428 & 47) CAGE & LAT1 & HDF (ne \\
\hline DRR & & T.experiment & \begin{tabular}{|l|} 
Negative control \\
Tat
\end{tabular} & NA & $6 \pi$ & Homo sapiens & FAl & & 655 & 477CAGE & & \\
\hline & CNhi- & & $\begin{array}{l}\text { Fargeted } \\
\text { argated }\end{array}$ & $\frac{N A}{N A}$ & $\begin{array}{l}\text { 6PIIPNA-AS1 } \\
6 \text { LINC0263 }\end{array}$ & Homo sapiens & 6 project & $\begin{array}{l}\text { PRJDB79993 } \\
\text { PRJP7993 }\end{array}$ & \begin{tabular}{|c|}
15149146 \\
13753762
\end{tabular} & $\begin{array}{l}\text { 47CAGE } \\
47 C A G E\end{array}$ & $\begin{array}{ll}6618=07 \\
58233\end{array}$ & \\
\hline DRR176469 & CNhi10578_CAC. & C.experiment & $\frac{\mid \text { Targeted }}{\text { Experiment }}$ & & b LLINCUOLZ63 & Homo sapiens & 66 project & DETY93 & & & $5823=03$ & HOF $($ I \\
\hline DRR176468 & CNhi1 & S.experiment & $\begin{array}{l}\text { control } \\
\text { contin }\end{array}$ & NA & & Homo sapiens & project & & 13082652 & 47 CAGE & ASO & HDF $(\mathrm{ne}$ \\
\hline \begin{tabular}{|l} 
DRR1164603 \\
DRR176402
\end{tabular} & \begin{tabular}{|l|} 
CNhhi10538_ACG \\
CNhi10538ACC
\end{tabular} & G.exp & tive control & NA & $\begin{array}{l}66 \mathrm{ZNF}^{2} \\
5 \mathrm{NA}\end{array}$ & Homo sapiens & \begin{tabular}{|l|} 
FAATOM6 project \\
FNTOM6 pojoet
\end{tabular} & \begin{tabular}{|l|l|} 
PRJDB79993 \\
PRJB7993
\end{tabular} & \begin{tabular}{|c|}
12459693 \\
1323978
\end{tabular} & $\begin{array}{l}47 \text { CAGE } \\
47 \text { CAGE }\end{array}$ & $\begin{array}{l}\text { ASO_G02344 } \\
\text { ASO N A A }\end{array}$ & $\begin{array}{l}H D F(n \\
H D F(n\end{array}$ \\
\hline DRR1 & CNhi1 & Cexper & $\begin{array}{l}\text { Experiment } \\
\text { control }\end{array}$ & NA & 5 MALAT & Homo sapiens & project & PRJD & 150 & 47 CAGE & ALAT1 & \\
\hline DRR176400 & CNhi10537_GCT. & T.experiment & argated & NA & 6RP11-422J8.1 & Homo sapiens & FANTOM6 project & PRJDB7993 & 17333569 & 47 CAGE & ASO_G0233621_00 & HDF (neonat \\
\hline & CNhii & experiment & $\begin{array}{l}\begin{array}{l}\text { Experiment } \\
\text { control }\end{array} \\
\text { ant }\end{array}$ & $\mathrm{NA}$ & 6/MALAT1 & Homo sapiens & project & & 9629498 & & ASO_MALAT1 & \\
\hline & & & Reference & NA & & Homo sapiens & 6 project & PRJD & 9434856 & & & \\
\hline & CNhii & j.experiment & Targeted & NA & 6AC0121466.7 & Homo sapiens & 6 project & PRJD & 13874137 & & 327. & HDF (ne \\
\hline 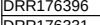 & & T.experiment & | Negative control & NA & $5 \mathrm{NA}$ & $\begin{array}{l}\text { Homo sapiens } \\
\text { Homs }\end{array}$ & $\frac{16 \text { project }}{6 \text { rect }}$ & $\frac{P R}{P D P}$ & 14349591995 & & $\frac{4}{4}$ & HDF (n) \\
\hline DRR & chlis & Te experiment & Targeted & $\frac{N A}{N A}$ & $1 \mathrm{P}$ & Homo sapiens & (1) poroect & & & & 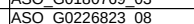 & HDF \\
\hline & & $\begin{array}{l}\text { G.experiment } \\
\end{array}$ & Fargeted & NA & $4 \mathrm{~N}$ & Homo sapiens & 6 project & & 13 & & $\begin{array}{ll}06 \\
06\end{array}$ & \\
\hline & & C.experi & argeted & $\mathrm{NA}$ & 4/RP5-1103G7.4 & Homo sapiens & 16 project & PRJD & & & 2537705 & HDF \\
\hline \begin{tabular}{|l|l|} 
DRR176327 & \multicolumn{1}{|c|}{} \\
\end{tabular} & CNhi1 & S.experiment & Fargeted & NA & 4 SUGTIP & Homo sapiens & FANTOM6 project & PRJDB7993 & 1549 & & 882303 & HDF \\
\hline \begin{tabular}{|l|l|l|l|l|l} 
DRR17326 \\
DRR176325
\end{tabular} & $\begin{array}{l}\text { CNhi110528_AGT. } \\
\text { CNhhi1528 ACG. }\end{array}$ & $\begin{array}{l}\text { T.experiment } \\
\text { Gexperiment }\end{array}$ & $\begin{array}{l}\text { Targeted } \\
\text { Neagive control }\end{array}$ & $\frac{N A}{N A}$ & $\begin{array}{l}4 R P 11-545 E 1 \\
5 N A\end{array}$ & \begin{tabular}{|l|} 
Homo sapiens \\
Homo saaiens
\end{tabular} & \begin{tabular}{|l|} 
FANTOM6 project \\
FAATOM6 project
\end{tabular} & \begin{tabular}{|l} 
PRJDB79993 \\
PRJDP7993
\end{tabular} & \begin{tabular}{|c|}
13621681 \\
12110679 \\
\end{tabular} & $\begin{array}{l}47 \text { CAGE } \\
47 \text { CAGE }\end{array}$ & \begin{tabular}{|l|} 
ASO_G0223478 \\
ASO NC A
\end{tabular} & \begin{tabular}{|l} 
HDF (nec \\
HDF nect
\end{tabular} \\
\hline
\end{tabular}


Table_S3 Meatadata for single cell analysis and FANTOM6 kmer research

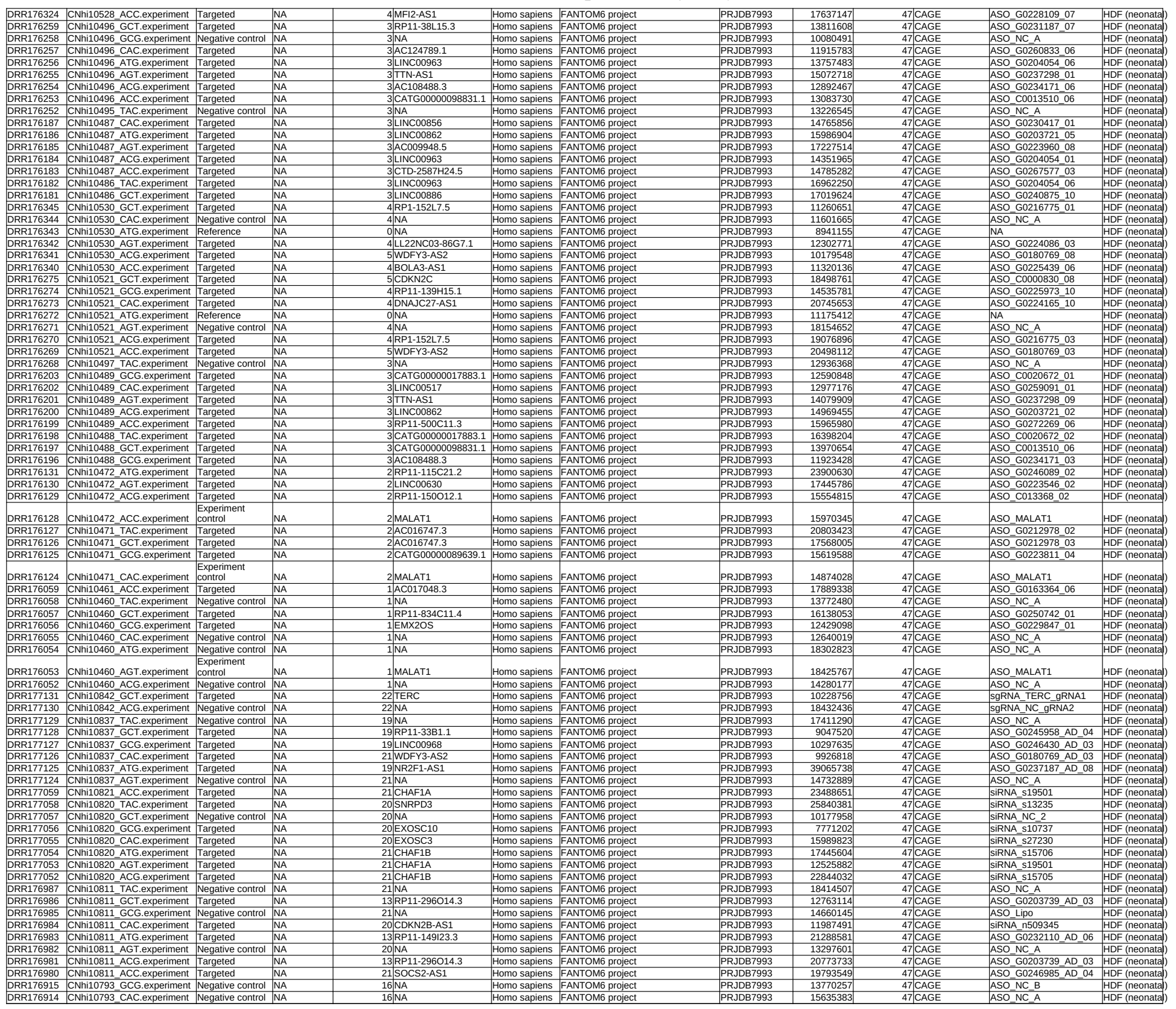


Table_S3 Meatadata for single cell analysis and FANTOM6 kmer research

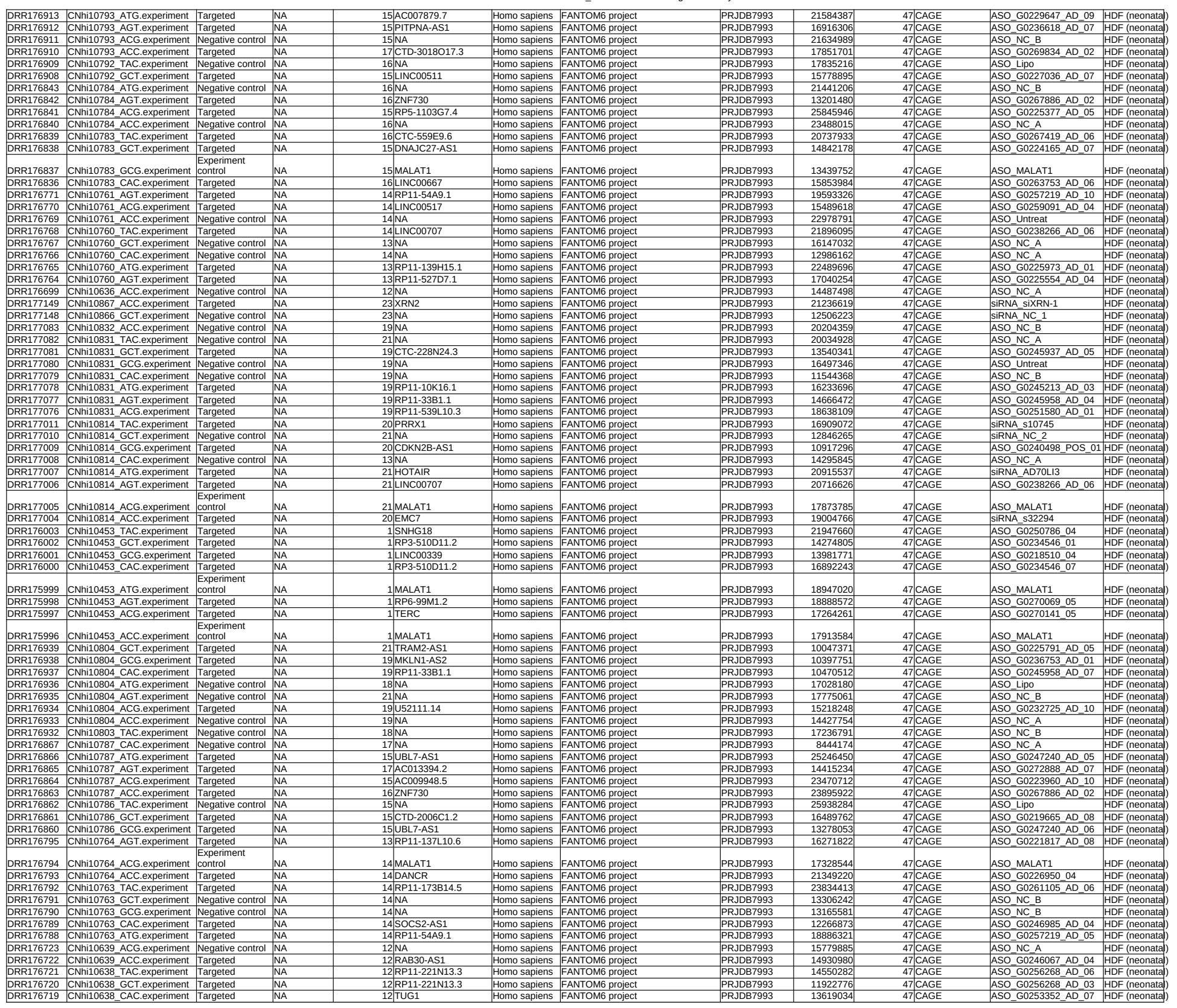


Table_S3 Meatadata for single cell analysis and FANTOM6 kmer research

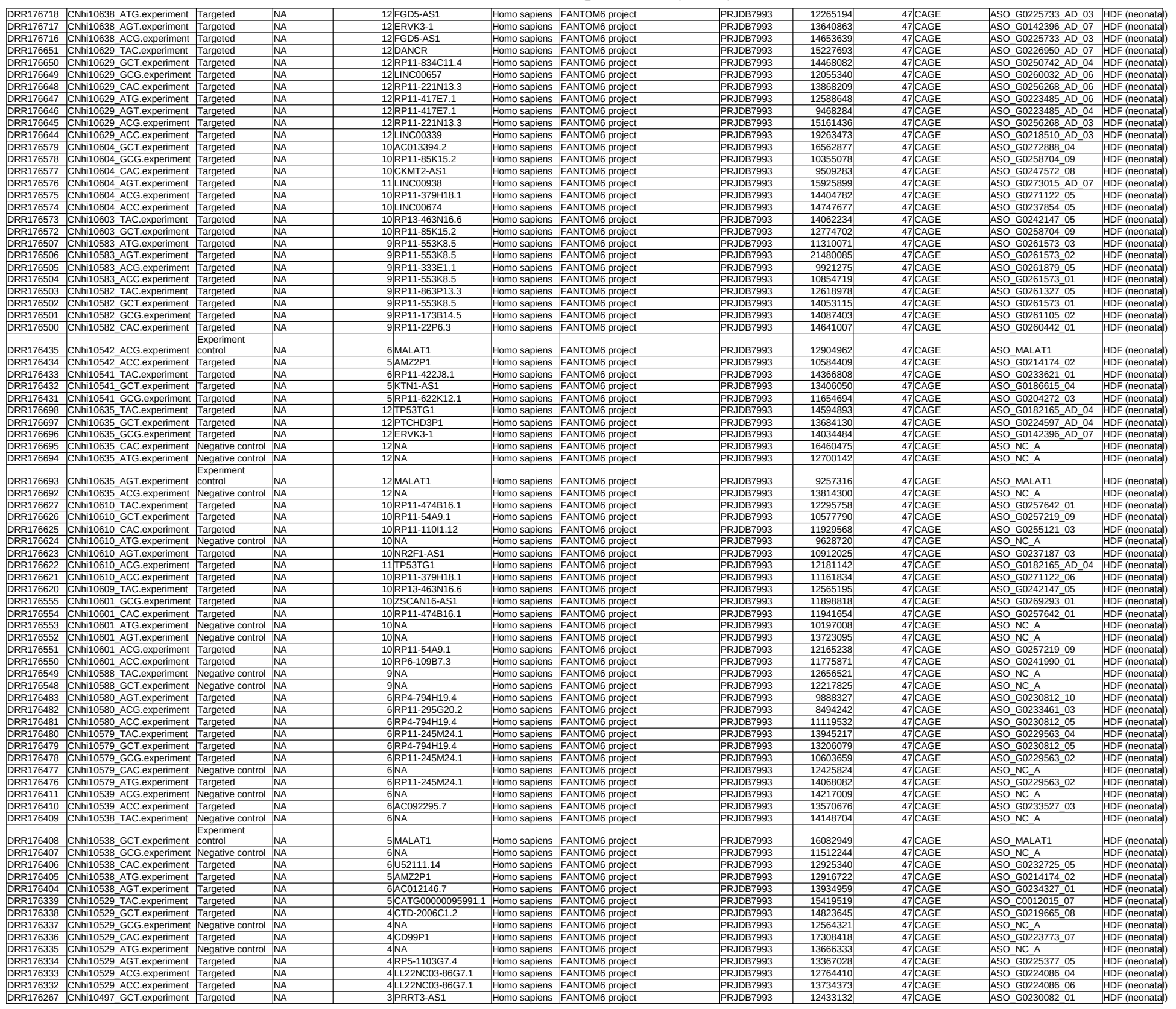

Page 17 
Table_S3 Meatadata for single cell analysis and FANTOM6 kmer research

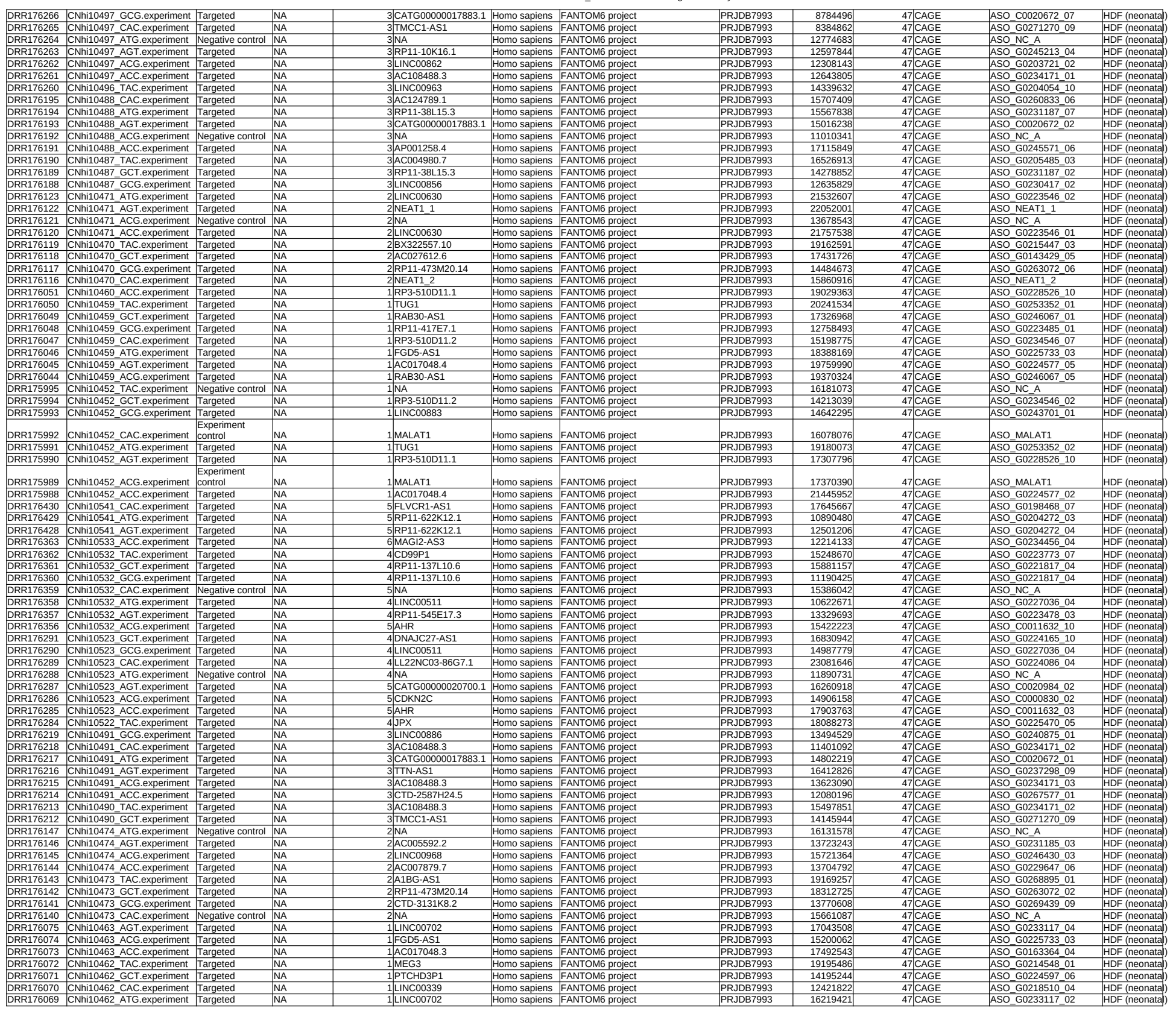


Table_S3 Meatadata for single cell analysis and FANTOM6 kmer research

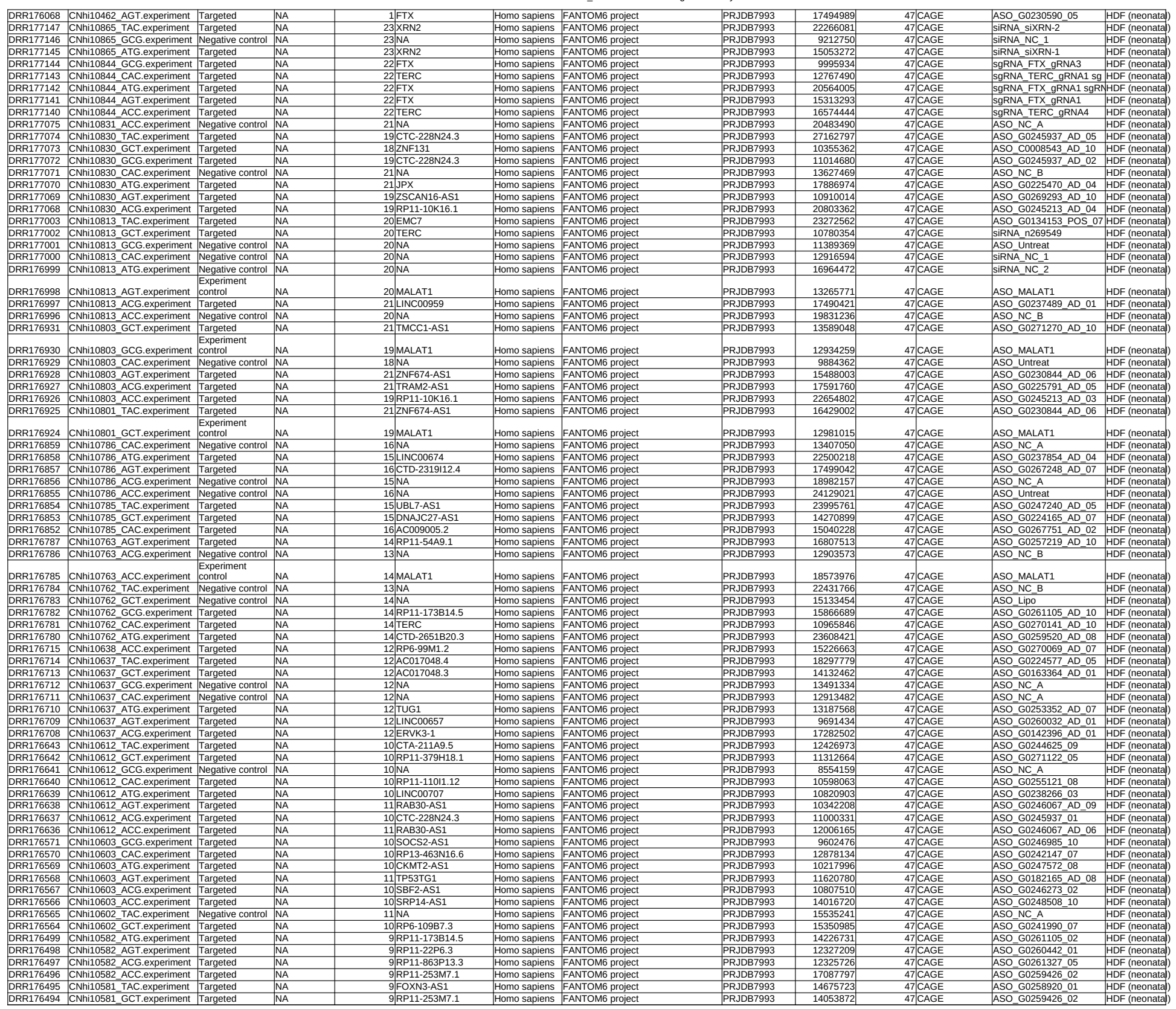


Table_S3 Meatadata for single cell analysis and FANTOM6 kmer research

\begin{tabular}{|c|c|c|c|c|c|c|c|c|c|c|c|}
\hline $\begin{array}{l}\text { DRR176493 } \\
\text { DR174692 }\end{array}$ & $\begin{array}{l}\text { CE.experiment } \\
\text { CC.experiment }\end{array}$ & $\frac{\text { Negative control }}{\text { Negative control }}$ & $\frac{N A}{N A}$ & $\frac{6 \text { NAA }}{6 \text { NA }}$ & $\begin{array}{l}\text { Homo sapiens } \\
\text { Homo sapiens }\end{array}$ & $\begin{array}{l}\text { FANTOM6 6 prjoect } \\
\text { FANTOM project }\end{array}$ & $\begin{array}{l}\text { PRJDD79993 } \\
\text { PRJDB7993 }\end{array}$ & $\mid \frac{12106554}{13966469}$ & 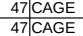 & $\frac{A S O \_N C A}{A S O N C \text { A } A}$ & $\begin{array}{l}\mathrm{HDF} \text { (neonata) } \\
\mathrm{HDF} \text { (neonatala }\end{array}$ \\
\hline & & & NA & \begin{tabular}{l|l|l|l|l|} 
RPP11-22011.1 \\
\end{tabular} & Homo sapiens & FANTOM6 project & PRJDB7993 & 11123665 & 47 CAGE & ASOGO20233137_05 & HDF (neonata) \\
\hline $\begin{array}{ll}\text { DRR176426 } \\
\end{array}$ & CNhi10541_ACC.experiment & Negative control & NA & $6 \mathrm{NA}$ & Homo sapiens & FANTOM6 project & PRJDB7993 & 11800868 & 47CAGE & ASO_NC_A & HDF (neonata) \\
\hline $\begin{array}{l}\text { DRR176425 } \\
\text { DR176424 }\end{array}$ & CNNhi10540_TAC.experiment & $\begin{array}{l}\text { Targeted } \\
\text { TTareted }\end{array}$ & $\frac{\mathrm{NA}}{\mathrm{NA}}$ & $\begin{array}{l}6 \text { 6/MAPKAPK5-AS1 } \\
\text { 5.CLDN22 }\end{array}$ & $\begin{array}{l}\text { Homo sapiens } \\
\text { Homo sapiens }\end{array}$ & $\begin{array}{l}\text { FANTOM6 project } \\
\text { FANTOM 6roject }\end{array}$ & $\begin{array}{l}\text { PRJDB79993 } \\
\text { PRJB7993 }\end{array}$ & \begin{tabular}{|l|}
11799170 \\
1398466 \\
\end{tabular} & $\begin{array}{l}47 \text { CAGE } \\
47 \text { CAGE }\end{array}$ & ASO_G0234608_06 & $\frac{\mathrm{HDF} \text { (neonatal) }}{\mathrm{HDF} \text { (neonatala }}$ \\
\hline $\begin{array}{l}\text { DRRR } \\
\text { DRR176424 }\end{array}$ & 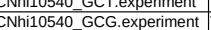 & $\begin{array}{l}\text { Larageted } \\
\text { Targeted }\end{array}$ & NA & $\begin{array}{l}\text { 5CLNOL2 } \\
\text { 5CATG00000016989.1 }\end{array}$ & $\begin{array}{l}\text { Homo saplens } \\
\text { Homo saiens }\end{array}$ & $\begin{array}{l}\text { FANIMB p prject } \\
\text { FANTOM6 project }\end{array}$ & $\begin{array}{l}\text { PRJUB } \\
\text { PRJDB7993 }\end{array}$ & \begin{tabular}{|l|}
13884666 \\
1104412 \\
\end{tabular} & $\begin{array}{l}4 \pi \text { CAGE } \\
477 \text { CAGE }\end{array}$ & 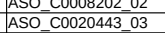 & 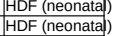 \\
\hline & CNhi10540 CAC.experiment & & NA & 5 RP11-622K12.1 & & FANTOM6 project & PRJDB7993 & $\begin{array}{ll}1100444147 \\
108447\end{array}$ & 477CAGE & SO_G0204272_04 & $\begin{array}{l}\text { HDF (neonatä) } \\
\text { HDF (neonatas) }\end{array}$ \\
\hline & CNhilos40_ATG.experiment & Targeted & NA & 5 CLDN22 & Homo sapiens & FANTOM6 project & PRJDB7993 & 12571318 & 47 CAGE & SO_CO008202_01 & HDF (neonatala) \\
\hline \begin{tabular}{l|l|l|l|l} 
DRR176420 \\
\end{tabular} & CNhi10540_AGT.experiment & Targeted & NA & 5 KTN1-AS1 & Homo sapiens & FANTOM6 project & PRJDB7993 & 13238245 & 477CAGE & ASO_60186615_09 & HDF (neonata) \\
\hline $\begin{array}{l}\text { DRR176355 } \\
\text { DR176354 }\end{array}$ & CNhi1 0532_A_C.experiment & $\begin{array}{l}\text { Targeted } \\
\text { Trancted }\end{array}$ & NA & $\begin{array}{l}\text { 4RP11-395B7.4 } \\
\text { ASUGTP }\end{array}$ & $\begin{array}{l}\text { Homo sapiens } \\
\text { Homp saiens. }\end{array}$ & FANTOM6 project & $\begin{array}{l}\text { PRJDB79933 } \\
\end{array}$ & 13374543 & 477CAGE & SO_G0227053_04 & HDF (neon \\
\hline & CNhni1i10531_TAC. & $\mid$ & $\begin{array}{l}\text { NA } \\
\text { NA }\end{array}$ & $\begin{array}{l}\frac{4 \text { SUGTIP }}{5 \text { NA }} \\
\end{array}$ & $\begin{array}{l}\text { Homo sapiens } \\
\text { Homo sapiens }\end{array}$ & $\begin{array}{l}\text { FANTOM6 project } \\
\text { FANTOMG b poject }\end{array}$ & $\begin{array}{l}\text { PRJJD79993 } \\
\text { PRJDB7993 }\end{array}$ & \begin{tabular}{|l|}
120157565 \\
1182056 \\
\end{tabular} & $\begin{array}{l}\text { 477CAGE } \\
47 \text { CAGE }\end{array}$ & $\begin{array}{l}\text { ASO_00226823_03 } \\
\text { ASO NC A }\end{array}$ & 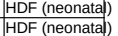 \\
\hline 3352 & 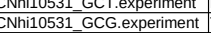 & Targeted & NA & $\frac{\text { 5/NA }}{5 \text { WDFY3-AS2 }}$ & $\begin{array}{l}\text { Homo saplens } \\
\text { Homo sapiens }\end{array}$ & $\begin{array}{l}\text { FANIOM } 6 \text { rjoect } \\
\text { FANTOM6 project }\end{array}$ & $\begin{array}{l}\text { PRJJER } \\
\text { PRJDB7993 }\end{array}$ & $\begin{array}{r}111802066 \\
8397642 \\
\end{array}$ & $\begin{array}{l}4 \pi \text { CAGE } \\
477 \text { CAGE }\end{array}$ & $\begin{array}{l}\text { ASO_NCA } \\
\text { ASO_G0180769_08 }\end{array}$ & 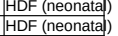 \\
\hline & & & $\mathrm{NA}$ & 5CATG00000020700.1 & & & PRJDB7993 & 11601366 & 477CAGE & & HDF (I \\
\hline DRP176350 & CNhilo531_ATG.experiment & Targeted & NA & 4RP4-773A18.4 & Homo sapiens & FANTOM6 project & PRJDB7993 & 10808666 & 47 CAGE & ASO_G0227811_06 & HDF (neona \\
\hline \begin{tabular}{|l|l} 
DRR176349 \\
\end{tabular} & CNhi10531_AGT.experiment & Targeted & NA & 4MFI2-AS1 & Homo sapiens & FANTOM6 project & PRJDB7993 & 11821692 & 47 CAGE & ASO_G0228109_07 & HDF (neon \\
\hline $\begin{array}{l}\text { DRR176348 } \\
\text { DRP176283 }\end{array}$ & CNNhi10531_AAG.experiment & Targeted & NA & $\begin{array}{l}\text { 4/RP11-395B7.4 } \\
\text { APCAT }\end{array}$ & Homo sapiens & FANTOM6 project & $\begin{array}{l}\text { PRJDB7993 } \\
\text { PRID7993 }\end{array}$ & $\begin{array}{l}1530324 \\
16904926 \\
\end{array}$ & $\begin{array}{l}47 \text { CAGE } \\
\text { A7CAGE }\end{array}$ & ASO_60227053_03 & HDF (neor \\
\hline & 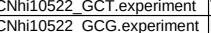 & Negated & $\begin{array}{l}\text { NAA } \\
\text { NA }\end{array}$ & $\begin{array}{l}4 \text { PINAT6 } \\
\text { 5NA }\end{array}$ & $\begin{array}{l}\text { Homo sapiens } \\
\text { Homo sapiens }\end{array}$ & $\begin{array}{l}\text { FANTOM6 6rjoett } \\
\text { FANTOM6 prjoect }\end{array}$ & $\begin{array}{l}\text { PRJJB79993 } \\
\text { PRJDB7993 }\end{array}$ & $\mid$\begin{tabular}{|l}
$\mid 16919426$ \\
13824230
\end{tabular} & $\begin{array}{l}\text { 477CAGE } \\
47 \text { CAGE }\end{array}$ & $\begin{array}{l}\text { ASO_G0228288_01 } \\
\text { ASO NC AA }\end{array}$ & $\begin{array}{l}\text { HDD (neonatat) } \\
\text { HDF (neoonata) }\end{array}$ \\
\hline $\begin{array}{l}\text { DRR176282 } \\
\text { DRR1728 }\end{array}$ & CNnhi1052_2_GAC.expereriment & Targeted & NA & $\frac{5 \mathrm{SNA}}{4 \mathrm{JPX}}$ & Homo sapiens & $\begin{array}{l}\text { FANIOM project } \\
\text { FANTOM6 project }\end{array}$ & 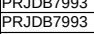 & 16348095 & $\begin{array}{l}47 C A C E \\
47 \text { CAGE }\end{array}$ & $\begin{array}{l}\text { ASO_NCA } \\
\text { ASO_G0225470 } 05\end{array}$ & $\begin{array}{l}\text { IDLF (neonatap) } \\
\mathrm{HDF} \text { (neonatat) }\end{array}$ \\
\hline & & & NA & \begin{tabular}{l|l|} 
CTTD-2006C1.2 \\
\end{tabular} & & & PRJDB7993 & 6267614 & 47 CAGE & ASO_G0219665_08 & HDF (neonata) \\
\hline DRR176279 & CNhilos22_AGT.experiment & Targeted & NA & 4CD99P1 & Homo sapiens & FANTOM6 project & PRJDB7993 & 18248582 & 47 CAGE & ASO_G0223773_05 & HDF (neonat \\
\hline 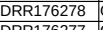 & CNhi10522_ACG.experiment & Targeted & NA & 4 CD27-AS1 & Homo sapiens & FANTOM6 project & PRJDB7993 & 15522154 & 47 CAGE & ASO_G0215039_01 & HDF (neor \\
\hline 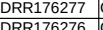 & CNhni10522_A_C.experiment & Targeted & NA & 5 AHR & Homo sapiens & FANTOM6 project & 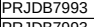 & 19880566] & $\begin{array}{l}47 \text { CAGE } \\
\text { A7CAGE }\end{array}$ & ASO_Co011632_03 & $\begin{array}{ll}\text { HDF (neo } \\
\text { HDF (neo }\end{array}$ \\
\hline DRR176211 / & CNhi1ino521_TAC.experiment & $\begin{array}{l}\text { Targeted } \\
\text { Targeted }\end{array}$ & $\frac{\text { NA }}{\mathrm{NA}}$ & 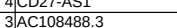 & $\begin{array}{l}\text { Homo sapiens } \\
\text { Homo sapiens }\end{array}$ & $\begin{array}{l}\text { FANTOM6 6 poject } \\
\text { FANTOM6 prjoett }\end{array}$ & $\begin{array}{l}\text { PRJDB/9933 } \\
\text { PRJDB7993 }\end{array}$ & $\begin{array}{l}183669999 \\
14460822\end{array}$ & $\begin{array}{l}\text { 477CAGE } \\
47 \text { CAGE }\end{array}$ & $\begin{array}{l}\text { ASO_GO2025039901 } \\
\text { ASO G0234171 } 06\end{array}$ & 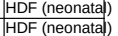 \\
\hline $\begin{array}{l}\text { DRR } \\
\text { DRR176210 }\end{array}$ & CNhi10490 CAC.experiment & $\begin{array}{l}\text { Taragelea } \\
\text { Targeted }\end{array}$ & NA & 3 LINCO08886 & $\begin{array}{l}\text { Homo saplens } \\
\text { Homo sapiens }\end{array}$ & & PRJDB7993 & 11307055 & 47CAGE & 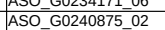 & $\begin{array}{l}\text { Hor neoun } \\
\text { HDF (neon }\end{array}$ \\
\hline & Experinem & & $\mathrm{NA}$ & 3 LINC00888 & Homo sapiens & FANTOM6 project & PRJDB7993 & 10000638 & 477CAGE & $A S O G C$ & $\mathrm{HDF}$ (neona \\
\hline DRR176208 & CNhilo490_AGT.experiment & Targeted & $\mathrm{NA}$ & 3/RP11-10K16.1 & Homo sapiens & FANTOM6 project & PRJDB7993 & 17275626 & 477CAGE & ASO_G0245213_04 & HDF (neon \\
\hline DRR176207 & CNhi10490_ACG.experiment & Targeted & NA & 3 LINC00856 & Homo sapiens & FANTOM6 project & PRJDB7993 & 13652351 & 47 CAGE & ASO_G0230417_01 & HDF (neo \\
\hline $\begin{array}{l}\text { DRR176206 } \\
\text { DRP176205 }\end{array}$ & experiment & $\begin{array}{l}\text { Targeted } \\
\text { Toroteds }\end{array}$ & NA & 3|LINC00517 & Homo sapiens & FANTOM6 project & $\begin{array}{l}\text { PRJDB79933 } \\
\text { DPID7093 }\end{array}$ & \begin{tabular}{|l|l|}
14077891 \\
171290
\end{tabular} & 47CAGE & ASO_G0259091_01 & HDF (n) \\
\hline $\begin{array}{l}\text { DRRR76205 } \\
\text { DRR176204 }\end{array}$ & 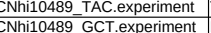 & $\mid$ & $\frac{N A}{N A}$ & $\begin{array}{l}\frac{3 R P 11-500 C 11.3}{3} \\
3 \text { NA }\end{array}$ & $\begin{array}{l}\text { Homo sapiens } \\
\text { Homo sapiens }\end{array}$ & $\begin{array}{l}\text { FANTOM6 project } \\
\text { FANTOM 6rjoett }\end{array}$ & $\begin{array}{l}\text { PRJJB79993 } \\
\text { PRJDB7993 }\end{array}$ & $\mid$\begin{tabular}{|l}
17142828 \\
15054983
\end{tabular} & $\begin{array}{l}\text { 477CAGE } \\
47 \text { CAGE }\end{array}$ & $\begin{array}{l}\text { ASO_C0272269_06 } \\
\text { ASO NC A }\end{array}$ & $\begin{array}{l}\text { HDF (neor } \\
\text { HDF neor }\end{array}$ \\
\hline $\begin{array}{l}\text { DRR } \\
\text { DRR176130 }\end{array}$ & $\begin{array}{l}\text { perment } \\
\text { periment }\end{array}$ & $\begin{array}{l}\text { Negative control } \\
\text { Targeted }\end{array}$ & NA & 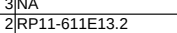 & $\begin{array}{l}\text { Homo sapiens } \\
\text { Homo sapiens }\end{array}$ & $\begin{array}{l}\text { FANTOM6 project } \\
\text { FANOF }\end{array}$ & $\begin{array}{l}\text { PRJDER9993 } \\
\text { PRIDB7993 }\end{array}$ & \begin{tabular}{|l|}
150549834 \\
19341728 \\
\end{tabular} & $\begin{array}{l}4 \pi \text { CAGE } \\
477 \mathrm{CAGE} \\
\end{array}$ & $\begin{array}{l}\text { ASO__NC_A } \\
\text { ASO G0257815 } 01\end{array}$ & \begin{tabular}{|l}
$\mathrm{HIF}$ (neona \\
$\mathrm{HDF}$ (neona
\end{tabular} \\
\hline 138 & eeriment & $\begin{array}{l}\text { Targeteu } \\
\text { Targeted }\end{array}$ & NA & $2 \mathrm{AC} 016747.3$ & Homo sapiens & TOM6 project & PRJDB7993 & 16812682 & 47 CAGE & ASO GO0212978_02 & HDF (neona \\
\hline DRR176137 & CNhilo473_ACG.experiment & Targeted & NA & $2 \mathrm{AC} 016747.3$ & Homo sapiens & FANTOM6 project & PRJDB7993 & 15343797 & 477CAGE & ASO_G0212978_04 & HDF (neo \\
\hline $\begin{array}{ll}\text { DRR176136 } \\
\text { DPD17025 }\end{array}$ & Nhni10473 ACC.experiment & Negative control & NA & 2 NA & Homo sapiens & FANTOM6 project & \begin{tabular}{|l} 
PRJDB79933 \\
DDP7003
\end{tabular} & \begin{tabular}{|l|l|}
19771257 \\
2318027
\end{tabular} & 47CAGE & ASO_NC_A & HDF (neor \\
\hline $\begin{array}{l}\text { DRRR7176135 } \\
\text { DRR176134 }\end{array}$ & experiment & $\begin{array}{l}\text { Targeted } \\
\text { Tarrageted }\end{array}$ & NA & $\frac{2 \mathrm{RP} 11-398 \mathrm{~K} 22.12}{2 \mathrm{RP} 1-1473 \mathrm{M} 2.14}$ & $\begin{array}{l}\text { Homo sapiens } \\
\text { Homo saniens }\end{array}$ & FANTOM6 project & PRJDB79993 & 23158002 & $\begin{array}{l}\text { 47CAGE } \\
47 \text { CAGEE }\end{array}$ & ASO_G0229852_05 & $\begin{array}{ll}\text { HDF (neen } \\
\text { HDE (neonen }\end{array}$ \\
\hline$\frac{D}{D R R}$ & & & NA & 2 ACO16747.3. & Homo sapiens & FANTOM6 project & $\begin{array}{l}\text { PRJDB } \\
\text { PRT993 }\end{array}$ & $\mid$ & $\begin{array}{l}\text { 47CAGE } \\
47 \text { CAGE }\end{array}$ & 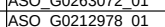 & $\frac{H D F(n)}{H D F(n}$ \\
\hline DRR176132 & CAC.experiment & Toractered & NA & 2RP11-473M20.14 & Homo sapiens & FANTOM6 project & PRJDB7993 & 15159810 & 47CAGE & ASO G02630720.05 & HDFF (n) \\
\hline DRR176067 & CNhi10462_ACG.experiment & Negative control & NA & $11_{\mathrm{NA}}$ & Homo sapiens & FANTOM6 project & PRJDB7993 & 14049470 & 477CAGE & ASO_NC_A & $\operatorname{HDF}(\mathrm{n})$ \\
\hline & CNhi10462_ACC.experiment & & NA & 1 1RP6-99M1.2 & Homo sapiens & FANTOM6 project & PRJDB7993 & 20499075 & 477CAGE & ASO $G 027006905$ & HDF (neon \\
\hline 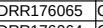 & periment & Targeted & NA & \begin{tabular}{l|l|l|l|} 
RP3-510D11.2 \\
\end{tabular} & Homo sapiens & FANTOM6 project & $\begin{array}{l}\text { PRJDB79933 } \\
\text { DDD7002 }\end{array}$ & $\begin{array}{ll}17807326 \\
\end{array}$ & 477CAGE & ASO_G0234546_01 & HDF (I) \\
\hline DRR176063 & $\begin{array}{l}\text { GCT.experiment } \\
\text { CAC experiment }\end{array}$ & larageted & NA & $\begin{array}{l}1 \text { DANCR } \\
1 \text { 1EMX2OS }\end{array}$ & $\begin{array}{l}\text { Homo sapiens } \\
\text { Homo saniens }\end{array}$ & $\begin{array}{l}\text { FANTOM6 project } \\
\text { FANTOMG proiect. }\end{array}$ & $\begin{array}{l}\text { PRJDB79993 } \\
\text { PRJBP7993 }\end{array}$ & \begin{tabular}{|r|r|}
17579848 \\
12102928
\end{tabular} & $\begin{array}{l}47 \text { CAGE } \\
47 \text { CAGE }\end{array}$ & & $\begin{array}{ll}\text { HDF (neo } \\
\text { HDF (neo }\end{array}$ \\
\hline $\begin{array}{ll}\text { DRR16063 C } \\
\text { DRR176062 }\end{array}$ & $\begin{array}{l}\text { SAC.experiment } \\
\text { TG experiment }\end{array}$ & Talageted & NAA & 1DACR & $\begin{array}{l}\text { Homo saplens } \\
\text { Homo saniens }\end{array}$ & $\begin{array}{l}\text { FANTOM6 p project } \\
\text { FANTOM proect }\end{array}$ & PRIDP793 & $\frac{133893938}{15651015}$ & $\begin{array}{l}47 \mathrm{CACE} \\
7 \mathrm{CAGE}\end{array}$ & 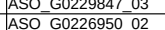 & $\begin{array}{l}\text { HDF (neen } \\
\text { HDF (neon }\end{array}$ \\
\hline 661 & CNhi1040461 & $\begin{array}{l}\text { Talageleu } \\
\text { Targeted }\end{array}$ & $\mathrm{NA}$ & 1RAB30-AS1 & $\begin{array}{l}\text { Homo sapiens } \\
\text { Homs sains }\end{array}$ & FANTOM6 project & PRJDB7993 & 21575839 & 47CAGE & 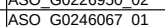 & HDF (neon \\
\hline & CNhi- & & $\mathrm{NA}$ & & Homo sapiens & $\frac{10 \text { project }}{16}$ & 93 & 13530144 & & SO NC A & HDF (I \\
\hline DRR177139 & c.experiment & Negative control & $\mathrm{NA}$ & 22 22A & Homo sapiens & FANTOM6 project & PRJDB7993 & 23443023 & 47 CAGE & SgRNA_NC_GRNA1 & HDF (neo \\
\hline $\begin{array}{l}\text { DRR177138 } \\
\text { DPR177127 }\end{array}$ & xperiment & Targeted & NA & 22 TERC & Homo sapiens & TOM6 project & \begin{tabular}{|l} 
PRJDB7993 \\
\end{tabular} & 12180047 & 47.CAGE & SgRNA_TERC_GRNA1 & HDF (neo \\
\hline 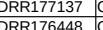 & xperiment & $\begin{array}{l}\text { Targeted } \\
\text { TTareted }\end{array}$ & NA & $\begin{array}{l}22 \mid F T X \\
5 C D N 22\end{array}$ & $\begin{array}{l}\text { Homo sapiens } \\
\text { Homo saniens }\end{array}$ & FANTOM6 project & 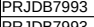 & \begin{tabular}{|l|l|}
12824896 \\
14503377
\end{tabular} & $\begin{array}{l}\text { 47CAGE } \\
47 \text { CAGE }\end{array}$ & $\begin{array}{l}\text { SgRNA_FTX__RRNA3 } \\
\text { ASO C0008202 } 01\end{array}$ & $\begin{array}{ll}\text { HDF (nee } \\
H D F(n e o\end{array}$ \\
\hline $\begin{array}{l}\text { DRR16448 } \\
\text { DRR176447 }\end{array}$ & $\begin{array}{l}\text { ppermiment } \\
\text { periment }\end{array}$ & $\begin{array}{l}\text { Iargeted } \\
\text { Negative control }\end{array}$ & $\begin{array}{l}\text { NA } \\
\text { NA }\end{array}$ & $\frac{56 \text { LEN22 }}{6 \text { NA }}$ & $\begin{array}{l}\text { Homo saplens } \\
\text { Homo sapiens }\end{array}$ & 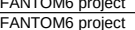 & $\begin{array}{l}\text { PRJUB/9993 } \\
\text { PRJDB7993 }\end{array}$ & $\begin{array}{l}145033217 \\
14323258\end{array}$ & $\begin{array}{l}\text { 47CAGE } \\
47 \text { CAGE }\end{array}$ & $\begin{array}{l}\text { ASO_CO0080202_01 } \\
\text { ASO NC A }\end{array}$ & $\begin{array}{l}\frac{H D F \text { (neo }}{H D F(n e o} \\
\mathrm{DF}\end{array}$ \\
\hline DRR176446 & CNhi10543 GCG.experiment & Targeted & NAA & 6/MAPKAPK5-A & 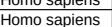 & FANTOM6 project & PRJDB7993 & $\begin{array}{l}1450200 \\
11940335 \\
\end{array}$ & $\begin{array}{l}47 C A C E \\
47 \text { CAGE }\end{array}$ & ASO_GOA & HDF (neone \\
\hline & iment & Targeted & NA & 6 ZNF37BP & Homo sapiens & ns project & PRJDB7993 & $\mid 11394708$ & & $34420=01$ & HDF ( \\
\hline DRR176444 C & CNhi10543_ATG.experiment & Targeted & NA & 5 CATG00000090305.1 & Homo sapiens & FANTOM6 project & PRJDB7993 & 12174880 & 47) CAGE & $11140=06$ & HDF (nec \\
\hline & experiment & $\begin{array}{l}\text { Targeted } \\
\text { Toted }\end{array}$ & NA & 6 AC092205.7 & Homo sapiens & TTOM6 project & $\begin{array}{l}\text { PRJDB7993 } \\
\end{array}$ & & & & HDF (neo \\
\hline DRR176378 & CNhi10534_TAC.experiment & Targeted & $\mathrm{NA}$ & 5/AMZ2P1 & Homo sapiens & FANTOM6 project & PRJDB7993 & 12328135 & 477CAGE & ASO_G0214174_06 & HDF (neon \\
\hline ORR1 & experiment & $\begin{array}{l}\text { Experimen } \\
\text { control } \\
\text { cons }\end{array}$ & NA & 5 MALAT1 & Homo sapiens & FANTOM6 project & PRJDB7993 & 15226344 & 47) CAGE & ALAT1 & HDF (r) \\
\hline & & & NA & 6 AC012146.7 & & ct & & 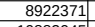 & tite & 27_07 & HDF (n) \\
\hline DRF & experiment & Targeted & NA & 5/AMZ2P1 & Homo sapiens & FANTOM6 project & PRJDB7993 & 10882945 & 477CAGE & 14177__06 & HDF $(n$ \\
\hline ic & experiment & Targeted & NA & 5 (KTI & Homo sapiens & project & & $\begin{array}{r}82142924] \\
1259527]\end{array}$ & $\begin{array}{l}\text { 47CAGE } \\
47 \text { CAGGE }\end{array}$ & $\begin{array}{l}04 \\
01\end{array}$ & \\
\hline & lexpermment & $\begin{array}{l}\text { Iargeted } \\
\text { Tareted }\end{array}$ & A & 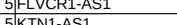 & $\begin{array}{l}\text { Homo saperens } \\
\text { Homo saing }\end{array}$ & $\begin{array}{l}6 \text { 6roject } \\
6 \text { 6ropect }\end{array}$ & 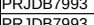 & $\begin{array}{l}12959527 \\
1126597\end{array}$ & $\begin{array}{l}\text { 47CACE } \\
7 \text { TCAGE }\end{array}$ & $\begin{array}{l}886801 \\
6615\end{array}$ & $\begin{array}{l}\frac{H D F(n}{H D F(n} \\
H D F\end{array}$ \\
\hline & experiment & Targeted & $\mathrm{NA}$ & 4 RP11 & Homo sapiens & project & 3 & 1744,25 & $477 \mathrm{C}$ & 02 & \\
\hline & tperiment & Targeted & $\mathrm{NA}$ & $5[R P 11$ & Homo sapiens & $c t$ & & & & 01 & \\
\hline & ent & Targeted & $\mathrm{NA}$ & 4LL22NC03-86G7.1 & Homo sapiens & $\frac{10 \text { project }}{16}$ & PRJDB7993 & 1417 & $47 / \mathrm{C}$ & 06 & HDF \\
\hline DRR & experiment & Targeted & $\mathrm{NA}$ & 5[RP11-474P2.4 & Homo sapiens & 16 project & PRJDB7993 & 16902977 & 477CAGE & $9316 \_08$ & HDF (nec \\
\hline & experiment & Targeted & NA & 5RP11-474P2.4 & Homo sapiens & 5 project & PRJL & 155565544 & 477CAGE & S_o8 & \\
\hline & 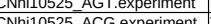 & Negalve conirol & NA & 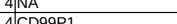 & $\begin{array}{l}\text { Homo Sapiens } \\
\text { Homo sains }\end{array}$ & $\begin{array}{lll}\text { poroject } \\
3\end{array}$ & 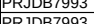 & $\frac{2153}{150}$ & 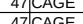 & so & $\begin{array}{l}\mathrm{HDF} \text { (neonatat) } \\
\mathrm{HDF} \text { (neonatat }\end{array}$ \\
\hline & St & Taraceted & NA & 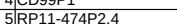 & Homo sapiens & th & & & 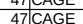 & & \\
\hline & experiment & Targeted & $\mathrm{NA}$ & $3 A \operatorname{Ac} 00$ & Homo sapiens & ct & & & & & HDF $(\mathrm{n}$ \\
\hline & experiment & Targeted & $\mathrm{NA}$ & 3 AC108488.3 & Homo sapiens & $6 \mathrm{prc}$ & PRJD & & & 99 & HDF (I \\
\hline c & experiment & Targeted & $\mathrm{NA}$ & $3 A C O$ & Homo sapiens & $5 \mathrm{prc}$ & PRJE & 1639 & $47 \mathrm{C} C \mathrm{C}$ & 02 & HDF \\
\hline & experiment & Targeted & NA & 32ZNF674-AS1 & Homo sapiens & $t$ & & 1495 & $47[\mathrm{C}$ & 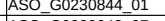 & HDF ( \\
\hline & expertment & largelea & NA & 3 & Homo saplens & 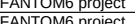 & 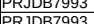 & $\frac{124}{121}$ & 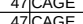 & & \\
\hline & & Targeted & $\mathrm{NA}$ & & Homo sapiens & $\mathrm{t}$ & & & & & \\
\hline 76228 & xperiment & Targated & $\mathrm{NA}$ & 3 3CTD-2 & Homo & FANTOM6 pro & PRJDB7993 & 6912842 & & 01 & 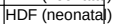 \\
\hline
\end{tabular}


Table_S3 Meatadata for single cell analysis and FANTOM6 kmer research

\begin{tabular}{|c|c|c|c|c|c|c|c|c|c|c|c|}
\hline $\begin{array}{l}\text { DRR176163 } \\
\text { DR176162 }\end{array}$ & 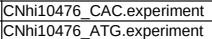 & $\begin{array}{l}\text { Targeted } \\
\text { Targated } \\
\end{array}$ & $\frac{N A}{N A}$ & $\begin{array}{l}\text { 2RP11-195F19.9 } \\
\text { 2RP11-65J3.1 }\end{array}$ & $\begin{array}{l}\text { Homo sapiens } \\
\text { Homo sapiens }\end{array}$ & \begin{tabular}{|l|} 
FANTOM6 project \\
FANTOM6 project
\end{tabular} & \begin{tabular}{|l} 
PRJDB7993 \\
PRJDB79933
\end{tabular} & \begin{tabular}{|l|l|}
14704089 \\
16087204 \\
\end{tabular} & $\begin{array}{l}47 \mathrm{CACAE} \\
47 \mathrm{CAGE}\end{array}$ & 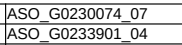 & $\begin{array}{l}\text { HDF (neonatat) } \\
\text { HDF (neonatat) }\end{array}$ \\
\hline $\begin{array}{l}\text { RR176161 } \\
\text { D17160 }\end{array}$ & CNhi10476_AGT.experiment & Targeted & $N A$ & $2 \mathrm{~B} \times 322557.10$ & Homo sapiens & FANTOM6 project & PRJDB7993 & 18493737 & $\begin{array}{l}47 \text { CAGE } \\
7 \text { CAGE }\end{array}$ & ASO_G02154470101 & HDF (neonate) \\
\hline & CNhi10476_ACG.experiment & & & 2A1BG-AS1 & Homo sapiens & & PRJDB7993 & 14631444 & $47 \mathrm{CAGE}$ & ASO_G0268895_03 & HDF (neonatat) \\
\hline 6159 & 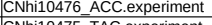 & argeted & NA & $\begin{array}{l}\text { 2|RP11-195F19.9 } \\
2 \text { RP1-65131 }\end{array}$ & Homo sapiens & FANTOM6 project & PRJDB7993 & 207368609 & 477CAGE & ASO_G0230074_07 & HDF (neonatat) \\
\hline $\begin{array}{l}\text { DRR176157 } \\
\text { DRR }\end{array}$ & 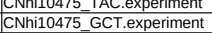 & $\begin{array}{l}\text { Ialgeied } \\
\text { Targeted }\end{array}$ & NA & 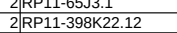 & $\begin{array}{l}\text { Homo Saplens } \\
\text { Homo sapiens }\end{array}$ & $\begin{array}{l}\text { FANIOM6 project } \\
\text { FANTOM6 project }\end{array}$ & $\begin{array}{l}\text { PRJDBC993 } \\
\text { PRJDB7993 }\end{array}$ & 2029360394 & $\begin{array}{l}4 \pi \text { CAAGE } \\
477 \mathrm{CAGE} \\
\end{array}$ & $\begin{array}{l}\text { ASO_GO233901__2 } \\
\text { ASO G022985203 }\end{array}$ & \\
\hline DRR176156 & CNhi10475 CAC.experiment & $\begin{array}{l}\begin{array}{l}\text { Experimen } \\
\text { control }\end{array} \\
\text { a }\end{array}$ & NA & & & ANTOM6 project & PRJDB7993 & 15105188 & 47 CAGE & & \\
\hline DRR176091 & CNhi10467_AGT.experiment & Targeted & NA & 2RP11-195F19.9 & Homo sapiens & ANTOM6 project & PRJDB7993 & & & & \\
\hline & G.experiment & & & $\begin{array}{l}\text { 2SERTAD4-AS1 } \\
\end{array}$ & Homo sapiens & =ANTOM6 brjoect & PRJDB7993 & 186475944 & CAGE & & $\frac{H D F \text { (neona }}{\mathrm{HDF} \text { (neona }}$ \\
\hline DRR176089 & CNhi10467_ACC.experiment & Targeted & NA & $2 \mid R P 11-458021.1$ & Homo sapiens & AANTOM6 project & PRJDB7993 & 17286834 & 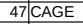 & 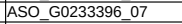 & \\
\hline & CNhi10466_TAC & & NA & & Homo sapiens & ANTOM6 project & & 24153957] & & & \\
\hline 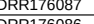 & CNhi110466_GCT.experiment & Targeted & $\frac{N A}{N A}$ & 2 AC027612.6 & Homo sapiens & 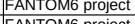 & $\mid$ & 166660899 & $\begin{array}{ll}47 \text { 47CAGE } \\
7 \text { CACEE }\end{array}$ & 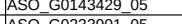 & HDF (neonat \\
\hline DR176085 & CNDhi1046- & 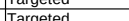 & & 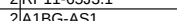 & Hom sapinins & FAATOM6 proiect & PRIDP093 & 1007010 & ATCAE & 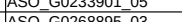 & HDF (neona \\
\hline & CNhi10466 ATG experinent & Tagrated & $\frac{N A}{N A}$ & 2 & & & PRJDB7993 & (184746677 & ATCAGE & 330607 & $\begin{array}{l}\text { HDF (neona } \\
\mathrm{HDF} \text { (neona }\end{array}$ \\
\hline DRR177091 & CNhi10833 ACC experient & | Negative control & $N A$ & $18 \mathrm{NA}$ & Homo sapiens & FANTOM6 project & PRJDB7993 & 1913487 7 & 47.CACE & ASO NC A & HDF (neopal \\
\hline DRR177090 & CNhilio832_TAC.experiment & Targeted & NA & 18CATG00000005991.1 & Homo sapiens & FANTOM6 project & PRJDB7993 & 19516719 & $47 \mathrm{CAGE}$ & ASO_C0012015_AD_02 & HDF (neona \\
\hline DRR177089 & CNhi10832_GCT.experiment & Negative control & NA & $199 \mathrm{NA}$ & Homo sapiens & FANTOM6 project & PRJDB7993 & 11832532 & 47 C7CAGE & ASO_Lipo & HDF (neo \\
\hline DR177088 & & Negative $c$ & NA & $19 \mathrm{NA}$ & Homo sapiens & FANTOM6 project & PRJDB7993 & 13300348 & 477CAGE & SO_NC_A & HDF (neona \\
\hline & & Tagrated & $\frac{N A}{N A}$ & 19.TDC. & & 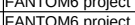 & (1) & 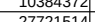 & AGE & O_G0226950_04 & HDF (neor \\
\hline & & & & 19 NR2F1-AS1 & & & & & & $\frac{A B O C}{A S O C}$ & $\begin{array}{l}\text { HDF (neoni } \\
\text { HDF (neopen }\end{array}$ \\
\hline DRR177084 & CNhiliogs32 ACG.evperiment & $\begin{array}{l}\text { Negative control } \\
\text { Negite }\end{array}$ & $N A$ & $19 \mathrm{NA}$ & Homo sapiens & FANTOM6 project & PRJDB7993 & 17993361 & 477CAGE & ASO NC B B & HDF (neona \\
\hline DRR177019 & CNhi10815_TAC.experiment & Targeted & NA & 20 20REC & Homo sapiens & FANTOM6 project & PRJDB7993 & 18537536] & 47 CAGE & 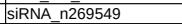 & HDF (neona \\
\hline DRR177018 & CNhi10815_GCT.experiment & Targeted & NA & 21 LINC00707 & Homo sapiens & FANTOM6 project & PRJDB7993 & 14406830 & 47 CAGEE & ASO_G0238266_AD_06 & HDF (neor \\
\hline DRR177017 & CNhi110815_GCG.experiment & Targeted & NA & $13 \mid 2 P 11-149123.3$ & Homo sapiens & FANTOM6 project & PRJDB7993 & 1325578783 & 477CAGE & ASO_G0232110_AD_06 & HDF (neont \\
\hline & 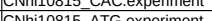 & & & & & EANTOM 6roject & & 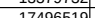 & ATCACE & $\begin{array}{l}\text { SIIINA ADAAYU4 } \\
\text { ASO NC A }\end{array}$ & HDF (neona \\
\hline$R R^{2} 1$ & CNhbi1085 & Targeted & & 20IMC- & & FANTOM6 proiect & PRIDP7993 & 17684100 & & ASO & $\begin{array}{l}\frac{H D F F \text { (neona }}{\mathrm{HDF} \text { (neona }} \\
\mathrm{H}\end{array}$ \\
\hline DRR177013 & 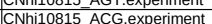 & $\begin{array}{l}\text { Talyetue } \\
\text { Targeted }\end{array}$ & $N A$ & 20EMC7 & Homo sapiens & FANTOM6 project & PRJDB7993 & 20573720 & 477CAGE & ASO G0134153 POS & HDF (ne \\
\hline DRR177012 & CNhi10815_ACC.experiment & Targeted & $\mathrm{NA}$ & 17|FOXN3-AS1 & Homo sapiens & FANTOM6 project & PRJDB7993 & 15320993 & 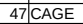 & ASO_G0258920 $A D$ & HDF (neo \\
\hline $\begin{array}{l}\text { DRR176011 } \\
\text { DR1700 }\end{array}$ & TAC.experiment & Targeted & NA & 11] 1 EMX20S & Homo sapiens & FANTOM6 project & 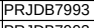 & 23299491 & 47 CAGE & ASO_G0229847_01 & HDF (neo \\
\hline DRR176010 & CNNhi10454_GCT.experiment & |Targeted & NA & 1PTCHO3P1 & Homo sapiens & FANTOM6 project & PRJDER9933 & 19931318 & 47/CAGE & ASOSOC0224597_05 & HDF (neona \\
\hline $\begin{array}{l}\text { DRR176009 } \\
\text { DRR176008 }\end{array}$ & $\begin{array}{l}\text { CNhn10454_GG.experment } \\
\text { CNnhi10454_CAC.experiment }\end{array}$ & \begin{tabular}{|l} 
Targeted \\
Negative control
\end{tabular} & NA & $1 \mathrm{NA}$ & $\begin{array}{l}\text { Homo sapients } \\
\text { Homo sains }\end{array}$ & FANTOM6 project & $\begin{array}{l}\text { PRJDB7993 } \\
\text { PRB }\end{array}$ & 129608323 & $\begin{array}{l}4 \pi \mathrm{CACE} \\
477 \mathrm{CAGE} \\
\end{array}$ & $\begin{array}{l}\text { ASO_062323485_O1 } \\
\text { ASO_NC_A }\end{array}$ & $\begin{array}{l}\text { HDF (neonatal) } \\
\text { HDF (neoonatat) }\end{array}$ \\
\hline DRR176007 & CNhi10454 ATG experiment & $\begin{array}{l}\text { Experiment } \\
\text { control }\end{array}$ & NA & 1 MALAT1 & Home saniens & =ANTOM 6 roiect & PRJPR7993 & 16224581 & A7 CAGE & ASO MATAT1 & \\
\hline & texperiment & Targated & NA & 1MEG3 & Homo sapiens & SM6 proiet & 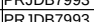 & 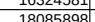 & & 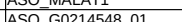 & \\
\hline DRR176005 & Gerpe & Targeted & NA & 1. PTCHD3P1 & Homo saniens & FATOM6 proiect & 79993 & 14512452 & & ASO & HDF \\
\hline DRR176004 & CNhi10454_ACC.experiment & Targeted & NA & 1AC0170048.4 & Homo sapiens & FANTOM6 project & PRJDB7993 & $\begin{array}{l}1412452 \\
1730792 \\
\end{array}$ & 477 CAGE & ASO_G0224577_01 & HDF ( \\
\hline DRR176947 & CNhi10805_GCT.experiment & Targeted & NA & 19 CTD-233602.1 & Homo sapiens & FANTOM6 project & PRJDB7993 & 12336277 & 47 CAGE & ASO_G0253982_AD_01 & HDF (ne \\
\hline DRR176946 & CNhi10805_GCG.experimiment & Targeted & $\mathrm{NA}$ & 1972SCAN16-AS1 & Homo sapiens & FANTOM6 project & PRJDB7993 & 10376190 & 477CAGE & ASO_G0269293_AD_10 & HDF (neo \\
\hline & 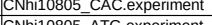 & Iragreted & NA & 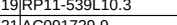 & Homo sapiens & $\begin{array}{l}\text { FANTOM6 project } \\
\text { FANT }\end{array}$ & $\mid$ & $\begin{array}{l}10497605 \\
10751\end{array}$ & & 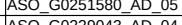 & HDF \\
\hline (N) & 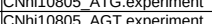 & $\begin{array}{l}\text { Targeted } \\
\text { Tarated }\end{array}$ & NAA & 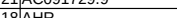 & Homo Saplents & 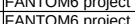 & P & $\frac{1759444}{1304707}$ & & A & $\begin{array}{ll}\text { HDF (ne } \\
H D D E(n e\end{array}$ \\
\hline DRR176943 & 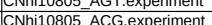 & $\begin{array}{l}\text { Tagreelea } \\
\text { Targeted } \\
\end{array}$ & NA & $19 \mathrm{RP} 1-33 \mathrm{~B} 11$ & Homo sapiens & FANTOM6 project & $\begin{array}{ll}P R J \\
P R J\end{array}$ & $\frac{1344407}{1646257}$ & & 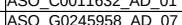 & $\begin{array}{ll}H D F(n \\
H D F(n\end{array}$ \\
\hline $\begin{array}{l}\text { DRR176942 } \\
\text { DRR176941 }\end{array}$ & CNni10805_ACC.experiment & Negative control & NA & $\frac{19}{18 \mathrm{NA}}$ & Homo sapiens & FANTOM6 project & PRJDB7993 & 16524020 & $\begin{array}{l}47 \mathrm{CAEE} \\
477 \mathrm{CAGE}\end{array}$ & ASO_Lipo & HDF (ne \\
\hline DRR176940 & CNhi10804_TAC.experiment & Negative control & NA & & Homo sapiens & FANTOM6 project & PRJDB7993 & 14869760 & 477CAGE & ASO_NC_A & HDF (nec \\
\hline DRR176875 & CNhi10788_CAC.experiment & Targeted & NA & 16 LINC00667 & Homo sapiens & FANTOM6 project & PRJDB7993 & 13995528 & 477CAGE & ASO_G0263753_AD_01 & \\
\hline DRR176874 & CNhi10788_ATG.experiment & $\begin{array}{l}\begin{array}{l}\text { Experime } \\
\text { control }\end{array} \\
\text { nats }\end{array}$ & NA & 16 MALAT1 & Homo sapiens & $=$ ANTOM 6 project & PRJDB7993 & 179858799 & 47 CAGE & Aso_t & HDF (ne \\
\hline DRR176872 & CNhi10788_AGT.experiment & $\begin{array}{l}\text { Targeted } \\
\text { Targated }\end{array}$ & NA & $\begin{array}{l}\text { 16/RP11-333E1.1 } \\
\text { 17AC013394. }\end{array}$ & Homo sapiens & $\begin{array}{l}\text { FANTOM6 project } \\
\text { FNTOM6 project }\end{array}$ & $\begin{array}{l}\text { PRJDB7993 } \\
\text { PRPBO }\end{array}$ & 15933723 & $\begin{array}{l}47 \text { 47CAGE } \\
47 \text { CAGE }\end{array}$ & ASO_G026 & \\
\hline (2) & 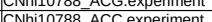 & 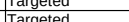 & NA & 17. & Hom spains & FANOMG no & (1) & 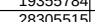 & & ASO- & \\
\hline DRR1 & CNhi1 ino & Targeted & NA & 16RP11-333E11 & Homo sapiens & 6 project & PRJDB7993 & 24399594 & 47 CAGE & ASO G026187 & \\
\hline DRR176869 & T) & | Negative control & NA & $16 \mathrm{NA}$ & Homo sapiens & FANTOM6 project & PRJDB7993 & 15790649 & 477CAGE & ASO NC A & HDF (ned \\
\hline DRR176868 & CNhi10787_GCG.experiment & Targeted & $\mathrm{NA}$ & 16. CTC-559E9.6 & Homo sapiens & FANTOM6 project & PRJDB7993 & 14310408 & 47 CAGE & ASO_G0267419_AD_04 & HDF (neonata \\
\hline DRR176803 & ST.experiment & $\begin{array}{l}\text { Experiment } \\
\text { control }\end{array}$ & NA & & & & 3 & & & & \\
\hline 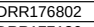 & CNhi10765_ACG.experiment & Targeted & NA & 14/RP11-553K8.5 & Homo sapiens & ANTOM6 project & PRJDB7993 & 1477 & $47 \mathrm{C}$ & ASO_G & \\
\hline & CAC.experiment & Targeted & NA & 22 TERC & Homo sapiens & FANTOM6 project & 7993 & 1363390309 & & $\begin{array}{l}\text { RC_gRNA1 } \\
\text { RCDPA1 }\end{array}$ & \\
\hline$\frac{1+n}{D R A}$ & Theneriment & $\begin{array}{l}\text { Tagrated } \\
\text { Tatas }\end{array}$ & NA & 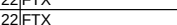 & Homo sapiens & 6 & & & & gRNA1 & \\
\hline & & Targated & NA & $22 \mathrm{~T}$ & Hor & ct & & & & NaAs & \\
\hline & 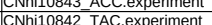 & Negative control & NA & $221 \mathrm{~N}$ & Homo saniens & 6 proiet & & 154488599 & & SgRNA NC GRNA1 & \\
\hline & Cexportivent & Ty & & 21RP11-398K22 12 & Homo sapiens & FANTOMG nreict & & & & & \\
\hline DE & & Negative control & NA & & Homo sapiens & th & 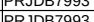 & 20557977 & & $\frac{5}{2}$ & \\
\hline & experiment & Tarceted & NA & $20 \mathrm{EXOSC3}$ & Homo: & t & & & & & \\
\hline DRR & Sexpeiment & $\begin{array}{l}\text { Tagrated } \\
\text { Tatas }\end{array}$ & NA & 20 SNRPD3 & Homo sapiens & 6rojoet & PR. & 10112718 & & & \\
\hline ORR1 & C.experiment & Targeted & $\mathrm{NA}$ & 20 EXOSC3 & Homo sapiens & 5 project & & & & & \\
\hline & & argeted & NA & & Homo sapiens & & & & & 234 & \\
\hline & experiment & Targeted & INA & 20 EXOSC10 & Homo sapiens & 6 project & & & & & \\
\hline & experiment & Fargeted & & 211CHAF1A & Homo sapiens & FAI & $\overrightarrow{P F}$ & & & SiRNA_ & \\
\hline & experiment & Targeted & $\mathrm{NA}$ & 2-AS1 & Homo sapiens & 5 pro & & & & $=01$ & \\
\hline & & Targrgeted & & 14SSOCS2-AS1 & Homo sapiens & project & & & & & \\
\hline & experim & legative control & NA & & Homo sapiens & 6 project & & & & ISO_U & \\
\hline & xperiment & Fargeted & INA & 21RPP11-527D7.1 & Homo sal & project & & 1396 & 477C & SO_C & \\
\hline & 12_ATG.experiment & eted & NA & 20|CDKN2B-AS1 & Homo sapiens & FANTOM6 project & $P R$ & 1649191942 & & 98 & \\
\hline & experiment & |largeled & 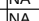 & 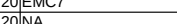 & $\begin{array}{l}\text { Homo Saplens } \\
\text { Hom sains }\end{array}$ & 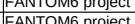 & 年 & & & & HUF \\
\hline & periment & $\begin{array}{l}\text { Tharated } \\
\text { Tarated }\end{array}$ & NA & 21HOTAIR & Homo saaiens & FANTM6 proiet & PRJDPG93 & 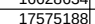 & (47CACE & SisNA & \\
\hline
\end{tabular}


Table_S3 Meatadata for single cell analysis and FANTOM6 kmer research

\begin{tabular}{|c|c|c|c|c|c|c|c|c|c|c|c|}
\hline DRR176923 & 11_GCG.ext & $\begin{array}{l}\begin{array}{l}\text { Experiment } \\
\text { control }\end{array} \\
\end{array}$ & NA & 21 MALAT1 & Homo sapiens & ANTOM6 project & PRJDB79993 & 10451398 & 477CAGE & & HDF (neon \\
\hline | DRR176922 & CNhi10801_CAC.experiment & \begin{tabular}{|l} 
Negative control \\
Tarcoted
\end{tabular} & NA & $\begin{array}{l}18 \text { NA } \\
18 \text { DANCF }\end{array}$ & Homo sapiens & $\begin{array}{l}\text { FANTOM6 } \text { project } \\
\text { FANTOG }\end{array}$ & $\begin{array}{l}\text { PRJDB7993 } \\
\end{array}$ & $\begin{array}{l}10331467 \\
1.57850909\end{array}$ & $47 \mathrm{CAGE}$ & ASO NC B B & HDF (neonate) \\
\hline $\mid$ & 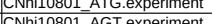 & $\begin{array}{l}\text { Targeted } \\
\text { Targeted }\end{array}$ & $\frac{N A}{N A}$ & $\begin{array}{l}18 \text { DANCR } \\
\text { 19NNT-AS1 }\end{array}$ & $\begin{array}{l}\text { Homo saplens } \\
\text { Hmong }\end{array}$ & $\begin{array}{l}\text { FANTOMG 6 project } \\
\text { FANTOM6 project }\end{array}$ & 年 & 151850598 & $\begin{array}{l}47 \mathrm{CAAE} \\
7 \mathrm{CACE}\end{array}$ & ASO_60226950 & HOF (neonatat) \\
\hline & 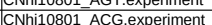 & 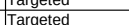 & NA & 18AHR & Hom spains & F & (1) & 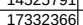 & $\begin{array}{l}4 \pi C A C E \\
47 C A G E\end{array}$ & 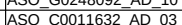 & $\begin{array}{l}\text { HDFF (neenatala) } \\
\text { HDF (neonatat) }\end{array}$ \\
\hline 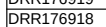 & CNhi10801 ACC.experiment & $\begin{array}{l}\text { Talyeteu } \\
\text { Targeted }\end{array}$ & NA & 18CATG00000095991.1. & Homo sapiens & FANTOM6 project & PRJDB7993 & $\begin{array}{ll}15325000 \\
1626024\end{array}$ & 477CAGE & ASO C0012015 AD 04 & HDF (neonatata) \\
\hline DRR176917 & CNhi10793_TAC.experiment & Targeted & NA & 15 ACO009948.5 & Homo sapiens & FANTOM6 project & PRJDB7993 & 17642958 & 47 CAGE & ASO_G0223960_AD_10 & HDF (neonat \\
\hline DRR176916 & CNhi10793_GCT.experiment & Targeted & NA & $\begin{array}{l}15 \text { RP5-1103G7.4 } \\
15 \text { CTA } 21190.5\end{array}$ & Homo sapiens & FANTOM6 project & PRJDB7993 & $\frac{16776427}{22301914}$ & $\begin{array}{ll}47 \mathrm{CAGE} \\
7 \mathrm{CAGE}\end{array}$ & ASO G0225377 ADDO- & HDF (neon) \\
\hline \begin{tabular}{|l|} 
RRR176851 \\
DRR176850
\end{tabular} & $\begin{array}{l}\text { CNhi10785_AAG.experiment } \\
\text { CNhi10785_AGT experiment }\end{array}$ & Targated & $\frac{N A}{N A}$ & $\frac{15 \text { [TTA-211A9.5 }}{16 \text { RP13-20L14.6 }}$ & $\begin{array}{l}\text { Homo sapiens } \\
\text { Homo sapiens }\end{array}$ & $\begin{array}{l}\text { FANTOM6 6rjoect } \\
\text { FANTOG project }\end{array}$ & $\mid$\begin{tabular}{|l|l|} 
PRJDBB9993 \\
PRJD7993
\end{tabular} & 234814144 & $\begin{array}{l}47 \mathrm{CAGE} \\
47 \mathrm{CAGE}\end{array}$ & 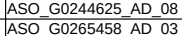 & $\frac{\mathrm{HDF} \text { (neonat }}{\mathrm{HDF} \text { (neonat }}$ \\
\hline & & & & & $\begin{array}{l}\text { Homo saplens } \\
\text { Homo sapiens }\end{array}$ & $\begin{array}{l}\text { FANTMOM } 6 \text { roject } \\
\text { FANTOM6 project }\end{array}$ & $\begin{array}{l}\text { PRJDBR9933 } \\
\text { PRJDB7993 }\end{array}$ & & & $\begin{array}{l}\text { ASO_G0265458_AD_03 } \\
\text { ASO Untreat }\end{array}$ & $\begin{array}{ll}\text { HDF (neonatat) } \\
\text { HDF (neonatat) }\end{array}$ \\
\hline 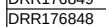 & CNhi10785 ACC.experiment & Targeted & $\mathrm{NA}$ & 17 17 CTD-3018017.3 & $\begin{array}{l}\text { Homo saplens } \\
\text { Homo sapiens }\end{array}$ & $\begin{array}{l}\text { FANIOMB project } \\
\text { FANTOM6 project }\end{array}$ & $\begin{array}{l}\text { PRJDBC993 } \\
\text { PRJDB7993 }\end{array}$ & $\frac{2365914}{1910270}$ & (47CACE & \begin{tabular}{|l|l|} 
ASO_Un_treat \\
ASO G69834_AD_02
\end{tabular} & $\begin{array}{l}\text { HDLF (neonatat) } \\
\text { HDF (neonatat) }\end{array}$ \\
\hline DRR176847 & CNhi10784_TAC.experiment & Targeted & $\mathrm{NA}$ & 15 (5TD-2006C1.2 & Homo sapiens & FANTOM6 project & PRJDB7993 & 20059309 & $\begin{array}{lll}47 \text { CAGE } \\
\end{array}$ & ASO_60219665 ${ }^{A D} 08$ & HDF (neonat \\
\hline & CNhi10784_GCT.experiment & Targeted & $N A$ & 15 PITPNA-AS1 & Homo sapiens & FANTOMG project & PRJDB7993 & 13142421 & 47 CAGE & ASO_G0236618_AD_-09 & HDF (neonat \\
\hline \begin{tabular}{|l} 
RRR1768845 \\
DRR176844
\end{tabular} & $\begin{array}{l}\text { CNhi10784_GC.experiment } \\
\text { CNhi10784_AC.experiment }\end{array}$ & $\begin{array}{l}\text { |legative control } \\
\text { Targated }\end{array}$ & $\frac{N A}{N A}$ & $\begin{array}{l}\frac{15 \text { NA }}{15 \text { UBL7-AS1 }} \\
\end{array}$ & $\begin{array}{l}\text { Homo sapiens } \\
\text { Homo sapiens }\end{array}$ & $\begin{array}{l}\text { IFNTOM 6 prjoct } \\
\text { FANTOO6 project }\end{array}$ & \begin{tabular}{|l|l|} 
PRJDDB9993 \\
PRJD7993
\end{tabular} & $\frac{14008049}{12253203}$ & $\begin{array}{l}47 \text { CAAGE } \\
47 \text { CAGE }\end{array}$ & $\begin{array}{l}\text { ASO_Untreat } \\
\text { ASO G0247240 AD 06 }\end{array}$ & 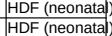 \\
\hline DRR176779 & CNhi10762 AGT experiment & $\begin{array}{l}\text { Experiment } \\
\text { control }\end{array}$ & NA & 17 MALAT1 & Homo sapiens & FANTOM6 project & PRJDB7993 & 8454 & 47 CAGE & & \\
\hline & CNhi10762_ACG.expe & Targeted & NA & 14RP5-886K2.3 & Homo sapiens & FANTOM6 project & PRJDB7993 & 117733373 & $\begin{array}{ll}47 \mathrm{CAC} \\
47 \mathrm{CAGE}\end{array}$ & ASO_G0236810_AD & HDF (neona \\
\hline DRR176777 & CNhi10762_ACC.experiment & Targeted & NA & 13 RP11-295G20.2 & Homo sapiens & FANTOM6 project & PRJDB7993 & 15374841 & $47 \mathrm{CAGE}$ & ASO_G0233461_AD_03 & HDF (neonatat) \\
\hline DRR176776 & CNhi10761_TAC.experiment & $\begin{array}{l}\text { Expermiment } \\
\text { control }\end{array}$ & NA & 13 MALAT1 & & FANTOM6 project & PRJDB7993 & 21544025 & 47 CAGE & & HDF (neonata) \\
\hline DRR176775 & CNhi10761_GCT.experiment & Targeted & NA & 13 RP11-149|23.3 & Homo sapiens & FANTOM6 project & PRJDB7993 & 123090900 & 47 CAGE & $\mathrm{ASO}=\mathrm{C}$ & HDF (neona \\
\hline 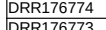 & CNhi10761_GCG.experiment & Targeted & NA & 13 DANCR & Homo sapiens & FANTOM6 project & $\begin{array}{l}\text { PRJDB7993 } \\
\text { PRPJPB93 }\end{array}$ & $\frac{12748501}{12501102}$ & 47 CAGE & ASO_G0226950_04 & HDF (neonatat) \\
\hline 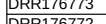 & CNhi110761__AC.experiment & $\begin{array}{l}\text { Negative control } \\
\text { Targeted }\end{array}$ & NA & $\begin{array}{l}14 \text { 14A } \\
14 \text { RAD51-AS1 }\end{array}$ & Homo sapiens & $\begin{array}{l}\text { FANTOM6 project } \\
\text { FANTOM6 nroect }\end{array}$ & $\begin{array}{l}\text { PRJDB7993 } \\
\text { PRPJP7993 }\end{array}$ & 125814992 & $\begin{array}{l}47 \text { CAGE } \\
47 \text { CAGE }\end{array}$ & ASO_NC_B & HDF (neonatat) \\
\hline DPR1672 & 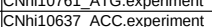 & $\begin{array}{l}\text { Targeled } \\
\text { Targeted }\end{array}$ & $\frac{N A}{N A}$ & 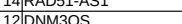 & 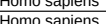 & 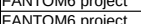 & $\begin{array}{l}\text { PRJDB7993 } \\
\text { PRPJP7993 }\end{array}$ & 245238890 & $\begin{array}{l}4 \pi C A E \\
47 C A G E\end{array}$ & 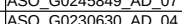 & 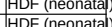 \\
\hline $\begin{array}{l}\text { DRR176706 } \\
\text { DRR }\end{array}$ & T-TAC.experiment & Tagrated & NA & TS & Homo sapiens & $\begin{array}{l}\text { FANIVIV project } \\
\text { FANTOM6 project }\end{array}$ & PRJDB7993 & $\begin{array}{l}16591381 \\
16918\end{array}$ & $\begin{array}{l}47 \mathrm{CACE} \\
47 \mathrm{CAE}\end{array}$ & 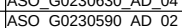 & $\begin{array}{l}\text { Hive (neonataf } \\
\text { HDF (neonatat) }\end{array}$ \\
\hline DRR176705 & CNhi10636_GCT.experiment & Targeted & NA & $12 A C 017048.3$ & Homo sapiens & FANTOM6 project & PRJDB7993 & 16509918 & 477CAGE & ASO_G0163364_AD_01 & HDF (neonate) \\
\hline DRR176704 & CNhi10636_GCG.experiment & Targeted & NA & 12 LINC00657 & Homo sapiens & FANTOM6 project & PRJDB7993 & 13636151 & 47 CAGE & ASO_G0260032_AD_06 & HDF (neonatat) \\
\hline \begin{tabular}{|l|} 
RRR176703 \\
DRR17602
\end{tabular} & CNhi10636_CAC.experiment & Targeted & NA & 12LLNC00657 & Homo sapiens & FANTOM6 project & $\begin{array}{l}\text { PRJDB7993 } \\
\text { PRPJD7993 }\end{array}$ & 13217433 & $\begin{array}{l}47 \text { 47AGE } \\
47 \text { CAE }\end{array}$ & ASO_G0260032_AD_01 & 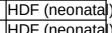 \\
\hline DRR176701 & CNhi10636 AGT.experiment & $\begin{array}{l}\text { Talgelea } \\
\text { Targeted } \\
\end{array}$ & $\mathrm{NA}$ & $\begin{array}{l}12 \mathrm{EM} X \mathrm{COS} \\
12 \mathrm{EM} 2 \mathrm{~S}\end{array}$ & $\begin{array}{l}\text { Homo Sapiens } \\
\text { Homo sapiens }\end{array}$ & FANTOM6 project & $\begin{array}{l}\text { PRJUDB9933 } \\
\text { PRJP7993 }\end{array}$ & $\begin{array}{ll}160484006 \\
11824543\end{array}$ & $47 \mathrm{CAGE}$ & 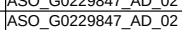 & $\begin{array}{l}\text { HDFF (neonatale) } \\
\text { HDF (neonatat) }\end{array}$ \\
\hline DRR176700 & CNhi10636_ACG.experiment & $\begin{array}{l}\text { Expernm } \\
\text { control }\end{array}$ & NA & 12 MALAT1 & Homo sapiens & FANTOM6 project & PRJDB7993 & 14694197 & 47) CAGE & ASO_MALAT1 & HDF (neonatal) \\
\hline DRR176635 & CNhi10611 TAC.experiment & \begin{tabular}{|l}
$\begin{array}{l}\text { Experiment } \\
\text { control }\end{array}$ \\
\end{tabular} & NA & 10|MALA & Homo sapiens & FANTOM6 project & PRJDB7993 & 566 & 477CAGE & ASO & HDF (neona \\
\hline DRR176634 & CNhi10611_GCT.experiment & Targeted & NA & 10/RP11-11011.12 & Homo sapiens & FANTOM6 project & PRJDB7993 & 8406979 & 47 CAGE & ASO_G0255121_08 & HDF (neona \\
\hline $\mid$ & CNhi10611_GCG.experiment & Negative control & NA & $\begin{array}{l}\text { 10]NA } \\
\end{array}$ & Homo sapiens & FANTOM6 project & PRJDB7993 & 9274218 & 477CAGE & ASO_NC_A & HDF (neonate) \\
\hline DRR116632 & E-CAC.experiment & IIargeted & $N_{A}$ & $10[R P 6-10987.3$ & Homo saplens & FANTMOM project & $\mid$ & 104693699 & 477CAGE & ASO_60241990_01 & HDF (neonatat) \\
\hline (1) & CNhH10611__ATGexperiment & 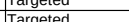 & NA & 10.2. & Hom spains & FANTOM Gropient & (1) & (125780 & $\begin{array}{l}4 \pi C A C E \\
47 C A G E\end{array}$ & ASO-60212888_04 & 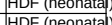 \\
\hline 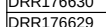 & CNhi10611__GGT.experiment & Tagretere & NA & 10 CTA-211AA9. 5 & Homo saniens & FANTOM6 project & PRJPR7993 & 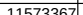 & 47 CAGE $>2$ ( & $A S O=0.024425=04$ & 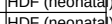 \\
\hline 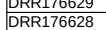 & 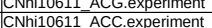 & $\begin{array}{l}\text { Ialgeted } \\
\text { Targeted }\end{array}$ & NA & 10 SOCS2-AS1 & $\begin{array}{l}\text { Homo saplens } \\
\text { Homo sapiens }\end{array}$ & $\begin{array}{l}\text { FANIOMG project } \\
\text { FANTOM6 project }\end{array}$ & PRJDB7993 & 109809067 & 477CAGE & ASO G0246985 & HDF (neonatata) \\
\hline DRR176563 & CNhi10602_GCG.experiment & Negative control & NA & 10 NA & Homo sapiens & FANTOM6 project & PRJDB7993 & 10065128 & 477CAGE & ASO_NC_A & HDF (neonatat) \\
\hline DRR176562 & CNhi10602_CAC.experiment & Negative control & NA & 10 NA & Homo sapiens & FANTOM6 project & PRJDB7993 & 10476544 & 477CAGE & ASO_NC_A & HDF (neonate) \\
\hline \begin{tabular}{|l|} 
DRR176561 \\
DRR176560
\end{tabular} & 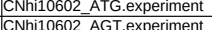 & \begin{tabular}{|l} 
Targeted \\
Neqative control
\end{tabular} & NA & 1.CTD-3018017.3 & $\begin{array}{l}\text { Homo sapiens } \\
\text { Homo sapiens }\end{array}$ & $\begin{array}{l}\text { FANTOM6 project } \\
\text { FANTOM6 project }\end{array}$ & $\begin{array}{l}\text { PRJDB7993 } \\
\text { PRPJD7993 }\end{array}$ & 120011933 & $\begin{array}{l}47 \text { CAGE } \\
47 \text { CAGE }\end{array}$ & $\begin{array}{l}\text { ASO_G0269834_02 } \\
\text { ASO NC A }\end{array}$ & 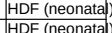 \\
\hline 年 & 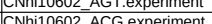 & Negatve control & NA & 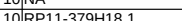 & $\begin{array}{l}\text { Homo Saplens } \\
\text { Hmo sains }\end{array}$ & 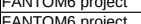 & PRIDP7993 & 10 & TICACE & $\mid$ & HDF (neonatat) \\
\hline 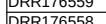 & 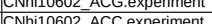 & 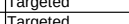 & NA & 10 & Homo sapiens & FANTOM Erient & PRIDB79093 & 12510693 & $A 7 C A C$ & ASO G0284508 04 & 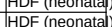 \\
\hline DRR176558 & $\begin{array}{l}\text { ACC.experiment } \\
\text { TAC.experiment }\end{array}$ & $\begin{array}{l}\text { Targeted } \\
\text { Targated } \\
\end{array}$ & $\frac{N A}{N A}$ & $\begin{array}{l}\text { 10SSRP4-AS1 } \\
\text { 11 LINC00883 }\end{array}$ & $\begin{array}{l}\text { Homo sapiens } \\
\text { Homo sapiens }\end{array}$ & $\begin{array}{l}\text { FANIVIV project } \\
\text { FANTOM6 project }\end{array}$ & $\begin{array}{l}\text { PRJUDBY93 } \\
\text { PRJDB993 }\end{array}$ & $\begin{array}{ll}12510109930 \\
17599940\end{array}$ & $\begin{array}{l}47 \mathrm{CAC} \\
47 \mathrm{CAE}\end{array}$ & ASO_G0243701_AD 08 & $\begin{array}{l}\text { HUIF (neonialat) } \\
\text { HDF (neonatat) }\end{array}$ \\
\hline DRR176556 & CNhi10601_GCT.experiment & Targeted & NA & 10 SRP14-AS1 & Homo sapiens & FANTOM6 project & PRJDB7993 & 13194337 & $47 \mathrm{CAGE}$ & ASO_G0248508_04 & HDF (neonatat) \\
\hline DRR1 & CNhii & Negative control & NA & 6 NA & Homo sapiens & FANTOM6 project & & 14020684 & 477CAGE & ASO_NC_A & HDF (neonate) \\
\hline \begin{tabular}{|l} 
DRR1764690 \\
DRR176489
\end{tabular} & $\begin{array}{l}\text { CNhi10588__ATT.experiment } \\
\text { CNhi10581_ACG.experiment }\end{array}$ & $\begin{array}{l}\text { Negative control } \\
\text { Targeted }\end{array}$ & $\frac{\mathrm{NA}}{\mathrm{NA}}$ & $\begin{array}{l}\text { 6NA } \\
\text { 9FOXN3-AS1 }\end{array}$ & $\begin{array}{l}\text { Homo sapiens } \\
\text { Homo sapiens }\end{array}$ & $\begin{array}{l}\text { FANTOM 6 project } \\
\text { FANTOM6 project }\end{array}$ & $\begin{array}{l}\text { PRJDD7993 } \\
\text { PRJDB7993 }\end{array}$ & $\begin{array}{ll}114030996 \\
10725955\end{array}$ & $\begin{array}{l}\text { 477CAGE } \\
477 \text { CAGE }\end{array}$ & $\begin{array}{l}\text { ASO_NCA } \\
\text { ASO G0258920 } 01\end{array}$ & $\begin{array}{l}\text { HDF (neonatat) } \\
\text { HDF (neonatal) }\end{array}$ \\
\hline DRR176488 & & \begin{tabular}{|l} 
Experiment \\
control
\end{tabular} & NA & & & EANTOM 6 roiect & & 12558472 & 47 CAGE & & ther (nentact \\
\hline & C.experiment & Targeted & NA & 9123.3 & Homo sapiens & project & $P R$ & 10467445 & $47 \mathrm{Cr}$ & ASO $G 023211004$ & \\
\hline DRR176486 & CNhi10580_GCT.experiment & Targeted & $\mathrm{NA}$ & $\begin{array}{l}6 \text { RP11-295G20.2 } \\
\end{array}$ & Homo sapiens & FANTOM6 project & & 10687172 & $47 \mathrm{C} / \mathrm{C}$ & ASO_G0233461_03 & \\
\hline & |CNhi1105050_GCG.experiment & Irargeted & $\mathrm{NA}$ & 6RP11-149123.3.3 & Homo sapiens & $\begin{array}{l}\text { FANTOMO project } \\
\text { FNTE }\end{array}$ & PRJDB7993 & 99002777 & 477CAGE & $\mid$ & \\
\hline DRR176484 & CNNhi10508_CAC.experiment & Ilargeted & NA & $6 \mid \mathrm{RP} 4-194 \mathrm{H} 19.4$ & Homo sapiens & FANIOML project & PRJDB/993 & 9966639 & $4 \pi$ 4/CAGE & ASO_G0230812_10 & HDF \\
\hline DRR1 & G.experiment & Negative control & NA & $6 / \mathrm{NA}$ & Homo sapiens & project & PFF & 10262820 & 477CAGE & ASO_NC_A & HDF (neone \\
\hline 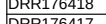 & ACC.experiment & Trargeted & NA & $\begin{array}{l}5 \text { CATG0000000016989.1 } \\
5\end{array}$ & Homo sapiens & 6 project & $\begin{array}{l}P R J \\
P R D\end{array}$ & 113273355 & $47 \mathrm{Cr}$ & 20443 206 & HDF (neon \\
\hline DRR1 & Texperiment & Tagrated & NA & $\frac{\mathrm{ACC}}{6 \mathrm{AC}}$ & Homo sapiens & FAN & $\mathrm{PE}$ & 158 & & ASO & $\begin{array}{lll}H D F \\
H D F\end{array}$ \\
\hline & 5.experiment & Targeted & NA & 50 & Homo sapiens & & & $106 \mathrm{C}-2 \mathrm{C}$ & & ASO & \\
\hline DRR: & C.experiment & Targeted & NA & 20090305.1 & Homo sapiens & FANTOM6 project & & 13275292 & $47 \mid C A C$ & ASO C0011140 06 & HDF (neona \\
\hline DRR176413 & CNhi10539_ATG.experiment & Targeted & NA & \begin{tabular}{|l|l|}
6 MAGI2-AS3 \\
\end{tabular} & Homo sapiens & FANTOM6 project & PRJDB7993 & 13121092 & 47 CAGE & ASO_G0234456_01 & HDF (neonate \\
\hline DRR1 & experiment & $\begin{array}{l}\text { Experment } \\
\text { control }\end{array}$ & NA & $5[\mathrm{MA}$ & Homo sapiens & FANT & PRJI & 1154 & 47) CAGE & & HDF \\
\hline DRR11 & experiment & Targeted & NA & $4 \mathrm{RP}$ & Homo sapiens & oject & & 117 & 477CAGE & ASO & HDF (ne) \\
\hline & & argeted & NA & & Homo sapiens & 6 project & PRJDB7993 & 32319 & 47 CAGE & & \\
\hline
\end{tabular}


Table_S4 metadata for kmer research in MSC in different conditions

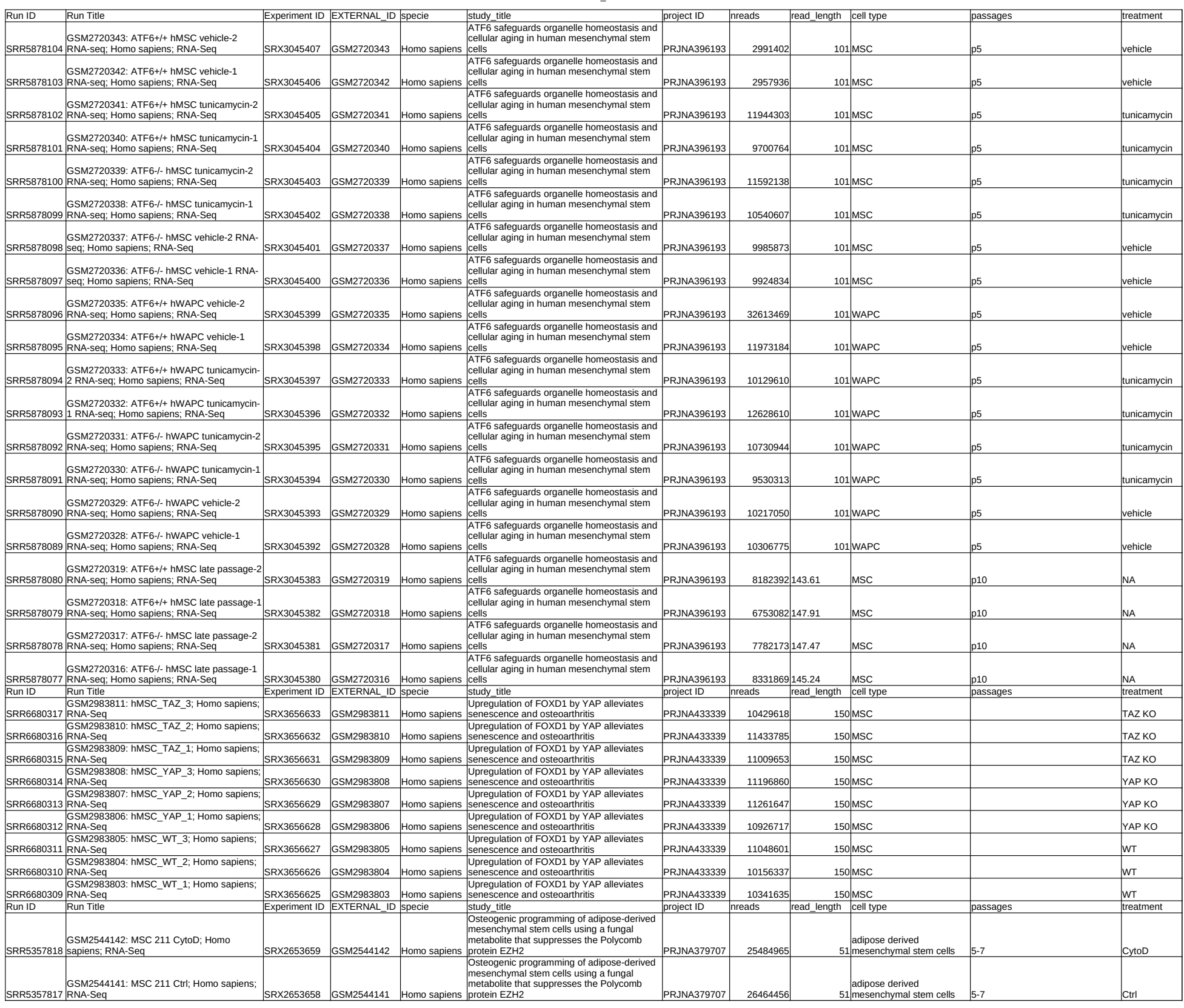


Table_S4 metadata for kmer research in MSC in different conditions

\begin{tabular}{|c|c|c|c|c|c|c|c|c|c|c|}
\hline SRR53578 & $\begin{array}{l}\text { GSM2544140: MSC } 283 \text { CytoD; Homo } \\
6 \text { sapiens; RNA-Seq }\end{array}$ & SRX2653657 & GSM2544140 & Homo sapiens & 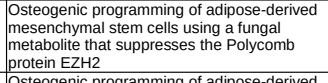 & PRJNA379707 & 24280737 & $\begin{array}{l}\text { adipose derived } \\
51 \text { mesenchymal stem cells } \\
\end{array}$ & $5-7$ & CytoD \\
\hline SRR5357815 & $\begin{array}{l}\text { GSM2544139: MSC } 283 \text { Ctrl; Homo sapiens; } \\
\text { SNA-Seq }\end{array}$ & SRX2653656 & GSM2544139 & Homo sapiens & $\begin{array}{l}\text { Sotteogenic programming of adidosese-derived } \\
\text { mesenchymmal stem cells using a tungal } \\
\text { metabolitet that suppresses the Polycomb } \\
\text { protein EZH2 }\end{array}$ & PRJNA379707 & 21040712 & $\begin{array}{l}\text { adipose derived } \\
51 \text { mesenchymal stem cells } \\
\end{array}$ & $5-7$ & ctrl \\
\hline SRR5357814 & $\begin{array}{l}\text { GSM2544138: MSC } 258 \text { CytoD; Homo } \\
\text { GSapiens; RNA-Seq }\end{array}$ & SRX2653655 & GSM2544138 & Homo sapiens & $\begin{array}{l}\text { Osteogenic programming of adipose-derived } \\
\text { mesenchymal stem cells usisg a tungalal } \\
\text { metabolite that suppresses the Polycomb } \\
\text { protein EZZ2 }\end{array}$ & PRJNA379707 & 35139902 & $\begin{array}{l}\text { adiposes derived } \\
51 \text { mesenchymmal stem cells }\end{array}$ & 5-7 & Cytoo \\
\hline SRR53578 & $\begin{array}{l}\text { GSM2544137: MSC } 258 \text { Ctrl; Homo sapiens; } \\
\text { RNA-Seq }\end{array}$ & SRX2653654 & GSM2544137 & Homo sapiens & $\begin{array}{l}\text { osteogenic programming of adipose-derived } \\
\text { mesenchymmal stem cells using a tungal } \\
\text { metabolite that suppresses the Polycomb } \\
\text { protein EZZ22 }\end{array}$ & PRJNA379707 & 34623656 & $\begin{array}{l}1 \text { adipose derived } \\
1 \text { mesenchymal stem cells }\end{array}$ & $5-7$ & $\mathrm{Ctrl}$ \\
\hline Run ID & Run Title & Experiment ID & EXTERNAL_ID & specie & 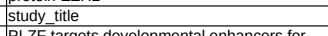 & project ID & nreads & read_length cell type & passages & treatment \\
\hline SRR8447838 & $\begin{array}{l}\text { GSM3564546: } 10 \text { days diff Control siRNA-C } \\
\text { 8RNA-seq; Homo sapiens; RNA-Seq }\end{array}$ & SRX5254873 & AMN1075560 & 3 Homo sapiens & 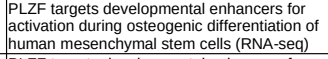 & PRJNA515466 & 17969174 & $\begin{array}{l}\text { human Mesenchymal Stem } \\
\text { 44Cells }\end{array}$ & p4-p10 & 10d os \\
\hline SRR84478 & $\begin{array}{l}\text { GSM3566545: } 10 \text { days diff Control siRNA-B } \\
\text { 7RAA-seq; Homo sapiens; } \text { RNA-Seq }\end{array}$ & SRX5254872 & SAMN1075560 & 4 Homo sapiens & 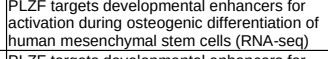 & PRJNA515466 & 18943085 & $\begin{array}{l}\text { human Mesenchymal Stem } \\
\text { 44 Cells }\end{array}$ & p4-p10 & 10d osteo Dif \\
\hline SRR8447 & $\begin{array}{l}\text { GSM3564544: } 10 \text { days diff Control siRNA-A } \\
6 \text { 6NA-seq; Homo sapiens; RNA-Seq }\end{array}$ & SRX5254871 & SAMN1075560 & 5 Homo sapiens & 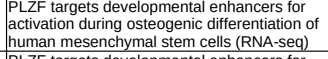 & PRJNA515466 & 26461685 & $\begin{array}{l}\text { human Mese } \\
\text { 44Cells }\end{array}$ & p4-p10 & 10d osteo Diff \\
\hline SRR844: & $\begin{array}{l}\text { GSM3564543: } 2 \text { days diff Control siRNA-C } \\
5 \text { RNA-seq; Homo sapiens; RNA-Seq }\end{array}$ & SRX5254870 & AMN1075: & Homo sapiens & 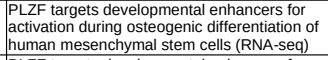 & PRJNA515466 & 27117845 & $\begin{array}{c}\text { human Mesenchymal Stem } \\
\text { 44Cells }\end{array}$ & p4-p10 & 2d osteo Diff \\
\hline SRR8447834 & $\begin{array}{l}\text { GSM3564542: } 2 \text { days diff Control siRNA-B } \\
4 \text { RNA-seq; Homo sapiens; RNA-Seq }\end{array}$ & SRX5254869 & SAMN1075560 & Homo sapiens & 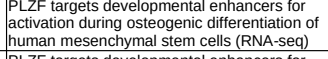 & PRJNA515466 & 26162294 & $\begin{array}{l}\text { 44 heman Mesenchymal Stem } \\
\text { Cens }\end{array}$ & p4-p10 & 2d osteo Diff \\
\hline SRR84 & $\begin{array}{l}\text { GSM3564541: } 2 \text { days diff Control siRNA-A } \\
\text { 3RNA-seq; Homo sapiens; RNA-Seq }\end{array}$ & SRX5254868 & SAMN1075560 & 8Homo sapiens & 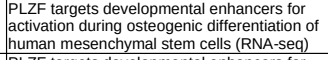 & PRJNA515466 & 19724867 & 44 Cells & $\mathrm{p4} 4 \mathrm{p} 10$ & 2d osteo Diff \\
\hline SRR84 & $\begin{array}{l}\text { GSM3564540: Naive Control siRNA-C RNA- } \\
\text { 2seq; Homo sapiens; RNA-Seq }\end{array}$ & SRX5254867 & MN1075 & Homo sapiens & 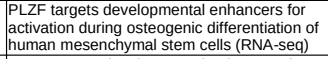 & PRJNA515466 & 266576 & $\begin{array}{c}\text { human } \\
\text { 44.Cells } \\
\text { Cons }\end{array}$ & p4-p10 & Naive \\
\hline SRR8447831 & $\begin{array}{l}\text { GSM3564539: Naive Control siRNA-B RNA- } \\
\text { Seq; Homo sapiens; RNA-Seq }\end{array}$ & SRX5254866 & SAMN1075561 & dHomo sapiens & 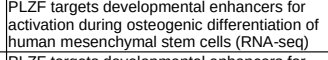 & PRJNA515466 & 25910627 & $\begin{array}{l}\text { human Mesench } \\
\text { 44 Cells }\end{array}$ & p4-p10 & Naive \\
\hline $\begin{array}{l}\text { SRR8447830 } \\
\text { Run ID }\end{array}$ & $\begin{array}{l}\text { GSM3564538: Nailve Control siRNA-A RNA- } \\
\text { oseq, Homm sapiens; RNA-Seq } \\
\text { Run Title }\end{array}$ & $\begin{array}{l}\text { SRX5254865 } \\
\text { Experiment ID }\end{array}$ & $\begin{array}{l}\text { SAMN1075561 } \\
\text { EXTERNALID }\end{array}$ & $\begin{array}{l}\text { Homo sapiens } \\
\text { specie }\end{array}$ & 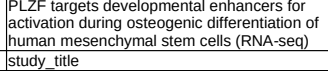 & \begin{tabular}{|l} 
PRJNA515466 \\
project ID
\end{tabular} & $\frac{24849706}{\text { eads }}$ & $\begin{array}{l}\text { 4human Mesenchymal Stem } \\
\text { 44 Cells } \\
\text { read_length cell type }\end{array}$ & $\begin{array}{l}\text { p4-p10 } \\
\text { passages }\end{array}$ & $\begin{array}{l}\text { Naive } \\
\text { treatment }\end{array}$ \\
\hline SRR3882937 & $\begin{array}{l}\text { GSM22314433: AMSC_19_proliferating; Homo } \\
7 \text { sapiens; RNA_Seq }\end{array}$ & SRX1939121 & GSM2231443 & Homo sapiens & $\begin{array}{l}\text { study) titet } \\
\text { Transtipe Sequencing of Adipose- } \\
\text { Derived Mesechymal Stromal Cells }\end{array}$ & PRJNA328824 & 29447967 & 50 & & \\
\hline SRR3882936 & $\begin{array}{l}\text { GSM2231442: AMSC_19_confluent; Homo } \\
\text { 6sapiens: RNA_Seq }\end{array}$ & SRX1939120 & GSM2231442 & Homo sapiens & $\begin{array}{l}\text { Transcriptome Sequencing of Adipose- } \\
\text { Derived Mesechymal stromal cells } \\
\end{array}$ & PRJNA328824 & 43754165 & $\begin{array}{l}\text { Adipose-derived } \\
51 \text { Mesenchymal Stromal Cell } \\
\end{array}$ & & \\
\hline SRR3882935 & $\begin{array}{l}\text { GSM2231441: AMSC_4_proliferating; Homo } \\
\text { 5asapiens: RNA Seq }\end{array}$ & SRX1939119 & GSM2231441 & Homo sapiens & $\begin{array}{l}\text { Transcriptome Sequencing of Adipose- } \\
\text { Derived Mesectymal Stromal Cells } \\
\end{array}$ & PRJNA328824 & 34428851 & 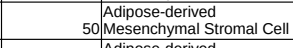 & & \\
\hline SRR3882934 & $\begin{array}{l}\text { 6SM2231440: AMSC_4_confluent; Homo } \\
4 \text { sapiens; RNA-Seq }\end{array}$ & SRX1939118 & GSM2231440 & Homo sapiens & $\begin{array}{l}\text { Transcriptome Sequencing of Adipose- } \\
\text { Derived Mesechymal Stromal Cells }\end{array}$ & PRJNA328824 & 42327600 & $\begin{array}{l}\text { Adipose-derived } \\
51 \text { Mesenchymal Stromal Cell }\end{array}$ & & \\
\hline \begin{tabular}{|l|l|} 
SRR3882933 \\
\end{tabular} & $\begin{array}{l}\text { GSM22313439: AMSC_3_prolifierating; Homo } \\
3 \text { sapiens; RNA-Seq _ }\end{array}$ & SRX1939117 & GSM2231439 & Homo sapiens & $\begin{array}{l}\text { Transcriptome Sequencing of Adipose- } \\
\text { Derived Mesechymal Stromal Cells }\end{array}$ & PRJNA328824 & 33767562 & $\begin{array}{l}\text { Adipose-derived } \\
51 \text { Mesenchymal Stromal Cell }\end{array}$ & & \\
\hline SRR3882932 & $\begin{array}{l}\text { GSM2231438: AMSC_3_confluent; Homo } \\
\text { 2 sapiens; RAA-Seq }\end{array}$ & SRX1939116 & GSM2231438 & Homo sapiens & $\begin{array}{l}\text { Transcriptome Sequencing of Adipose- } \\
\text { Derived Mesechymal Stromal Cells } \\
\end{array}$ & PRJNA328824 & 44801550 & $\begin{array}{l}\text { Adipose-derived } \\
\text { 51 Mesenchymal Stromal Cell } \\
\end{array}$ & & \\
\hline \begin{tabular}{|l|l|} 
SRR3882931 & \\
\end{tabular} & $\begin{array}{l}\text { 1) GSM22231437: : AMSC_1_proliferating; Homo } \\
\text { sapies; RNASeq }\end{array}$ & SRX1939115 & GSM2231437 & Homo sapiens & $\begin{array}{l}\text { Transcriptome Sequencing of Adipose- } \\
\text { Derived Mesechymal Stromal Cells }\end{array}$ & PRJNA328824 & 37501459 & $\begin{array}{l}\text { Adiposes-derived } \\
\text { 50|Mesenchymal Stromal Cell }\end{array}$ & & \\
\hline SRR3882930 & $\begin{array}{l}\text { GSM2231436: AMSC_1_confluent; Homo } \\
\text { osapiens; RAA-Seq }\end{array}$ & SRX1939114 & & sapiens & $\begin{array}{l}\text { Transcriptome Sequencicing of Adipipse- } \\
\text { Derived Mesechymmal Stromal Cells }\end{array}$ & PRJNA328824 & 34863048 & 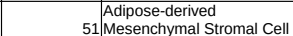 & & \\
\hline Run ID & Run Title & Experiment ID & EXTERNAL_ID & specie & study_title & project ID & nreads & read_length cell type & source_name & treatment \\
\hline SRR80 & $\begin{array}{l}\text { GSM3444217: EGF_IWR1_S03; Homo } \\
5 \text { sapiens; RNA-Seq }\end{array}$ & SRX4925800 & GSM3444217 & Homo sapiens & $\begin{array}{l}\text { Evidermal growtht factor activatest B-catenin } \\
\text { via integrin-linked kinasse to control } \\
\text { proliferation of mesenchymal stromal cells. }\end{array}$ & PRJNA498109 & 005865 & & & + IWR-1 \\
\hline SRR8099004 & $\begin{array}{l}\text { GSM3444216: EGF_IWR1_SO2; Homo } \\
4 \text { sapiens; RNA-Seq }\end{array}$ & SRX4925799 & GSM3444216 & Homo sapiens & $\begin{array}{l}\text { Epidermal grownth factor activatese } \beta \text { B-catenin } \\
\text { viantegrin-linked kinase to contro } \\
\text { proileratition of mesenchymal stromal cells. }\end{array}$ & PRJNA498109 & 11575790 & ienchymal stromal cells & femoral heads/knee bon & EGF + IWR-1 \\
\hline SRR8099 & $\begin{array}{l}\text { GSM3444215: EGF_IWR1_S01; Homo } \\
\text { 3. } \\
\text { sapiens; RNA-Seq }\end{array}$ & SRX4925798 & GSM3444215 & Homo sapiens & $\begin{array}{l}\text { EFidermal growth factoractivatese } \beta \text { B-catenin } \\
\text { via integrin-linked kinase to control } \\
\text { proliferation of mesenchymal stromal cells. }\end{array}$ & PRJNA498109 & 15666602 & enchymal stromal cells & femoral heads/knee bone explants & EGF + IWR- \\
\hline SRR80 & $\begin{array}{l}\text { GSM3444214: EGF_S03; Homo sapiens; } \\
\text { 2RNA-Seq }\end{array}$ & SRX4925797 & GSM3444214 & no sapiens & $\begin{array}{l}\text { Evidermal growth factor activatest } \beta \text { B-catenin } \\
\text { via integrin-linked kinase to conontro } \\
\text { proliferation of mesenchymal stromal cells. }\end{array}$ & PRJNA498109 & 15786160 & cells & dds/knee bone explants & EGF \\
\hline SRR8099001 & $\begin{array}{l}\text { GSM3444213: EGF_S02; Homo sapiens; } \\
1 \text { RNA-Seq }\end{array}$ & SRX4925796 & GSM3444213 & Homo sapiens & 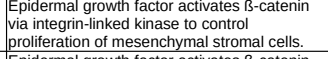 & PRJNA498109 & 19843529 & nchymals s. & femoral hea & EGF \\
\hline SRR80990 & $\begin{array}{l}\text { GSM3444212: EGF_S01; Homo sapiens; } \\
\text { orNA-Seq }\end{array}$ & SRX4925795 & GSM3444212 & Homo sapiens & $\begin{array}{l}\text { Exidermal grownth factor activatese B-catenin } \\
\text { via integrin-linked kinase to control } \\
\text { proliferation of mesenchymal stromal cells. }\end{array}$ & PRJNA498109 & 19132337 & nal cells & noral heads/knee bone explants & EGF \\
\hline SRR809 & $\begin{array}{l}\text { GSM3444211: untreated_S03; Homo sapiee } \\
\text { GNA-Seq }\end{array}$ & SRX4925794 & SSM3444211 & Homo sapiens & 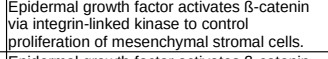 & PRJNA498109 & 13571062 & Mesenchymal stromal cells & femoral heads/knee bone explants & Intreated \\
\hline SRR8098998 & $\begin{array}{l}\text { GSM3444210: untreated_SO2; Homo sapiens; } \\
\text { 8RA-Seq }\end{array}$ & "SRX4925793 & GSM3444210 & Homo sapiens & $\begin{array}{l}\text { Epidermal grownth factortactivatese } \beta \text { B-catenin } \\
\text { via integrin-linked kinase to control } \\
\text { proilefration of mesenchymal stromal cells. }\end{array}$ & PRJNA498109 & 1667748 & & moral heads/knee bone explants & rea \\
\hline SRR8098997 & $\begin{array}{l}\text { 7) GSM3444209: untreated_S01; Homo sapi } \\
\text { RNA-Seq }\end{array}$ & SRX4925792 & GSM3444209 & Homo sapiens & $\begin{array}{l}\text { Exidermal growth factor activatese B-catenin } \\
\text { via integrin-linked kinase to control } \\
\text { proliferation of mesenchymal stromal cells. }\end{array}$ & PRJNA498109 & 17232973 & mal cells & noral heads/knee bone expla & untreated \\
\hline
\end{tabular}


FGF7
LRRC15

PTGFR
Alas5607.2

ALL355607.2
KCNK15
FGF5

SLFC1AA1

SMMLR
GDF5
GPS

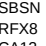

CA12
IL13RA2
EYA2

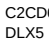

PENK
A.2359834.1

Als

297200.1
ACo22267

AC022467.

NTM

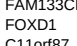

CI1ort87
ISR
SFRP4
HOXC10

PRR16

PRR16
HOXC11
ACC91112.2.
F

AM2258
ANC202454
IN

LAMA3

PDGRRA
LIMA1

LIMA1
ACO07336.3
BICCC1
old

OLFML1

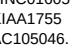

AC105046
ISFBP6
P

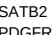

CLCA2

GPR1

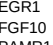

PAMP1
PANCO0968
PCOLCE
POLE

LINCO0068
PCoCE
POPDC

CIR
CCN5
CNS

COLAA1
SSPA

SSPN
APCDD1LDT
ACAP11

APCDD1L.D
AOCSS5
SOCS

Al355102.1

MOXD1

CLMP
GLYAL22

NTTN1
AC114284.1 


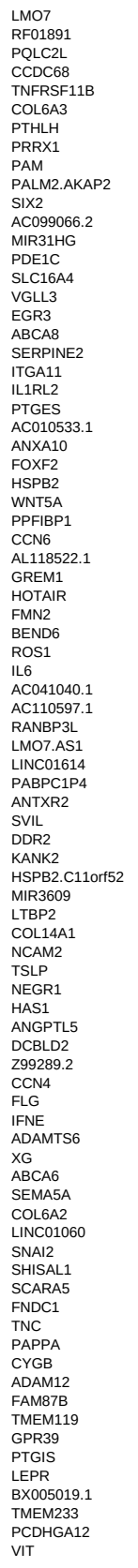

Table_S5 genes names positively enriched in MSC

PTHL

PALM2.AKAP2

MIR31HC

SLC16

ABCAB
SERPINE2

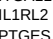

ANXA10

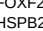

PPFiBP

ALL118522.1
GREM1

Ros1

AC1105972

INCOA1614

KAAN2 2 C.110r52

MiR3609

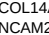

IFNE

LINCO1060

CYGB
CAMP

FAM87B

GPR39
PTGIS

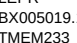
PCDHGA12
VIT 


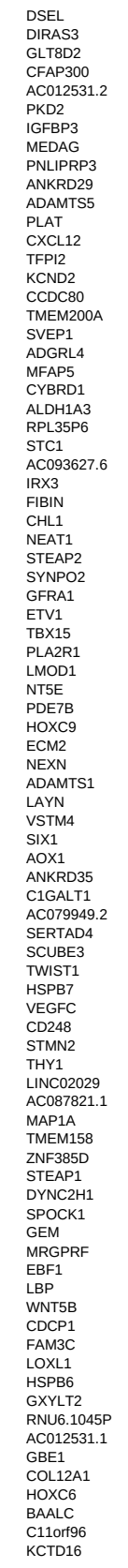

Table_S5 genes names positively enriched in MSC

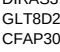

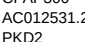

GEFPP
$M$ EDAC

PNLIPRP3
ANKRD29

ADAM

KCND2

TMEM200A

ADGRLA

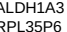

ACo93627

YYNPO2

ETX15

Hoxce
$\mathrm{ECM} 2$

NEXN

LAYN
VSTM4

AOX1

ACO79949, 2

SERTAD4
SCUBE3

HSPB7
VEGFC
VEFC

C087821.

SPOCK1

RNU6.1045

COL12A1

BAALC
C110rf96
KCTD16 


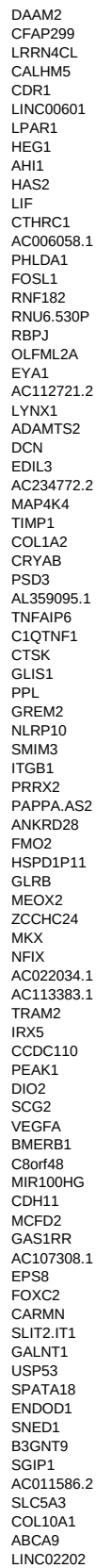

Table_S5 genes names positively enriched in MSC

LPAR1

ACO06058.

PHLDA1
FoNL1
RNF182

RNU6.530P
REPJ

OLFML2A

AC112721.2.
LCNA2
ADAMSS2
DANS

AC234772

TIMP1
COMA

CRYAB

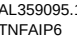

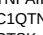

PPL
GREM

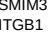

PAPPAASS
ANRR228

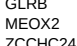

MKX
NFIX
ACO22034.I

ARAM2

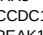

SCG2

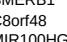

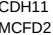

GASIRR

EPSB
OPXC2

SLIT2.IT1
GALNT1

SPATA18
ENDOD1

BBGNT
SGIP1

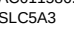

ABCA9 
COLOBA
CORB
RORB

GABRE
ABIBBP
$B$

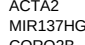

ADIRF

TLCD2

CALB2
OLFMIS
LIFME

INHBA

GDNF

FRMPDA

BDNF
NFIC

MIIFIA
PCDGAA

TAGLN

TGFB1

BDKRB1
$\mathrm{KY}$

KCNS2
ACO15922

HTRA1
CTF1
COF

2992893
$\mathrm{CHH}$

HHIPLI2
NPR3

LINCO1705
ELOVI3
OSM3

KCTD4

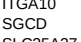

SLC25A27
ECM1

SH2DAA
EMLIIN1
LRPTM2

LRRM2
INCO119

LTRAPIL

PEXS

ANTXR1

MXRAS

SERTED4.AS
HOXC5
STTT5

SITMM10

Dok5

ARIDSB

NDP2
SNORD114.I

SNORD
FHL2

TBX2

PDZRN3
AP000525.1

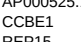

REP1
SYMC 


$$
\begin{aligned}
& \begin{array}{l}
\text { HGF } \\
\text { FRMD } \\
\text { RHOBTB3 } \\
\text { RHOB }
\end{array}
\end{aligned}
$$

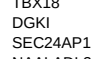

$$
\begin{aligned}
& \begin{array}{l}
\text { SEC2AAP1 } \\
\text { NAALADL2 } \\
\text { TMEM132B }
\end{array} \\
& \text { TMEM132B } \\
& \begin{array}{l}
\text { AC092807. } \\
\text { BAG2 }
\end{array} \\
& \begin{array}{l}
\text { BAG2 } \\
\text { GSTM2 } \\
\text { VASN }
\end{array} \\
& \begin{array}{l}
\text { VASN } \\
\text { POM12119P } \\
\text { ZEHXA.AS1 }
\end{array} \\
& \begin{array}{l}
\text { ZFHATA.ASI } \\
\text { CIART }
\end{array} \\
& \begin{array}{l}
\text { FAM20A } \\
\text { FGF1 } \\
\text { SLCA12 } 12 \\
\text { AC2027335 }
\end{array} \\
& \begin{array}{l}
\text { EPHA5 } \\
\text { RAB23 }
\end{array}
\end{aligned}
$$

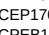

$$
\begin{aligned}
& \begin{array}{l}
\text { CPEB1 } \\
\text { EDRAA } \\
\text { DNAL11 } \\
\text { NMMT }
\end{array} \\
& \begin{array}{l}
\text { NNMT } \\
\text { ACO16397. }
\end{array} \\
& \begin{array}{l}
\text { AC016397.1 } \\
\text { ACO007673.1 } \\
\text { LUM }
\end{array}
\end{aligned}
$$

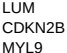

$$
\begin{aligned}
& 117 \\
& \begin{array}{l}
\text { RCAN1 } \\
\text { SCGB } \\
\text { PTCSOS2 }
\end{array} \\
& \text { KCNN21 } \\
& \begin{array}{l}
\text { EPDR1 } \\
\text { ZMAT3 }
\end{array} \\
& \begin{array}{l}
\text { BNIPBL } \\
\text { VCAN }
\end{array} \\
& \text { RNRTKP11 } \\
& \begin{array}{l}
\text { LTBP3 } \\
\text { QSOX1 } \\
\text { PPPIR3C }
\end{array} \\
& \text { SCN2A } \\
& \begin{array}{l}
\text { SCN2A } \\
\text { BMPER } \\
\text { CONA }
\end{array} \\
& \begin{array}{l}
\text { TENM4 } \\
\text { ACo5882.1. }
\end{array}
\end{aligned}
$$

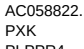

$$
\begin{aligned}
& \begin{array}{l}
\text { PLPPRA } \\
\text { POSTN } \\
\text { PoST }
\end{array} \\
& \text { Foxc } \\
& \begin{array}{l}
\text { SNX9 } \\
\text { RPSAP52 } \\
\text { SAABRAS1 }
\end{array}
\end{aligned}
$$

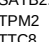

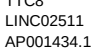

$$
\begin{aligned}
& \text { RASSF8 } \\
& \text { GRAMD2B } \\
& \begin{array}{l}
\text { SNAPA25 } \\
\text { ACo9269.1. }
\end{array} \\
& \begin{array}{l}
\text { AcCog26 } \\
\text { AsPH }
\end{array}
\end{aligned}
$$

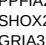

$$
\begin{aligned}
& \text { RFTN2 } \\
& \begin{array}{l}
\text { LHFPLL6 } \\
\text { RNDG.1226P } \\
\text { CASS4 }
\end{array} \\
& \begin{array}{l}
\text { CASC4 } \\
\text { SPPS }
\end{array} \\
& \begin{array}{l}
\text { SRPX } \\
\text { FAM114A1 }
\end{array}
\end{aligned}
$$


IIFBPA
ADAMTS

SERP2
ARHGAP20

SYNDIC

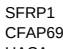

UACA

LTBP1
SETD7

HRH1
HOD3

GAS1

GGT5
PCDGB3
BHLHE41

ABCCS
SUGCT

CEMIP

TEMIP
EEF1B2P3

KCN38

EFEMP2
$\mathrm{RGS} 4$

CCDC89

SYAP1
AC112229.3
EGFR

LINCO1139

FMOD

ALL350901.2

LYPD6B

STEAP1B

AHR

THFSF4
HOXC4

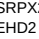

MXRA7

PNMA2

IIFFPP5
TMEM26
AP000526.1

APGF2

KGF2
KCNMA1
SMURF2

SMURF2
ACC93908.1
KTN1 


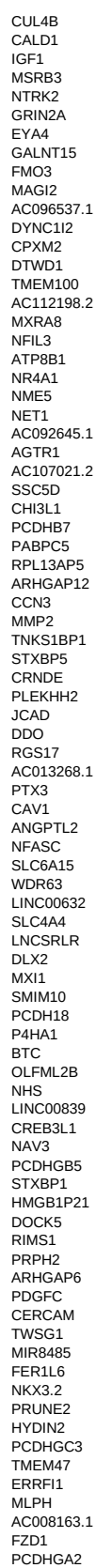




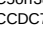

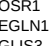

Fit44

CCPG1
PCDHGB2

ARMCA

AC073591

ACO07750

PTPRM
ACO23221.1.

REEP3

KIIA 1549

SNORD114.14 
${ }_{\text {SRGAP1 }}^{\text {IRX6 }}$

EDA2R

PCDHCCS5
FOXL11

GLS

LNCC2381
TMEM92.AS1
CAST.

CAST
LNCO1117
NPNN1

NRN1
HECW2 2
RPS12P5
FN1

$\mathrm{FN} 12.5$
ACO73115.

PDLMMS
SCARA3

TRPC4

TMEM171

NROB1
HOXC.AS2
Hess

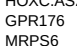

MRPS6
ANO3
FAM171B

FAM155A

SPAG9
MYO1B

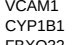

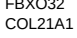

ALL35078.1
AL354811.1
KCNE4.

CHRDL1

AC0090993
SLC9A 7

SLCSAA
FAM13C
CNM221

RNF180

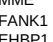

PRBP1

SLCBSAG
CACNASDI

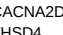

Soxg

PDE5A

GOLGA2
DYRK 3

DLC1
NDUFALLI

NDUFAAL2
C1400r132

EVC
AC138969.1
HMGA2.AS1

SLC2A10

ALL137026
CEP 290

CEP 290
SNM33
SMoc1 


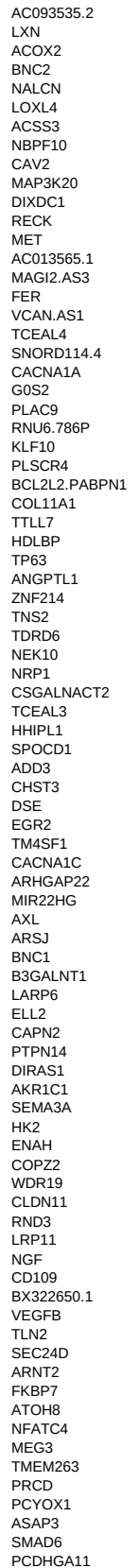




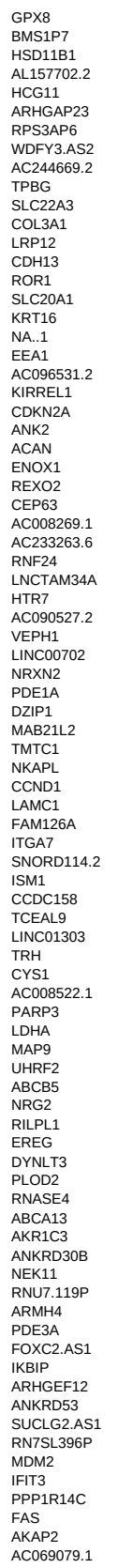

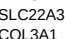

$\mathrm{COH} 13$
$\mathrm{COR1}$

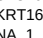

ACO96531.2

AC 0002626.1
AC233263.

RNF24
RNCTAM34A
LNCEA

ACCO90527.2
VEPH

IINCO0702

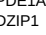

TMTC1

AAM1
FAM126A
TOM2

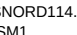

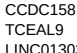

TRH
CYS1

AC008522.
PARP3

RLAS
RNA

AKR1C3

NEKR11

ARMH4

PDEBA
FOXC2.AS
IEAP

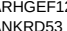

SUCLE2.AST

MDM2
IITS3

FAS 


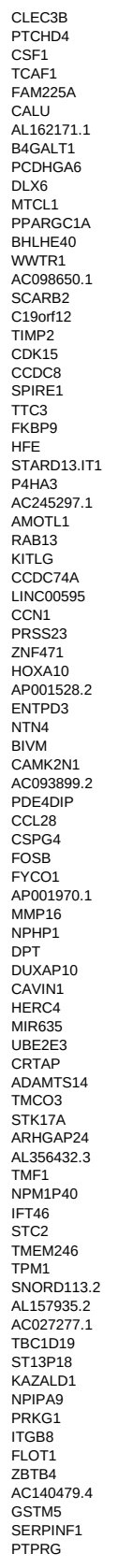

Table_S5 genes names positively enriched in MSC

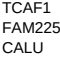

AL162171.
BAGALT1

PCDHG
DLX6

PPARGC1A
BHLHE40

ACO98650

SCARB2
C190or12

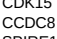

STARD13.1.

AC245297.1
AMOTL1

Incooss
CCN1

ZNF471

ENTPD3

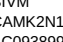

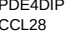

(1)

MMP16
NPHP1
DPT

DUXAP10

HERC

CRTAP

NPM1P
NTT6

STC2

TPMORD113.2

ST13P18

NPIPA

FLOT1
FLOBO

AC140479.4.
GSTM5

SERPPNF1
PTPRG 


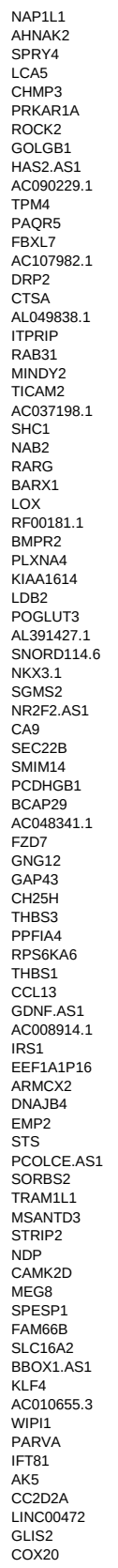

Table_S5 genes names positively enriched in MSC

SHC1

lox

LLB2

AL391427.1

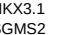

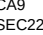

BCOP88341.

GAP43

HBS1 1

ACO08914

RS1

PCOLCE.AS

MRAM1L1

CAEGS

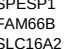

BBOX1.AS

ACO1065.3
WIP11

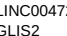




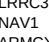
$\underset{\text { TRPA1 }}{\operatorname{ARMCX3}}$ ACA21247.1
MAPAK5

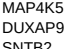

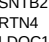

LDOC1

ALL590560.2
LINC20516
SERPINE1

PPLL6
ACO15712.2
C

TMED?.TICAM

$\mathrm{CLIP3}$
$\mathrm{D} \times 1$
$\mathrm{C}=1$

CPI
STEPG
SERINC1
SRNG

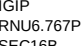

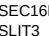

CPED1
GINM1
I

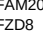

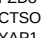

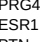

PTN
CNCN
CYP27C

RASD2
TSFE2

TGFB2
TRIO
TRIO

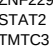

CFL2

ITCSOA

PDPBICA
PPSCA

$A R H C A P 33$
EFEMP1

Goulm4

ACC795942.2

ACD25423
CHSY3

AL021068

ALO21068
DZZP3

KIAAA217
TGEB111

MIR1245A

HMGN2P5

ACO22525.3
DEPDC7

DEPDCC
WUCATI
WCA

ERO1A

$\mathrm{EMM} \times 2$
$\mathrm{ZHX}$ 


$$
\begin{aligned}
& \begin{array}{l}
\text { SLCAA7 } \\
\text { ATP2BA } \\
\text { TLRB }
\end{array}
\end{aligned}
$$

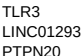

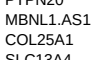

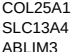

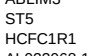

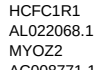

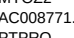

$$
\begin{aligned}
& \begin{array}{l}
\text { PTPRQ } \\
\text { MBOAT2 }
\end{array} \\
& \begin{array}{l}
\text { ACO999494.2 } \\
\text { SHISA4 } \\
\text { LSMIP1 }
\end{array} \\
& \text { AL137024.1 } \\
& \begin{array}{l}
\text { HRCT1 } \\
\text { AC10085 }
\end{array} \\
& \text { BACE1 } \\
& \begin{array}{l}
\text { EN1 } \\
\text { ACO21205.1 } \\
\text { RBMS2 }
\end{array} \\
& \begin{array}{l}
\text { RBMS2 } \\
\text { CDKL2 }
\end{array} \\
& \text { ALPI } \\
& \begin{array}{l}
\text { EVC2 } \\
\text { RCN3 } \\
\text { UNC5C }
\end{array} \\
& \text { URRELP } \\
& \begin{array}{l}
\text { CL2orf75 } \\
\text { AL117190.1 }
\end{array} \\
& \begin{array}{l}
\text { AL117190 } \\
\text { IFI6 } \\
\text { TTC3P1 }
\end{array} \\
& \begin{array}{l}
\text { CTTN } \\
\text { FAM129B } \\
\text { AC110597.3 }
\end{array} \\
& \begin{array}{l}
\text { AC110597 } \\
\text { HTR2A } \\
\text { SAMD11 }
\end{array} \\
& \begin{array}{l}
\text { SAMD1 } \\
\text { ARMCX }
\end{array} \\
& \text { ARMCX } \\
& \begin{array}{l}
\text { STAC } \\
\text { PHLDB1 } \\
\text { FGF14 }
\end{array} \\
& \begin{array}{l}
\text { FGF14 } \\
\text { SFEG }
\end{array} \\
& \begin{array}{l}
\text { ITGA1 } \\
\text { SIX4 }
\end{array}
\end{aligned}
$$

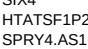

$$
\begin{aligned}
& \begin{array}{l}
\text { SPRPA.AS } \\
\text { OSPPLI } \\
\text { SECSAI }
\end{array} \\
& \begin{array}{l}
\text { SEC31A } \\
\text { PTPRK }
\end{array} \\
& \begin{array}{l}
\text { PTPRK } \\
\text { DLX3 }
\end{array} \\
& \begin{array}{l}
\text { ZNFE6 } \\
\text { NAAA2 } \\
\text { RAS }
\end{array} \\
& \begin{array}{l}
\text { ITGB5 } \\
\text { Clort198 }
\end{array} \\
& \begin{array}{l}
\text { RP1 } \\
\text { RIIIPP } \\
\text { KIFP } \\
\text { MBPSP2 } \\
\text { TPPPI } 1 \\
\text { RABGEF1 }
\end{array} \\
& \begin{array}{l}
\text { RABGEF1 } \\
\text { PRDM16 }
\end{array} \\
& \text { AL137161.1 } \\
& \begin{array}{l}
\text { PLPRN } \\
\text { DTNA } \\
\text { D }
\end{array} \\
& \begin{array}{l}
\text { DTNA } \\
\text { AFF } \\
\text { MRVII }
\end{array} \\
& \begin{array}{l}
\text { MEFFA } \\
\text { ACO27031.2 }
\end{array}
\end{aligned}
$$

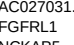

$$
\begin{aligned}
& \begin{array}{l}
\text { NCKAP5 } \\
\text { GCSPP3 } \\
\text { STX1A }
\end{array} \\
& \begin{array}{l}
\text { STIA } \\
\text { ACPP } \\
\text { LPR }
\end{array} \\
& \begin{array}{l}
\text { ACRP } \\
\text { LRRC6 } \\
\text { ZROHC7 } \\
\text { FOS } \\
\text { RBMS3 }
\end{array}
\end{aligned}
$$

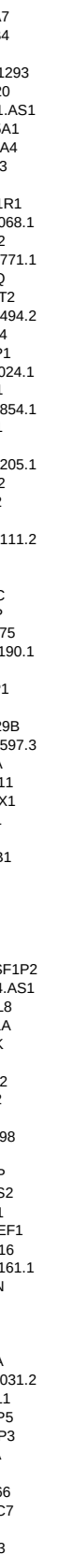




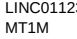

DKK1

AC105114.1

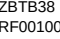

FIP1111
Al133646.1
ADDo00000.1

ADDo0090.1

TSHZ3

THSD1

SLCAAA1
PTGDS

PTESS

MRRA97HC

${ }_{\mathrm{AC} 084757}^{\mathrm{CDH} 2}$

DKK3

RMDP2

C1RL
LAMB1
MESD1

EML1

MTMRQLP
CNTLN

MMP14

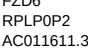

$\mathrm{LACC1} 1$
$\mathrm{UCDH}$

ZFPM2.AS1
SNTG2.AS1

SDK14

MCC
CYSTSP

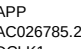

RRM28
GLPRI

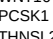

MERT

KCTD

AF127577.4

SGCE
EOGT

RASA1
SNORD13.

SNORO113
NCOA7

DCDC

POHA
CDDC149
HNRNPLI

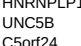

C50124
AC110048.2

DAB2

MAPPB
MAMLD
BOCD

BOC
PTPN13 


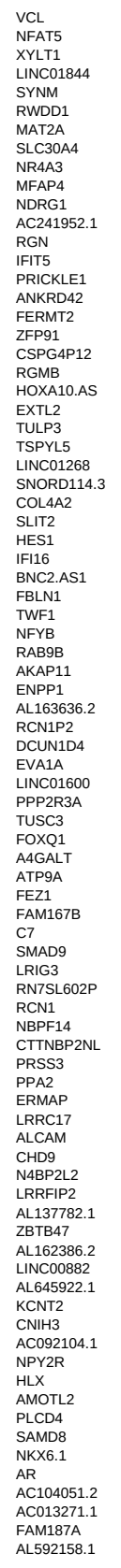

Table_S5 genes names positively enriched in MSC

NeLC

MAT2A

NR4A3
MFAP4

ACRG1

IFIT5

ANKRD42
FERMT2

2FP91

HOXA10.AS

TULP3

SNORD114.3

SLIT2
SLS1

BNC2.AS

AB99B

AL163636.2

ATP9A

SMAD9

LRIC3
RTSL602P
RNM1

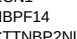

PRSS3
PPA2

RRCI7

NABPLL12

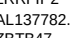

ALL162386.2

AL645922

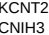

NPY2R

PLCD4

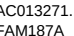




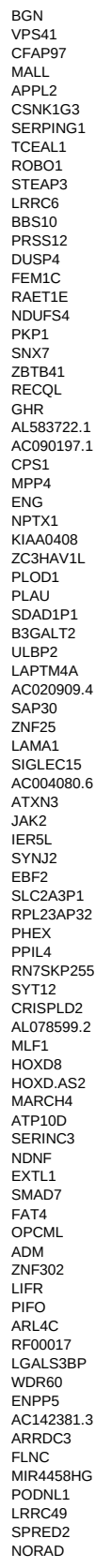

Table_S5 genes names positively enriched in MSC

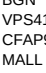

APPD2
CSNK1G3

TCEAL
RoBO

$\ln (\mathrm{RRC6})$
$\mathrm{BSS10}$

DUSP4

NDUFS4

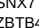

GHR

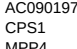

KIAAOAOB
ZCSHAV1L

PLAU

ULBP2

SAC20090.4
SNE25.
TNE25
S

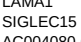

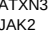

SLC CABPP
RPL23AP 32

PHEX
PPIL4

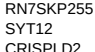

CRISPLD2
AL.078599.2

HOXXD.
HOSD

ATP10D
SERINC3

FAT4

PRLO
ARLAC

RFO0017

ENPP5

ARRDC3

LRPC4
SRRED2 


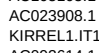

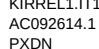

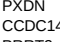

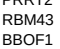

EERRO
CCNG2
TAR

CRYBG3

TAF7L
TCTN1
CAVIN3

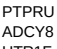

HETTL15P

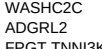

FPGT.TNNIIKK
COLSA2

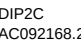

ACO92

ITGA3
RMDN2.AS
ChOS

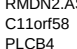

SERPNB2

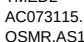

OSMR.AS
SEPTI11

AC 1037969.

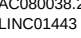

LRP1B

GIGYF2

RIIPT

AL132780.2

MPDZ
PRTECD1
ADAM9
S

sox5

SLCBA1
LNCO2595
EASTS

LNAS1955
ENORD 13.3

RARRESI
PHAX

ZNAF5
CNCA

PHYH
AlL160408.4

HOXA11

$\mathrm{LNCO1426}$
$\mathrm{PCDHA3}$

LMNA

KIAA0825
SPA617
TPD5211

HoXA7

KIAA1109 
TOPORS

FAM110B
LYPD1
MDH1B

ACOCO2053
PSG5

PSG5
ACR4
FGFP
FELN7

BDLN7

PCDHGA5
MAPPDO
ERTN2

${ }_{\mathrm{K} C \mathrm{R} K \mathrm{H}}$

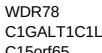

C1GALT1C
C150offr5
NCKAP1

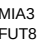

FUT8
SOBP
TTC37
TCTN2

TCTN2
CHMPBB
MARVELD

MAREED
OXLLAS
SHBPS

ATL3
ACCo8147.2
WASF

ATPRBI
COL24A1

TSPAN11
ACO79328.2

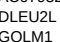

ACoog237.

ELOOVL4
LRCL2

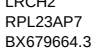

$B \times 679664.3$
ADCY6

ADCY6
CFI
STARD13

PKM

RunXI11
RKBP10
SOCS3 3

GNF22

ENPEP

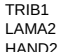

HAND2
SYNE1
ANMY3

TMAST220
Ac009237.14
SPD

SPX
ACC96636.1
RHBDL2

RTL5
RTLS

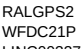

LINCO0327
SLCAAA1
SSARP

SESTD1
SNRNP27

OXR1
NR1D2
NR2

${ }_{\mathrm{TBX}}^{\mathrm{AC} 027237}$ 


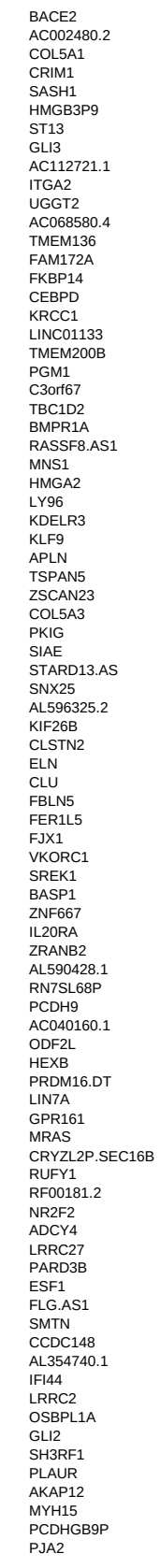

Table_S5 genes names positively enriched in MSC

$\mathrm{ST}_{\mathrm{G}} 13$
$\mathrm{GLI}$

AC112721.

UGGT2

A TMEM136
TMENG

EKEP1A

INCO1133
TMEM200B

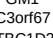

BMPP1A
RASSERAS

MNS1
HMGA2
HMA

LY96
KDERS

ZSCAN23
CO15A3

PLIG
SIAE
STAPDPBAS

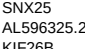

LISTI

= Jx1

BASP
BNEGG

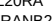

AC 040160

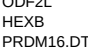

LIN7
GP161
MPAS
GAS

MRAS

RUFF1
RFFo018.2.
N20252

ADCYA

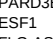

SHT.AN
SCDC148
CCDC

AL 354740.1

L 1444
LRC2

MYDH15

Page 46 


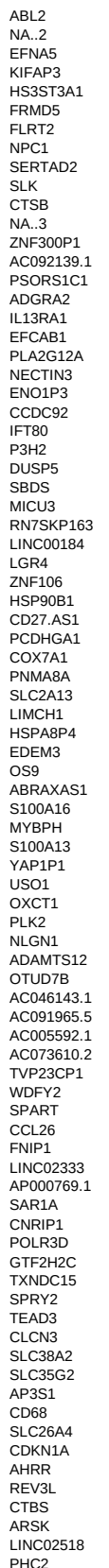

Table_S5 genes names positively enriched in MSC

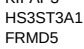

RLRT2
NPCC1

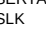

zNasoop

PSORSIC1
ADGRA2

IIIBRA

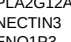

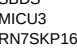

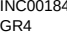

HSP90B1
CD27.AS1
COXA1

COX7A1
COMA

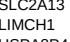

EDEM
OS9

S100A13

oxc1

NLEN1
ADAMS12
ONTST

ACCO46143
ACCO91965

ACC05592.

TVP $23 C P 1$
WDFY2
TEC

SARIA
CNRIP1

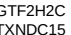

TEAD3
TCAN

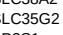

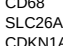

ARSK 


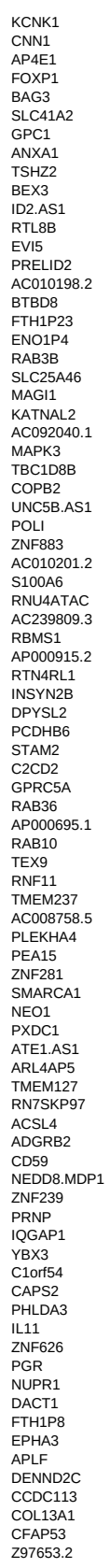

Table_S5 genes names positively enriched in MSC 


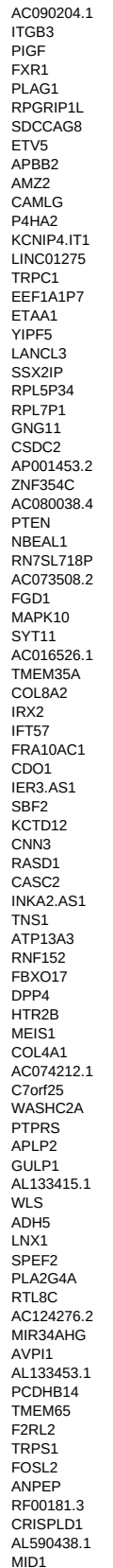

positively enriched in MSC 


$$
\begin{aligned}
& \begin{array}{l}
\text { SLITRK4 } \\
\text { CBLN3 } \\
\text { WASHC3 }
\end{array} \\
& \begin{array}{l}
\text { WASHC } \\
\text { HSPA4L }
\end{array}
\end{aligned}
$$

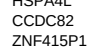

$$
\begin{aligned}
& \begin{array}{l}
\text { SEPT10 } \\
\text { RIN2 }
\end{array} \\
& \text { RIN2 } \\
& \begin{array}{l}
\text { MYO10 } \\
\text { LINC00571 }
\end{array}
\end{aligned}
$$

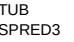

$$
\begin{aligned}
& \begin{array}{l}
\text { PLPP4 } \\
\text { GNAI1 }
\end{array} \\
& \text { TRBO } \\
& \begin{array}{l}
\text { RHOD } \\
\text { MiR604 }
\end{array} \\
& \begin{array}{l}
\text { PRAF } \\
\text { OAT }
\end{array} \\
& \begin{array}{l}
\text { ReBP } \\
\text { ACO27117 } \\
\text { P } 3 \text { H }
\end{array} \\
& \begin{array}{l}
\text { PAPSS2 } \\
\text { GPER1 }
\end{array} \\
& \begin{array}{l}
\text { METLLA } \\
\text { ACO00617.5 }
\end{array} \\
& \begin{array}{l}
\text { ALII1 } \\
\text { ANKIB1 }
\end{array} \\
& \begin{array}{l}
\text { ANKR1 } \\
\text { C220r23 } \\
\text { OPN } \\
\text { CTS } \\
\text { MSL3P1 }
\end{array}
\end{aligned}
$$

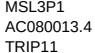

$$
\begin{aligned}
& \begin{array}{l}
\text { TRPP11 } \\
\text { ARHGE } 25
\end{array} \\
& \text { ZMYND11 } \\
& \begin{array}{l}
\text { ZSCAN30 } \\
\text { AC1094661 }
\end{array} \\
& \begin{array}{l}
\text { AL391117.1 } \\
\text { EFAB7 } \\
\text { ACO005741 }
\end{array} \\
& \begin{array}{l}
\text { ACO08574 } \\
\text { ZNF } 504
\end{array}
\end{aligned}
$$

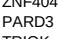

$$
\begin{aligned}
& \begin{array}{l}
\text { PAROS } \\
\text { TRPOK } \\
\text { VMMP1 }
\end{array} \\
& \begin{array}{l}
\text { VMP1 } \\
\text { MPP6 } \\
\text { NMNAT2 }
\end{array} \\
& \begin{array}{l}
\text { NPAA2 } \\
\text { SOGA3 } \\
\text { DOGG5 } \\
\text { L }
\end{array} \\
& \begin{array}{l}
\text { DLG5 } \\
\text { MAB21L1 }
\end{array} \\
& \begin{array}{l}
\text { RN7SL67P } \\
\text { POGLUT2 }
\end{array} \\
& \text { PLS3 } \\
& \text { AL } 3556968.2 \\
& \text { TMEMIAA } \\
& \begin{array}{l}
\text { SRSF11 } \\
\text { TMEMS } \\
\text { TMEMA }
\end{array} \\
& \begin{array}{l}
\text { GNDPA } \\
\text { CCDC103 } \\
\text { CCOS }
\end{array} \\
& \begin{array}{l}
\text { CCDC103 } \\
\text { ACO02094.1 } \\
\text { RALLL11A }
\end{array} \\
& { }_{S S H 1}^{\text {RALL1A }} \\
& \begin{array}{l}
\text { SPTY2D1 } \\
\text { RUBCNL }
\end{array} \\
& \begin{array}{l}
\text { HTRA3 } \\
\text { TMEM183B }
\end{array} \\
& \begin{array}{l}
\text { BCAR1 } \\
\text { ZCSHE }
\end{array} \\
& \begin{array}{l}
\text { CDKRAP1 } \\
\text { BXX70102.1 } \\
\text { B. }
\end{array}
\end{aligned}
$$

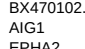

$$
\begin{aligned}
& \begin{array}{l}
\text { EPHA2 } \\
\text { SLCC55F5 } \\
\text { LNC01503 }
\end{array} \\
& \begin{array}{l}
\text { LINC01503 } \\
\text { TNN1 } \\
\text { RIPOR3 }
\end{array}
\end{aligned}
$$




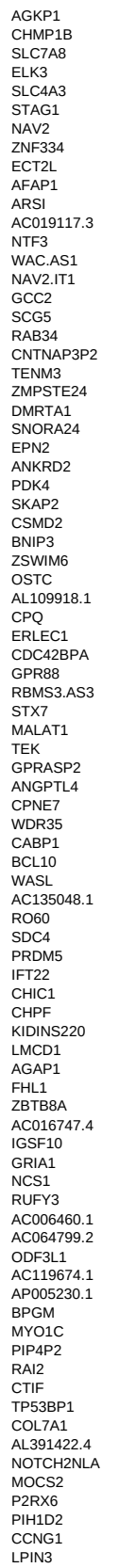

Table_S5 genes names positively enriched in MSC

SLC7AB

SLC4A3

ARSI
AC019117.

WAC.AS1

SCG5

CNTNAP3P2
TENM3

ZMPSTE24

SNORA24
EPN2

PoK4

OSTC
ALLo9918.1

ERLEC1

GPRB8
RBMS.AS

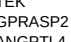

CPNE7
WOR35
WORT

Ro60

KIDINS220

ZBTBRA
AC016774

AC006460

BPGM
MYO1C

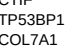

\begin{tabular}{ll} 
PHHD \\
CCNGI \\
\hline
\end{tabular} 
PCDHGAB

GATA6
GOLGA4

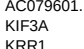

HoXA3
HRA

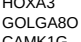

CAMK1G

SINTCAAP
PTCAR1
PEAR

PERA1
DAMM1
OAPA

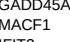

IFIT2

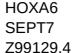

ERFE

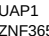

APPO242414.2
TNFAPRBL3

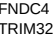

RuSC2
LAMC2

MMP3
PLXNBS3

HSDD1786
CAMSAP2

C3orfo

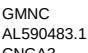

STSMS

TMESBO
ACOSA117

HSPA12A
EFR 124

NBR1

PSAP
ZEB1
CEA

IFT88
CFAP58

AC00621
HBEG
HeG

HBEGF
MAOB
MCHR1
TMED7

TMED7
ARHGAP28
RHSO

$Z \mathrm{~N} F-300$
ACO05736.1

AC ${ }^{20057}$
CMBL

GAPLINC
SLC38A5
SCHA

SLCLBAS
SPTSN1
SCNMAS

SCNAB
SLC22A23
KDELR2
KDER 
SIM2
TTNNA

NUDT6
LNCO0857

RBPMS

SAFTN4
GOLGA8Q

GOLGABO
GALNT2

AIDA
CD276
NLGNAY

NLENAY

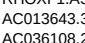

ACO36108
TAF13

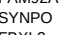

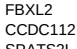

SAATS2L
ABHO2
DNAMH5

MAPG

LNCCOO842
RWDD2B

RWDP2
DSPPC
CXY

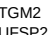

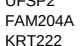

TOX2
GSC1

RAB7B

BTBDD19
RASALL

RAAAL2
STBDD1

MUC1
GOD3

CCDC47
YES1
MAN1A1

SNIBI
STSTI
PNST

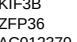

ACO12370.
OMD

OMD
TORAPIP1
TOCOPS

TMOD2
FRMDAA
FMA

FRMDAA
UBA5
MORN4

MORNA
BX842568.2
PATC

PATC
SACS

CTDSP 2
AC110792.2

RBPPAP 1

LINCO2605

NoMO1
LNCO1152

NFE2LI

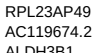

AC119674.2
ALDHB1
ADAMTS15 


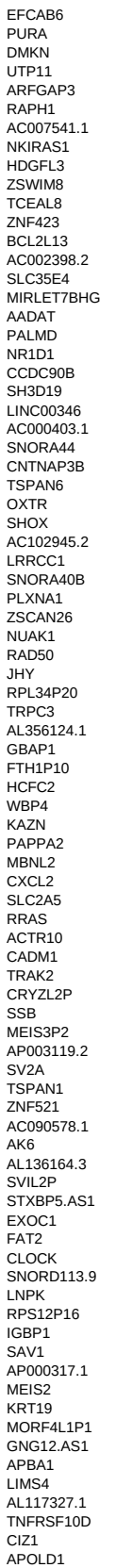


RAPS2

RABBGPP 1
CCDC188
DUYR

SH $3 B P 4$
HOXA5

FARP1

MSANTDB.TMEFF1

NuMB
NLR
SLIR

STEAP3.AS1
WEDC1

SLAIN2
STAT1
STI

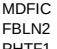

PHTF1
TANK
CSRNP3

SMPDLAA

RABGA
ATPTA

FAM16081
AL606834.1

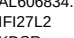

KDSR
RGSO2
LTTFI

DDIT4.AS1

$S L C 9 C 1$
$R B B P B$

TNFRSF12A

CLUAP1
ITGAB

RNU2.63P

EXOC5
NDEF2
NoP2

HBP1

WWA5A

AL136116.3
ACo68620.3

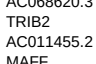

PRKAA2
DUSP6

EVIIA

HAPLN1
BDFFAS

APDoog94.1.

RNN211
ZNFFO3

TLR4
JRKL
LINC01852

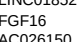

$A C 026150$
$D D X 43$

HMGBP

ACTBP7
AC CO03101.1

CCDC91
CupP3

SLHPPXD2B

GJAP1

IIC].SCHIP1
AL731568.1

SAMD9

AC008740.

PHF11

AF 165147
C20rf69

Table_S5 genes names positively enriched in MSC 
PERP
LRRC43

CCDC191
TSC2202
DIX6.AS1

CDR2L
CLX.AST

ZNAI1 2

BMP1

HOXA9
ACAD11
TGFBR2

RRAGC
AF 230666.1

AF230666
KCNQ5

KCNIK1

CNIHI

PDCFD
ACOCO0099 19

EFFGC3

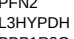

PPP1R36
SLFN12
ACO22336.

ACC22336.3
ILCST

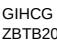

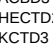

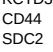

ALL550951.

AL096711.2

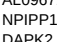

DAPK2 2
FRRT3
ERT55
ERT

NUTT12

EAHC2
SAMDOL
TTCP3

DOCK1
STAG2
CCL2

STAG2
PNMABB
CACB

AC 10065.1
AC 006249.1

AC 000249.1
RFo0019

ANKRD50

ETFDH

GRB14
PAQR7

GOLGAGLS
NEKG

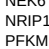

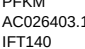

IFTIARCE1

NSA2
ALO31587.3
SYYT
STT

NOTCH2

PDLXP
STPSP
VPS $13 \mathrm{~A}$

VPSS13A
LINC00922 
MAMDC2 2

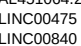

CTPP PPB

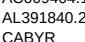

Sox6

NFE2L2

CHSTS1
KANLLIL

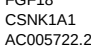

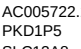

SLC12A8
TMEMB7B

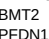

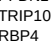

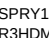

MTus2

A1 1356515

PGE
ACVR1
DDYK5

DDX5
PTPAA2

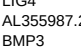

CSTA
MPV17
MPV

NOLI8
ECHDC

-15
COPDB

PHYHP
RRAGB

MEG9

MEGO
LNICOOB65
SLCOAPR1

ASAP1 1
LAMB3

ERS2

NAPB

HR
ACD12005. 2
SLCCOAA9

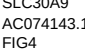


Type
Adipose MSC Number Table_S6 summary of qPCR cohort

Adipose MSC
BM-MSC

Fibroblast of
H9 ESC

HUVEC

IPS
Myoblasts

Neural Stem c

Skin fibroblast 
Table_S7 Description of Mlinc Candidates

\begin{tabular}{|c|c|c|c|c|c|c|c|c|c|c|c|c|c|c|}
\hline target_id & Sleuth qval (adj. Pval) & b (FC estimator) & length $(n t)$ & exons 0 & $\mathrm{Chr}$ Interval & Stra & $\begin{array}{l}\text { present in long } \\
\text { nd reads }\end{array}$ & Genome browser remarks & REFLNC/GTEX & $\begin{array}{l}\text { discriminative } \\
\text { kmers }\end{array}$ & $\begin{array}{l}\begin{array}{l}\text { expressed in } \\
\text { MSCs (kmers) }\end{array} \\
\end{array}$ & $\begin{array}{l}\text { strong expression } \\
\text { in SMCs (kmers) }\end{array}$ & Notable other expressions & FEELnc_coding_potential \\
\hline Mlinc.54177.1 & $5.381474218853922-10$ & 3.64181797640709 & 17139 & & \begin{tabular}{l|l}
$1867485128-67502266$ \\
\end{tabular} & & FALSE & $\begin{array}{l}\text { No annotation (close to predi } \\
\text { overexpression in fibro) }\end{array}$ & FIBRO & FALSE & Ad/Bm & TRUE & myocytes/osteoblasts & 0.336 \\
\hline Mlinc.28428.2 & $3.91322165116094 \mathrm{e}-24$ & 4.75090994700694 & 2816 & & $1288380133-88419354$ & & TRUE & TUCPITCONS_00020862 & FIBRO ONLY & TRUE & AdlBm & FALSE & $\begin{array}{l}\text { dermal papilla/muscle } \\
\text { satellites }\end{array}$ & 0.18 \\
\hline Mlinc.109628.1 & $4.18652552062097 \mathrm{e}-14$ & 3.55558466039083 & 3615 & & $6169163268-169168514$ & + & FALSE & TUCP/TCONS_00012024 (in zone) & $\begin{array}{l}\text { FIBRO/ARTERYI } \\
\text { BioMarker sunvie }\end{array}$ & FALSE & Ad/Bm/uc & TRUE & $\begin{array}{l}\text { myocyles/chondrocytes/ } \\
\text { astrocytes/preadipocytes }\end{array}$ & 0.446 \\
\hline \begin{tabular}{|l} 
Mlinc.128022.2 \\
Mlinc.32708.1
\end{tabular} & \begin{tabular}{|l|}
$\frac{2.244400524338899-15}{0.00166963056755997}$ \\
\end{tabular} & $\begin{array}{l}\frac{3.19030456476542}{2.11405992530657} \\
\end{array}$ & $\begin{array}{r}992 \\
4346\end{array}$ & & $\begin{array}{c}9115596550-115692288 \\
1375559960-75643205\end{array}$ & & $\begin{array}{l}\text { TRUE } \\
\text { FALSE }\end{array}$ & $\begin{array}{l}\text { TuCP/TCONS 00016469 (lot of variants in } \\
\text { zone) + +umerous chromatin marks } \\
\text { In close } 3 \text { ' region of LoM7 }\end{array}$ & $\begin{array}{l}\text { FIBRO ONLY } \\
\text { BRAIN (ISoform ?) }\end{array}$ & \begin{tabular}{|l} 
TRUE \\
FALSE
\end{tabular} & \begin{tabular}{|l|l}
$\mid A d / B m$ \\
$A d / B m / U C$
\end{tabular} & \begin{tabular}{|l} 
TRUE \\
TRUE
\end{tabular} & \begin{tabular}{|l} 
Myocytes/myoblastes \\
Chondro (very strong) \\
\end{tabular} & \begin{tabular}{|l|l}
0.268 \\
0.118
\end{tabular} \\
\hline Minc.10317.1 & $8.78516928066226 \mathrm{e}-11$ & 3.69104237801407 & 8760 & & $1215239087-215247846$ & + & FALSE & $\begin{array}{l}\text { In close } 3^{\prime} \text { 'region of KCNK2, very high } \\
\text { expression in fibro }\end{array}$ & & TRUE & Ad/Bm & EALSE & $\begin{array}{l}\text { Cnondio (very strong) } \\
\text { osteo/chondro }\end{array}$ & 0.118 \\
\hline Mlinc.45246.1 & $5.52855779835755 \mathrm{e}-11$ & 3.12467428011448 & 3825 & & 16 65129303-65133127 & + & FALSE & TUCP/TCONS_00024436 (partial overlappig) & & FALSE & Ad/Bm/UC & TRUE & $\begin{array}{l}\text { SMc (very } \\
\text { stronglosteoblast (very } \\
\text { strong) }\end{array}$ & 0.156 \\
\hline \begin{tabular}{|l|} 
Mlinc.61271.1 \\
Mlinc.6947.1 \\
\end{tabular} & $\begin{array}{l}0.000934727743803686 \\
0.0887756655506394\end{array}$ & $\begin{array}{l}2.11029387027848 \\
1.7936396004482\end{array}$ & 4902 & & $246810683-46815584$ & + & $\begin{array}{l}\text { FALSE } \\
\text { FALSE }\end{array}$ & \begin{tabular}{|l} 
TUCP/TCONS_ 00003666, partial overlapping \\
On repeated region
\end{tabular} & $\begin{array}{l}\text { FFBRR/MUSCLE/ } \\
\text { ADIPO/Blood }\end{array}$ & $\begin{array}{l}\text { FALSE } \\
\text { FALSE }\end{array}$ & \begin{tabular}{|l|} 
Ad/Bm/UC \\
no expression
\end{tabular} & TRUE & $\begin{array}{l}\begin{array}{l}\text { Osteoblast (very strong), } \\
\text { chondrocytes (very strong) }\end{array} \\
\end{array}$ & $\frac{0.126}{0.0348}$ \\
\hline \begin{tabular}{|l|} 
Mlinc.89912.1 \\
Mlinc.98481.1
\end{tabular} & \begin{tabular}{|l|}
$2.19410912061805 \mathrm{e}-13$ \\
$4.045883114693 \mathrm{e}-14$ \\
\end{tabular} & $\begin{array}{l}3.1608578751305 \\
3.434452529039\end{array}$ & $\begin{array}{l}3155 \\
1532\end{array}$ & & $\begin{array}{l}480263395-80266553 \\
51120687803-120689334\end{array}$ & & TRUE & $\begin{array}{l}\text { In close } 5^{\prime} \text { region of FGF5 (very high in fibro)! } \\
\text { Zone of regulation }++\end{array}$ & & TRUE & Ad/Bm/uc & TRUE & $\begin{array}{l}\begin{array}{l}\text { muscle } \\
\text { satellit/papilla/myocytes } \\
\text { myoblastlchondro }\end{array} \\
\end{array}$ & 0.198 \\
\hline \begin{tabular}{|l|} 
Mlinc.123973.1 \\
Mlinc.63232.1 \\
\end{tabular} & \begin{tabular}{|l|}
$1.61744832508345 \mathrm{e}-09$ \\
$1.85061125320116 \mathrm{e}-11$ \\
\end{tabular} & $\begin{array}{l}2.80103674598808 \\
3.24619016103911\end{array}$ & $\begin{array}{l}494 \\
232\end{array}$ & & $\begin{array}{l}912823444-128239007 \\
292068044-202038275 \\
\end{array}$ & + & $\begin{array}{l}\text { FALSE } \\
\text { FALSE }\end{array}$ & $\begin{array}{l}\text { In close } 3^{\prime} \text { 'region of PRR16 (very high in fibro) } \\
\text { In close } 3^{\prime} \text { region of LURAP1L (high in fibro) }\end{array}$ & & FALSE & Ad/Bm & $\begin{array}{l}\text { TRUE } \\
\text { TRUE }\end{array}$ & $\begin{array}{l}\text { myobalasichonoro } \\
\text { hepatocytes/osteo (very } \\
\text { strong) }\end{array}$ & 0.244 \\
\hline Mlinc & $1.43285225448328 \mathrm{e}-06$ & 2.9079538 & 8953 & & $\begin{array}{l}292068044-92068275 \\
6130445023-130453975\end{array}$ & + & $\begin{array}{l}\text { FALSE } \\
\text { FALSE }\end{array}$ & $\begin{array}{l}\text { repetition } \\
\text { In close } 3 \\
\end{array}$ & $\begin{array}{l}\text { OVVELLAPII } \\
\text { PARTALL }\end{array}$ & $\begin{array}{l}\text { FALSE } \\
\text { FALSE }\end{array}$ & Ad/Bm & TRUE & \begin{tabular}{|l} 
Myocytes/myoblast//SMC \\
hepatocytes
\end{tabular} & 0.328 \\
\hline \begin{tabular}{|l|} 
Mlinc.117098.1 \\
\end{tabular} & 9.0141591 & 2.68354574301863 & 1359 & & $8299352-300710$ & & FALSE & lincRNA AC136777.1 i i zone (10Kb) & & FALSE & Ad/Bm & FALSE & 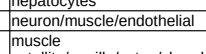 & 0.106 \\
\hline Minc.70094.1 & $5.13172839816861 \mathrm{e}-10$ & 2.8220665603735 & 946 & te & $2237319841-237320786$ & & FALSE & f COL613 (very high in fibro) & & TRUE & Ad/Bm & FALSE & & ro.12 \\
\hline Mlinc.64225.1 & 0.002065 & 2.01363639988553 & 302 & & $2113609969-113610270$ & & TRUE & $\begin{array}{l}\text { LRT close to RPL2APP7 (pssudogene + } \\
\text { strong repetition (high in fibro) }\end{array}$ & & FALSE & $\mathrm{Bm}$ & TRUE & & 0.368 \\
\hline nc.54155.1 & 1.003412 & 2.896783 & 5908 & 1 & $1867389037-67394944$ & & FALSE & In intron of & & FALSE & Ad/Bm & TRUE & osteo/hESC/chondro & 0.0402 \\
\hline \begin{tabular}{|l} 
Milinc. 78582.1 \\
Minc.109600.1
\end{tabular} & \begin{tabular}{|l|l|}
$1.6065155850913348 \mathrm{e}-09$ \\
$1.23389178444261 \mathrm{e}-08$
\end{tabular} & $\begin{array}{l}\frac{3.3262627797663159}{2.57894271954655} \\
\end{array}$ & 3402 & & 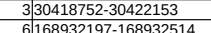 & & $\begin{array}{l}\text { FALSE } \\
\text { FALSE }\end{array}$ & 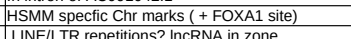 & & $\frac{N A}{\text { FALSE }}$ & NA & NA & NA & 0.178 \\
\hline Mlinc. 9300.1 & $163418031048994 \mathrm{e}-08$ & 266341118521261 & (3882 & & 1 1924886616-192489997 & & FALSE & 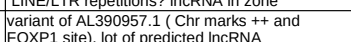 & & FALSE & Ad/Bm/UC & TRUE & SMC (very strong) & 0.144 \\
\hline Mlinc.54554.1 & $2.57868369698677 \mathrm{e}-07$ & 2.45953847799982 & 2156 & & $1875463916-75466071$ & + & FALSE & HSMM specfic Chr marks (+FOXA1 Site) & & FALSE & Bm/uc & TRUE & $\begin{array}{l}\text { hepatocyes/smc (very } \\
\text { strong/muscle cell }\end{array}$ & 0.144 \\
\hline Mlinc.83437.1 & $3.75186606665131 \mathrm{e}-11$ & 2.92026510167055 & 2904 & 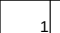 & $3126213050-126215953$ & + & FALSE & ir Marks & & FALSE & Ad/Bm/UC & FALSE & $\begin{array}{l}\text { mesenchliali/neural } \\
\text { prog.myocytes/epithelial }\end{array}$ & 0.292 \\
\hline Mlinc.127918.1 & $859549299747758 \mathrm{e}-05$ & 22148444399395 & 2870 & & $113624677-113628441$ & + & FALSE & ark, TF binding & & TRUE & $\mathrm{Ad} / \mathrm{Bm}$ & FALSE & Chondro/panilla & 0.302 \\
\hline Mlinc.9231.1 & $2.09587353035184 \mathrm{e}-06$ & 2.77869731979218 & 2415 & & 1186681846-186684260 & & FALSE & $\begin{array}{l}\text { close to an AS of PTGS2 antisense NFKB1 } \\
\text { complex +t+: Numerous Chr mark + TF } \\
\text { binding sites }\end{array}$ & & FALSE & Ad/Bm/UC & TRUE & $\begin{array}{l}\text { Mononuclear cells (very } \\
\text { strong) }\end{array}$ & 0.152 \\
\hline Mlinc.78418.1 & $1.82921400272116 \mathrm{e}-05$ & 2.52537568052055 & 2945 & 1 & 3 27646119-27649063 & + & FALSE & 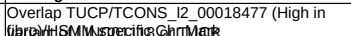 & & FALSE & $\mathrm{Ad} / \mathrm{Bm}$ & FALSE & epithelia/myocytes/chondro & 0.232 \\
\hline Mlin & 0.00 & 1.85 & 8834 & & 246 & $I^{+}$ & FAL & $\begin{array}{l}\text { TCONS =0003666, rich zone in lincRNA, Chr } \\
\text { mark t TF binding site, close to SOCS5 (high in } \\
\text { fibro ++) }\end{array}$ & & TRUE & Ad/Bm & & oster & 0.0162 \\
\hline \begin{tabular}{|l|l} 
Mlinc.111761.1 \\
Milc 51561
\end{tabular} & $\begin{array}{ll}0.0004296 \\
276418\end{array}$ & \begin{tabular}{|l}
2.16878061892736 \\
3550255005675
\end{tabular} & 1232 & 1 & $\begin{array}{r}738054085-38055316 \\
86735276701566\end{array}$ & & $\begin{array}{l}\text { FALSE } \\
\text { FAlSE }\end{array}$ & 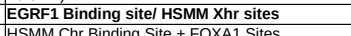 & & $\begin{array}{l}\text { FALSE } \\
\text { TRUE }\end{array}$ & BM & $\frac{T}{T \text { TUE }}$ & 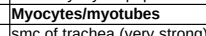 & 0.084 \\
\hline Mlinc.4751523.1 & 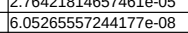 & 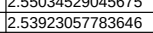 & $\frac{6240}{1558}$ & & $\begin{array}{l}186139532-1-64401566 \\
1713490975-13492532\end{array}$ & & $\begin{array}{l}\text { FALLE } \\
\text { FALSE }\end{array}$ & $\begin{array}{l}\text { HSMM Chr Bininging Site + FOXA1 S Sites } \\
\text { In 3' of gene HS3STSA1 (High in fibro) }\end{array}$ & FIBRO/ DIFFUSE & $\frac{\mid \text { RAUE }}{\text { FALSE }}$ & $\begin{array}{l}\text { Adale } \\
\text { Ad }\end{array}$ & $\begin{array}{l}\text { TRUE } \\
\text { TRUE }\end{array}$ & $\begin{array}{l}\text { smct of tracheaea (verys } \\
\text { epithelial/mesanglial }\end{array}$ & 0.3342 \\
\hline Minc.18093.1 & $3.3005434599755 \mathrm{e}-09$ & 2.70413881781352 & 977 & & \begin{tabular}{l|l|}
11 & $12085459-12086435$ \\
\end{tabular} & & FALSE & $\begin{array}{l}\text { Close to AC124276.2 var } \\
\text { TT binding site }++++\end{array}$ & & FALSE & Ad/uC & TRUE & epithalial/keratinocytes & 0.236 \\
\hline Minc.57835.1 & 1.15589458808076e-08 & 2.42525523068851 & 927 & & \begin{tabular}{l|l|}
$194737723-44738649$ \\
\end{tabular} & & FALSE & TUCP/TC & & FALSE & FALSE & FALSE & $\mid \begin{array}{l}\text { Mononucuc } \\
\text { strong) }\end{array}$ & 0.436 \\
\hline Mlinc.104634.1 & 7.05793182343196e-12 & 3.12285461836103 & 2731 & & $657260477-57263207$ & & FALSE & $\begin{array}{l}\text { TUCPTCONS_00011222 +C } \\
\text { and TF binding site }\end{array}$ & & FALSE & Ad/Bm/UC & TRUE & SMC (strong) & 0.314 \\
\hline Mlinc.80687.1 & 3.475299 & 2.1578 & 537 & 1 & 36501 & + & FAL & In Intron of gene ADAM-AS & & & BM & & ndro/neural & 0.464 \\
\hline Mlinc.966 & 2.09413 & 2.08136 & 637 & & $5[844919$ & & FALSE & & & FALSE & BMIUS & FALSE & myocytes/osteo/chondro & \\
\hline
\end{tabular}




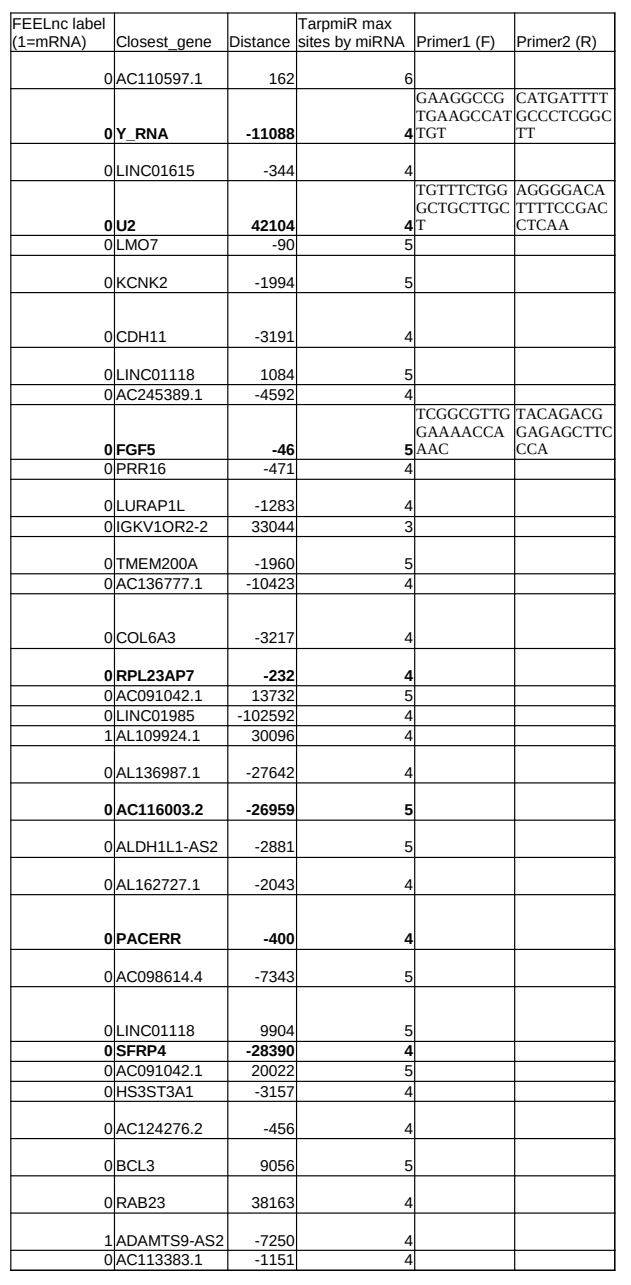




\begin{tabular}{|c|c|c|c|c|c|}
\hline $\begin{array}{l}\text { Minc.2842 } \\
\text { Uniprot ID }\end{array}$ & & $\begin{array}{l}\text { Minc. } 12802 \\
\text { Uniprot ID }\end{array}$ & & $\begin{array}{l}\text { Mlinc.8991 } \\
\text { Uniprot ID }\end{array}$ & \\
\hline $\begin{array}{l}\text { Q496A3 } \\
\text { O9UBBM1 }\end{array}$ & $\begin{array}{l}\text { SPATS1 } \\
\text { PEMT }\end{array}$ & $\begin{array}{l}\text { P31994 } \\
\text { O992T5 }\end{array}$ & $\begin{array}{l}\text { FCGR2B } \\
\text { GRP52 }\end{array}$ & $\begin{array}{l}\text { P02760 } \\
\text { O992830 }\end{array}$ & AMBP \\
\hline 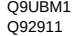 & $\begin{array}{l}\text { PETT } \\
\text { SLC5A5 }\end{array}$ & 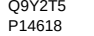 & $\begin{array}{l}\text { GRR52 } \\
\text { PKM }\end{array}$ & $\begin{array}{l}\text { Q928280 } \\
\text { Q6NU16 }\end{array}$ & $\begin{array}{l}\text { KATAA } \\
\text { CHADL }\end{array}$ \\
\hline P09001 & MRPL3 & Q9P1W8 & SIRPG & Q9Y2K9 & STXBP5L \\
\hline $\begin{array}{l}\text { Q996625 } \\
\text { P20700 }\end{array}$ & $\begin{array}{l}\text { MED8 } \\
\text { LMNB1 }\end{array}$ & $\begin{array}{l}\text { Q9P0011 } \\
\text { Q8N910 }\end{array}$ & $\begin{array}{l}\text { PDP1 } \\
\text { SYT2 }\end{array}$ & $\begin{array}{l}\text { Q9N2929 } \\
\text { Q8N6M3 }\end{array}$ & $\begin{array}{l}\text { TMEM100 } \\
\text { FITM2 }\end{array}$ \\
\hline Q5FYBO & ARSJ & Q86×59 & C17orf82 & 043915 & VEGFD \\
\hline 932 & $\begin{array}{l}\text { KIF3B } \\
\text { DXO }\end{array}$ & $\begin{array}{l}\text { Q8NCL8 } \\
\text { O9P2T1 }\end{array}$ & $\begin{array}{l}\text { TMEM116 } \\
\text { GMPR2 }\end{array}$ & $\begin{array}{l}\text { Q9NRDD1 } \\
\text { O9USH8 }\end{array}$ & $\begin{array}{l}\text { FBBO6 } \\
\text { METRN }\end{array}$ \\
\hline Q9P232 & CNTN3 & 075581 & & Q9BWW8 & APOL6 \\
\hline & $\begin{array}{l}\text { INIP } \\
\text { TRAP1 }\end{array}$ & $\begin{array}{l}\text { Q7L591 } \\
\text { O616999 }\end{array}$ & $\begin{array}{l}\text { DOK3 } \\
\text { OR10K2 }\end{array}$ & $\begin{array}{l}\text { Q15746 } \\
\text { O9BOEF }\end{array}$ & $\begin{array}{l}\text { MYLK } \\
\text { TUBA1C }\end{array}$ \\
\hline $\begin{array}{l}\text { QOD2K2 } \\
\text { QOD2 }\end{array}$ & KLHL30 & $\begin{array}{l}\text { Q9uM11 } \\
\text { Qum }\end{array}$ & FZR1 & $\begin{array}{l}\text { Q96HE7 } \\
\text { Q }\end{array}$ & ERO1A \\
\hline Q16891 & IMMT & Q8WUH6 & TMEM263 & Q8TCG2 & PIAK $2 B$ \\
\hline & $\begin{array}{l}\text { CSNK163 } \\
\text { MAN2A1 }\end{array}$ & $\begin{array}{l}\text { Q9UKNN8 } \\
\text { Q966A77 }\end{array}$ & $\begin{array}{l}\text { GTF3C4 } \\
\text { ISL2 }\end{array}$ & $\begin{array}{l}\text { Q999969 } \\
\text { O433148 }\end{array}$ & $\begin{array}{l}\text { RARRES2 } \\
\text { RNMT }\end{array}$ \\
\hline Q99946 & $\begin{array}{l}\text { PRTI } \\
\text { PRT }\end{array}$ & $\begin{array}{l}\text { Q5ULJ6 } \\
\text { Q }\end{array}$ & ZMIZ1 & $\begin{array}{l}\text { Q7726V5 } \\
\end{array}$ & ADAT2 \\
\hline $\begin{array}{l}\text { Q996JJ6 } \\
\text { O88zH2 }\end{array}$ & & $\begin{array}{l}\text { Q99829 } \\
\text { O29983 }\end{array}$ & $\begin{array}{l}{ }_{\text {MINEA }} \\
\text { MICA }\end{array}$ & $\begin{array}{l}\text { Q9U1A99 } \\
\text { PPBS68 }\end{array}$ & $\begin{array}{l}\text { XPO7 } \\
\text { CYP3A4 }\end{array}$ \\
\hline Q8NBF1 & GLIS1 & $\begin{array}{l}\text { Q92783 } \\
\text { Q9283 }\end{array}$ & $\begin{array}{l}\text { STAM } \\
\text { STA }\end{array}$ & Q8N135 & LGI4 \\
\hline $\begin{array}{l}\text { Q99BXX6 } \\
\text { O8TTBA4 }\end{array}$ & $\begin{array}{l}\text { RAB11FIP5 } \\
\text { MGARP }\end{array}$ & $\begin{array}{l}\text { Q81YX7 } \\
\text { PODN77 }\end{array}$ & $\begin{array}{l}\text { SAXO1 } \\
\text { OPN1MWE }\end{array}$ & $\begin{array}{l}\text { Q96A04 } \\
\text { OO99271 }\end{array}$ & \\
\hline $\begin{array}{l}\text { Q89HB4 } \\
\text { Q9H } 251\end{array}$ & $\mathrm{CDH} 23$ & PODN78 & OPN1MW3 & Q8NAE3 & $\begin{array}{l}\text { AMELX } \\
\text { LINC0155 }\end{array}$ \\
\hline $\begin{array}{l}\text { Q966RB3 } \\
\text { Q9HBRO }\end{array}$ & $\begin{array}{l}\text { MCCC1 } 1 \\
\text { SLC38A10 }\end{array}$ & $\begin{array}{l}\text { Q6ZMMH5 } \\
05 T \text { STC9 }\end{array}$ & $\begin{array}{l}\text { SLCCB9A } \\
\text { ARFGEF }\end{array}$ & $\begin{array}{l}\text { O604003 } \\
\text { P49908 }\end{array}$ & $\begin{array}{l}\text { OR1OH2 } \\
\text { SELENOP }\end{array}$ \\
\hline $\begin{array}{l}\text { Q9BTU6 } \\
\text { Q9BTE }\end{array}$ & P14K2A & $\begin{array}{l}\text { P04062 } \\
\text { Pothe }\end{array}$ & GBA & Q9Y4C5 & CHST2 \\
\hline $\begin{array}{l}\text { Q99Y2P8 } \\
\text { O15056 }\end{array}$ & $\begin{array}{l}\text { RCL1 } \\
\text { EIF4H }\end{array}$ & $\begin{array}{l}\text { Q8IVM8 } \\
\text { O96788 }\end{array}$ & $\begin{array}{l}\text { LLC2LA9 } \\
\text { UHRF1 }\end{array}$ & $\begin{array}{l}\text { Q1414242 } \\
\text { P30085 }\end{array}$ & $\begin{array}{l}\text { TRIMP14 } \\
\text { CMPR1 }\end{array}$ \\
\hline $\begin{array}{l}\text { Q99UMB } 22 \\
\text { O9GZP1 }\end{array}$ & $\begin{array}{l}\text { SYIRG } \\
\text { NRSN2 }\end{array}$ & $\begin{array}{l}\text { Q5SWL18 } \\
\text { Q8NA27 }\end{array}$ & $\begin{array}{l}\text { PRAMEF } 19 \\
\text { NME8 }\end{array}$ & $\begin{array}{l}\text { G3VOH } \\
\text { P60880 }\end{array}$ & $\begin{array}{l}\text { SLCO1B } \\
\text { SNAP25 }\end{array}$ \\
\hline $\begin{array}{l}\text { POC645 } \\
\text { Poc1 }\end{array}$ & ORAE1 & Q8NBP7 & PCSK9 & P01767 & IGHV3-53 \\
\hline $\begin{array}{l}\text { Q6ZZVL6 } \\
\text { O775009 }\end{array}$ & $\begin{array}{l}\text { KIAA15 } \\
\text { CNNK }\end{array}$ & $\begin{array}{l}\text { Q87CXX1 } \\
\text { O137072 }\end{array}$ & $\begin{array}{l}\text { DYNC2LI } \\
\text { BAGE }\end{array}$ & $\begin{array}{l}\text { Q8WUJ1 } \\
\text { O6ZONON7 }\end{array}$ & $\begin{array}{l}\text { CYB5D2 } \\
\text { SLCOAC1 }\end{array}$ \\
\hline $\begin{array}{l}\text { Q99574 } \\
\text { Q }\end{array}$ & SERPINI1 & 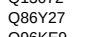 & $\begin{array}{l}\text { BAAE5 } \\
\text { PTSP }\end{array}$ & P83731 & $\begin{array}{l}\text { RPLL24 } \\
\text { FPL24 }\end{array}$ \\
\hline $\begin{array}{l}\text { Q9955G7 } \\
\text { Q1A5X6 }\end{array}$ & $\begin{array}{l}\text { PCDHGA6 } \\
\text { IQCJ }\end{array}$ & $\begin{array}{l}\text { 9966K9 } \\
\text { Q6UXस8 }\end{array}$ & $\begin{array}{l}\text { BRBD6 } \\
\text { CCBE1 }\end{array}$ & $\begin{array}{l}\text { Q5T11111 } \\
\text { P05496 }\end{array}$ & $\begin{array}{l}\text { EYS } \\
\text { ATP5MC1 }\end{array}$ \\
\hline , & MFSD6L & $\begin{array}{l}\text { Q14409 } \\
\text { p56177 }\end{array}$ & GK3P & $\begin{array}{l}\text { P78312 } \\
P\end{array}$ & FAM193A \\
\hline & & & & & \\
\hline $\begin{array}{l}\text { Q8NNG63 } \\
\text { O14950 }\end{array}$ & $\begin{array}{l}\text { ARFGAP1 } \\
\text { MYL12B }\end{array}$ & 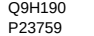 & $\begin{array}{l}\text { SDCBPP2 } \\
\text { PAX7 }\end{array}$ & $\begin{array}{l}\text { Q15834 } \\
\text { Q111201 }\end{array}$ & $\begin{array}{l}\text { CCDCDSBB } \\
\text { STBGAL1 }\end{array}$ \\
\hline 2028 & MACC1 & 015409 & FOXP2 & Q9Y6H1 & CHCHD2 \\
\hline & ED2 & 60520 & GABARA & & TUBA1B \\
\hline 釸6 & $\begin{array}{l}\text { RRRQ4 } \\
\text { GALS1 }\end{array}$ & 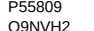 & $\begin{array}{l}\text { XXCT1 } \\
\text { NTS7 }\end{array}$ & $\begin{array}{l}\text { Q4944X3 } \\
0966.177\end{array}$ & ZNF404 \\
\hline $\begin{array}{l}\text { P59536 } \\
\text { Pons }\end{array}$ & TAS2R41 & $\begin{array}{l}\text { Q96NV12 } \\
\text { Q8NOZZ }\end{array}$ & PUSL1 & $\begin{array}{l}\text { 9966J7 } \\
\text { Q86xS8 }\end{array}$ & $\begin{array}{l}\text { DHRS1 } \\
\text { RNF130 }\end{array}$ \\
\hline O959c & & $\begin{array}{l}\text { Q7L4S7 } \\
\text { OaY556 }\end{array}$ & $\begin{array}{l}\text { ARMCX6 } \\
\end{array}$ & P48742 & 0 \\
\hline $\begin{array}{l}\text { Q959593 } \\
\text { P21439 }\end{array}$ & $\begin{array}{l}\text { TBB55 } \\
\text { ABCB44 }\end{array}$ & $\begin{array}{l}\text { Q9YY556 } \\
\text { Q8WUI4 }\end{array}$ & $\begin{array}{l}\text { TIMM10B } \\
\text { HDAC7 }\end{array}$ & PoDMvo & CT45A7 \\
\hline $\begin{array}{l}P 2007 \\
\text { P20TC }\end{array}$ & $\begin{array}{l}\text { ANXA7 } \\
\text { a }\end{array}$ & $\mathrm{PO}$ & KRT6B & & ZNF709 \\
\hline $\begin{array}{l}\text { Q8TTCC3 } \\
\text { Q60788 }\end{array}$ & $\begin{array}{l}\text { MRPP30 } \\
\text { APOA5 }\end{array}$ & $\begin{array}{l}\text { Q8IISS8 } \\
\text { O95455 }\end{array}$ & $\begin{array}{l}\text { BDD1L2 } \\
\text { TGDS }\end{array}$ & 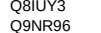 & $\begin{array}{l}\text { GLAMD } \\
\text { TLR9 }\end{array}$ \\
\hline Q13443 & ADAM9 & Q969P6 & TOP1MT & Q8NBTO & POC1A \\
\hline $\begin{array}{l}\text { Q7LL111 } \\
\text { O996906 }\end{array}$ & $\begin{array}{l}\text { ARMCX2 } \\
\text { PPP2R3C }\end{array}$ & $\begin{array}{l}\text { Q3MPIP1 } \\
\text { Q86PV6 }\end{array}$ & $\begin{array}{l}\text { ITPRIIILL2 } \\
\text { MYL4 }\end{array}$ & $\begin{array}{l}014827 \\
017855\end{array}$ & $\begin{array}{l}\text { RASGRF } 2 \\
\text { FAMR878 }\end{array}$ \\
\hline Q8N1B4 & VPS52 & P04001 & OPN1MW & $\begin{array}{l}\text { Q9Hом5 } \\
\text { Q }\end{array}$ & ZNF700 \\
\hline & ASN & & & & \\
\hline & $\begin{array}{l}\text { DNN } \\
\text { SRS }\end{array}$ & & & $\begin{array}{l}\text { Q990V3 } \\
\text { O04771 }\end{array}$ & SH3BP4 \\
\hline $\begin{array}{l}2{ }^{2} 34 \\
\text { DV7 }\end{array}$ & $\begin{array}{l}\text { SRRF5 } \\
\text { TNRC6A }\end{array}$ & $\begin{array}{l}\text { Q16566 } \\
\text { Q5JTZ5 }\end{array}$ & $\begin{array}{l}\text { NRF1 } \\
\text { C9ort152 }\end{array}$ & $\begin{array}{l}\text { O04771 } \\
\text { Q6ZRS4 }\end{array}$ & $\begin{array}{l}\text { ACVR1 } \\
\text { ITPRID1 }\end{array}$ \\
\hline & MUC1 & 095382 & $P P F$ & & MARCH6 \\
\hline $\begin{array}{l}\text { Q966AN5 } \\
\text { O9gBXT2 }\end{array}$ & $\begin{array}{l}\text { TMEM1433 } \\
\text { CACNG6 }\end{array}$ & $\begin{array}{l}\text { Q2QDD12 } \\
\text { O9279796 }\end{array}$ & $\begin{array}{l}\text { RELL1 } \\
\text { DLG3 }\end{array}$ & $\begin{array}{l}\text { Q131103 } \\
\text { O75663 }\end{array}$ & $\begin{array}{l}\text { SPP2 } \\
\text { TIPRL }\end{array}$ \\
\hline & & & ULG & Q8NBM4 & UBAC2 \\
\hline & & & AMM50 & Q8NEGO & \\
\hline & & & J3F2 & & \\
\hline & & & & Q7 & GDP \\
\hline & $\begin{array}{l}\text { CSP } \\
\text { HMB }\end{array}$ & & & j36 & Th \\
\hline P54922 & ADPRH & P21397 & MAOA & 19 & ZFP69B \\
\hline Q965A4 & SERINC2 & & & & \\
\hline $\begin{array}{l}\text { Q7754945 } \\
\text { Q8NGB66 }\end{array}$ & $\begin{array}{l}\text { ZNF627 } \\
\text { OR4M2 }\end{array}$ & & & 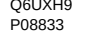 & $\begin{array}{l}\text { PAMR1 } \\
\text { IGFBP1 }\end{array}$ \\
\hline $\begin{array}{l}\text { Q99GZX3 } \\
\text { P88748 }\end{array}$ & $\begin{array}{l}\text { CHST6 } \\
\text { IGLV3-21 }\end{array}$ & & & $\begin{array}{l}\text { O754311 } \\
\text { O6UDP66 }\end{array}$ & $\begin{array}{l}\text { MTX2 } \\
\text { SPINT4 }\end{array}$ \\
\hline Q6ZTWO & TPGS1 & & & Q5HYN5 & $\begin{array}{l}\text { CT45A1 } \\
\text { Cons }\end{array}$ \\
\hline Q8TB05 & UBALD1 & & & Q5TOJ3 & C1ort220 \\
\hline $\begin{array}{l}0759947 \\
075366\end{array}$ & АTP5PD & & & $\begin{array}{l}\text { Q96668 } \\
\text { Q9UHC3 }\end{array}$ & $\begin{array}{l}\text { SETDD2 } \\
\text { ASIC3 }\end{array}$ \\
\hline P32754 & HPD & & & Q965Z5 & ADO \\
\hline & & & & $\begin{array}{l}\text { Q8NHUO } \\
\text { P293230 }\end{array}$ & СТ 45 АЗ \\
\hline & & & & & \\
\hline
\end{tabular}

Table_S8 statistically relevant interraction between Mlinc and proteins 


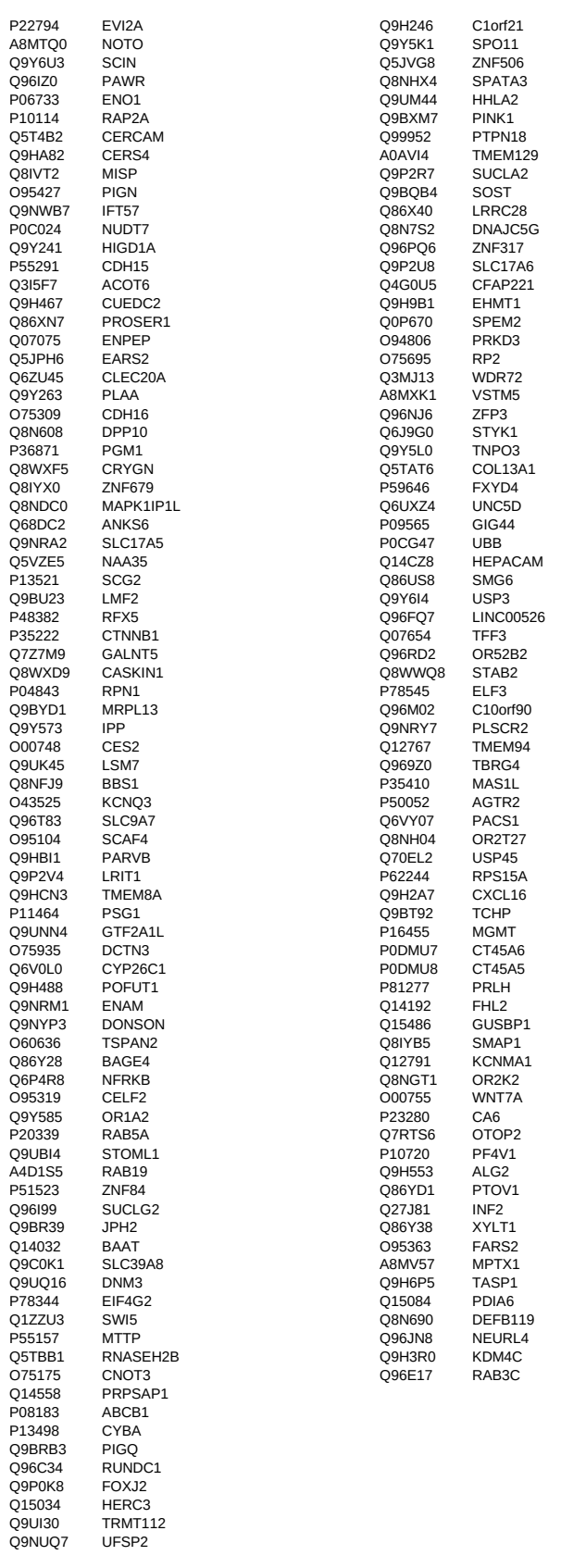

Table_S8 statistically relevant interraction between Mlinc and proteins

SUCLA2
SOST

政

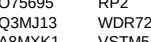

COL13A

$\begin{array}{ll}\text { POCG47 } & \text { UBB } \\ \text { Q14CZ8 } & \text { HEPACAM }\end{array}$

$\begin{array}{ll}\text { Q96FQ7 } & \text { USP3 } \\ \text { LINC00526 }\end{array}$

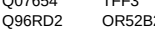

Q8WWQ1 STAB2

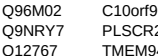

\begin{tabular}{ll} 
Q96920 & TBRG4 \\
P35410 & MAS1L \\
\hline
\end{tabular}

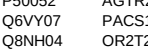

\begin{tabular}{ll} 
PODMU7 & CT45A6 \\
PODMU8 & CT45A5 \\
\hline
\end{tabular}

Q14192 FHI2

\begin{tabular}{ll} 
Q81YB5 & SMAP1 \\
Q12791 & KCNMA \\
\hline & 00755
\end{tabular}

$\begin{array}{ll}\text { Q8NGT1 } & \text { OR2K2 } \\ \text { O00755 } & \text { WNT7A }\end{array}$

$\begin{array}{ll}\text { P23280 } & \text { CA6 } \\ \text { Q7RTS6 } & \text { OTOP2 } \\ \text { PTSES }\end{array}$

$\begin{array}{ll}\text { Q9H553 } & \text { ALG2 } \\ \text { Q86YD1 } & \text { PTOV1 } \\ \text { P26Y1 } & \text { PF2 }\end{array}$

Q15084 PDIA6

$\begin{array}{ll}\text { Q963N8 } & \text { NEURL4 } \\ \text { Q9H3R0 } & \text { KDM4C } \\ \text { Q96517 } & \text { RAB3C }\end{array}$ 


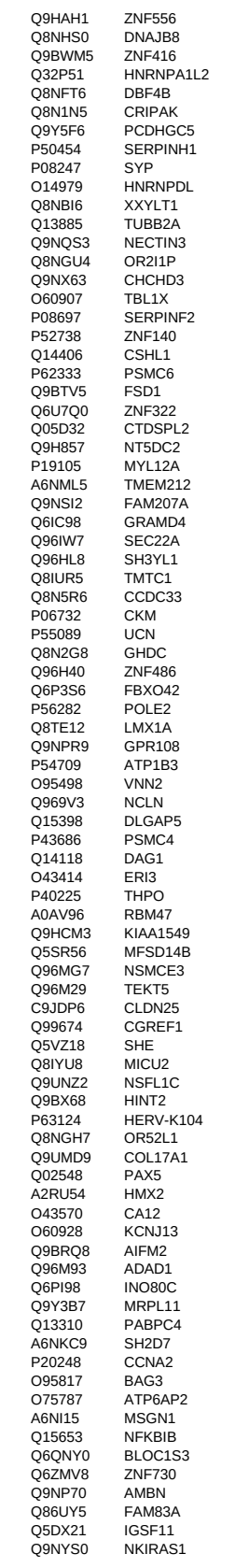

Table_s8 statistically relevant interraction between Mlinc and proteins 


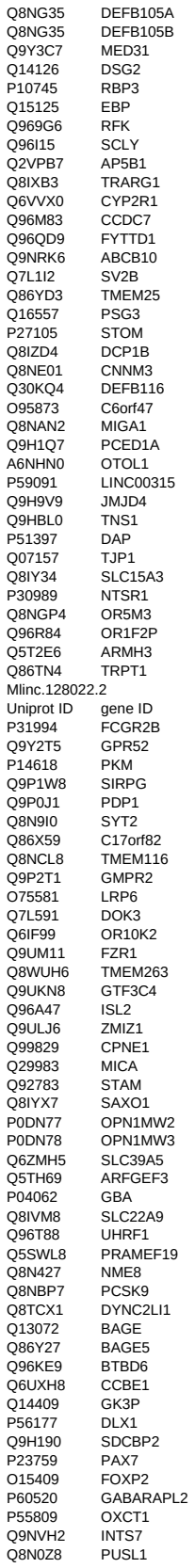




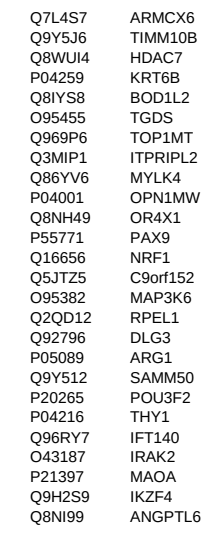

